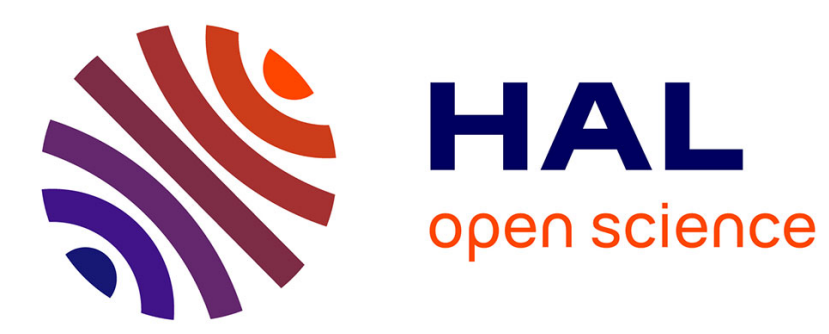

\title{
Statistical Inference via Convex Optimization
}

Anatoli B. Juditsky, Arkadi Nemirovski

\section{To cite this version:}

Anatoli B. Juditsky, Arkadi Nemirovski. Statistical Inference via Convex Optimization. 3rd cycle. France. 2018. cel-02047265

\section{HAL Id: cel-02047265 https://hal.science/cel-02047265}

Submitted on 24 Feb 2019

HAL is a multi-disciplinary open access archive for the deposit and dissemination of scientific research documents, whether they are published or not. The documents may come from teaching and research institutions in France or abroad, or from public or private research centers.
L'archive ouverte pluridisciplinaire HAL, est destinée au dépôt et à la diffusion de documents scientifiques de niveau recherche, publiés ou non, émanant des établissements d'enseignement et de recherche français ou étrangers, des laboratoires publics ou privés. 


\section{Statistical Inference via Convex Optimization}

Anatoli Juditsky and Arkadi Nemirovski

https://www.isye.gatech.edu/ nemirovs/StatOpt_LN.pdf

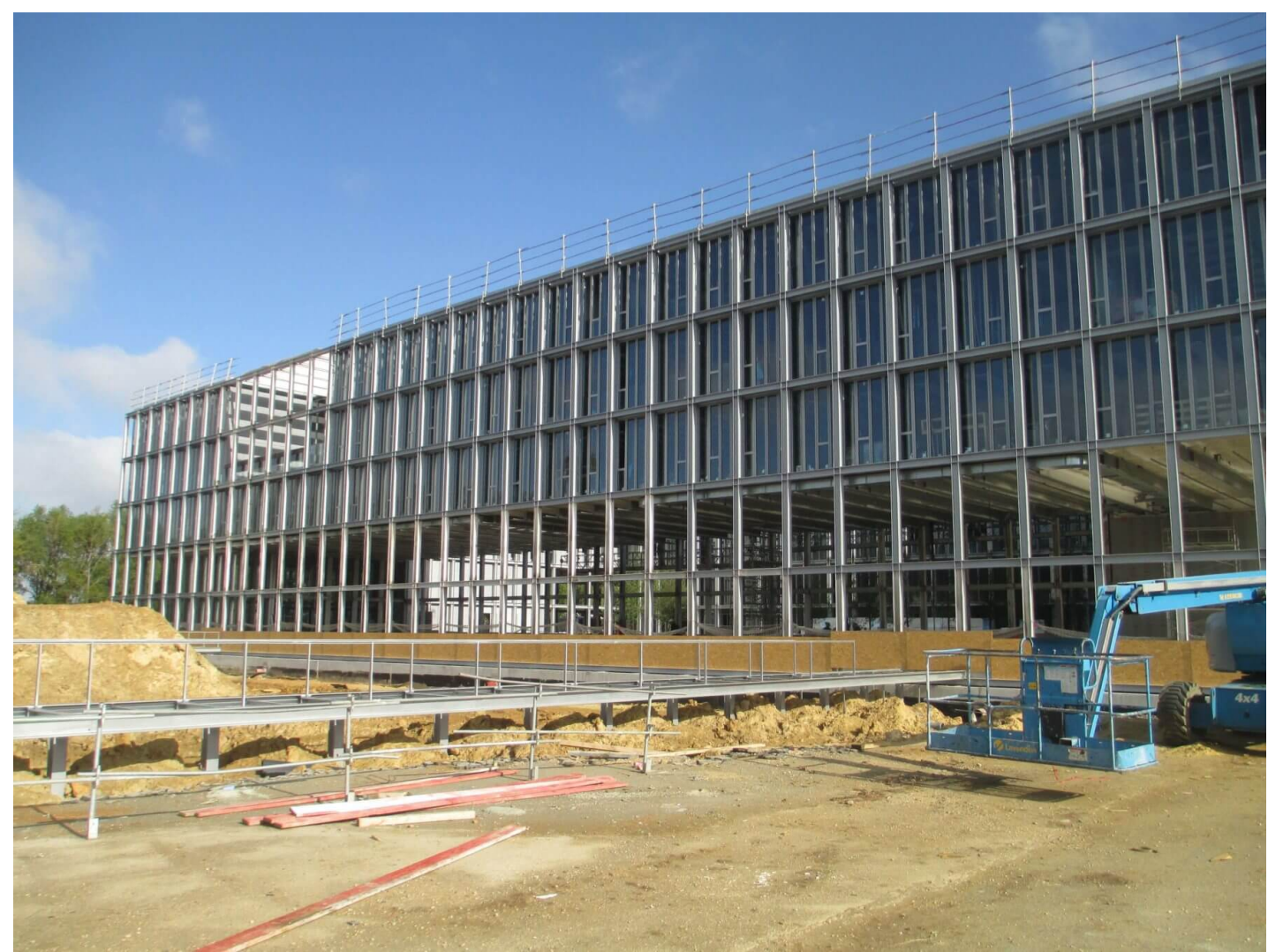

ENSAE ParisTech, October-November 2018

Transparencies in Dropbox https : //bit . Iy/2AhVMNH 


\section{Preface}

- Many inference procedures in Statistics reduce to optimization Example: MLE - Maximum Likelihood Estimation

Problem: Given a parametric family $\left\{p_{\theta}(\cdot): \theta \in \Theta\right\}$ of probability densities on $\mathbb{R}^{d}$ and a random observation $\omega$ drawn from some density $p_{\theta_{\star}}(\cdot)$ from the family, estimate the parameter $\theta_{\star}$.

MLE: Given $\omega$, maximize $p_{\theta}(\omega)$ over $\theta \in \Theta$ and use the maximizer $\widehat{\theta}=\widehat{\theta}(\omega)$ as an estimate of $\theta_{\star}$.

- In MLE, (most of the time) optimization is used for number crunching only and has nothing to do with motivation and performance analysis of MLE.

- Most of traditional applications of Optimization in Statistics are of "number crunching" nature. Though important, "number crunching" applications are beyond our scope 
Our scope: inference routines motivated and justified by Optimization Theory Our "working horse" will be Convex Optimization Theory - Convex Analysis, Optimality Conditions, Duality...

This choice is motivated by

- nice geometry of convex sets, functions, and optimization problems

- computational tractability of convex optimization implying computational efficiency of statistical inferences stemming from Convex Optimization. 


\section{Course validation}

Date: return your copy by Thursday, November 29

Options: Any choice of two (simple) or one (more involved, marked with ${ }^{\dagger}$ ) exercises from Chapters 2, 3, or 4 of the manuscript

https://www2.isye.gatech.edu// nemirovs/StatOpt_LN.pdf 


\section{FOR INTRODUCTION: \\ LINEAR FUNCTIONAL ESTIMATION IN WHITE NOISE}

- Donoho's Theorem

- Estimating Linear Functionals on Unions of Convex Sets 


\section{Linear Functional Estimation in White Noise Model}

We consider the model

$$
\omega=A x+\sigma \xi
$$

with

- observable $\omega \in \mathbb{R}^{d}$;

- unknown parameter $x \in \mathbb{R}^{n}$; it is known a priori that $x \in X, X \subset \mathbb{R}^{n}$ being convex and compact;

- sensing matrix $A \in \mathbb{R}^{d \times n}$;

- observation noise $\xi \sim N\left(0, I_{d}\right), \sigma>0$ is known.

Our goal is to estimate the value $g(x)$ at $x$ of a given linear functional

$$
g(z)=g^{T} z .
$$


Estimation risk: given $\epsilon \in(0,1)$, we define the risk of an estimator $\widehat{g}(\omega)$ of $g^{T} x$ on $X$ as the maximal width of confidence interval of level $1-\epsilon$ :

$$
\operatorname{Risk}_{\epsilon}[\widehat{g} \mid X]=\min \left\{\rho: \operatorname{Prob}_{x}\left\{\left|\widehat{g}(\omega)-g^{T} x\right|>\rho\right\} \leq \epsilon \forall x \in X\right\}
$$

We are specifically interested in the minmax risk

$$
\operatorname{RiskOpt}_{\epsilon}=\inf _{\widehat{g}} \operatorname{Risk}_{\epsilon}[\hat{g} \mid X],
$$

- We say that an estimator $\widehat{g}=\widehat{g}_{c, \phi}$ is affine if it is of the form

$$
\widehat{g}_{c, \phi}(\omega)=\phi^{T} \omega+c, \phi \in \mathbb{R}^{d}, c \in \mathbb{R} .
$$

We want to compare the minmax risk RiskOpt to $_{\epsilon}$ the maximal on $X$ risk of the best affine estimator:

$$
\operatorname{RiskA}_{\epsilon}=\inf _{c, \phi} \operatorname{Risk}_{\epsilon}\left[\widehat{g}_{c, \phi} \mid X\right] .
$$




\section{White Noise Model : Donoho's Theorem}

Thorem [Donoho, 1995] In the white noise model

$$
\text { RiskA }_{\epsilon} \leq C \text { RiskOpt }_{\epsilon}
$$

with $C \leq 1.25$ when $\epsilon \leq 0.01$.

Let us prove the following version of this result:

Proposition Let $\epsilon \in(0,1 / 2)$. There is an affine estimator $\widehat{g}_{c, \phi}(\cdot)$ of $g(x)$ such that

$$
\operatorname{Risk}_{\epsilon}\left[\widehat{g}_{c, \phi} \mid X\right] \leq \frac{q_{\mathcal{N}}(1-\epsilon / 2)}{q_{\mathcal{N}}(1-\epsilon)} \text { RiskOpt }_{\epsilon}
$$

where $q_{\mathcal{N}}(\alpha)$ is the $\alpha$-quantile of $\mathcal{N}(0,1)$.

Note: the ratio $\frac{q_{\mathcal{N}}(1-\epsilon / 2)}{q_{\mathcal{N}}(1-\epsilon)} \rightarrow 1$ as $\epsilon \downarrow+0$ 
Proof. $1^{\circ}$ Note that $\forall u \in X$,

$$
\begin{aligned}
\widehat{g}_{c, \phi}(\omega)-g(u) & =\phi^{T} \omega+c-g^{T} u=\phi^{T} A u-g^{T} u+\underbrace{\sigma \phi^{T} \xi}_{\sim \mathcal{N}\left(0, \sigma^{2}\|\phi\|_{2}^{2}\right)} \\
& \leq\left(A^{T} \phi-g\right) u+c+q_{\mathcal{N}}(1-\epsilon / 2) \sigma\|\phi\|_{2}
\end{aligned}
$$

with probability $1-\epsilon / 2$.

Thus, for $\rho=\max _{u \in X}\left(A^{T} \phi-g\right) u+c+q_{\mathcal{N}}(1-\epsilon / 2) \sigma\|\phi\|_{2}$,

$$
\sup _{u \in X} \operatorname{Prob}_{u}\{\widehat{g}(\omega)-g(u) \geq \rho\} \leq \epsilon / 2 \text {. }
$$

For the same reasons,

$$
\sup _{v \in X} \operatorname{Prob}_{v}\left\{\widehat{g}(\omega)-g(v) \leq-\rho^{\prime}\right\} \leq \epsilon / 2 .
$$

where $\rho^{\prime}=\max _{v \in X}\left(g-A^{T} \phi\right) v-c+q_{\mathcal{N}}(1-\epsilon / 2) \sigma\|\phi\|_{2}$.

Let us put

$$
c=\frac{1}{2}\left[\max _{u \in X}\left(g-A^{T} \phi\right)^{T} x-\max _{v \in X}\left(A^{T} \phi-g\right)^{T} v\right] .
$$

With this choice of $c$ we have $\rho=\rho^{\prime}$, and

$$
\rho=\frac{1}{2} \max _{u, v \in X}\left\{\Phi(\phi ; u, v):=\left[g^{T}(v-u)+\phi^{T} A(u-v)\right]+2 q_{\mathcal{N}}(1-\epsilon / 2) \sigma\|\phi\|_{2}\right\} .
$$

is the half-length of the confidence interval of $\widehat{g}(Y)$. We conclude that

$$
\operatorname{Risk}_{\epsilon}\left[\widehat{g}_{c, \phi} \mid X\right] \leq \operatorname{Opt}_{X}:=\min _{\phi}\left[\max _{u, v \in X} \Phi(\phi ; u, v)\right]
$$


$2^{\circ}$ Observe that $\Phi(\phi ; u, v)$ is convex and coercive in $\phi$ and concave in $u, v$. Thus, by the Sion-Kakutani theorem,

$$
\min _{\phi}\left[\max _{u, v \in X} \Phi(\phi ; u, v)\right]=\max _{u, v \in X}\left[\min _{\phi} \Phi(\phi ; u, v)\right]
$$

Note that

$$
\begin{aligned}
\min _{\phi} \Phi(\phi ; u, v) & =g^{T}(v-u)+\min _{\phi}\left[\phi^{T} A(u-v)+2 q_{\mathcal{N}}(1-\epsilon / 2) \sigma\left\|_{\phi}\right\|_{2}\right] \\
& =\left\{\begin{aligned}
g^{T}(v-u), & \|A(u-v)\|_{2} \leq 2 q_{\mathcal{N}}(1-\epsilon / 2) \sigma \\
-\infty, & \text { otherwise, }
\end{aligned}\right.
\end{aligned}
$$

and we conclude that

$$
\operatorname{Opt}_{X}=\max \left\{\frac{1}{2} g^{T}(v-u): u, v \in X,\|A(v-u)\|_{2} \leq 2 q_{\mathcal{N}}(1-\epsilon / 2) \sigma\right\} .
$$

$3^{\circ}$ We need to show that

$$
\operatorname{Opt}_{X} \leq \frac{q_{\mathcal{N}}(1-\epsilon / 2)}{q_{\mathcal{N}}(1-\epsilon)} \text { RiskOpt }_{\epsilon}:=\psi(\epsilon) \text { RiskOpt }_{\epsilon} .
$$

Let us assume, on the contrary, that

$$
\rho_{*}=\operatorname{RiskOpt}_{\epsilon}<\psi(\epsilon)^{-1} \mathrm{Opt}_{X} .
$$

Observe that $(*)$ is clearly solvable, let $[\bar{u} ; \bar{v}]$ be an optimal solution; we have

$$
2 \rho_{*}<\psi(\epsilon)^{-1} g^{T}(\bar{v}-\bar{u})<g^{T}(\bar{v}-\bar{u}) .
$$


Let now $[\widehat{u} ; \widehat{v}]$ be a "contraction" of $[\bar{u} ; \bar{v}]$ such that

$$
2 \rho_{*}=g^{T}(\widehat{v}-\widehat{u}) \text {. }
$$

We have

$$
\|A(\widehat{v}-\widehat{u})\|_{2}=\underbrace{\frac{\rho_{*}}{\mathrm{Opt}_{X}}}_{<\psi^{-1}}\|A(\bar{v}-\bar{u})\|_{2}<2 q_{\mathcal{N}}(1-\epsilon) \sigma .
$$

Now let us consider the problem of deciding between hypotheses

$$
H_{1}: x=\widehat{u} \text { and } H_{2}: x=\widehat{v} .
$$

Let $g_{*}(\cdot)$ be the (minmax) estimator of $g(u)$ with risk $\rho_{*}$.

Now consider the rule $\mathcal{T}(\cdot)$ deciding between $H_{0}$ and $H_{1}$ :

$$
\mathcal{T} \text { decides } H_{1} \text { if } g_{*}(\omega) \leq \frac{1}{2} g^{T}(\widehat{v}+\widehat{u}) \text {, and decides } H_{2} \text { otherwise. }
$$

By construction, the probability that $\mathcal{T}(\omega)$ rejects $H_{i}, i=1,2$ when it is true is $\leq \epsilon$. On the other hand,

$$
\|A \widehat{v}-A \widehat{u}\|_{2}<2 q_{\mathcal{N}}(1-\epsilon) \sigma .
$$

Then, by the Neyman-Pearson lemma, there is no test for $H_{1}$ and $H_{1}$ with the risk $2 \epsilon$, and we arrive at a contradiction. 


\section{Interpreting the result}

In order to find $\widehat{g}$ one has to solve a conic convex problem

$$
\text { Opt }_{X}=\max _{u, v \in X}\{\frac{1}{2} g^{T}(v-u): \underbrace{\|A(v-u)\|_{2}-2 q_{\mathcal{N}}(1-\epsilon / 2) \sigma}_{:=f(u, v)} \leq 0\} .
$$

It is a "well structured" problem and can be solved efficiently if $X$ is computationally tractable.

Note that an optimal solution $(\bar{u}, \bar{v})$ to this problem can be augmented by the Lagrange multiplier $\nu \geq 0$ such that the vectors

$$
\begin{aligned}
& e_{u}=\left.\frac{\partial}{\partial u}\right|_{(u, v)=(\bar{u}, \bar{v})}\left[\frac{1}{2} g^{T} u+\nu f(u, v)\right] \\
& e_{v}=\left.\frac{\partial}{\partial v}\right|_{(u, v)=(\bar{u}, \bar{v})}\left[-\frac{1}{2} g^{T} v+\nu f(u, v)\right]
\end{aligned}
$$

belong to the normal cone of $X \times X$ at $(\bar{u}, \bar{v})$ :

$$
\forall(u, v \in X): e_{u}^{T}(u-\bar{u}) \geq 0, e_{v}^{T}(v-\bar{v}) \geq 0 .
$$


There are two possible cases:

- $\nu=0$ : in this case the constraint $\|A(v-u)\|_{2} \leq 2 q_{\mathcal{N}}(1-\epsilon / 2)$ is not active, and

$$
[\bar{u} ; \bar{v}] \in \operatorname{Argmax}\left\{g^{T}(v-u): u, v \in X\right\} .
$$

We have $\phi_{*}=0$ and $c_{*}=g^{T}(\bar{u}+\bar{v})$;

- $\nu>0$ : in this case $\|A(\bar{v}-\bar{u})\|_{2}=2 q_{\mathcal{N}}(1-\epsilon / 2) \sigma$, and we set

$$
\widehat{g}(\omega)=\phi_{*}^{T} \omega+c_{*}, \quad \phi_{*}=\nu \frac{A(\bar{v}-\bar{u})}{\|A(\bar{u}-\bar{v})\|_{2}}, \quad c_{*}=\frac{1}{2} g^{T}(\bar{u}+\bar{v}) .
$$

One verifies straightforwardly that $\Phi\left(\phi_{*} ; u, v\right)$ attains its maximum over $u, v \in X$ at the point $(\bar{u}, \bar{v})$, and the maximal value is exactly $20 \mathrm{pt}{ }_{X}$. 


\section{Linear Functional Estimation over Unions of Convex Sets}

- Given

- collection $X_{j}, j=1, \ldots, J$, of nonempty convex compact sets in $\mathbb{R}^{n}$

- $d \times n$ matrix $A$

- linear function $g^{T} x$ on $\mathbb{R}^{n}$

- observation $\omega=A x+\xi, \xi \sim \mathcal{N}\left(0, \sigma^{2} I_{d}\right), \sigma>0$,

we want to estimate $g^{T} x$.

Same as before, we quantify the performance of an estimate $\widehat{g}(\cdot)$ by its $\epsilon$-risk:

$$
\operatorname{Risk}_{\epsilon}[\widehat{g} \mid X]=\min \left\{\rho: \operatorname{Prob}_{x}\left\{\left|\widehat{g}(\omega)-g^{T} x\right|>\rho\right\} \leq \epsilon \forall\left(j \leq J, x \in X_{j}\right)\right\} .
$$

We consider estimates which are aggregates of "pairwise" estimates of the form

$$
g_{i j}(\omega)=\phi_{i j}^{T} \omega+c_{i j}, \quad 1 \leq i, j \leq J .
$$


Let $\varepsilon \in(0,1), g(x)=\phi^{T} \omega+c, \phi \in \mathbb{R}^{d}, c \in \mathbb{R}$. Observe that $\forall x \in X$,

$$
\begin{aligned}
& g(\omega)-g^{T} x=\left(A^{T} \phi-g\right) x+c+\sigma \phi^{T} \xi \leq\left(A^{T} \phi-g\right) x+c+\sigma\|\phi\|_{2} q_{\mathcal{N}}(1-\varepsilon / 2), \\
& g^{T} x-g(\omega)=\left(g-A^{T} \phi\right) x-c+\sigma \phi^{T} \xi \leq\left(g-A^{T} \phi\right) x-c+\sigma\|\phi\|_{2} q_{\mathcal{N}}(1-\varepsilon / 2)
\end{aligned}
$$

with probability $1-\varepsilon / 2$. Therefore, for any $1 \leq i, j \leq J$,

$$
\begin{aligned}
& \forall u \in X_{i}: \quad g(\omega)-g^{T} u \leq \max _{u \in X_{i}}\left(A^{T} \phi-g\right) u+c+\sigma\|\phi\|_{2} q_{\mathcal{N}}(1-\varepsilon / 2) \\
& \forall v \in X_{j}: \quad g^{T} v-g(\omega) \leq \max _{v \in X_{j}}\left(g-A^{T} \phi\right) v-c+\sigma\|\phi\|_{2} q_{\mathcal{N}}(1-\varepsilon / 2) .
\end{aligned}
$$

Let

We conclude that $g(\omega)$ satisfies:

$$
c=\frac{1}{2}\left[\max _{v \in X_{j}}\left(g-A^{T} \phi\right) v-\max _{u \in X_{i}}\left(A^{T} \phi-g\right) u\right] .
$$

$$
\begin{aligned}
& \sup _{u \in X_{i}} \operatorname{Prob}_{u}\left\{g(\omega)-g^{T} u \geq \rho\right\} \leq \varepsilon / 2, \\
& \sup _{v \in X_{j}} \operatorname{Prob}_{v}\left\{g^{T} v-g(\omega) \geq \rho\right\} \leq \varepsilon / 2,
\end{aligned}
$$

where

$$
\rho=\frac{1}{2} \max _{u \in \boldsymbol{X}_{i}, v \in \boldsymbol{X}_{j}}\left\{\Phi(\phi ; u, v):=\left[g^{T}(v-u)+\phi^{T} A(u-v)\right]+2 q_{\mathcal{N}}(1-\varepsilon / 2) \sigma\|\phi\|_{2}\right\} .
$$


Building the estimate: Let $\varepsilon=\epsilon / J$. For $1 \leq i, j \leq J$, consider convex optimization problems

$$
\begin{aligned}
\text { Opt }_{i j} & =\min _{\phi}\left\{\max _{u \in X_{i}, v \in X_{j}} \frac{1}{2} \Phi(\phi ; u, v)\right\} \\
& =\min _{\phi}\left\{\max _{u \in X_{i}, v \in X_{j}} \frac{1}{2}\left[\left(g^{T}(v-u)+\phi^{T} A(u-v)\right)+2 q_{\mathcal{N}}\left(1-\frac{\epsilon}{2 J}\right) \sigma\|\phi\|_{2}\right]\right\} .
\end{aligned}
$$

Let $\phi_{i j}$ be feasible solutions to these problems, and $\rho_{i j}$ be the corresponding values of the objectives (the closer to Opt $\mathrm{O}_{i j}$, the better).

- We set

$$
g_{i j}(\omega)=\phi_{i j}^{T} \omega+c_{i j}, \quad c_{i j}=\frac{1}{2}\left[\max _{v \in X_{j}}\left(g-A^{T} \phi\right) v-\max _{u \in X_{i}}\left(A^{T} \phi-g\right) u\right] .
$$

thus ensuring

$$
\begin{aligned}
& \operatorname{Prob}_{u}\left\{g_{i j}(\omega)>g^{T} u+\rho_{i j}\right\} \leq \frac{\epsilon}{2 J}, \quad u \in X_{i} \\
& \operatorname{Prob}_{v}\left\{g_{i j}(\omega)<g^{T} v-\rho_{i j}\right\} \leq \frac{\epsilon}{2 J}, \quad v \in X_{j}
\end{aligned}
$$




$$
\begin{aligned}
& \operatorname{Prob}_{u}\left\{g_{i j}(\omega)>g^{T} u+\rho_{i j}\right\} \leq \frac{\epsilon}{2 J}, \quad u \in X_{i} \\
& \operatorname{Prob}_{v}\left\{g_{i j}(\omega)<g^{T} v-\rho_{i j}\right\} \leq \frac{\epsilon}{2 J}, \quad v \in X_{j}
\end{aligned}
$$

- Given observation $\omega$, we build the matrix

$$
\left[E_{i j}:=g_{i j}(\omega)\right]_{1 \leq i, j \leq J}
$$

and set

$$
\begin{aligned}
& r_{i}=\max _{j} E_{i j}, \quad r=\min _{i} r_{i}=\min _{i} \max _{j} E_{i j}, \\
& s_{j}=\min _{i} E_{i j}, \quad s=\max _{j} s_{j}=\max _{j} \min _{i} E_{i j} .
\end{aligned}
$$

The estimate $\widehat{g}(\omega)$ of $g^{T} x, x \in X=\cup_{j} X_{j}$, is set to

$$
\widehat{g}(\omega)=\frac{1}{2}[r+s] .
$$

Theorem $\epsilon$-Risk of the estimate $\hat{g}$ satisfies

$$
\operatorname{Risk}_{\epsilon}[\widehat{g} \mid X] \leq \rho:=\max _{i, j} \rho_{i j}
$$


Proof. $\mathbf{1}^{0}$. By $(b)$,

$$
\begin{aligned}
& \operatorname{Prob}_{u}\left\{g_{i j}(\omega)>g^{T} u+\rho_{i j}\right\} \leq \frac{\epsilon}{2 J}, u \in X_{i} \\
& \operatorname{Prob}_{v}\left\{g_{i j}(\omega)<g^{T} y-\rho_{i j}\right\} \leq \frac{\epsilon}{2 J}, v \in X_{j} \\
& E_{i j}=g_{i j}(\omega) \\
& r_{i}=\max _{j} E_{i j}, \quad r=\min _{i} r_{i}=\min _{i} \max _{j} E_{i j}, \\
& s_{j}=\min _{i} E_{i j}, \quad s=\max _{j} s_{j}=\max _{j} \min _{i} E_{i j} \\
& \widehat{g}\left(\omega^{K}\right)=\frac{1}{2}[r+s]
\end{aligned}
$$

$$
r_{i} \geq E_{i j} \geq s_{j} \Rightarrow r_{i} \geq s_{j} \forall i, j
$$

$\mathbf{2}^{0}$. Let $x \in X_{\ell}$, and let $\mathcal{E}$ be the event

$$
g_{\ell j}(\omega) \leq g^{T} u+\rho_{\ell j} \forall j \leq J, \text { and } g_{i \ell}(\omega) \geq g^{T} v-\rho \forall i \leq J,
$$

so that by $(a)$ and union bound,

$$
P(\mathcal{E}) \geq 1-\epsilon .
$$

Let $\Delta=\left[g^{T} x-\rho, g^{T} x+\rho\right]$. Note that when $\omega \in \mathcal{E}$, one has $r, c \in \Delta$.

Indeed, when $\omega \in \mathcal{E}$, we have

$$
r_{\ell j} \leq g^{T} x+\rho_{\ell j} \forall j \Rightarrow r_{\ell} \leq g^{T} x+\rho \Rightarrow r \leq g^{T} x+\rho,
$$

same as

$$
s_{i \ell} \geq g^{T} x-\rho_{i \ell} \forall i \Rightarrow s_{\ell} \geq g^{T} x-\rho \Rightarrow s \geq g^{T} x-\rho .
$$

Since $r \geq s$ by $(e)$, we conclude that $r, s \in \Delta$ whenever $\omega \in \mathcal{E}$. 


\section{Near-Optimality}

$$
\left.\operatorname{Opt}_{i j}=\min _{\phi}\{\max _{u \in X_{i}, v \in X_{j}} \frac{1}{2} \underbrace{\left[\left(g^{T}(v-u)+\phi^{T} A(u-v)\right)+2 q_{\mathcal{N}}\left(1-\frac{\epsilon}{2 J}\right) \sigma\|\phi\|_{2}\right.}_{\Phi\left(\phi_{i j} ; u, v\right)}]\right\}
$$

Because $\Phi\left(\phi_{i j} ; u, v\right)$ is convex and coercive in $\phi$ and concave in $u, v$ while $X_{i}, X_{j}$ are convex compact sets, by the Sion-Kakutani theorem,

$$
\min _{\phi}\left[\max _{u \in X_{i}, v \in X_{j}} \Phi\left(\phi_{i j} ; u, v\right)\right]=\max _{u \in X_{i}, v \in X_{j}}\left[\min _{\phi} \Phi(\phi ; u, v)\right]
$$

Same as above, we conclude that $O p t_{i j}$ is either $-\infty$, or

$$
\mathrm{Opt}_{i j}=\max \left\{\frac{1}{2} g^{T}(v-u): u \in X_{i}, v \in X_{j},\|A(v-u)\|_{2} \leq 2 q_{\mathcal{N}}\left(1-\frac{\epsilon}{2 J}\right) \sigma\right\} .
$$

It is immediately seen that $\mathrm{Opt}_{i i}$ is a nonnegative real, so that

$$
\text { Opt }:=\max _{i, j} \mathrm{Opt}_{i j} \geq 0 \text {. }
$$


Note that we can make the bound $\rho$ on $\epsilon$-risk of our estimate arbitrarily close to Opt by properly selecting corresponding feasible solutions.

We are to show that Opt is nearly the optimal $\epsilon$-risk in our estimation problem

$$
\operatorname{RiskOpt}_{\epsilon}=\inf _{\widehat{g}} \operatorname{Risk}_{\epsilon}[\hat{g} \mid X] .
$$

Theorem Let $\epsilon \in(0,1 / 2)$. Assume that we are in the special case where for every $i, j$ there exists $x_{i j} \in X_{i} \cap X_{j}$. Then

$$
\text { Opt } \leq \frac{q_{\mathcal{N}}\left(1-\frac{\epsilon}{2 J}\right)}{q_{\mathcal{N}}(1-\epsilon)} \text { RiskOpt }_{\epsilon} .
$$




$$
\mathrm{Opt}_{i j}=\max \left\{\frac{1}{2} g^{T}(v-u): u \in X_{i}, v \in X_{j},\|A(v-u)\|_{2} \leq 2 q_{\mathcal{N}}\left(1-\frac{\epsilon}{2 J}\right) \sigma\right\}
$$

Proof. $1^{o}$ We are to show that for all $1 \leq i, j \leq J$

$$
\mathrm{Opt}_{i j} \leq \underbrace{\frac{q_{\mathcal{N}}\left(1-\frac{\epsilon}{2 J}\right)}{q_{\mathcal{N}}(1-\epsilon)}}_{:=\psi(\epsilon)} \text { RiskOpt }_{\epsilon}[\geq 0]
$$

Let us associate with $(*)$ a parametric problem

$$
w_{i j}(s)=\max \left\{\frac{1}{2} g^{T}(v-u): u \in X_{i}, v \in X_{j},\|A(v-u)\|_{2} \leq s\right\} .
$$

Note that $\left(P_{s}\right)$ is convex (in the sense that its objective is concave and the domain is convex) and feasible $\forall s \geq 0$ (with a feasible solution $u=v=x_{i j}$ ) with $w_{i j}(0) \geq 0$. We conclude that $w_{i j}(s)$ is nonnegative, concave and bounded on $\mathbb{R}_{+} \Rightarrow w_{i j}(s)$ is continuous for $s>0$. 


$$
w_{i j}(s)=\max \left\{\frac{1}{2} g^{T}(v-u): u \in X_{i}, v \in X_{j},\|A(v-u)\|_{2} \leq s\right\} .
$$

We claim that

$$
w_{i j}\left(s_{*}\right) \leq \text { RiskOpt }_{\epsilon}, \quad s_{*}=2 \sigma q_{\mathcal{N}}(1-\epsilon) .
$$

Let us assume, on the contrary, that $w_{i j}\left(s_{*}\right)>\mathrm{RiskOpt}_{\epsilon}$, so that for some $s^{\prime} \in\left(0, s_{*}\right)$, $w_{i j}\left(s^{\prime}\right)>$ RiskOpt $_{\epsilon}$. Then there exist $\bar{u} \in X_{i}$ and $\bar{v} \in X_{j}$ which are feasible for $\left(P_{s^{\prime}}\right)$ and such that

$$
\|A(\bar{u}-\bar{v})\|_{2}<2 \sigma q_{\mathcal{N}}(1-\epsilon),
$$

and

$$
\frac{1}{2} g^{T}(\bar{v}-\bar{u})>\text { RiskOpt }_{\epsilon} .
$$

We have already seen that this is impossible by the Neyman-Pearson lemma, thus arriving to a contradiction.

$\mathbf{2}^{\circ}$. We conclude that $w_{i j}\left(s_{*}\right) \leq$ RiskOpt $_{\epsilon}$. Now, we for

$$
\bar{s}=\psi(\epsilon) s_{*}=2 q_{\mathcal{N}}\left(1-\frac{\epsilon}{2 J}\right) \sigma
$$

we have by concavity of $w_{i j}(s)$ and due to $w_{i j}(0) \geq 0$ :

$$
w_{i j}(\bar{s}) \leq \psi(\epsilon) w_{i j}\left(s^{\prime}\right) \leq \psi(\epsilon) \text { RiskOpt }_{\epsilon} .
$$




\section{Numerical Illustration}

Setup: Given $J=100$ points $x_{j} \in \mathbb{R}^{20}$ and stationary $K$-repeated observation noisy observation

$$
\omega^{K}=\left(\omega_{1}, . ., \omega_{K}\right), \omega_{k} \sim \mathcal{N}\left(A x, I_{20}\right)
$$

of one of the points (we do not know which one!), we want to recover the first entry of the point. - $A$ : randomly generated matrix

- $\epsilon=0.01$.

Note: We are in the situation where $X_{i}=\left\{x_{i}\right\}$ are singletons.

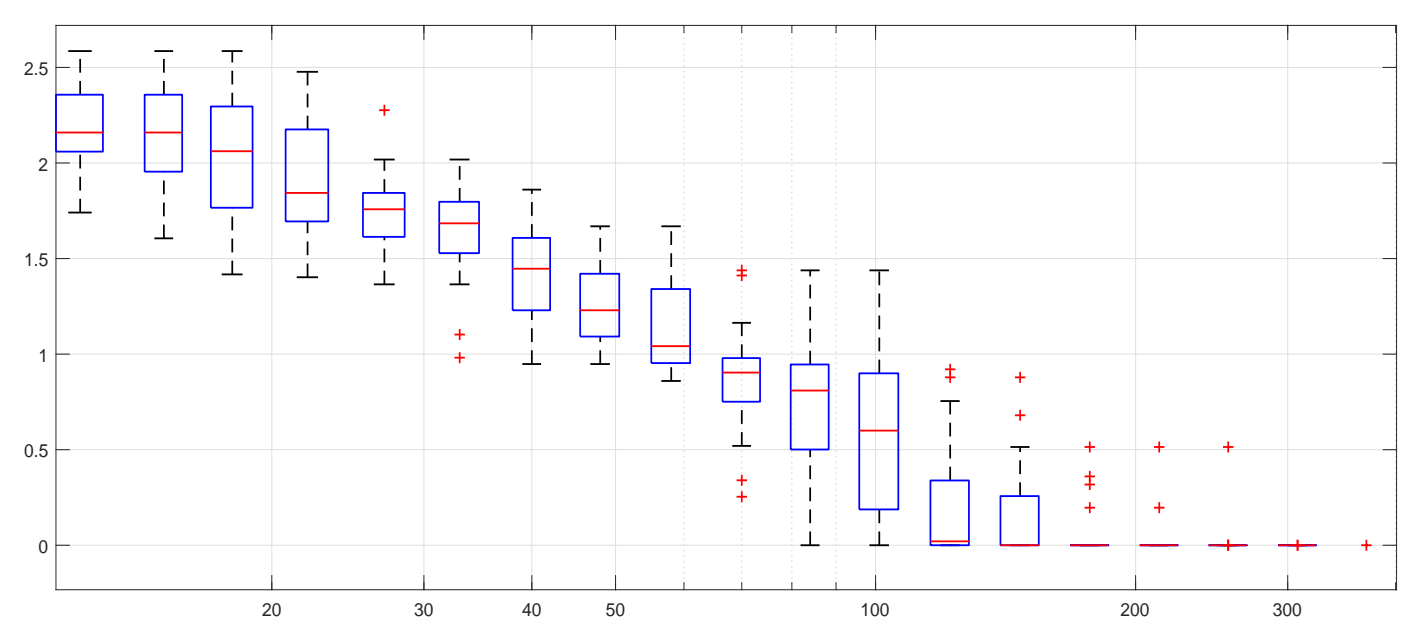

$\operatorname{Opt}(K)$ vs. $\sigma^{-2}$, data over 20 randomly generated collections $\left\{x_{i}\right\}_{i=1}^{100}$ 


\section{HYPOTHESIS TESTING, I}

- Preliminaries

- Tests \& Risks

- Repeated Observations

- 2-Point Lower Risk Bound

- Pairwise Tests via Euclidean Separation

- From Pairwise to Multiple Hypothesis Testing 


\section{Hypothesis Testing Problem}

\section{Given}

- observation space $\Omega$ where our observations take values,

- $L$ families $\mathcal{P}_{1}, \mathcal{P}_{2}, \ldots, \mathcal{P}_{L}$ of probability distributions on $\Omega$, and

- an observation $\omega$ - a realization of random variable with unknown probability distribution $P$ known to belong to one of the families $\mathcal{P}_{\ell}: P \in \bigcup_{\ell=1}^{L} \mathcal{P}_{\ell}$, we want to decide to which one of the families $\mathcal{P}_{\ell}$ the distribution $P$ belongs.

Equivalent wording: Given the outlined data, we want to decide on $L$ hypotheses $H_{1}, \ldots, H_{L}$, with $\ell$-th hypothesis $H_{\ell}$ stating that $P \in \mathcal{P}_{\ell}$.

A test is a function $\mathcal{T}(\cdot)$ on $\Omega$. The value $\mathcal{T}(\omega)$ of this function at a point $\omega \in \Omega$ is a subset of the set $\{1, \ldots, L\}$.

- relation $\ell \in \mathcal{T}(\omega)$ is interpreted as "given observation $\omega$, the test accepts the hypothesis $H_{\ell}$ "

- relation $\ell \notin \mathcal{T}(\omega)$ is interpreted as "given observation $\omega$, the test rejects the hypothesis $H_{\ell}$ " 


\section{Simple tests}

- $\mathcal{T}$ is called simple, if $\mathcal{T}(\omega)$ is a singleton for every $\omega \in \Omega$.

Risks of a simple test $\mathcal{T}$, are defined as follows:

- $\ell$-th partial risk of $\mathcal{T}$ is the (worst-case) probability to reject $\ell$-th hypothesis when it is true:

$$
\operatorname{Risk}_{\ell}\left[\mathcal{T} \mid H_{1}, \ldots, H_{L}\right]=\sup _{P \in \mathcal{P}_{\ell}} \operatorname{Prob}_{\omega \sim P}\{\ell \notin \mathcal{T}(\omega)\}
$$

- total risk of $\mathcal{T}$ is the sum of all partial risks:

$$
\operatorname{Risk}_{\text {tot }}\left[\mathcal{T} \mid H_{1}, \ldots, H_{L}\right]=\sum_{1 \leq \ell \leq L} \operatorname{Risk}_{\ell}\left[\mathcal{T} \mid H_{1}, \ldots, H_{L}\right] .
$$

- risk of $\mathcal{T}$ is the maximum of all partial risks:

$$
\operatorname{Risk}\left[\mathcal{T} \mid H_{1}, \ldots, H_{L}\right]=\max _{1 \leq \ell \leq L} \operatorname{Risk}_{\ell}\left[\mathcal{T} \mid H_{1}, \ldots, H_{L}\right] .
$$


Remark: What was called test is in fact a deterministic test.

A randomized test is a deterministic function $\mathcal{T}(\omega, \eta)$ of observation $\omega$ and independent of $\omega$ random variable $\eta \sim P_{\eta}$ with once for ever fixed distribution (e.g., $P_{\eta}=$ Uniform $[0,1]$ ).

- Values $\mathcal{T}(\omega, \eta)$ of $\mathcal{T}$ are subsets of $\{1, \ldots, L\}$ (singletons for a simple test).

- Given observation $\omega$, we "flip a coin" (draw a realization of $\eta$ ), accept hypotheses $H_{\ell}, \ell \in \mathcal{T}(\omega, \eta)$, and reject all other hypotheses.

- Partial risks of a randomized test are

$$
\operatorname{Risk}_{\ell}\left[\mathcal{T} \mid H_{1}, \ldots, H_{L}\right]=\sup _{P \in \mathcal{P}_{\ell}} \operatorname{Prob}_{(\omega, \eta) \sim P \times P_{\eta}}\{\ell \notin \mathcal{T}(\omega, \eta)\} .
$$

Exactly as above, these risks give rise to the total risk and risk of $\mathcal{T}$. 


\section{Testing from repeated observations}

There are situations where an inference can be based on several observations $\omega_{1}, \ldots, \omega_{K}$ rather than on a single observation. Our related setup is as follows:

We are given $L$ families $\mathcal{P}_{\ell}, \ell=1, \ldots, L$, of probability distributions on observation space $\Omega$ and a collection

$$
\omega^{K}=\left(\omega_{1}, \ldots, \omega_{K}\right),
$$

and want to make conclusions on how the distribution of $\omega^{K}$ "is positioned" w.r.t. the families $\mathcal{P}_{\ell}, 1 \leq \ell \leq L$.

Specifically, we are interested in three situations of this type. 


\section{A. Stationary $K$-repeated observations}

Situation: $\omega_{1}, \ldots, \omega_{K}$ are drawn independently of each other from the same distribution $P$. Our goal is to decide, given $\omega^{K}$, on the hypotheses $P \in \mathcal{P}_{\ell}$, $\ell=1, \ldots, L$.

Equivalently: Families $\mathcal{P}_{\ell}$ of probability distributions of $\omega \in \Omega, 1 \leq \ell \leq L$, give rise to the families

$$
\mathcal{P}_{\ell}^{K}=\{P^{K}=\underbrace{P \times \ldots \times P}_{K}: P \in \mathcal{P}_{\ell}\}
$$

of probability distributions on $\Omega^{K}=\underbrace{\Omega \times \ldots \times \Omega}_{K}$. Given observation $\omega^{K} \in$ $\Omega^{K}$, we want to decide on the hypotheses

$$
H_{\ell}^{K}: \omega^{K} \sim P^{K} \in \mathcal{P}_{\ell}^{K}, 1 \leq \ell \leq L .
$$




\section{B. Semi-stationary $K$-repeated observations}

Situation: "The nature" selects somehow a sequence $P_{1}, \ldots, P_{K}$ of distributions on $\Omega$, and then draws, independently across $k$, observations $\omega_{k}$ from these distributions:

$$
\omega_{k} \sim P_{k} \text { are independent across } k \leq K
$$

Our goal is to decide, given $\omega^{K}=\left(\omega_{1}, \ldots, \omega_{K}\right)$, on the hypotheses

$$
\left\{P_{k} \in \mathcal{P}_{\ell}, 1 \leq k \leq K\right\}, \ell=1, \ldots, L .
$$

Equivalently: Families $\mathcal{P}_{\ell}$ of probability distributions of $\omega \in \Omega, 1 \leq \ell \leq L$, give rise to the families

$$
\mathcal{P}_{\ell}^{\oplus, K}=\bigoplus_{k=1}^{K} \mathcal{P}_{\ell}:=\left\{P^{K}=P_{1} \times \ldots \times P_{K}: P_{k} \in \mathcal{P}_{\ell}, 1 \leq k \leq K\right\}
$$

of probability distributions on $\Omega^{K}=\underbrace{\Omega \times \ldots \times \Omega}_{K}$. Given observation $\omega^{K} \in$ $\Omega^{K}$, we want to decide on the hypotheses

$$
H_{\ell}^{\oplus, K}: \omega^{K} \sim P^{K} \in \mathcal{P}_{\ell}^{\oplus, K}, 1 \leq \ell \leq L .
$$




\section{Quasi-stationary $K$-repeated observations:}

We observe random sequence $\omega^{K}=\left(\omega_{1}, \ldots, \omega_{K}\right)$ generated as follows:

There exists a random sequence $\zeta_{1}, \ldots, \zeta_{K}$ of driving factors such that for $1 \leq k \leq K$

- $\omega_{k}$ is a deterministic function of $\zeta^{k}=\left(\zeta_{1}, \ldots, \zeta_{k}\right)$

- conditional, given $\zeta^{k-1}$, distribution of $\omega_{k}$ always belongs to $\mathcal{P}_{\ell}$.

Our goal is to decide, given $\omega^{K}$, on the underlying $\ell$.

Equivalently: Families $\mathcal{P}_{\ell}$ of probability distributions on $\Omega, 1 \leq \ell \leq L$, give rise to the quasi-direct products $\mathcal{P}_{\ell}^{\otimes, K}=\bigotimes_{k=1}^{K} \mathcal{P}_{\ell}$ of families $\mathcal{P}_{\ell}$.

Family $\bigotimes_{k=1}^{K} \mathcal{P}_{\ell}$ is comprised of all probability distributions on $\Omega^{K}=\underbrace{\Omega \times \ldots \times \Omega}_{K}$ which can be obtained from $\mathcal{P}_{\ell}$ via the above "driving factors" mechanism. Given observation $\omega^{K} \in \Omega^{K}$, we want to decide on the hypotheses

$$
H_{\ell}^{\otimes, K}: \omega^{K} \sim P^{K} \in \mathcal{P}_{\ell}^{\otimes, K}, 1 \leq \ell \leq L .
$$




\section{Important fact: 2-point lower risk bound}

Consider a simple pairwise test deciding on two simple hypotheses on the distribution $P$ of observation $\omega \in \Omega$ :

$$
H_{1}: P=P_{1}, \quad H_{2}: P=P_{2} .
$$

Let $P_{1}, P_{2}$ have densities $p_{1}, p_{2}$ w.r.t. some reference measure $\Pi$ on $\Omega$. Then the total risk of every test $\mathcal{T}$ deciding on $\mathrm{H}_{1}, \mathrm{H}_{2}$ admits lower bound as follows:

$$
\operatorname{Risk}_{\text {tot }}\left[\mathcal{T} \mid H_{1}, H_{2}\right] \geq \int_{\Omega} \min \left[p_{1}(\omega), p_{2}(\omega)\right] \Pi(d \omega) .
$$

As a result,

$$
\operatorname{Risk}\left[\mathcal{T} \mid H_{1}, H_{2}\right] \geq \frac{1}{2} \int_{\Omega} \min \left[p_{1}(\omega), p_{2}(\omega)\right] \Pi(d \omega) .
$$

The bound does not depend on the choice of $\Pi$ (for example, we can always take $\Pi=P_{1}+P_{2}$ ). 


$$
\operatorname{Risk}\left[\mathcal{T} \mid H_{1}, H_{2}\right] \geq \frac{1}{2} \int_{\Omega} \min \left[p_{1}(\omega), p_{2}(\omega)\right] \Pi(d \omega) .
$$

Proof (for deterministic test). Simple test deciding on $H_{1}, H_{2}$ must accept $H_{1}$ and reject $H_{2}$ on some subset $\Omega_{1}$ of $\Omega$ and must reject $H_{1}$ and accept $H_{2}$ on the complement $\Omega_{2}=\Omega \backslash \Omega_{1}$ of this set. We have

$$
\begin{aligned}
& \operatorname{Risk}_{1}\left[\mathcal{T} \mid H_{1}, H_{2}\right]=\iint_{\Omega_{2}} p_{1}(\omega) \Pi(d \omega) \geq \int \min \left[p_{1}(\omega), p_{2}(\omega)\right] \Pi(d \omega) \\
& \operatorname{Risk}_{2}\left[\mathcal{T} \mid H_{1}, H_{2}\right]=\int_{\Omega_{1}} p_{2}(\omega) \Pi(d \omega) \geq \int_{\Omega_{1}} \min \left[p_{1}(\omega), p_{2}(\omega)\right] \Pi(d \omega),
\end{aligned}
$$

thus

$$
\begin{aligned}
\operatorname{Risk}_{\text {tot }}\left[\mathcal{T} \mid H_{1}, H_{2}\right] & \geq \int_{\Omega_{2}} \min \left[p_{1}(\omega), p_{2}(\omega)\right] \Pi(d \omega)+\int_{\Omega_{1}} \min \left[p_{1}(\omega), p_{2}(\omega)\right] \Pi(d \omega) \\
& =\int_{\Omega} \min \left[p_{1}(\omega), p_{2}(\omega)\right] \Pi(d \omega)
\end{aligned}
$$


Remark: In the situation in question, consider the test $\mathcal{T}_{*}$ :

$\mathcal{T}_{*}$ accepts $H_{1}$ if $p_{1}(\omega) \geq p_{2}(\omega)$, and accepts $H_{2}$ if $p_{1}(\omega)<p_{2}(\omega)$

(Neyman-Pearson or Likelihood Ratio test).

Lemma [Neyman-Pearson] For any test $\mathcal{T}$

$$
\operatorname{Risk}_{\text {tot }}\left[\mathcal{T} \mid H_{1}, H_{2}\right] \geq \operatorname{Risk}_{\text {tot }}\left[\mathcal{T}_{*} \mid H_{1}, H_{2}\right]=\int_{\Omega} \min \left[p_{1}(\omega), p_{2}(\omega)\right] \Pi(d \omega) .
$$

Example: Gaussian distributions. Let $\Omega=\mathbb{R}^{d}$, our observation

$$
\omega=x+\xi,
$$

where $x$ is deterministic and $\xi \sim \mathcal{N}(0, I)$, i.e., has the density $p(u)=(2 \pi)^{-d / 2} e^{-\frac{1}{2} u^{T} u}$. Our goal is to decide on two simple hypotheses: $H_{1}: x=x_{1}$ and $H_{2}: x=x_{2}, x_{1}, x_{2}$ being two given points.

The corresponding test $\mathcal{T}_{*}$ decides $H_{1}$ if

$$
\phi_{*}(\omega)=e^{T}\left[\omega-\frac{1}{2}\left(x_{1}+x_{2}\right)\right] \geq 0, \quad e=\frac{x^{1}-x^{2}}{\left\|x^{1}-x^{2}\right\|_{2}}
$$

and decides $\mathrm{H}_{2}$ otherwise. Its total risk is given by

$$
\begin{aligned}
\text { Risk }_{\text {tot }}\left[\mathcal{T} \mid H_{1}, H_{2}\right]= & P_{1}\left(\phi_{*}(\omega)<0\right)+P_{2}\left(\phi_{*}(\omega) \geq 0\right) \\
= & 2 \operatorname{Prob}_{\zeta \sim N(0,1)}\left\{\zeta \geq \frac{1}{2}\left\|x_{2}-x_{1}\right\|_{2}\right\}=\operatorname{erfc}\left(\frac{\left\|x_{2}-x_{1}\right\|_{2}}{2 \sqrt{2}}\right) . \\
& -1.10-
\end{aligned}
$$




$$
\begin{gathered}
e=\frac{x^{1}-x^{2}}{\left\|x^{1}-x^{2}\right\|_{2}} \\
\phi(\omega)=e^{T} \omega-\underbrace{\frac{1}{2} e^{T}\left[x^{1}+x^{2}\right]} \\
\Pi=\left\{\omega:\left\|\omega-x^{1}\right\|_{2}=\left\|\omega-x^{2}\right\|_{2}\right\}=\{\omega: \phi(\omega)=0\}
\end{gathered}
$$

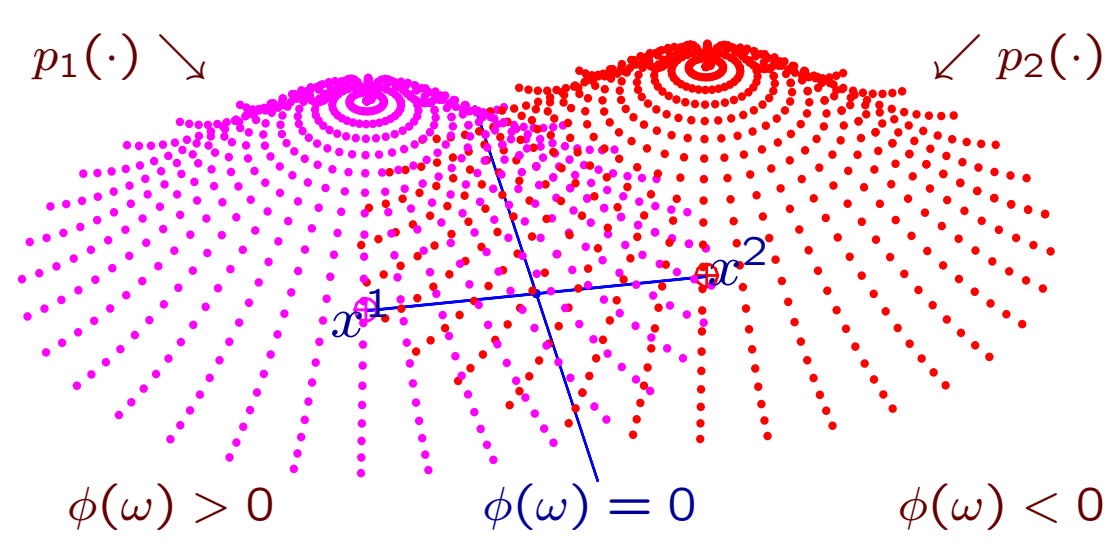


Corollary. Consider $L$ hypotheses $H_{\ell}: P \in \mathcal{P}_{\ell}, \ell=1,2, \ldots, L$, on the distribution $P$ of observation $\omega \in \Omega$, let $\ell \neq \ell^{\prime}$ and let $P_{\ell} \in \mathcal{P}_{\ell}, P_{\ell^{\prime}} \in \mathcal{P}_{\ell^{\prime}}$.

The risk of any simple test $\mathcal{T}$ deciding on $H_{1}, \ldots, H_{L}$ can be lower-bounded as

$$
\operatorname{Risk}\left[\mathcal{T} \mid H_{1}, \ldots, H_{L}\right] \geq \frac{1}{2} \int_{\Omega} \min \left[P_{\ell}(d \omega), P_{\ell^{\prime}}(d \omega)\right],
$$

where, by convention, the integral in the right hand side is

$$
\int_{\Omega} \min \left[p_{\ell}(\omega), p_{\ell^{\prime}}(\omega)\right] \Pi(d \omega),
$$

with $p_{\ell}, p_{\ell^{\prime}}$ being the densities of $P_{\ell}, P_{\ell^{\prime}}$ w.r.t. $\Pi=P_{\ell}+P_{\ell^{\prime}}$.

Indeed, risk of $\mathcal{T}$ cannot be less than the risk of the naturally induced by $\mathcal{T}$ simple test deciding on two simple hypotheses $P=P_{\ell}, P=P_{\ell^{\prime}}$, specifically, the simple test which, given observation $\omega$ accepts the hypothesis $P=P_{\ell}$ whenever $\ell \in \mathcal{T}(\omega)$ and accepts the hypothesis $P=P_{\ell^{\prime}}$ otherwise. 


\section{Extension: Euclidean separation}

Given observation $\omega=x+\xi$ with observation noise $\xi \sim \mathcal{N}(0, I)$, we want to decide on two composite hypotheses $H_{1}$, and $H_{2}$ :

$$
H_{1}: x \in X_{1}, \quad H_{2}: x \in X_{2} \text {, }
$$

where $X_{1}, X_{2}$ are nonempty nonintersecting, closed and convex sets, and one of the sets is bounded.

Elementary fact: With $X_{1}, X_{2}$ as above, consider the convex minimization problem

$$
\text { Opt }=\min _{x^{1} \in X_{1}, x^{2} \in X_{2}} \frac{1}{2}\left\|x^{1}-x^{2}\right\|_{2}
$$

The problem is solvable. Let $\left(x_{*}^{1}, x_{*}^{2}\right)$ be an optimal solution, and let

$$
\phi(\omega)=e^{T} \omega-c, \quad e=\frac{x_{*}^{1}-x_{*}^{2}}{\left\|x_{*}^{1}-x_{*}^{2}\right\|_{2}}, \quad c=\frac{1}{2} e^{T}\left[x_{*}^{1}+x_{*}^{2}\right]
$$

Then the stripe $\{\omega:-\mathrm{Opt} \leq \phi(\omega) \leq \mathrm{Opt}\}$ separates $X_{1}$ and $X_{2}$ :

$$
\phi\left(x^{1}\right) \geq \phi\left(x_{*}^{1}\right)=\text { Opt } \forall x^{1} \in X_{1}, \quad \phi\left(x^{2}\right) \leq \phi\left(x_{*}^{2}\right)=- \text { Opt } \forall x^{2} \in X_{2}
$$




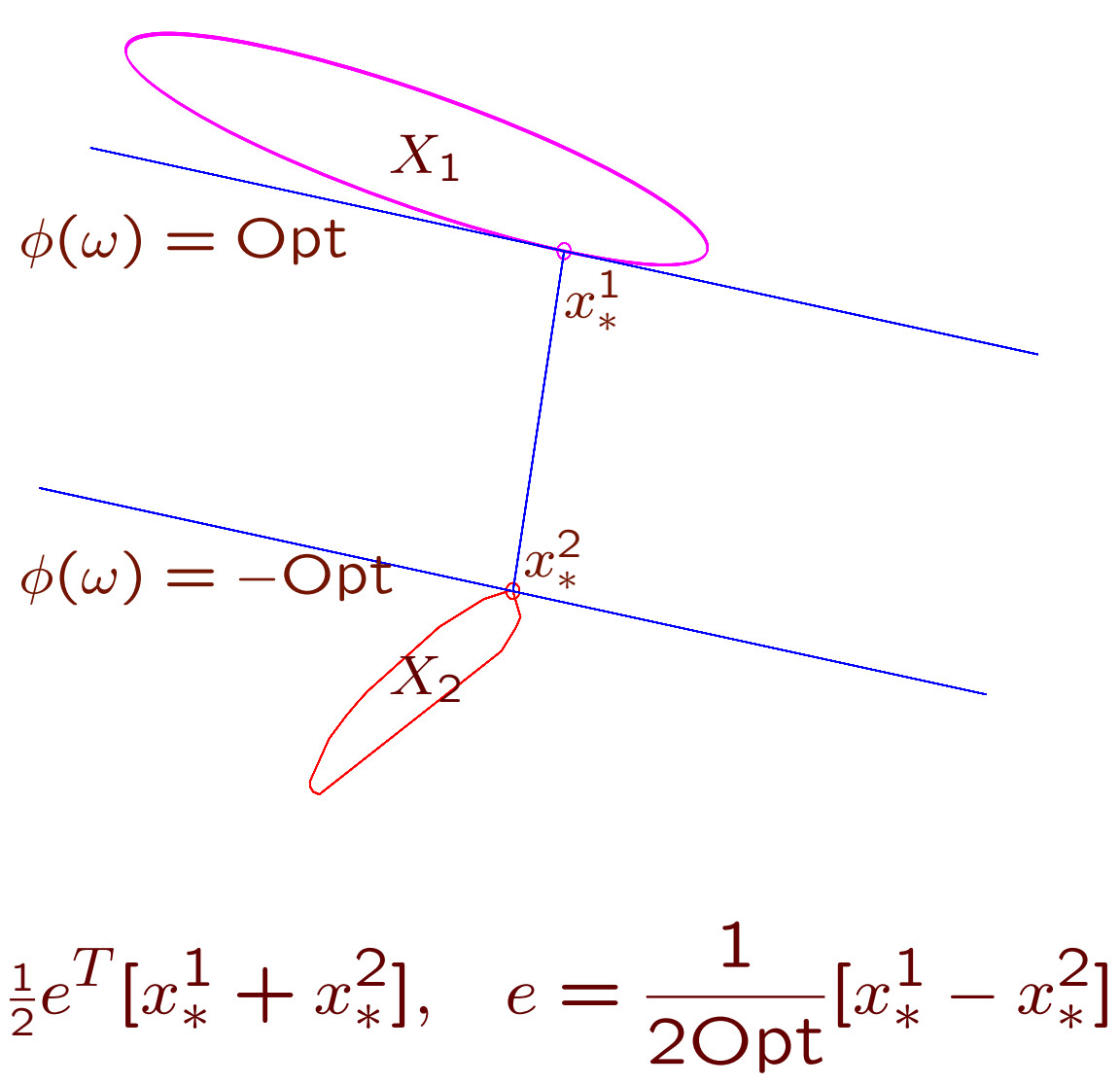

where $\left[x_{*}^{1}, x_{*}^{2}\right]$ is an optimal solution to convex optimization problem

$$
\text { Opt }=\min _{x^{1} \in X_{1}, x^{2} \in X_{2}} \frac{1}{2}\left\|x^{1}-x^{2}\right\|_{2}
$$

While optimal solution si not necessarily unique, $\phi(\cdot)$ is uniquely defined by $X_{1}, X_{2}$. 


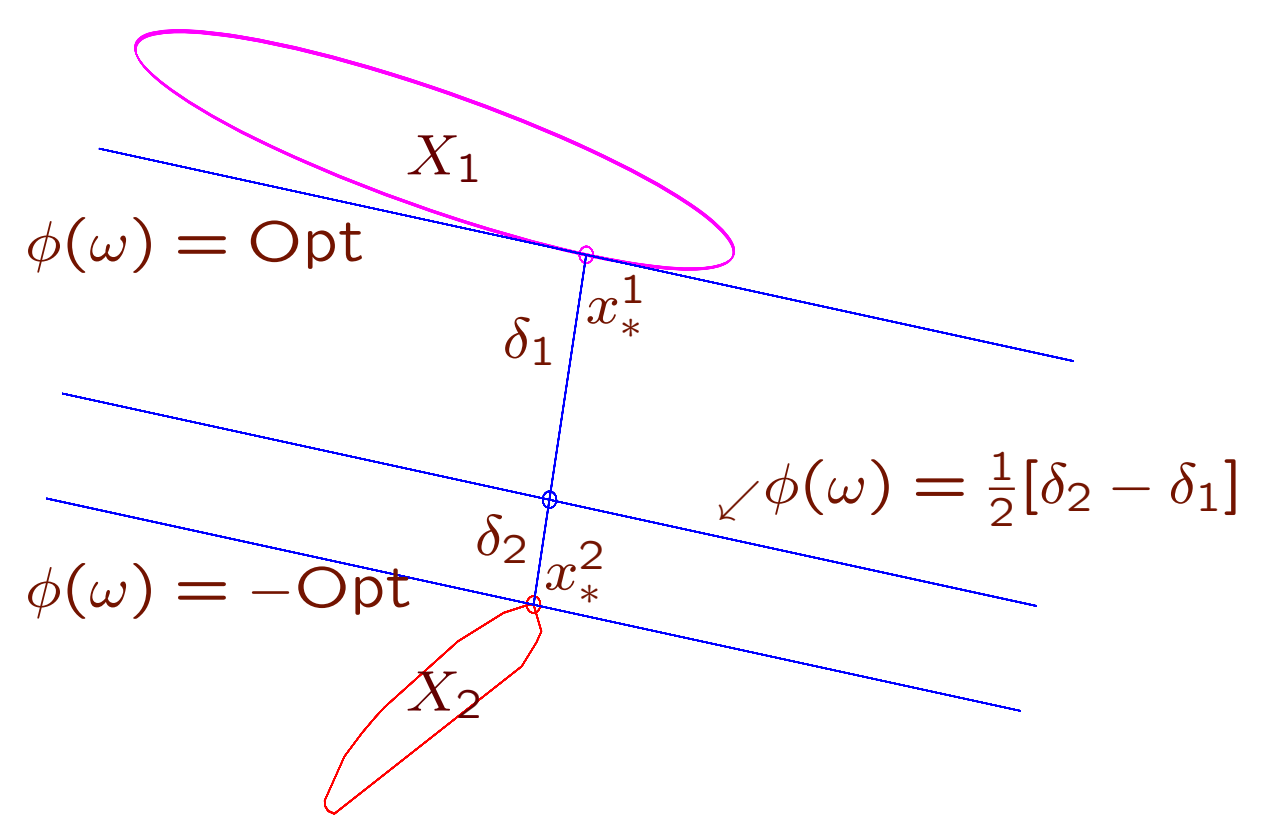

- Given $\delta_{1} \geq 0, \delta_{2} \geq 0$ with $\delta_{1}+\delta_{2}=20 \mathrm{pt}, \phi(\cdot)$ specifies simple Euclidean Separation Test $\mathcal{T}$ induced by $X_{1}, X_{2}, \delta_{1}, \delta_{2}$ :

$$
\mathcal{T}(\omega)= \begin{cases}\{1\}, & \phi(\omega) \geq \frac{1}{2}\left[\delta_{2}-\delta_{1}\right] \\ \{2\}, & \text { otherwise }\end{cases}
$$


Observation: Let $\xi \sim \mathcal{N}(0, I)$. Given observation $\omega=x+\xi$ the Euclidean Separation Test $\mathcal{T}$ decides on the hypotheses

$$
H_{1}: x \in X_{1}, H_{2}: x \in X_{2}
$$

with risks satisfying

$$
\begin{aligned}
& \operatorname{Risk}_{1}\left[\mathcal{T} \mid H_{1}, H_{2}\right] \leq \operatorname{Prob}_{\zeta \sim N(0,1)}\left\{\zeta \geq \delta_{1}\right\}=\frac{1}{2} \operatorname{erfc}\left(\delta_{1} / \sqrt{2}\right) \\
& \operatorname{Risk}_{2}\left[\mathcal{T} \mid H_{1}, H_{2}\right] \leq \operatorname{Prob}_{\zeta \sim N(0,1)}\left\{\zeta \geq \delta_{2}\right\}=\frac{1}{2} \operatorname{erfc}\left(\delta_{2} / \sqrt{2}\right) .
\end{aligned}
$$

In addition, when $\delta_{1}=\delta_{2}=\mathrm{Opt}, \mathcal{T}$ is the minimum risk test deciding on $H_{1}$, $\mathrm{H}_{2}$. The risk of this test is

$$
\operatorname{Risk}\left[\mathcal{T} \mid H_{1}, H_{2}\right]=\operatorname{Prob}_{\zeta \sim N(0,1)}\{\zeta \geq \text { Opt }\}=\frac{1}{2} \operatorname{erfc}(\mathrm{Opt} / \sqrt{2}) .
$$

Note: By Neyman-Pearson lemma, this risk is the smallest attainable under circumstances. 


\section{From Pairwise to Multiple Hypotheses Testing}

Situation: We are given $L$ families of probability distributions $\mathcal{P}_{\ell}, 1 \leq \ell \leq L$, on observation space $\Omega$, and observe a realization of random variable $\omega \sim P$ taking values in $\Omega$. Given $\omega$, we want to decide on $L$ hypotheses

$$
H_{\ell}: P \in \mathcal{P}_{\ell}, 1 \leq \ell \leq L .
$$

Our ideal goal would be to find a low-risk simple test deciding on these hypotheses.

Note: It may happen that the "ideal goal" is not achievable, for instance, when some pairs of families $\mathcal{P}_{\ell}$ have nonempty intersections. When $\mathcal{P}_{\ell} \cap \mathcal{P}_{\ell^{\prime}} \neq \emptyset$ for some $\ell \neq \ell^{\prime}$, there is no way to decide on the hypotheses with risk $<1 / 2$.

Fortunately, impossibility to decide reliably on all L hypotheses "individually" does not mean that no meaningful inferences can be done. 


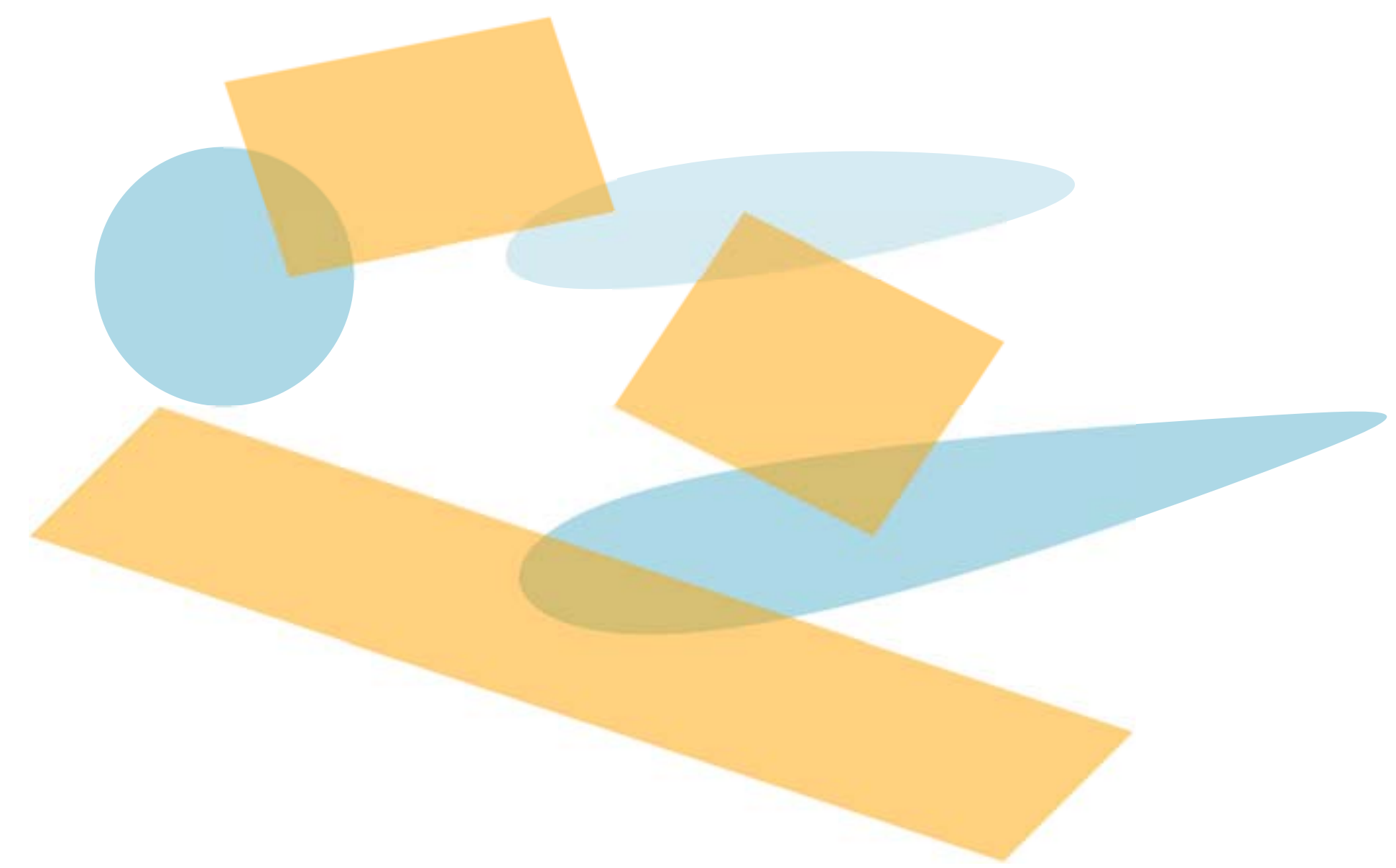

When handling multiple hypotheses which cannot be reliably decided upon "as they are," it makes sense to speak about testing the hypotheses "up to closeness." 


$$
\omega \sim P, \quad H_{\ell}: P \in \mathcal{P}_{\ell}, 1 \leq \ell \leq L
$$

Closeness relation $\mathcal{C}$ on $L$ hypotheses $H_{1}, \ldots, H_{L}$ is defined as a set of pairs $\left(\ell, \ell^{\prime}\right)$ with $1 \leq \ell, \ell^{\prime} \leq L$; we interpret the relation $\left(\ell, \ell^{\prime}\right) \in \mathcal{C}$ as the fact that the hypotheses $H_{\ell}$ and $H_{\ell}^{\prime}$ are close to each other.

We always assume that

- $\mathcal{C}$ contains all "diagonal pairs" $(\ell, \ell), 1 \leq \ell \leq L$ : (every hypothesis is close to itself")

- $\left(\ell, \ell^{\prime}\right) \in \mathcal{C}$ if and only if $\left(\ell^{\prime}, \ell\right) \in \mathcal{C}$ ("closeness is symmetric")

Note: By symmetry of $\mathcal{C}$, the relation $\left(\ell, \ell^{\prime}\right) \in \mathcal{T}$ is in fact a property of unordered pair $\left\{\ell, \ell^{\prime}\right\}$. 


\section{"Up to closeness" risks}

Let $\mathcal{T}$ be a test deciding on $H_{1}, \ldots, H_{L}$; given observation $\omega, \mathcal{T}$ accepts all hypotheses $H_{\ell}$ with indexes $\ell \in \mathcal{T}(\omega)$ and rejects all other hypotheses.

We say that $\ell$-th partial $\mathcal{C}$-risk of test $\mathcal{T}$ is $\leq \epsilon$, if whenever $H_{\ell}$ is true: $\omega \sim P \in$ $\mathcal{P}_{\ell}$, the $P$-probability of the event

$\mathcal{T}$ accepts $H_{\ell}: \ell \in \mathcal{T}(\omega)$ and all hypotheses $H_{\ell^{\prime}}$ accepted by $\mathcal{T}$ are

$\mathcal{C}$-close to $H_{\ell}:\left(\ell, \ell^{\prime}\right) \in \mathcal{C} \forall \ell^{\prime} \in \mathcal{T}(\omega)$

is at least $1-\epsilon$.

$\ell$-th partial $\mathcal{C}$-risk of $\mathcal{T}$ is the smallest $\epsilon$ with the outlined property:

$\operatorname{Risk}_{\ell}^{\mathcal{C}}\left[\mathcal{T} \mid H_{1}, \ldots, H_{L}\right]=\sup _{P \in \mathcal{P}_{\ell}} \operatorname{Prob}_{\omega \sim P}\left\{[\ell \notin \mathcal{T}(\omega)]\right.$ or $\left.\left[\exists \ell^{\prime} \in \mathcal{T}(\omega):\left(\ell, \ell^{\prime}\right) \notin \mathcal{C}\right]\right\}$

$\mathcal{C}$-risk of $\mathcal{T}$ is the largest of the partial $\mathcal{C}$-risks of the test:

$$
\operatorname{Risk}^{\mathcal{C}}\left[\mathcal{T} \mid H_{1}, \ldots, H_{L}\right]=\max _{1 \leq \ell \leq L} \operatorname{Risk}_{\ell}^{\mathcal{C}}\left[\mathcal{T} \mid H_{1}, \ldots, H_{L}\right] .
$$




\section{Multiple Hypothesis Testing via Pairwise Tests}

Assume that for every unordered pair $\left\{\ell, \ell^{\prime}\right\}$ with $\left(\ell, \ell^{\prime}\right) \notin \mathcal{C}$ we are given a simple test $\mathcal{T}_{\left\{\ell, \ell^{\prime}\right\}}$ deciding on $H_{\ell}$ vs. $H_{\ell^{\prime}}$ via observation $\omega$.

\section{Construction:}

- For $\left(\ell, \ell^{\prime}\right) \notin \mathcal{C}$, so that $\ell \neq \ell^{\prime}$, we define function $T_{\ell \ell^{\prime}}(\omega)$ as follows:

$$
T_{\ell \ell^{\prime}}(\omega)=\left\{\begin{array}{rl}
1, & \mathcal{T}_{\left\{\ell, \ell^{\prime}\right\}}(\omega)=\{\ell\} \\
-1, & \mathcal{T}_{\left\{\ell, \ell^{\prime}\right\}}(\omega)=\left\{\ell^{\prime}\right\}
\end{array} .\right.
$$

Since $\mathcal{T}_{\left\{\ell, \ell^{\prime}\right\}}$ is a simple test, $T_{\ell \ell^{\prime}}(\cdot)$ is well defined and takes values \pm 1 .

- For $\left(\ell, \ell^{\prime}\right) \in \mathcal{C}$, we set $T_{\ell \ell^{\prime}}(\cdot) \equiv 0$.

By construction, we have $T_{\ell \ell^{\prime}}(\omega) \equiv-T_{\ell^{\prime} \ell}(\omega), 1 \leq \ell, \ell^{\prime} \leq L$.

- The test $\mathcal{T}$ is as follows: given observation $\omega$, we build $L \times L$ matrix $T(\omega)=\left[T_{\ell \ell^{\prime}}(\omega)\right]$ and accept exactly those of the hypotheses $H_{\ell}$ for which $\ell$-th row in $T(\omega)$ is nonnegative, that is, all tests $\mathcal{T}_{\left\{\ell, \ell^{\prime}\right\}}$ with $\left(\ell, \ell^{\prime}\right) \notin \mathcal{C}$ accept $H_{\ell}$, observation being $\omega$. 
Example: $L=4, \quad \mathcal{C}=\{(1,1),(2,2),(3,3),(4,4),\{1,2\},\{2,3\},\{3,4\}\}$ Suppose that given tests $\mathcal{T}_{\{1,3\}}, \mathcal{T}_{\{1,4\}}, \mathcal{T}_{\{2,4\}}$ and observation $\omega$ :

- $\mathcal{T}_{\{1,3\}}$ accepts $H_{1}, \mathcal{T}_{\{1,4\}}$ accepts $H_{1}, \mathcal{T}_{\{2,4\}}$ accepts $H_{4}$, so we get

$\Rightarrow$ Aggregated test $\mathcal{T}$ accepts $H_{1}$

$$
T(\omega)=\left[\begin{array}{c|c|c|c}
0 & 0 & +1 & +1 \\
\hline 0 & 0 & 0 & -1 \\
\hline-1 & 0 & 0 & 0 \\
\hline-1 & +1 & 0 & 0
\end{array}\right]
$$

- when $\mathcal{T}_{\{1,3\}}$ accepts $H_{1}, \mathcal{T}_{\{1,4\}}$ accepts $H_{1}, \mathcal{T}_{\{2,4\}}$ accepts $H_{2}$, we get

$\Rightarrow$ Aggregated test $\mathcal{T}$ accepts $H_{1}$ and $H_{2}$

$$
T(\omega)=\left[\begin{array}{c|c|c|c}
0 & 0 & +1 & +1 \\
\hline 0 & 0 & 0 & +1 \\
\hline-1 & 0 & 0 & 0 \\
\hline-1 & -1 & 0 & 0
\end{array}\right]
$$


Observation: When $\mathcal{T}$ accepts some hypothesis $H_{\ell}$, all hypotheses accepted by $\mathcal{T}$ are $\mathcal{C}$-close to $H_{\ell}$.

Indeed, if $\ell$-th row in $T(\omega)$ is nonnegative and $\ell^{\prime}$ is not $\mathcal{C}$-close to $\ell$, we have

$$
\begin{aligned}
& T_{\ell \ell^{\prime}}(\omega) \geq 0 \text { and } T_{\ell \ell^{\prime}}(\omega) \in\{-1,1\} \\
\Rightarrow & T_{\ell \ell^{\prime}}(\omega)=1 \\
\Rightarrow & T_{\ell^{\prime} \ell}(\omega)=-T_{\ell \ell^{\prime}}(\omega)=-1 \\
\Rightarrow & \ell^{\prime} \text {-th row in } T(\omega) \text { is not nonnegative } \Rightarrow \ell^{\prime} \text { is not accepted }
\end{aligned}
$$

Risk analysis. For $\left(\ell, \ell^{\prime}\right) \notin \mathcal{C}$, let

$$
\begin{aligned}
\epsilon_{\ell \ell^{\prime}} & =\operatorname{Risk}_{1}\left[\mathcal{T}_{\left\{\ell, \ell^{\prime}\right\}} \mid H_{\ell}, H_{\ell^{\prime}}\right]=\sup _{P \in \mathcal{P}_{\ell}} \operatorname{Prob}_{\omega \sim P}\left\{\ell \notin \mathcal{T}_{\left\{\ell, \ell^{\prime}\right\}}(\omega)\right\} \\
& =\sup _{P \in \mathcal{P}_{\ell}} \operatorname{Prob}_{\omega \sim P}\left\{T_{\ell \ell^{\prime}}(\omega)=-1\right\}=\sup _{P \in \mathcal{P}_{\ell}} \operatorname{Prob}_{\omega \sim P}\left\{T_{\ell^{\prime} \ell}(\omega)=1\right\} \\
& =\sup _{P \in \mathcal{P}_{\ell}} \operatorname{Prob}_{\omega \sim P}\left\{\ell^{\prime} \in \mathcal{T}_{\left\{\ell, \ell^{\prime}\right\}}(\omega)\right\}=\operatorname{Risk}_{2}\left[\mathcal{T}_{\left\{\ell, \ell^{\prime}\right\}} \mid H_{\ell^{\prime}}, H_{\ell}\right] .
\end{aligned}
$$


Proposition. One has

$$
\operatorname{Risk}_{\ell}^{\mathcal{C}}\left[\mathcal{T} \mid H_{1}, \ldots, H_{L}\right] \leq \epsilon_{\ell}:=\sum_{\ell^{\prime}:\left(\ell, \ell^{\prime}\right) \notin \mathcal{C}} \epsilon_{\ell \ell^{\prime}}
$$

Indeed, let us fix $\ell$, and let $H_{\ell}$ be true. Let $P \in \mathcal{P}_{\ell}$ be the distribution of observation $\omega$, and let $I=\left\{\ell^{\prime} \leq L:\left(\ell, \ell^{\prime}\right) \notin \mathcal{C}\right\}$. For $\ell^{\prime} \in I$, let $\mathcal{E}_{\ell^{\prime}}$ be the event $\left\{\omega: T_{\ell \ell^{\prime}}(\omega)=-1\right\}$. We have $\operatorname{Prob}_{\omega \sim P}\left(\mathcal{E}_{\ell^{\prime}}\right) \leq \epsilon_{\ell \ell^{\prime}}$ (by definition of $\left.\epsilon_{\ell \ell^{\prime}}\right) \Rightarrow \operatorname{Prob}_{\omega \sim P}(\underbrace{\cup_{\ell^{\prime} \in I^{\prime}} \mathcal{E}_{\ell^{\prime}}}_{\mathcal{E}}) \leq \epsilon_{\ell}$

When the event $\mathcal{E}$ does not take place, we have $T_{\ell \ell^{\prime}}(\omega)=1$ for all $\ell^{\prime} \in I$

$\Rightarrow T_{\ell \ell^{\prime}}(\omega) \geq 0$ for all $\ell^{\prime}, 1 \leq \ell^{\prime} \leq L$

$\Rightarrow \ell \in \mathcal{T}(\omega)$

$\Rightarrow$ (by Observation) $\{\ell \in \mathcal{T}(\omega)\} \&\left\{\left(\ell, \ell^{\prime}\right) \in \mathcal{C} \forall \ell^{\prime} \in \mathcal{T}(\omega)\right\}$.

By definition of partial $\mathcal{C}$-risk, we get

$$
\operatorname{Risk}_{\ell}^{\mathcal{C}}\left[\mathcal{T} \mid H_{1}, \ldots, H_{L}\right] \leq \operatorname{Prob}_{\omega \sim P}(\mathcal{E}) \leq \epsilon_{\ell} .
$$




\section{Testing Multiple Hypotheses via Euclidean Separation}

Situation: We are given $L$ nonempty, closed and bounded convex sets $X_{\ell} \subset$ $\mathbb{R}^{d}, 1 \leq \ell \leq L$ and an observation $\omega=x+\xi$, so that

- $x$ is a deterministic sequence of signals, and $\xi \sim \mathcal{N}(0, I)$.

Given $\omega^{K}$, we want to decide up to closeness $\mathcal{C}$ on $L$ hypotheses $H_{\ell}: x \in X_{\ell}$.

Equivalently: Sets $X_{\ell} \subset \mathbb{R}^{d}$ specify $L$ families of distributions

$$
\mathcal{P}_{\ell}=\left\{\mathcal{N}(x, I): x \in X_{\ell}\right\}, \quad 1 \leq \ell \leq L
$$

Given $\omega$, we want to decide, up to closeness $\mathcal{C}$, on $L$ hypotheses

$$
H_{\ell}: P \in \mathcal{P}_{\ell}, \quad 1 \leq \ell \leq L
$$

on the distribution $P^{K}$ of observation $\omega^{K}$.

We intend to assemble pairwise Euclidean separation tests.

Standing Assumption: Whenever $\ell, \ell^{\prime}$ are not $\mathcal{C}$-close: $\left(\ell, \ell^{\prime}\right) \notin \mathcal{C}$, the sets $X_{\ell}, X_{\ell^{\prime}}$ do not intersect. 
Building blocks. For $\left(\ell, \ell^{\prime}\right) \notin \mathcal{C}$, we solve convex optimization problems

$$
\mathrm{Opt}_{\ell \ell^{\prime}}=\min _{u \in X_{\ell}, v \in X_{\ell^{\prime}}} \frac{1}{2}\|u-v\|_{2}
$$

By Standing Assumption, $\mathrm{Opt}_{\ell \ell^{\prime}}>0$. Optimal solution $\left(u_{*}, v_{*}\right)$ to $\left(P_{\ell \ell^{\prime}}\right)$ defines affine functions

$$
\phi_{\ell \ell^{\prime}}(\omega)=e_{\ell \ell^{\prime}}^{T} \omega-c_{\ell \ell^{\prime}} \quad e_{\ell \ell^{\prime}}=\frac{u_{*}-v_{*}}{\left\|u_{*}-v_{*}\right\|_{2}}, c_{\ell \ell^{\prime}}=\frac{1}{2} e_{\ell \ell^{\prime}}^{T}\left[u_{*}+v_{*}\right]
$$

Note: We have $\phi_{\ell \ell^{\prime}}(\cdot) \equiv-\phi_{\ell^{\prime} \ell}(\cdot)$ for all $\left(\ell, \ell^{\prime}\right) \notin \mathcal{C}$.

As we know, whenever $\delta_{\ell \ell^{\prime}} \geq 0, \delta_{\ell^{\prime} \ell} \geq 0$ satisfy

$$
20 \mathrm{pt}_{\ell \ell^{\prime}}=\delta_{\ell \ell^{\prime}}+\delta_{\ell^{\prime} \ell}
$$

it holds

$$
\begin{aligned}
& \forall u \in X_{\ell}: \operatorname{Prob}_{u}\left\{\phi(u+\xi)<\frac{1}{2}\left[\delta_{\ell^{\prime} \ell}-\delta_{\ell \ell^{\prime}}\right]\right\} \leq \Gamma\left(\delta_{\ell \ell^{\prime}}\right):=\int_{\delta_{\ell \ell^{\prime}}}^{\infty} \gamma(s) d s, \\
& \forall v \in X_{\ell^{\prime}}: \operatorname{Prob}_{v}\left\{\phi(v+\xi) \geq \frac{1}{2}\left[\delta_{\ell^{\prime} \ell}-\delta_{\ell \ell^{\prime}}\right]\right\} \leq \Gamma\left(\delta_{\ell^{\prime} \ell}\right):=\int_{\delta_{\ell^{\prime} \ell}}^{\infty} \gamma(s) d s
\end{aligned}
$$

where $\gamma(\cdot)$ is the standard normal density. 


$$
\begin{gathered}
\ell, \ell^{\prime}:\left(\ell, \ell^{\prime}\right) \notin \mathcal{C} \Rightarrow \operatorname{Opt}_{\ell^{\prime}}=\min _{u \in X_{\ell}, v \in X_{\ell^{\prime}}} \frac{1}{2}\|u-v\|_{2}>0=\text { Opt }_{\ell^{\prime} \ell} \\
\Rightarrow u_{*}, v_{*}, \quad \phi_{\ell \ell^{\prime}}(\omega)=e_{\ell \ell^{\prime}}^{T} \omega-c_{\ell \ell^{\prime}} \equiv-\phi_{\ell^{\prime} \ell^{\prime}}(\omega)\left[e_{\ell \ell^{\prime}}=\frac{u_{*}-v_{*}}{\left\|u_{*}-v_{*}\right\|_{2}}, c_{\ell \ell^{\prime}}=\frac{1}{2} e_{\ell \ell^{\prime}}^{T}\left[u_{*}+v_{*}\right]\right] \\
\quad \delta_{\ell \ell^{\prime}} \geq 0, \quad \delta_{\ell^{\prime} \ell} \geq 0, \quad 2 O \operatorname{pt}_{\ell \ell^{\prime}}=\delta_{\ell \ell^{\prime}}+\delta_{\ell^{\prime} \ell} \\
\forall u \in X_{\ell}: \operatorname{Prob}_{\xi \sim P}\left\{\phi(u+\xi)<\frac{1}{2}\left[\delta_{\ell^{\prime} \ell}-\delta_{\ell \ell^{\prime}}\right]\right\} \leq \Gamma\left(\delta_{\ell \ell^{\prime}}\right):=\int_{\delta_{\ell \ell^{\prime}}}^{\infty} \gamma(s) d s \\
\forall v \in X_{\ell^{\prime}}: \quad \operatorname{Prob}_{\xi \sim P}\left\{\phi(v+\xi) \geq \frac{1}{2}\left[\delta_{\ell^{\prime} \ell}-\delta_{\ell \ell^{\prime}}\right]\right\} \leq \Gamma\left(\delta_{\ell^{\prime} \ell}\right):=\int_{\delta_{\ell^{\prime} \ell}}^{\infty} \gamma(s) d s
\end{gathered}
$$

\section{Assembling building blocks}

- For $\ell, \ell^{\prime}$ with $\left(\ell, \ell^{\prime}\right) \notin \mathcal{C}$ we select $\delta_{\ell \ell^{\prime}}$ satisfying $(*)$, thus arriving at simple tests

$$
\mathcal{T}_{\left\{\ell, \ell^{\prime}\right\}}(\omega)= \begin{cases}\{\ell\}, & \phi_{\ell \ell^{\prime}}(\omega) \geq \frac{1}{2}\left[\delta_{\ell^{\prime} \ell}-\delta_{\ell \ell^{\prime}}\right] \\ \left\{\ell^{\prime}\right\}, & \phi_{\ell \ell^{\prime}}(\omega)<\frac{1}{2}\left[\delta_{\ell^{\prime} \ell}-\delta_{\ell \ell^{\prime}}\right]\end{cases}
$$

- Further, we use out general construction to assemble tests $\left\{\mathcal{T}_{\left\{\ell, \ell^{\prime}\right\}}\right.$ : $\left.\left(\ell, \ell^{\prime}\right) \notin \mathcal{C}\right\}$ into test $\mathcal{T}$ deciding on $H_{1}, \ldots, H_{L}$

Note: By (!), associated with tests $\mathcal{T}_{\left\{\ell, \ell^{\prime}\right\}}$ quantities $\epsilon_{\ell \ell^{\prime}}$ satisfy the relations $\epsilon_{\ell \ell^{\prime}} \leq \Gamma\left(\delta_{\ell \ell^{\prime}}\right):=\int_{\delta_{\ell \ell^{\prime}}}^{\infty} \gamma(s) d s$, whence $\operatorname{Risk}_{\ell}^{\mathcal{C}}\left[\mathcal{T} \mid H_{1}, \ldots, H_{L}\right] \leq \sum_{\ell^{\prime}:\left(\ell, \ell^{\prime}\right) \notin \mathcal{C}} \Gamma\left(\delta_{\ell \ell^{\prime}}\right)$. 


$$
\begin{gathered}
\ell, \ell^{\prime}:\left(\ell, \ell^{\prime}\right) \notin \mathcal{C} \Rightarrow \operatorname{Opt}_{\ell \ell^{\prime}}=\min _{u \in X_{\ell}, v \in X_{\ell^{\prime}}} \frac{1}{\|}\|u-v\|_{2} \\
\Rightarrow \delta_{\ell \ell^{\prime}} \geq 0, \delta_{\ell^{\prime} \ell} \geq 0,2 \mathrm{Opt}_{\ell \ell^{\prime}}=\delta_{\ell \ell^{\prime}}+\delta_{\ell^{\prime} \ell} \\
\Rightarrow \mathcal{T}: \operatorname{Risk}_{\ell}^{\mathcal{C}}\left[\mathcal{T} \mid H_{1}, \ldots, H_{L}\right] \leq \sum_{\ell^{\prime}:\left(\ell, \ell^{\prime}\right) \notin \mathcal{C}} \Gamma\left(\delta_{\ell \ell^{\prime}}\right), \Gamma(\delta)=\int_{\delta}^{\infty} \gamma(s) d s
\end{gathered}
$$

\section{Refining the construction.}

We can try to optimize the "profile" of (upper bounds on) partial $\mathcal{C}$-risks of the test $\mathcal{T}$ over the "free parameters" $\delta_{\ell \ell^{\prime}},\left(\ell, \ell^{\prime}\right) \notin \mathcal{C}$, of the construction.

A model here may be as follows: given nonnegative weight matrix $W$ and nonnegative vectors $\alpha$ and $\beta$, we want to minimize "scale factor" $t$ under the constraint

$$
W \operatorname{vec}\left[\operatorname{Risk}_{\ell}^{\mathcal{C}}\left[\mathcal{T} \mid H_{1}, \ldots, H_{L}\right]\right]_{\ell=1}^{L} \leq \alpha+t \beta
$$

This problem can be safely approximated by the optimization problem

$$
\min _{\left\{\delta_{\ell^{\prime}}\right\}, t}\left\{t: \begin{array}{ll}
W \operatorname{vec}\left[\sum_{\ell^{\prime}:\left(\ell, \ell^{\prime}\right.}\right) \notin \mathcal{C} & \left.\Gamma\left(\delta_{\ell \ell^{\prime}}\right)\right]_{\ell=1}^{L} \leq \alpha+t \beta \\
& \delta_{\ell \ell^{\prime}} \geq 0, \delta_{\ell \ell^{\prime}}+\delta_{\ell^{\prime} \ell}=20 \mathrm{pt}_{\ell \ell^{\prime}},\left(\ell, \ell^{\prime}\right) \notin \mathcal{C}
\end{array}\right\}
$$

Note: When $\gamma(\cdot)$ is nonincreasing on $\mathbb{R}_{+}$(as is the case for Gaussian density), function $\Gamma(\delta)=\int_{\delta}^{\infty} \gamma(s) d s$ is convex on $\mathbb{R}_{+}$

$\Rightarrow(\#)$ is an explicit Convex Programming problem! 


\section{HYPOTHESIS TESTING, II}

- Detector-Based Tests

- Detectors \& Detector-Based Pairwise Tests

- Testing "up to Closeness"

- Simple Observation Schemes

- Minimum Risk Detectors

- Near-Optimal Tests

- Applications

- Recovery of Functionals

- Sequential Hypothesis Testing

- Measurement Design 


\section{Detectors \& Detector-Based Pairwise Tests}

Situation: Given two families $\mathcal{P}_{1}, \mathcal{P}_{2}$ of probability distributions on a given observation space $\Omega$ and an observation $\omega \sim P$ with $P$ known to belong to $\mathcal{P}_{1} \cup \mathcal{P}_{2}$, we want to decide whether

$$
P \in \mathcal{P}_{1} \text { (hypothesis } H_{1} \text { ) or } P \in \mathcal{P}_{2} \text { (hypothesis } H_{2} \text { ). }
$$

Detectors. A detector is a function $\phi: \Omega \rightarrow \mathbb{R}$. Risks of a detector $\phi$ w.r.t. $\mathcal{P}_{1}$ and $\mathcal{P}_{2}$ are defined as

$$
\begin{aligned}
& \operatorname{Risk}_{1}\left[\phi \mid \mathcal{P}_{1}, \mathcal{P}_{2}\right]=\sup _{P \in \mathcal{P}_{1} \Omega} \int \mathrm{e}^{-\phi(\omega)} P(d \omega), \\
& \operatorname{Risk}_{2}\left[\phi \mid \mathcal{P}_{1}, \mathcal{P}_{2}\right]=\sup _{P \in \mathcal{P}_{2} \Omega} \int \mathrm{e}^{\phi(\omega)} P(d \omega) \\
& \operatorname{Risk}_{1}\left[\phi \mid \mathcal{P}_{1}, \mathcal{P}_{2}\right]=\operatorname{Risk}_{2}\left[-\phi \mid \mathcal{P}_{2}, \mathcal{P}_{1}\right]
\end{aligned}
$$


Simple test $\mathcal{T}_{\phi}$ associated with detector $\phi$, given observation $\omega$,

- accepts $H_{1}$ when $\phi(\omega) \geq 0$

- accepts $H_{2}$ when $\phi(\omega)<0$.

Immediate observation:

$$
\begin{aligned}
& \operatorname{Risk}_{1}\left[\mathcal{T}_{\phi} \mid H_{1}, H_{2}\right] \leq \operatorname{Risk}_{1}\left[\phi \mid \mathcal{P}_{1}, \mathcal{P}_{2}\right] \\
& \operatorname{Risk}_{2}\left[\mathcal{T}_{\phi} \mid H_{1}, H_{2}\right] \leq \operatorname{Risk}_{2}\left[\phi \mid \mathcal{P}_{1}, \mathcal{P}_{2}\right]
\end{aligned}
$$

Indeed, $\operatorname{Prob}_{\omega \sim P}\{\omega: \psi(\omega) \geq 0\} \leq \int \mathrm{e}^{\psi(\omega)} P(d \omega)$. 


$$
\begin{aligned}
& \operatorname{Risk}_{1}\left[\phi \mid \mathcal{P}_{1}, \mathcal{P}_{2}\right]=\sup _{P \in \mathcal{P}_{1} \Omega} \int_{\Omega} \mathrm{e}^{-\phi(\omega)} P(d \omega) \\
& \operatorname{Risk}_{2}\left[\phi \mid \mathcal{P}_{1}, \mathcal{P}_{2}\right]=\sup _{P \in \mathcal{P}_{2} \Omega} \int^{\phi(\omega)} P(d \omega)
\end{aligned}
$$

\section{Elementary Calculus of Detectors}

- Renormalization (shift): $\phi(\cdot) \Rightarrow \phi_{a}(\cdot)=\phi(\cdot)-a$

$$
\Rightarrow\left\{\begin{array}{l}
\operatorname{Risk}_{1}\left[\phi_{a} \mid \mathcal{P}_{1}, \mathcal{P}_{2}\right]=\mathrm{e}^{a} \operatorname{Risk}_{1}\left[\phi \mid \mathcal{P}_{1}, \mathcal{P}_{2}\right] \\
\operatorname{Risk}_{2}\left[\phi_{a} \mid \mathcal{P}_{1}, \mathcal{P}_{2}\right]=\mathrm{e}^{-a} \operatorname{Risk}_{2}\left[\phi \mid \mathcal{P}_{1}, \mathcal{P}_{2}\right]
\end{array}\right.
$$

$\Rightarrow$ What matters, is the product

$$
\left[\operatorname{Risk}\left[\phi \mid \mathcal{P}_{1}, \mathcal{P}_{2}\right]\right]^{2}:=\operatorname{Risk}_{1}\left[\phi \mid \mathcal{P}_{1}, \mathcal{P}_{2}\right] \operatorname{Risk}_{2}\left[\phi \mid \mathcal{P}_{1}, \mathcal{P}_{2}\right]
$$

of partial risks of a detector. Shifting detector by a constant, we can redistribute this product between factors as we want, e.g., make the detector balanced:

$$
\operatorname{Risk}\left[\phi \mid \mathcal{P}_{1}, \mathcal{P}_{2}\right]=\operatorname{Risk}_{1}\left[\phi \mid \mathcal{P}_{1}, \mathcal{P}_{2}\right]=\operatorname{Risk}_{2}\left[\phi \mid \mathcal{P}_{1}, \mathcal{P}_{2}\right] .
$$




\section{Detectors are well-suited to passing to multiple observations.}

For $1 \leq k \leq K$, let

- $\mathcal{P}_{1, k}, \mathcal{P}_{2, k}$ be families of probability distributions on observation spaces $\Omega_{k}$,

- $\phi_{k}$ be detectors on $\Omega_{k}$.

Families $\left\{\mathcal{P}_{1, k}, \mathcal{P}_{2, k}\right\}_{k=1}^{K}$ give rise to families of product distributions

$$
\mathcal{P}_{\chi}^{\oplus, K}=\bigoplus_{k=1}^{K} \mathcal{P}_{\chi, k}:=\left\{P^{K}=P_{1} \times \ldots \times P_{K}: P_{k} \in \mathcal{P}_{\chi . k}, 1 \leq k \leq K\right\} .
$$

on $\Omega^{K}=\Omega_{1} \times \ldots \times \Omega_{K}, \chi=1,2$. We call $\mathcal{P}_{\chi}^{K}$ direct products of $\mathcal{P}_{\chi, k}$.

Detectors $\phi_{1}, . ., \phi_{K}$ give rise to detector $\phi^{K}$ on $\Omega^{K}$ :

$$
\phi^{K}(\underbrace{\omega_{1}, \ldots, \omega_{K}}_{\omega^{K}})=\sum_{k=1}^{K} \phi_{k}\left(\omega_{k}\right) .
$$

Observation: For $\chi=1,2$, we have

$$
\operatorname{Risk}_{\chi}\left[\phi^{K} \mid \mathcal{P}_{1}^{\oplus, K}, \mathcal{P}_{2}^{\oplus, K}\right]=\prod_{k=1}^{K} \operatorname{Risk}_{\chi}\left[\phi_{k} \mid \mathcal{P}_{1, k}, \mathcal{P}_{2, k}\right]
$$


From pairwise detectors to detectors for unions. Assume that we are given an observation space $\Omega$ along with

- $R$ families $\mathcal{R}_{r}, r=1, \ldots, R$ of "red" probability distributions on $\Omega$,

- $B$ families $\mathcal{B}_{b}, b=1, \ldots, B$ of "brown" probability distributions on $\Omega$,

- pairwise detectors $\phi_{r b}(\cdot), 1 \leq r \leq R, 1 \leq b \leq B$.

Let us aggregate the red and the brown families into 2 groups as follows

$$
\mathcal{R}=\bigcup_{r=1}^{R} \mathcal{R}_{r}, \mathcal{B}=\bigcup_{b=1}^{B} \mathcal{B}_{b}
$$

and assemble detectors $\phi_{r b}$ into a single detector

$$
\phi(\omega)=\max _{r \leq R} \min _{b \leq B} \phi_{r b}(\omega) .
$$

- We have

$$
\begin{aligned}
\operatorname{Risk}_{1}[\phi \mid \mathcal{R}, \mathcal{B}] & \leq \max _{r \leq R} \sum_{b \leq B} \operatorname{Risk}_{1}\left[\phi_{r b} \mid \mathcal{R}_{r}, \mathcal{B}_{b}\right] \\
\operatorname{Risk}_{2}[\phi \mid \mathcal{R}, \mathcal{B}] & \leq \max _{b \leq B} \sum_{r \leq R} \operatorname{Risk}_{2}\left[\phi_{r b} \mid \mathcal{R}_{r}, \mathcal{B}_{b}\right]
\end{aligned}
$$




$$
\begin{aligned}
& \operatorname{Risk}_{1}[\phi \mid \mathcal{R}, \mathcal{B}] \leq \max _{r \leq R} \sum_{b \leq B} \operatorname{Risk}_{1}\left[\phi_{r b} \mid \mathcal{R}_{r}, \mathcal{B}_{b}\right], \\
& \operatorname{Risk}_{2}[\phi \mid \mathcal{R}, \mathcal{B}] \leq \max _{b \leq B} \sum_{r \leq R} \operatorname{Risk}_{2}\left[\phi_{r b} \mid \mathcal{R}_{r}, \mathcal{B}_{b}\right],
\end{aligned}
$$

Indeed, because $-\max _{r} \min _{b} \phi_{r b}(\omega)=\min _{r} \max _{b}-\phi_{r b}(\omega)$,

$$
\begin{aligned}
P \in \mathcal{R}_{r_{*}} \Rightarrow \int \mathrm{e}^{-\phi(\omega) P(d \omega)} & =\int \mathrm{e}^{\min _{r} \max _{b}\left[-\phi_{r b}(\omega)\right]} P(d \omega) \\
& \leq \int \mathrm{e}^{\max _{b}\left[-\phi_{r_{*} b}(\omega)\right]} P(d \omega) \leq \sum_{b} \int \mathrm{e}^{-\phi_{r_{*} b}(\omega)} P(d \omega) \\
& \leq \sum_{b} \operatorname{Risk}_{1}\left[\phi_{r_{*} b} \mid \mathcal{R}_{r_{*}}, \mathcal{B}_{b}\right] \\
\Rightarrow \operatorname{Risk}_{1}[\phi \mid \mathcal{R}, \mathcal{B}] & \leq \max _{r \leq R} \sum_{b \leq B} \operatorname{Risk}_{1}\left[\phi_{r b} \mid \mathcal{R}_{r}, \mathcal{B}_{b}\right] .
\end{aligned}
$$

In the same way,

$$
\begin{aligned}
P \in \mathcal{B}_{b_{*}} \Rightarrow \int \mathrm{e}^{\phi(\omega)} P(d \omega) & =\int \mathrm{e}^{\max _{r} \min _{b}\left[\phi_{r b}(\omega)\right]} P(d \omega) \leq \int \mathrm{e}^{\max _{r} \phi_{r b_{*}}(\omega)} P(d \omega) \\
& \leq \sum_{r} \int \mathrm{e}^{\phi_{r b_{*}}(\omega)} P(d \omega) \leq \sum_{r} \operatorname{Risk}_{2}\left[\phi_{r b_{*}} \mid \mathcal{R}_{r}, \mathcal{B}_{b_{*}}\right] \\
\Rightarrow \operatorname{Risk}_{2}[\phi \mid \mathcal{R}, \mathcal{B}] & \leq \max _{b \leq B} \sum_{r \leq R} \operatorname{Risk}_{2}\left[\phi_{r b} \mid \mathcal{R}_{r}, \mathcal{B}_{b}\right]
\end{aligned}
$$


Refinement: W.I.o.g. we may assume that the detectors $\phi_{r b}$ are balanced:

$$
\epsilon_{r b}:=\operatorname{Risk}\left[\phi_{r b} \mid \mathcal{R}_{r}, \mathcal{B}_{b}\right]=\operatorname{Risk}_{1}\left[\phi_{r b} \mid \mathcal{R}_{r}, \mathcal{B}_{b}\right]=\operatorname{Risk}_{2}\left[\phi_{r b} \mid \mathcal{R}_{r}, \mathcal{B}_{b}\right] .
$$

Consider matrices

$$
E=\left[\begin{array}{ccc}
\epsilon_{1,1} & \cdots & \epsilon_{1, B} \\
\vdots & \cdots & \vdots \\
\epsilon_{R, 1} & \cdots & \epsilon_{R, B}
\end{array}\right], F=\left[\begin{array}{l|l} 
& E \\
\hline E^{T} &
\end{array}\right]
$$

Note: The maximal eigenvalue $\theta$ of $F$ is the spectral norm $\|E\|_{2,2}$ of $E$, and the leading eigenvector $[g ; f]$ can be selected to be positive (Perron-Frobenius theorem), and we have

$$
\theta g=E f \quad \theta f=E^{T} g
$$

- Let us pass from the detectors $\phi_{r b}$ to shifted detectors $\psi_{r b}=\phi_{r b}-\ln \left(f_{b} / g_{r}\right)$ and assemble the shifted detectors into the detector

$$
\psi(\omega)=\operatorname{maxmin}_{r \leq R} \psi_{r \leq b}(\omega)
$$

By the previous observation

$\operatorname{Risk}_{1}[\psi \mid \mathcal{R}, \mathcal{B}] \leq \max _{r} \sum_{b} \operatorname{Risk}_{1}\left(\psi_{r b} \mid \mathcal{R}_{r}, \mathcal{B}_{b}\right)=\max _{r} \sum_{b} \epsilon_{r b}\left(f_{b} / g_{r}\right)=\max _{r}\left[(E f)_{r} / g_{r}\right]=\theta$ $\operatorname{Risk}_{2}[\psi \mid \mathcal{R}, \mathcal{B}] \leq \max _{b} \sum_{r} \operatorname{Risk}_{2}\left(\psi_{r b} \mid \mathcal{R}_{r}, \mathcal{B}_{b}\right)=\max _{r} \sum_{r} \epsilon_{r b}\left(g_{r} / f_{b}\right)=\max _{b}\left[\left(E^{T} g\right)_{b} / f_{b}\right]=\theta$

$\Rightarrow$ Partial risks of detector $\psi$ on aggregated families $\mathcal{R}, \mathcal{B}$ are $\leq \theta=\|E\|_{2,2}$. 


\section{Detector-Based Tests "Up to Closeness"}

\section{Situation: We are given}

- $L$ families of probability distributions $\mathcal{P}_{\ell}, \ell=1, \ldots, L$, on observation space $\Omega$, giving rise to $L$ hypotheses $H_{\ell}$, on the distribution $P$ of random observation $\omega \in \Omega$ :

$$
H_{\ell}: P \in \mathcal{P}_{\ell}, 1 \leq \ell \leq L
$$

- closeness relation $\mathcal{C}$;

- system of balanced detectors

$$
\left\{\phi_{\ell \ell^{\prime}}: \ell<\ell^{\prime},\left(\ell, \ell^{\prime}\right) \notin \mathcal{C}\right\}
$$

along with upper bounds $\epsilon_{\ell \ell^{\prime}}$ on the detector risks:

$$
\forall\left(\ell, \ell^{\prime}: \ell<\ell^{\prime},\left(\ell, \ell^{\prime}\right) \notin \mathcal{C}\right): \begin{cases}\int_{\Omega} \mathrm{e}^{-\phi_{\ell \ell^{\prime}}(\omega)} P(d \omega) \leq \epsilon_{\ell \ell^{\prime}} & \forall P \in \mathcal{P}_{\ell} \\ \int_{\Omega} \mathrm{e}^{\phi_{\ell \ell^{\prime}}(\omega)} P(d \omega) \leq \epsilon_{\ell \ell^{\prime}} & \forall P \in \mathcal{P}_{\ell^{\prime}}\end{cases}
$$

- Our goal is to build single-observation test deciding on hypotheses $H_{1}, \ldots, H_{L}$ up to closeness $\mathcal{C}$. 


\section{Construction:}

- We set

$$
\phi_{\ell \ell^{\prime}}(\omega)=\left\{\begin{array}{ll}
-\phi_{\ell^{\prime} \ell}(\omega), & \ell>\ell^{\prime},\left(\ell, \ell^{\prime}\right) \notin \mathcal{C}, \\
0, & \left(\ell, \ell^{\prime}\right) \in \mathcal{C},
\end{array} \epsilon_{\ell \ell^{\prime}}= \begin{cases}\epsilon_{\ell^{\prime} \ell}, & \ell>\ell^{\prime},\left(\ell, \ell^{\prime}\right) \notin \mathcal{C}, \\
1, & \left(\ell, \ell^{\prime}\right) \in \mathcal{C},\end{cases}\right.
$$

thus ensuring that

$$
\begin{gathered}
\phi_{\ell \ell^{\prime}}(\cdot)=-\phi_{\ell^{\prime} \ell}(\cdot), \text { and } \epsilon_{\ell \ell^{\prime}}=\epsilon_{\ell^{\prime} \ell}, \quad 1 \leq \ell, \ell^{\prime} \leq L \\
\int_{\Omega} e^{-\phi_{\ell \ell^{\prime}}(\omega)} P(d \omega) \leq \epsilon_{\ell \ell^{\prime}} \quad \forall\left(P \in \mathcal{P}_{\ell}, \quad 1 \leq \ell, \ell^{\prime} \leq L\right)
\end{gathered}
$$

- Given shifts $a_{\ell \ell^{\prime}}=-a_{\ell^{\prime} \ell}$, we specify test $\mathcal{T}$ as follows:

Given observation $\omega, \mathcal{T}$ accepts all hypotheses $H_{\ell}$ such that

$$
\phi_{\ell \ell^{\prime}}(\omega)>a_{\ell \ell^{\prime}} \forall\left(\ell^{\prime}:\left(\ell, \ell^{\prime}\right) \notin \mathcal{C}\right)
$$

and rejects all other hypotheses.

Proposition. The $\mathcal{C}$-risk of $\mathcal{T}$ can be upper-bounded as

$$
\operatorname{Risk}^{\mathcal{C}}\left[\mathcal{T} \mid H_{1}, \ldots, H_{L}\right] \leq \max _{\ell \leq L} \sum_{\ell^{\prime}:\left(\ell, \ell^{\prime}\right) \notin \mathcal{C}} \epsilon_{\ell \ell^{\prime}} \mathrm{e}^{a_{\ell \ell^{\prime}}}
$$


Optimal shifts: Consider the symmetric nonnegative matrix

$$
E=\left[\epsilon_{\ell \ell^{\prime}} \chi_{\left.\ell \ell^{\prime}\right]^{\prime}}\right]_{\ell, \ell^{\prime}=1}^{L}, \quad \chi_{\ell \ell^{\prime}}=\left\{\begin{array}{ll}
1, & \left(\ell, \ell^{\prime}\right) \notin \mathcal{C} \\
0, & \left(\ell, \ell^{\prime}\right) \in \mathcal{C},
\end{array},\right.
$$

and let $\theta=\|E\|_{2,2}$ be the spectral norm of $E$, or, which is the same under the circumstances, the largest eigenvalue of $E$.

By the Perron-Frobenius theorem, for every $\theta^{\prime}>\theta$ there exists a positive vector $f$ such that

$$
E f \leq \theta^{\prime} f
$$

the same holds true for $\theta^{\prime}=\theta$, provided the leading eigenvector of $E$ (which always can selected to be nonnegative) is positive.

Observation: For $\alpha_{\ell \ell^{\prime}}=\ln \left(f_{\ell^{\prime}} / f_{\ell}\right)$, the risk bound from the proposition reads

$$
\operatorname{Risk}^{\mathcal{C}}\left[\mathcal{T} \mid H_{1}, \ldots, H_{L}\right] \leq \theta^{\prime} .
$$

Thus, assembling the detectors $\phi_{\ell \ell^{\prime}}$ appropriately, one can get a test with $\mathcal{C}$ risk arbitrarily close to $\|E\|_{2,2}$. 
Utilizing repeated observations. We can apply the above construction to

- $K$-repeated observation $\omega^{K}=\left(\omega_{1}, \ldots, \omega_{K}\right)$ in the role of $\omega$,

- direct products $\mathcal{P}_{\ell}^{\oplus, K}=\underset{k=1}{\bigoplus} \mathcal{P}_{\ell}$ in the role of families $\mathcal{P}_{\ell}$, and respective hypotheses $H_{\ell}^{\oplus, K}$ in the role of hypotheses $H_{\ell}, \ell=1, \ldots, L$,

- detectors $\phi_{\ell \ell^{\prime}}^{(K)}\left(\omega^{K}\right)=\sum_{k=1}^{K} \phi_{\ell \ell^{\prime}}\left(\omega_{k}\right)$ in the role of detectors $\phi_{\ell \ell^{\prime}}$, which allows to replace $\epsilon_{\ell \ell^{\prime}}$ with $\epsilon_{\ell \ell^{\prime}}^{K}$.

As a result, we get $K$-observation test $\mathcal{T}^{K}$ such that

$$
\operatorname{Risk}^{\mathcal{C}}\left[\mathcal{T}^{K} \mid H_{1}^{\oplus, K}, \ldots, H_{L}^{\oplus, K}\right] \leq \theta_{K}^{\prime}
$$

where $\theta_{K}^{\prime}$ can be made arbitrarily close (under favorable circumstances, even equal) to the quantity

$$
\left\|\left[\epsilon_{\ell \ell^{\prime}}^{K} \chi_{\ell \ell^{\prime}}\right]_{\ell, \ell^{\prime}=1}^{L}\right\|_{2,2}, \chi_{\ell \ell^{\prime}}= \begin{cases}1, & \left(\ell, \ell^{\prime}\right) \notin \mathcal{C} \\ 0, & \left(\ell, \ell^{\prime}\right) \in \mathcal{C}\end{cases}
$$

In particular, in the case when $\epsilon_{\ell \ell^{\prime}}<1$ whenever $\left(\ell, \ell^{\prime}\right) \notin \mathcal{C}$, we can ensure that the $\mathcal{C}$-risk of $\mathcal{T}^{K}$ converges to 0 exponentially fast as $K \rightarrow \infty$. 


\section{"Universality" of detector-based tests.}

Proposition. Let $\mathcal{P}_{\chi}, \chi=1,2$, be two families of probability distributions on observation space $\Omega$, and let $H_{\chi}, \chi=1,2$, be associate hypotheses on the distribution of an observation.

Assume that there exists a simple deterministic or randomized test $\mathcal{T}$ deciding on $H_{1}, H_{2}$ with risk $\leq \epsilon \in(0,1 / 2)$. Then there exists a detector $\phi$ with

$$
\operatorname{Risk}\left[\phi \mid \mathcal{P}_{1}, \mathcal{P}_{2}\right] \leq \epsilon_{+}:=2 \sqrt{\epsilon[1-\epsilon]}<1 .
$$


Proof. $\mathbf{1}^{\circ}$. Indeed, let $\mathcal{T}$ be deterministic, let $\Omega_{\chi}=\{\omega \in \Omega: \mathcal{T}(\omega)=\{\chi\}\}, \chi=1,2$, and let

$$
\phi(\omega)= \begin{cases}\frac{1}{2} \ln \left(\frac{[1-\epsilon]}{\epsilon}\right), & \omega \in \Omega_{1} \\ \left.\frac{1}{2} \ln (\epsilon / 1-\epsilon)\right), & \omega \in \Omega_{2}\end{cases}
$$

Then for $P \in \mathcal{P}_{1}$ and $\epsilon^{\prime}=\int_{\Omega_{2}} P(d \omega)$ we have

$$
\begin{aligned}
\Rightarrow \int \mathrm{e}^{-\phi(\omega)} P(d \omega) & =\sqrt{\epsilon /[1-\epsilon]}\left(1-\epsilon^{\prime}\right)+\sqrt{[1-\epsilon] / \epsilon} \epsilon^{\prime} \\
& =\sqrt{\epsilon /[1-\epsilon]}+\underbrace{[\sqrt{[1-\epsilon] / \epsilon}-\sqrt{\epsilon /[1-\epsilon]}]}_{\geq 0} \underbrace{\epsilon^{\prime}}_{\leq \epsilon} \\
& \leq \sqrt{\epsilon /[1-\epsilon]}+[\sqrt{[1-\epsilon] / \epsilon}-\sqrt{\epsilon /[1-\epsilon]}] \epsilon \\
& =2 \sqrt{\epsilon[1-\epsilon]}
\end{aligned}
$$

In the same way, when $P \in \mathcal{P}_{2}$ and $\epsilon^{\prime}=\int_{\Omega_{1}} P(d \omega)$,

$$
\int \mathrm{e}^{\phi(\omega)} P(d \omega)=\sqrt{\epsilon /[1-\epsilon]}\left(1-\epsilon^{\prime}\right)+\sqrt{[1-\epsilon] / \epsilon} \epsilon^{\prime}=2 \sqrt{\epsilon[1-\epsilon]}
$$

We conclude that

$$
\operatorname{Risk}_{\chi}\left[\phi \mid \mathcal{P}_{1}, \mathcal{P}_{2}\right] \leq 2 \sqrt{\epsilon[1-\epsilon]}
$$


$\mathbf{2}^{\circ}$. Now let $\mathcal{T}$ be randomized. Setting $\mathcal{P}_{\chi}^{+}=\left\{P \times\right.$ Uniform $\left.[0,1]: P \in \mathcal{P}_{\chi}\right\}, \chi=1,2$, $\Omega^{+}=\Omega \times[0,1]$.

By the above there exists a bounded detector $\phi_{+}: \Omega^{+} \rightarrow \mathbb{R}$ such that

$$
\begin{aligned}
& \forall\left(P \in \mathcal{P}_{1}\right): \int_{\Omega}\left[\int_{0}^{1} \mathrm{e}^{-\phi_{+}(\omega, s)} d s\right] P(d \omega) d s \leq \bar{\epsilon}=2 \sqrt{\epsilon[1-\epsilon]}, \\
& \forall\left(P \in \mathcal{P}_{2}\right): \int_{\Omega}\left[\int_{0}^{1} \mathrm{e}^{\phi_{+}(\omega, s)} d s\right] P(d \omega) \leq \bar{\epsilon},
\end{aligned}
$$

whence, setting $\phi(\omega)=\int_{0}^{1} \phi(\omega, s) d s$ and applying Jensen's Inequality,

$$
\forall\left(P \in \mathcal{P}_{1}\right): \int_{\Omega} \mathrm{e}^{-\phi(\omega)} P(d \omega) \leq \bar{\epsilon}, \forall\left(P \in \mathcal{P}_{2}\right): \int_{\Omega} \mathrm{e}^{\phi(\omega)} P(d \omega) \leq \bar{\epsilon}
$$

- Risk $2 \sqrt{\epsilon[1-\epsilon]}$ of the detector-based test induced by simple test $\mathcal{T}$ is "much worse" than the risk $\epsilon$ of $\mathcal{T}$.

- However, when repeated observations are allowed, we can compensate for risk deterioration $\epsilon \rightarrow 2 \sqrt{\epsilon[1-\epsilon]}$ by passing in the detector-based test from a single observation to a moderate number of them. 


\section{Minimal risk detectors}

$$
\inf _{\phi}\left\{\operatorname{Risk}\left[\phi \mid \mathcal{P}_{1}, \mathcal{P}_{2}\right]=\min \left\{\epsilon: \begin{array}{rl}
\int_{\Omega} \mathrm{e}^{-\phi(\omega)} P(d \omega) & \leq \epsilon \forall\left(P \in \mathcal{P}_{1}\right) \\
\int_{\Omega} \mathrm{e}^{\phi(\omega)} P(d \omega) & \leq \epsilon \forall\left(P \in \mathcal{P}_{2}\right)
\end{array}\right\}\right\}
$$

- Optimization problem specifying risk has constraints convex in $(\phi, \epsilon)$

- When passing from families $\mathcal{P}_{\chi}, \chi=1,2$, to their convex hulls, the risk of a detector remains intact.

It would be nice to be able to solve (!), thus arriving at the lowest risk detector-based tests.

However: (!) is an optimization problem with infinite-dimensional decision "vector" and infinitely many constraints $\Rightarrow$ in general, (!) is intractable.

Simple observation schemes: Special cases where (!) is efficiently solvable via Convex Optimization. 


\section{Simple Observation Schemes}

Simple Observation Scheme is a collection

$$
\mathcal{O}=\left((\Omega, \Pi),\left\{p_{\mu}: \mu \in \mathcal{M}\right\}, \mathcal{F}\right),
$$

where

- $(\Omega, \Pi)$ is a (complete separable metric) observation space $\Omega$ with ( $\sigma$-finite $\sigma$-additive) reference measure $\Pi$,

$$
\operatorname{supp} \Pi=\Omega \text {; }
$$

- $\left\{p_{\mu}(\cdot): \mu \in \mathcal{M}\right\}$ is a parametric family of probability densities, taken w.r.t. $\Pi$, on $\Omega$, and

- $\mathcal{M}$ is a relatively open convex set in some $\mathbb{R}^{n}$

- $p_{\mu}(\omega)$ : positive and continuous in $\mu \in \mathcal{M}, \omega \in \Omega$

- $\mathcal{F}$ is a finite-dimensional space of continuous functions on $\Omega$ containing constants and such that

$$
\ln \left(p_{\mu}(\cdot) / p_{\nu}(\cdot)\right) \in \mathcal{F} \quad \forall \mu, \nu \in \mathcal{M} .
$$

- For $\phi \in \mathcal{F}$, the function

is finite and concave in $\mu \in \mathcal{M}$.

$$
\mu \mapsto \ln \left(\int_{\Omega} \mathrm{e}^{\phi(\omega)} p_{\mu}(\omega) \Pi(d \omega)\right)
$$


Example 1: Gaussian o.s., where $(\Omega, \Pi)$ is $\mathbb{R}^{d}$ with Lebesgue measure,

$$
\begin{gathered}
\left\{p_{\mu}(\cdot)=\mathcal{N}\left(\mu, I_{d}\right): \mu \in \mathbb{R}^{d}\right\}, \mathcal{F}=\{\text { affine functions on } \Omega\} \\
\Rightarrow\left\{\begin{array}{l}
\ln \left(p_{\mu}(\cdot) / p_{\nu}(\cdot)\right) \in \mathcal{F}, \\
\ln \left(\int_{\Omega} \mathrm{e}^{a^{T} \omega+b} p_{\mu}(\omega) \Pi(d \omega)\right)=a^{T} \mu+b+\frac{a^{T} a}{2} \text { is concave in } \mu .
\end{array}\right.
\end{gathered}
$$

- Gaussian o.s. is the standard observation model in Signal Processing.

Example 2: Discrete o.s., where $(\Omega, \Pi)$ is finite set $\{1, \ldots, d\}$ with counting measure,

$$
\left\{p_{\mu}(\omega)=\mu_{\omega}, \mu \in \mathcal{M}=\left\{\mu>0: \sum_{\omega=1}^{d} \mu_{\omega}=1\right\}\right\}
$$

is the set of non-vanishing probability distributions on $\Omega$, and $\mathcal{F}=$ all functions on $\Omega$ \}

$\Rightarrow\left\{\begin{array}{l}\ln \left(p_{\mu}(\cdot) / p_{\nu}(\cdot)\right) \in \mathcal{F}, \\ \ln \left(\int_{\Omega} \mathrm{e}^{\phi(\omega)} p_{\mu}(\omega) \Pi(d \omega)\right)=\ln \left(\sum_{\omega \in \Omega} \mathrm{e}^{\phi(\omega)} \mu_{\omega}\right) \text { is concave in } \mu .\end{array}\right.$ 
Example 3: Poisson o.s., where $(\Omega, \Pi)$, is the nonnegative part $\mathbf{Z}_{+}^{d}$ of integer lattice in $\mathbb{R}^{d}$ equipped with counting measure,

$$
\left\{p_{\mu}(\omega)=\prod_{i=1}^{d} \frac{\mu_{i}^{\omega_{i}} \mathrm{e}^{-\mu_{i}}}{\omega_{i} !}: \mu \in \mathcal{M}:=\mathbb{R}_{++}^{d}\right\}
$$

is the family of distributions of random vectors with independent across $i$ Poisson entries $\omega_{i} \sim$ Poisson $\left(\mu_{i}\right)$, and $\mathcal{F}=\{$ affine functions on $\Omega$ \}

$\Rightarrow\left\{\begin{array}{l}\ln \left(p_{\mu}(\cdot) / p_{\nu}(\cdot)\right) \in \mathcal{F}, \\ \ln \left(\int_{\Omega} \mathrm{e}^{a^{T} \omega+b} p_{\mu}(\omega) \Pi(d \omega)\right)=\sum_{i}\left(\mathrm{e}^{a_{i}}-1\right) \mu_{i} \text { is concave in } \mu .\end{array}\right.$

- Poisson o.s. arises in Poisson Imaging, including

- Positron Emission Tomography,

- Large binocular Telescope,

- Nanoscale Fluorescent Microscopy. 


\section{Example 4: Direct product of simple o.s.'s.}

Simple o.s.'s

$$
\mathcal{O}_{k}=\left(\left(\Omega_{k}, \Pi_{k}\right),\left\{p_{\mu_{k}, k}(\cdot): \mu_{k} \in \mathcal{M}_{k}\right\}, \mathcal{F}_{k}\right), 1 \leq k \leq K
$$

give rise to their direct product $\otimes_{k=1}^{K} \mathcal{O}_{k}$ defined as the o.s.

$$
\left(\left(\Omega^{K}, \Pi^{K}\right),\left\{p_{\mu^{K}}(\cdot): \mu^{K} \in \mathcal{M}^{K}\right\}, \mathcal{F}^{K}\right)
$$

where

- $\Omega^{K}=\Omega_{1} \times, \ldots \times \Omega_{K}, \Pi^{K}=\Pi_{1} \times \ldots \times \Pi_{K}$

- $\mathcal{M}^{K}=\mathcal{M}_{1} \times \ldots \times \mathcal{M}_{K}, p_{\left(\mu_{1}, \ldots, \mu_{K}\right)}\left(\omega_{1}, \ldots, \omega_{K}\right)=\prod_{k=1}^{K} p_{\mu_{k}, k}\left(\omega_{k}\right)$

- $\mathcal{F}^{K}=\left\{\phi(\underbrace{\omega_{1}, \ldots, \omega_{K}}_{\omega^{K}})=\sum_{k=1}^{K} \phi_{k}\left(\omega_{k}\right): \phi_{k} \in \mathcal{F}_{k}, 1 \leq k \leq K\right\}$

- A direct product of simple o.s.'s is a simple o.s. 


\section{Example 5: Power of a simple o.s.}

When all $K$ o.s.'s in product $\mathcal{O}^{K}=\bigotimes_{k=1}^{K} \mathcal{O}_{k}$ are identical to each other:

$$
\mathcal{O}_{k}=\mathcal{O}:=\left((\Omega, \Pi),\left\{p_{\mu}(\cdot): \mu \in \mathcal{M}\right\}, \mathcal{F}\right), 1 \leq k \leq K,
$$

we can "restrict $\mathcal{O}^{K}$ to its diagonal," arriving at $K$-th power $\mathcal{O}^{(K)}$ of $\mathcal{O}$ :

$$
\begin{aligned}
\mathcal{O}^{(K)} & =\left(\left(\Omega^{K}, \Pi^{K}\right),\left\{p_{\mu}^{(K)}(\cdot): \mu \in \mathcal{M}\right\}, \mathcal{F}^{(K)}\right), \\
p_{\mu}^{(K)}\left(\omega_{1}, \ldots, \omega_{K}\right) & =\prod_{k=1}^{K} p_{\mu}\left(\omega_{k}\right), \\
\mathcal{F}^{(K)} & =\left\{\phi^{(K)}\left(\omega^{K}\right)=\sum_{k=1}^{K} \phi\left(\omega_{k}\right): \phi \in \mathcal{F}\right\}
\end{aligned}
$$

- K-th power of a simple o.s. is a simple o.s. 


$$
\varepsilon_{*}\left(\mathcal{P}_{1}, \mathcal{P}_{2}\right)=\min _{\phi(\cdot), \epsilon}\left\{\epsilon: \begin{array}{l}
\int_{\Omega} \mathrm{e}^{-\phi(\omega)} P(d \omega) \leq \epsilon \forall\left(P \in \mathcal{P}_{1}\right) \\
\int_{\Omega} \mathrm{e}^{\phi(\omega)} P(d \omega) \leq \epsilon \forall\left(P \in \mathcal{P}_{2}\right)
\end{array}\right\}
$$

Main Result. Let $\mathcal{O}=\left((\Omega, \Pi),\left\{p_{\mu}(\cdot): \mu \in \mathcal{M}\right\}, \mathcal{F}\right)$ be a simple o.s., and let $M_{1}, M_{2}$ be two nonempty compact convex subsets of $\mathcal{M}$. These subsets give rise to two families of probability distributions $\mathcal{P}_{1}, \mathcal{P}_{2}$ on $\Omega$

$$
\mathcal{P}_{\chi}=\left\{P: \text { the density of } P \text { is } p_{\mu} \text { with } \mu \in \mathcal{M}_{\chi}\right\}, \quad \chi=1,2,
$$

and two hypotheses on the distribution $P$ of random observation $\omega \in \Omega$ :

$$
H_{1}: P \in \mathcal{P}_{1} \text { and } H_{2}: P \in \mathcal{P}_{2}
$$

Consider the function

$$
\begin{array}{r}
\Phi(\phi ; \mu, \nu)=\frac{1}{2}\left[\ln \left(\int_{\Omega} \mathrm{e}^{-\phi(\omega)} p_{\mu}(\omega) \Pi(d \omega)\right)+\ln \left(\int_{\Omega} \mathrm{e}^{\phi(\omega)} p_{\nu}(\omega) \Pi(d \omega)\right)\right]: \\
\mathcal{F} \times\left[M_{1} \times M_{2}\right] \rightarrow \mathbb{R} .
\end{array}
$$


Then

- $\Phi(\phi ; \mu, \nu)$ is continuous on its domain, convex in $\phi \in \mathcal{F}$, concave in $(\mu, \nu) \in \mathcal{M}_{1} \times \mathcal{M}_{2}$ and possesses saddle point (min in $\phi$, $\max$ in $(\mu, \nu)$ ):

there exist $\left(\phi_{*} \in \mathcal{F},\left(\mu^{*}, \nu^{*}\right) \in M_{1} \times M_{2}\right)$ such that

$$
\Phi\left(\phi ; \mu^{*}, \nu^{*}\right) \geq \underbrace{\Phi\left(\phi_{*} ; \mu^{*}, \nu^{*}\right)}_{=\text {SadVal }} \geq \Phi\left(\phi_{*} ; \mu, \nu\right)
$$

for all $\left(\phi \in \mathcal{F},(\mu, \nu) \in M_{1} \times M_{2}\right)$.

- The component $\phi_{*}$ of a saddle point $\left(\phi_{*},\left(\mu^{*}, \nu^{*}\right)\right)$ of $\Phi$ is an optimal solution to (!), and

$$
\varepsilon_{*}\left(\mathcal{P}_{1}, \mathcal{P}_{2}\right)=\exp \left\{\Phi\left(\phi_{*} ; \mu^{*}, \nu^{*}\right)\right\} .
$$


- A saddle point $\left(\phi_{*},\left(\mu^{*}, \nu^{*}\right)\right)$ can be found as follows. We solve the optimization problem

$$
\text { SadVal }=\max _{\mu \in M_{1}, \nu \in M_{2}} \ln \left(\int_{\Omega} \sqrt{p_{\mu}(\omega) p_{\nu}(\omega)} \sqcap(d \omega)\right)
$$

(which is a solvable convex optimization problem), and take an optimal solution to the problem as $\left(\mu^{*}, \nu^{*}\right)$. We then set

$$
\phi_{*}(\omega)=\frac{1}{2} \ln \left(p_{\mu^{*}}(\omega) / p_{\nu^{*}}(\omega)\right),
$$

thus getting an optimal detector $\phi_{*} \in \mathcal{F}$.

- For this detector and the associated simple test $\mathcal{T}_{\phi_{*}}$,

$$
\begin{aligned}
\operatorname{Risk}\left[\mathcal{T}_{\phi_{*}} \mid H_{1}, H_{2}\right] & \leq \operatorname{Risk}\left[\phi_{*} \mid \mathcal{P}_{1}, \mathcal{P}_{2}\right] \\
& \left.=\operatorname{Risk} 1 \phi_{*} \mid \mathcal{P}_{1}, \mathcal{P}_{2}\right]=\operatorname{Risk}_{2}\left[\phi_{*} \mid \mathcal{P}_{1}, \mathcal{P}_{2}\right] \\
& =\varepsilon_{*}\left(\mathcal{P}_{1}, \mathcal{P}_{2}\right)=\mathrm{e}^{\operatorname{SadVal}}=\int_{\Omega} \sqrt{p_{\mu^{*}}(\omega) p_{\nu^{*}}(\omega)} \Pi(d \omega) .
\end{aligned}
$$




$$
\mathrm{SadVal}=\max _{\mu \in M_{1}, \nu \in M_{2}} \ln \left(\int_{\Omega} \sqrt{p_{\mu}(\omega) p_{\nu}(\omega)} \sqcap(d \omega)\right)
$$

Gaussian o.s. $\mathcal{P}_{\chi}=\left\{\mathcal{N}\left(\mu, I_{d}\right): \mu \in M_{\chi}\right\}, \chi=1,2$ :

- Optimization problem (!) reads

$$
\max _{\mu \in M_{1}, \nu \in M_{2}}\left[-\frac{1}{8}\|\mu-\nu\|_{2}^{2}\right]
$$

- The optimal balanced detector and its risk are given by

$$
\mid \begin{gathered}
\phi_{*}(\omega)=\frac{1}{2}\left[\mu^{*}-\nu^{*}\right] \omega-c, \\
{\left[\left(\mu^{*}, \nu^{*}\right) \in \underset{\mu \in M_{1}, \nu \in M_{2}}{\operatorname{Argmin}}\|\mu-\nu\|_{2}^{2}, \quad c=\frac{1}{4}\left[\mu^{*}-\nu^{*}\right]^{T}\left[\mu^{*}+\nu^{*}\right]\right]} \\
\varepsilon_{*}\left(\mathcal{P}_{1}, \mathcal{P}_{2}\right)=\exp \left\{-\frac{\left\|\mu^{*}-\nu^{*}\right\|_{2}^{2}}{8}\right\}
\end{gathered}
$$

Note: We are in the "signal plus noise" model of observations with noise $\sim$ $\mathcal{N}\left(0, I_{d}\right)$. The test $\mathcal{T}_{\phi_{*}}$ is nothing but the pairwise Euclidean separation test associated with $X_{\chi}=M_{\chi}, \chi=1,2$. 


$$
\mathrm{SadVal}=\max _{\mu \in M_{1}, \nu \in M_{2}} \ln \left(\int_{\Omega} \sqrt{p_{\mu}(\omega) p_{\nu}(\omega)} \Pi(d \omega)\right)
$$

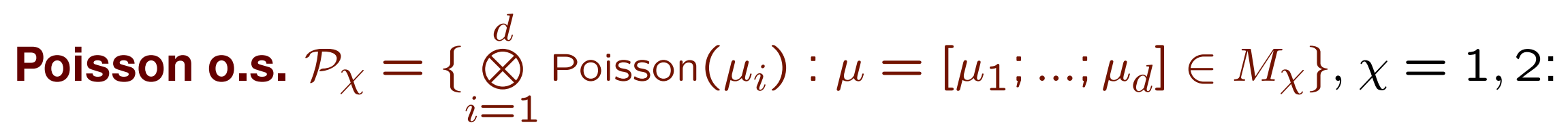

- Problem (!) reads

$$
\max _{\mu \in M_{1}, \nu \in M_{2}} \underbrace{\left[-\frac{1}{2} \sum_{i=1}^{d}\left(\sqrt{\mu_{i}}-\sqrt{\nu_{i}}\right)^{2}\right]}_{\sum_{i}\left[\sqrt{\mu_{i} \nu_{i}}-\frac{1}{2} \mu_{i}-\frac{1}{2} \nu_{i}\right]}
$$

- The optimal balanced detector and its risk are given by

$$
\begin{gathered}
\phi_{*}(\omega)=\frac{1}{2} \sum_{i=1}^{d}\left[\ln \left(\mu_{i}^{*} / \nu_{i}^{*}\right) \omega_{i}+\nu_{i}^{*}-\mu_{i}^{*}\right], \\
{\left[\left(\mu^{*}, \nu^{*}\right) \in \underset{\mu \in M_{1}, \nu \in M_{2}}{\operatorname{Argmax}} \sum_{i}\left[\sqrt{\mu_{i} \nu_{i}}-\frac{1}{2} \mu_{i}-\frac{1}{2} \nu_{i}\right]\right]} \\
\varepsilon_{*}\left(\mathcal{P}_{1}, \mathcal{P}_{2}\right)=\exp \left\{-\frac{1}{2} \sum_{i}\left(\sqrt{\mu_{i}^{*}}-\sqrt{\nu_{i}^{*}}\right)^{2}\right\}
\end{gathered}
$$




$$
\mathrm{SadVal}=\max _{\mu \in M_{1}, \nu \in M_{2}} \ln \left(\int_{\Omega} \sqrt{p_{\mu}(\omega) p_{\nu}(\omega)} \Pi(d \omega)\right)
$$

\section{Discrete 0.s.}

$$
\mathcal{P}_{\chi}=\left\{\mu \in M_{\chi}\right\}, M_{\chi} \subset \Delta_{d}^{o}=\left\{\mu \in \mathbb{R}_{+}^{d}: \sum_{\omega} \mu_{\omega}=1, \mu>0\right\}, \chi=1,2
$$

- Problem (!) reads

$$
\max _{\mu \in M_{1}, \nu \in M_{2}} \sum_{\omega} \sqrt{\mu_{\omega} \nu_{\omega}}
$$

- The optimal balanced detector and its risk are given by

$$
\begin{gathered}
\phi_{*}(\omega)=\frac{1}{2} \ln \left(\mu_{\omega}^{*} / \nu_{\omega}^{*}\right), \omega \in \Omega=\{1, \ldots, d\} \\
{\left[\left(\mu^{*}, \nu^{*}\right) \in \underset{\mu \in M_{1}, \nu \in M_{2}}{\operatorname{Argmin}} \sum_{\omega} \sqrt{\mu_{\omega} \nu_{\omega}}\right]} \\
\varepsilon_{*}\left(\mathcal{P}_{1}, \mathcal{P}_{2}\right)=\sum_{\omega} \sqrt{\mu_{\omega}^{*} \nu_{\omega}^{*}}
\end{gathered}
$$


Direct product of simple o.s.'s. Let

$$
\mathcal{O}_{k}=\left(\left(\Omega_{k}, \Pi_{k}\right),\left\{p_{\mu_{k}, k}(\cdot): \mu_{k} \in \mathcal{M}_{k}\right\}, \mathcal{F}_{k}\right), 1 \leq k \leq K,
$$

be simple o.s.'s, and $M_{\chi, k} \subset \mathcal{M}_{k}, \chi=1,2$, be nonempty convex compact sets. Consider the simple o.s.

$$
\left(\left(\Omega^{K}, \Pi^{K}\right),\left\{p_{\mu^{K}}: \mu^{K} \in \mathcal{M}^{K}\right\}, \mathcal{F}^{K}\right)=\bigotimes_{k=1}^{K} \mathcal{O}_{k}
$$

along with two compact convex sets

$$
M_{\chi}=M_{\chi, 1} \times \ldots \times M_{\chi, K}, \chi=1,2 .
$$

Question: What is the problem

$$
\max _{\mu^{K} \in M_{1}, \nu^{K} \in M_{2}} \ln \left(\int_{\Omega^{K}} \sqrt{p_{\mu^{K}}\left(\omega^{K}\right) p_{\nu^{K}}\left(\omega^{K}\right)} \Pi^{K}\left(d \omega^{K}\right)\right)
$$

responsible for the smallest risk detector for the families of distributions $\mathcal{P}_{\chi}^{K}$, $\mathcal{P}_{2}^{K}$ associated in $\mathcal{O}^{K}$ with the sets $M_{\chi}, \chi=1,2$ ? 
Answer: This is the separable problem

$$
\max _{\left\{\mu_{k} \in M_{1, k}, \nu_{k} \in M_{2, k}\right\}_{k=1}^{K}} \sum_{k=1}^{K} \ln \left(\int_{\Omega_{k}} \sqrt{p_{\mu_{k}, k}\left(\omega_{k}\right) p_{\nu_{k}, k}\left(\omega_{k}\right)} \Pi_{k}\left(d \omega_{k}\right)\right)
$$

$\Rightarrow$ Minimum risk balanced detector for $\mathcal{P}_{1}^{K}, \mathcal{P}_{2}^{K}$ can be chosen as

$$
\begin{gathered}
\phi_{*}^{K}\left(\omega_{1}, \ldots, \omega_{K}\right)=\sum_{k=1}^{K} \phi_{*, k}\left(\omega_{k}\right), \\
\phi_{*, k}\left(\omega_{k}\right)=\frac{1}{2} \ln \left(p_{\mu_{k}^{*}, k}(\omega) / p_{\nu_{k}^{*}, k}(\omega)\right) \\
{\left[\left(\mu_{k}^{*}, \nu_{k}^{*}\right) \in \underset{\mu_{k} \in M_{1, k}, \nu_{k} \in M_{2, k}}{\operatorname{Argmax}} \ln \left(\int_{\Omega_{k}} \sqrt{p_{\mu_{k}, k}\left(\omega_{k}\right) p_{\nu_{k}, k}\left(\omega_{k}\right)} \Pi_{k}\left(d \omega_{k}\right)\right)\right]}
\end{gathered}
$$

and

$$
\varepsilon_{*}\left(\mathcal{P}_{1}^{K}, \mathcal{P}_{2}^{K}\right)=\prod_{k=1}^{K} \varepsilon_{*}\left(\mathcal{P}_{1, k}, \mathcal{P}_{2, k}\right),
$$

where $\mathcal{P}_{\chi, k}$ are the families of distributions associated in $\mathcal{O}_{k}$ with $M_{\chi, k}, \chi=$ $1,2$. 
Power of a simple o.s. Let

$$
\mathcal{O}=\left((\Omega, \Pi),\left\{p_{\mu}(\cdot): \mu \in \mathcal{M}\right\}, \mathcal{F}\right)
$$

be a simple o.s., and $M_{\chi} \subset \mathcal{M}, \chi=1,2$, be nonempty convex compact sets. Consider the $K$-th power of $\mathcal{O}$, that is, the simple o.s.

$$
\mathcal{O}^{(K)}=\left(\left(\Omega^{K}, \Pi^{K}\right),\left\{p_{\mu}^{(K)}\left(\omega_{1}, \ldots, \omega_{K}\right)=\prod_{k=1}^{K} p_{\mu}\left(\omega_{k}\right): \mu \in \mathcal{M}\right\}, \mathcal{F}^{(K)}\right) \text {. }
$$

Question: What is the problem

$$
\max _{\mu \in M_{1}, \nu \in M_{2}} \ln \left(\int_{\Omega^{K}} \sqrt{p_{\mu}^{(K)}\left(\omega^{K}\right) p_{\nu}^{(K)}\left(\omega^{K}\right)} \Pi^{K}\left(d \omega^{K}\right)\right)
$$

responsible for the smallest risk detector for the families of distributions $\mathcal{P}_{\chi}^{(K)}$ associated in $\mathcal{O}^{(K)}$ with the sets $M_{\chi}, \chi=1,2$ ? 
Answer: This is the separable problem

$$
\max _{\left\{\mu \in M_{1}, \nu \in M_{2}\right\}_{k=1}^{K}} \underbrace{\sum_{k=1}^{K} \ln \left(\int_{\Omega} \sqrt{p_{\mu}\left(\omega_{k}\right) p_{\nu}\left(\omega_{k}\right)} \Pi\left(d \omega_{k}\right)\right)}_{K \ln \left(\int_{\Omega} \sqrt{p_{\mu}(\omega) p_{\nu}(\omega)} \Pi(d \omega)\right)}
$$

$\Rightarrow$ Minimum risk balanced detector for $\mathcal{P}_{1}^{(K)}, \mathcal{P}_{2}^{(K)}$ can be chosen as

$$
\begin{gathered}
\phi_{*}^{(K)}\left(\omega_{1}, \ldots, \omega_{K}\right)=\sum_{k=1}^{K} \phi_{*}\left(\omega_{k}\right), \\
\phi_{*}\left(\omega_{k}\right)=\frac{1}{2} \ln \left(p_{\mu^{*}}(\omega) / p_{\nu^{*}}(\omega)\right) \\
{\left[\left(\mu^{*}, \nu^{*}\right) \in \underset{\mu \in M_{1}, \nu \in M_{2}}{\operatorname{Argmax}} \ln \left(\int_{\Omega} \sqrt{p_{\mu}(\omega) p_{\nu}(\omega)} \sqcap(d \omega)\right)\right]}
\end{gathered}
$$

and

$$
\varepsilon_{*}\left(\mathcal{P}_{1}^{(K)}, \mathcal{P}_{2}^{(K)}\right)=\left[\varepsilon_{*}\left(\mathcal{P}_{1}, \mathcal{P}_{2}\right)\right]^{K},
$$

where $\mathcal{P}_{\chi}$ are the families of distributions associated in $\mathcal{O}$ with $M_{\chi}, \chi=1,2$. 


\section{Near-Optimality of Detector-Based Tests in Simple O.S.}

Proposition A. Let

$$
\mathcal{O}=\left((\Omega, \Pi),\left\{p_{\mu}: \mu \in \mathcal{M}\right\}, \mathcal{F}\right)
$$

be a simple o.s., $M_{\chi} \subset \mathcal{M}, \chi=1,2$, be nonempty convex compact sets, giving rise to families of distributions

$$
\mathcal{P}_{\chi}=\left\{P: P \text { has density } p_{\mu}(\cdot) \text { w.r.t. } \Pi \text { with } \mu \in M_{\chi}\right\}, \chi=1,2 \text {, }
$$

hypotheses $H_{\chi}: P \in \mathcal{P}_{\chi}, \chi=1,2$, on the distribution of a random observation $\omega \in \Omega$, and minimum risk detector $\phi_{*}$ for $\mathcal{P}_{1}, \mathcal{P}_{2}$.

Assume that in the nature there exists a simple single-observation test, deterministic or randomized, $\mathcal{T}$ with

$$
\operatorname{Risk}\left[\mathcal{T} \mid H_{1}, H_{2}\right] \leq \epsilon<1 / 2 .
$$

Then the risk of the simple test $\mathcal{T}_{\phi_{*}}$ accepting $H_{1}$ when $\phi_{*}(\omega) \geq 0$ and accepting $\mathrm{H}_{2}$ otherwise "is comparable" to $\epsilon$ :

$$
\operatorname{Risk}\left[\mathcal{T}_{\phi_{*}} \mid H_{1}, H_{2}\right] \leq \bar{\epsilon}:=2 \sqrt{\epsilon(1-\epsilon)}<1 .
$$


Proof. From what we called "universality" of detector-based tests, there exists a detector $\phi$ with $\operatorname{Risk}\left[\phi \mid \mathcal{P}_{1}, \mathcal{P}_{2}\right] \leq \bar{\epsilon}$, and $\operatorname{Risk}\left[\phi_{*} \mid \mathcal{P}_{1}, \mathcal{P}_{2}\right]$ can be only less than $\operatorname{Risk}\left[\phi \mid \mathcal{P}_{1}, \mathcal{P}_{2}\right]$. 
Proposition B. Let $\mathcal{O}=\left((\Omega, \Pi),\left\{p_{\mu}: \mu \in \mathcal{M}\right\}, \mathcal{F}\right)$ be a simple o.s., and $M_{\chi} \subset \mathcal{M}, \chi=$ 1,2 , be nonempty convex compact sets, giving rise to families of distributions

$$
\mathcal{P}_{\chi}=\left\{P: P \text { has density } p_{\mu}(\cdot) \text { w.r.t. } \Pi \text { with } \mu \in M_{\chi}\right\}, \chi=1,2
$$

their powers

$$
\mathcal{P}_{\chi}^{K}=\left\{P \times \ldots \times P: P \in \mathcal{P}_{\chi}\right\}, \chi=1,2, K=1,2, \ldots
$$

hypotheses

$$
H_{\chi}^{K}: P \in \mathcal{P}_{\chi}^{K}, \chi=1,2, K=1,2, \ldots
$$

on the distribution of a random $K$-repeated observation $\omega^{K}=\left(\omega_{1}, \ldots \omega_{K}\right) \in \Omega^{K}$, and minimum risk detector $\phi_{*}$ for $\mathcal{P}_{1}, \mathcal{P}_{2}$.

Assume that "in the nature" there exists positive integer $K_{*}$ and a simple $K_{*}$-observation test, deterministic or randomized, $\mathcal{T}_{K_{*}}$ capable to decide on the hypotheses $H_{\chi}^{K_{*}}, \chi=1,2$, with risk $\leq \epsilon<1 / 2$. Then the test $\mathcal{T}_{\phi_{*}, K}$ deciding on $H_{\chi}^{K}, \chi=1,2$, by accepting $H_{1}^{K}$ whenever

$$
\phi^{(K)}\left(\omega^{K}\right):=\sum_{k=1}^{K} \phi_{*}\left(\omega_{k}\right) \geq 0
$$

and accepting $H_{2}^{K}$ otherwise, satisfies

$$
\operatorname{Risk}\left[\mathcal{T}_{\phi_{*}} \mid H_{1}^{K}, H_{2}^{K}\right] \leq \epsilon \forall K \geq \widehat{K}_{*}=\frac{2}{1-\frac{\ln (4(1-\epsilon))}{\ln (1 / \epsilon)}} K_{*} .\left(\widehat{K}_{*} / K_{*} \rightarrow 2 \text { as } \epsilon \rightarrow+0\right) .
$$

Moreover, this risk bound remains true when the hypotheses $H_{\chi}^{K}$ are extended to hypotheses $H_{\chi}^{\oplus, K}$ stating that the distribution $P$ of $\omega^{K}$ belongs to the quasi direct $K$-th power of $\mathcal{P}_{\chi}$, $\chi=1,2$. 
Proof. As we know, $K_{*}$-th power $\mathcal{O}^{(K)}$ of $\mathcal{O}$ is simple o.s. along with $\mathcal{O}$, and $\phi_{*}^{(K)}$ is the minimum risk detector for the families $\mathcal{P}_{\chi}^{K *}, \chi=1,2$, the risk of this detector being $\left[\varepsilon_{*}\left(\mathcal{P}_{1}, \mathcal{P}_{2}\right)\right]^{K}$. By Proposition $A$ as applied to $\mathcal{O}^{(K)}$ in the role of $\mathcal{O}$, we have

$$
\left[\varepsilon_{*}\left(\mathcal{P}_{1}, \mathcal{P}_{2}\right)\right]^{K_{*}} \leq 2 \sqrt{\epsilon(1-\epsilon)} \Rightarrow \varepsilon_{*}\left(\mathcal{P}_{1}, \mathcal{P}_{2}\right) \leq[2 \sqrt{\epsilon(1-\epsilon)}]^{1 / K_{*}}<1 .
$$

By Detector Calculus, it follows that for $K=1,2, \ldots$ it holds

$$
\operatorname{Risk}\left[\phi_{*}^{(K)} \mid \mathcal{P}_{1}^{\oplus, K}, \mathcal{P}_{2}^{\oplus, K}\right] \leq[2 \sqrt{\epsilon(1-\epsilon)}]^{K / K_{*}}
$$

and the right hand side is $\leq \epsilon$ whenever $K \geq \widehat{K}_{*}$. 


\section{Near-Optimality of Detector-Based Up to Closeness Testing}

Situation: We are given a simple o.s.

$$
\mathcal{O}=\left((\Omega, \Pi),\left\{p_{\mu}: \mu \in \mathcal{M}\right\}, \mathcal{F}\right)
$$

and a collection of nonempty convex compact subsets $M_{\ell}, 1 \leq \ell \leq L$ giving rise to

- Families

$$
\mathcal{P}_{\ell}=\left\{P: P \text { admits density } p_{\mu}, \mu \in M_{\ell} \text { w.r.t. } \Pi\right\}, \ell=1, \ldots, L,
$$

along with direct products $\mathcal{P}_{\ell}^{\oplus, K}=\oplus_{k=1}^{K} \mathcal{P}_{\ell}$ of $\mathcal{P}_{\ell}$ and hypotheses $H_{\ell}^{\oplus, K}$ : $P^{K} \in \mathcal{P}_{\ell}^{\oplus, K}$ on the distribution of $K$-repeated observation $\omega^{K}=\left(\omega_{1}, \ldots, \omega_{K}\right)$, - minimum-risk balanced single-observation detectors $\phi_{\ell \ell^{\prime}}(\omega)$ for $\mathcal{P}_{\ell}, \mathcal{P}_{\ell^{\prime}}$ along with their risks $\varepsilon_{*}\left(\mathcal{P}_{\ell}, \mathcal{P}_{\ell^{\prime}}\right), 1 \leq \ell<\ell^{\prime} \leq L$, and $K$-repeated versions

$$
\phi_{\ell \ell^{\prime}}^{K}\left(\omega^{K}\right)=\sum_{k=1}^{K} \phi_{\ell \ell^{\prime}}\left(\omega_{k}\right)
$$

of $\phi_{\ell \ell^{\prime}}$ such that $\operatorname{Risk}\left[\phi_{\ell \ell^{\prime}}^{(K)} \mid H_{\ell}^{\oplus, K}, H_{\ell^{\prime}}^{\oplus, K}\right] \leq\left[\varepsilon_{*}\left(\mathcal{P}_{\ell}, \mathcal{P}_{\ell^{\prime}}\right)\right]^{K}$. 
- Assume that in addition to the above data, we are given a closeness relation $\mathcal{C}$ on $\{1, \ldots, L\}$. Applying Calculus of Detectors, for every positive integer $K$, setting

$$
\theta_{K}=\|\left[\varepsilon_{*}{ }^{K}\left(\mathcal{P}_{\ell}, \mathcal{P}_{\ell^{\prime}}\right) \cdot\left\{\begin{array}{ll}
1, & \left(\ell, \ell^{\prime}\right) \notin \mathcal{C} \\
0, & \left(\ell, \ell^{\prime}\right) \in \mathcal{C}
\end{array}\right]_{\ell, \ell^{\prime}=1}^{L} \|_{2,2}\right.
$$

we can assemble the outlined data, in a computationally efficient fashion, into a $K$-observation test $\mathcal{T}^{K}$ deciding on $H_{\ell}^{\oplus, K}, 1 \leq \ell \leq L$, with $\mathcal{C}$-risk upperbounded as follows:

$$
\operatorname{Risk}^{\mathcal{C}}\left[\mathcal{T}^{K} \mid H_{1}^{\oplus, K}, \ldots, H_{L}^{\oplus, K}\right] \leq \varkappa \theta_{K}
$$

( $\varkappa>1$ can be selected to be as close to 1 as we want). 
Proposition. In the just described situation, assume that for some $\epsilon<1 / 2$ and $K_{*}$ in the nature there exists a $K_{*}$-observation test $\mathcal{T}$, deterministic or randomized, deciding on the hypotheses

$$
H_{\ell}^{K_{*}}: \omega^{K_{*}}=\left(\omega_{1}, \ldots, \omega_{K_{*}}\right) \text { is an i.i.d. sample drawn from a } P \in \mathcal{P}_{\ell} \text {, }
$$

$\ell=1, \ldots, L$, with $\mathcal{C}$-risk $\leq \epsilon$. Then the test $\mathcal{T}^{K}$ with

$$
K \geq 2 \underbrace{\left[\frac{1+\ln (\varkappa L) / \ln (1 / \epsilon)}{1-\ln (4(1-\epsilon)) / \ln (1 / \epsilon)}\right]}_{\rightarrow 1 \text { as } \epsilon \rightarrow+0} K_{*}
$$

decides on $H_{\ell}^{\oplus, K}, \ell=1, \ldots, L$, with $\mathcal{C}$-risk $\leq \epsilon$ as well. 
Proof. $1^{\circ}$ Let us fix $\ell, \ell^{\prime}$ such that $\left(\ell, \ell^{\prime}\right) \notin \mathcal{C}$, and let us convert $\mathcal{T}$ into a simple $K_{*}$-observation test $\widetilde{\mathcal{T}}_{\ell, \ell^{\prime}}$ deciding on $H_{\ell}^{K_{*}}, H_{\ell^{\prime}}^{K_{*}}$ as follows: whenever $\ell \in \mathcal{T}\left(\omega^{K}\right), \widetilde{\mathcal{T}}_{\ell, \ell^{\prime}}$ accepts $H_{\ell}^{K_{*}}$ and rejects $H_{\ell^{\prime}}^{K_{*}}$, otherwise the test accepts $H_{\ell^{\prime}}^{K_{*}}$ and rejects $H_{\ell}^{K_{*}}$. It is immediately seen that

$$
\operatorname{Risk}\left[\tilde{\mathcal{T}}_{\ell, \ell^{\prime}} \mid H_{\ell}^{K_{*}}, H_{\ell^{\prime}}^{K_{*}}\right] \leq \epsilon .
$$

Indeed, let $P^{K_{*}}=P \times \ldots \times P$ be the distribution of $\omega^{K_{*}}$. Whenever $P^{K}$ obeys

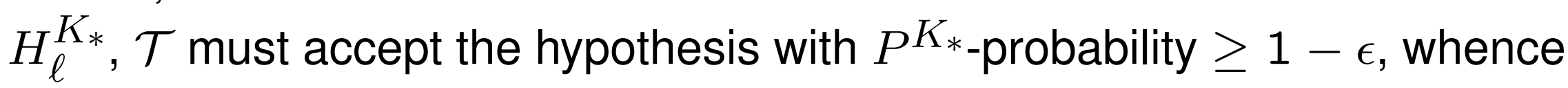

$$
\operatorname{Risk}_{1}\left[\widetilde{\mathcal{T}}_{\ell, \ell^{\prime}} \mid H_{\ell}^{K_{*}}, H_{\ell^{\prime}}^{K_{*}}\right] \leq \epsilon .
$$

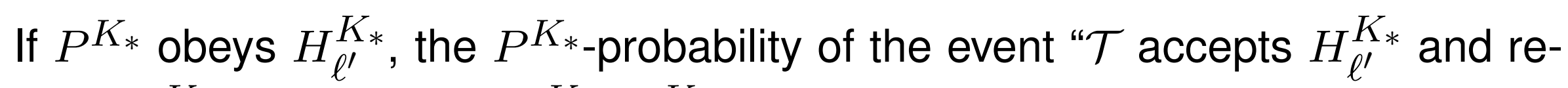

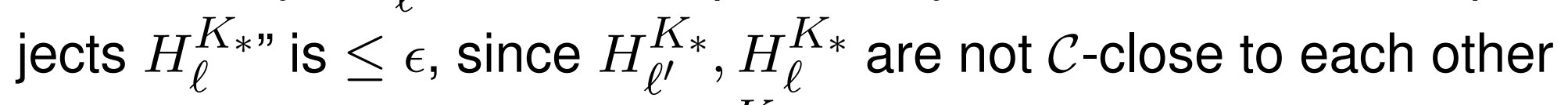

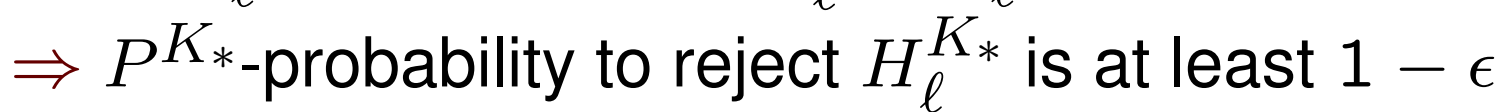

$\Rightarrow \operatorname{Risk}_{2}\left[\widetilde{\mathcal{T}}_{\ell, \ell^{\prime}} \mid H_{\ell}^{K_{*}}, H_{\ell^{\prime}}^{K_{*}}\right] \leq \epsilon$. 
$2^{o}$ Because $H_{\ell^{\prime}}^{K *}, H_{\ell}^{K *}$ can be decided upon with risk $\leq \epsilon<1 / 2$, by detector calculus

$$
\varepsilon_{*}\left(\mathcal{P}_{\ell}^{K_{*}}, \mathcal{P}_{\ell^{\prime}}^{K_{*}}\right) \leq 2 \sqrt{\epsilon(1-\epsilon)}<1
$$

Since $\mathcal{O}$ is a simple o.s,

$$
\varepsilon_{*}\left(\mathcal{P}_{\ell}, \mathcal{P}_{\ell^{\prime}}\right) \leq[2 \sqrt{\epsilon(1-\epsilon)}]^{1 / K_{*}}<1
$$

We conclude that

$$
\theta_{K} \leq[2 \sqrt{\epsilon(1-\epsilon)}]^{K / K_{*}} L
$$

and

$$
\operatorname{Risk}^{\mathcal{C}}\left[\mathcal{T}^{K} \mid H_{1}^{\oplus, K}, \ldots, H_{L}^{\oplus, K}\right] \leq \varkappa \theta_{K} \leq \epsilon
$$

when

$$
\frac{K}{K^{*}} \geq 2 \frac{1+\ln (\varkappa L) / \ln (1 / \epsilon)}{1-\ln (4(1-\epsilon)) / \ln (1 / \epsilon)} .
$$




\section{Illustration: Selecting the Best in a Family of Estimates}

Problem: we are given

- a simple o.s. $\mathcal{O}=\left((\Omega, \pi),\left\{p_{\mu}: \mu \in \mathcal{M}\right\}, \mathcal{F}\right)$ and have access to stationary $K$-repeated observations

$$
\omega_{k} \sim p_{A\left(x_{*}\right)}(\cdot), k=1, \ldots, K,
$$

of unknown signal $x_{*}$ known to belong to a given convex compact set $X \subset \mathbb{R}^{n}$.

Here $[x \mapsto A(x)$ : affine mapping such that $A(X) \subset \mathcal{M}]$;

- $M$ candidate estimates $x_{i} \in \mathbb{R}^{n}, 1 \leq i \leq M$, of $x_{*}$,

- a norm $\|\cdot\|$ on $\mathbb{R}^{n}$, and a reliability tolerance $\epsilon \in(0,1)$ 


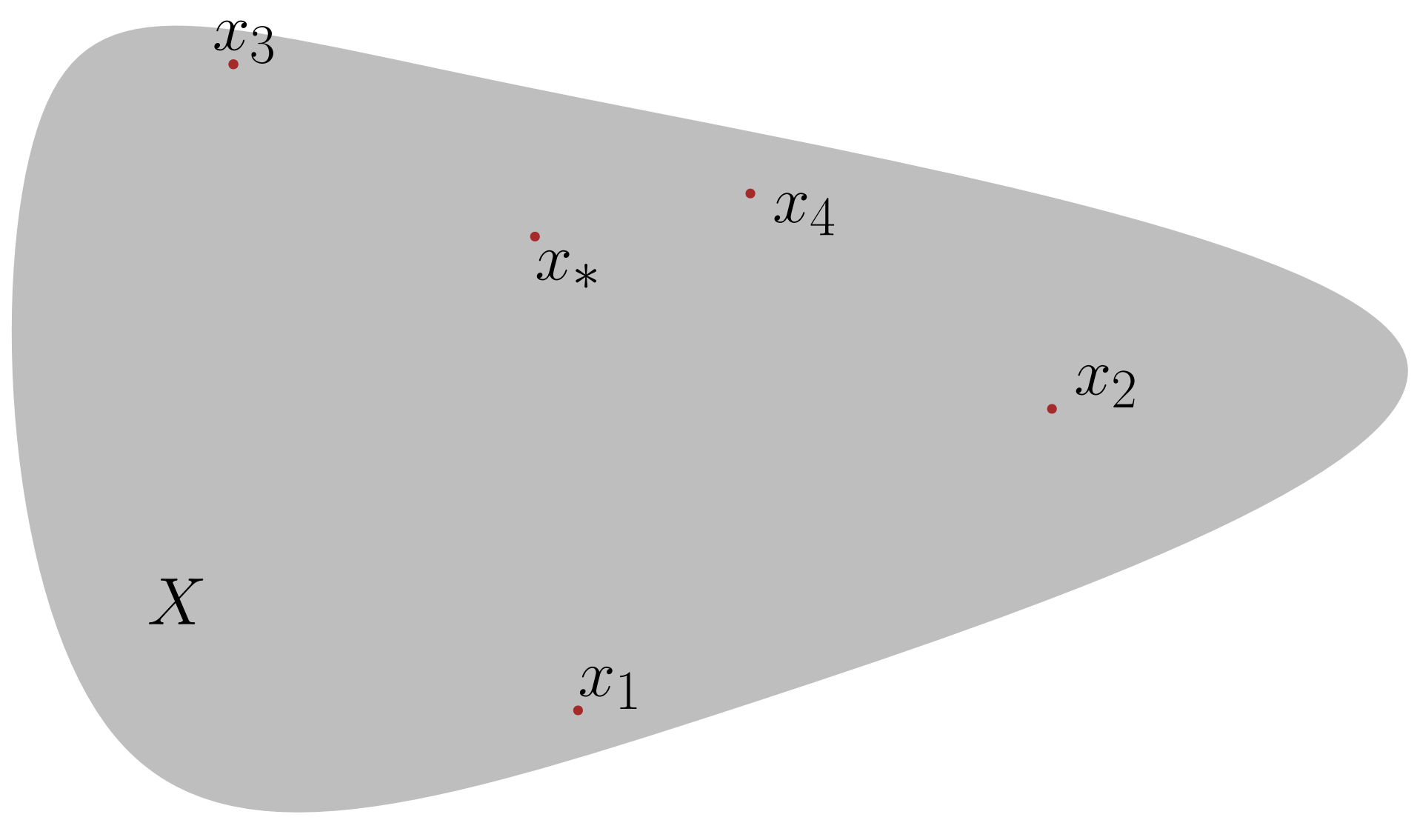

Parameter space

Given observations $\omega_{1}, \ldots, \omega_{K}$ we look to identify $(1-\epsilon)$-reliably the $\|\cdot\|$ closest to $x_{*}$ point among $x_{1}, \ldots, x_{M}$. 
Actual goal: given $\alpha \geq 1, \beta \geq 0$ and a grid

$$
\Gamma=\left\{r_{0}>r_{1}>\ldots>r_{N}>0\right\},
$$

use observations $\omega_{1}, \ldots, \omega_{K}$ to identify $(1-\epsilon)$-reliably $x_{i\left(\omega^{K}\right)}$ such that

$$
\left\|x_{*}-x_{i\left(\omega^{K}\right)}\right\| \leq \alpha \rho\left(x_{*}\right)+\beta
$$

where

$$
\rho(x):=\min \left\{r: r \in \Gamma, r \geq \min _{i}\left\|x-x_{i}\right\|\right\}
$$

is the "grid approximation" of $\min _{i}\left\|x-x_{i}\right\|$. 


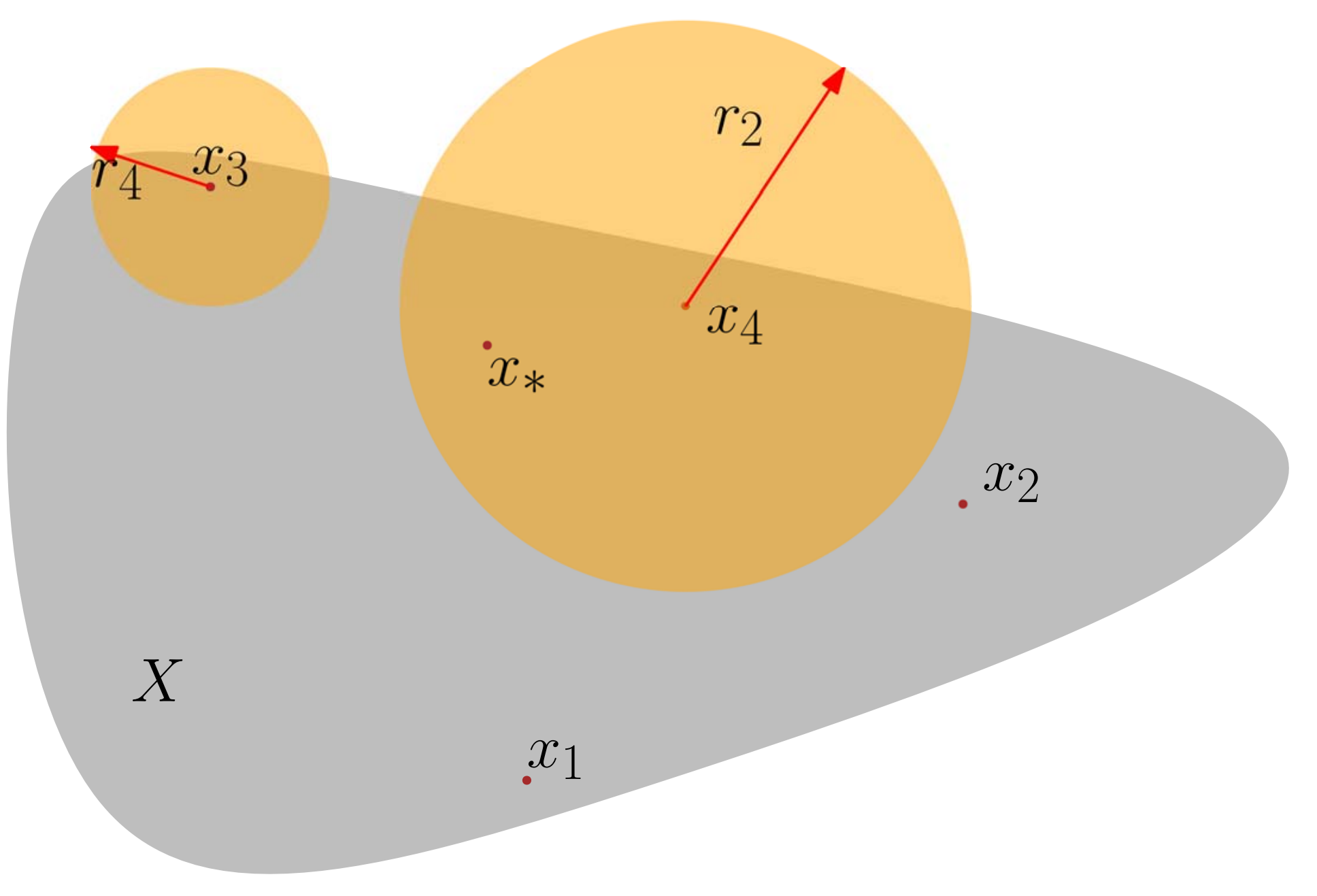

Parameter space 


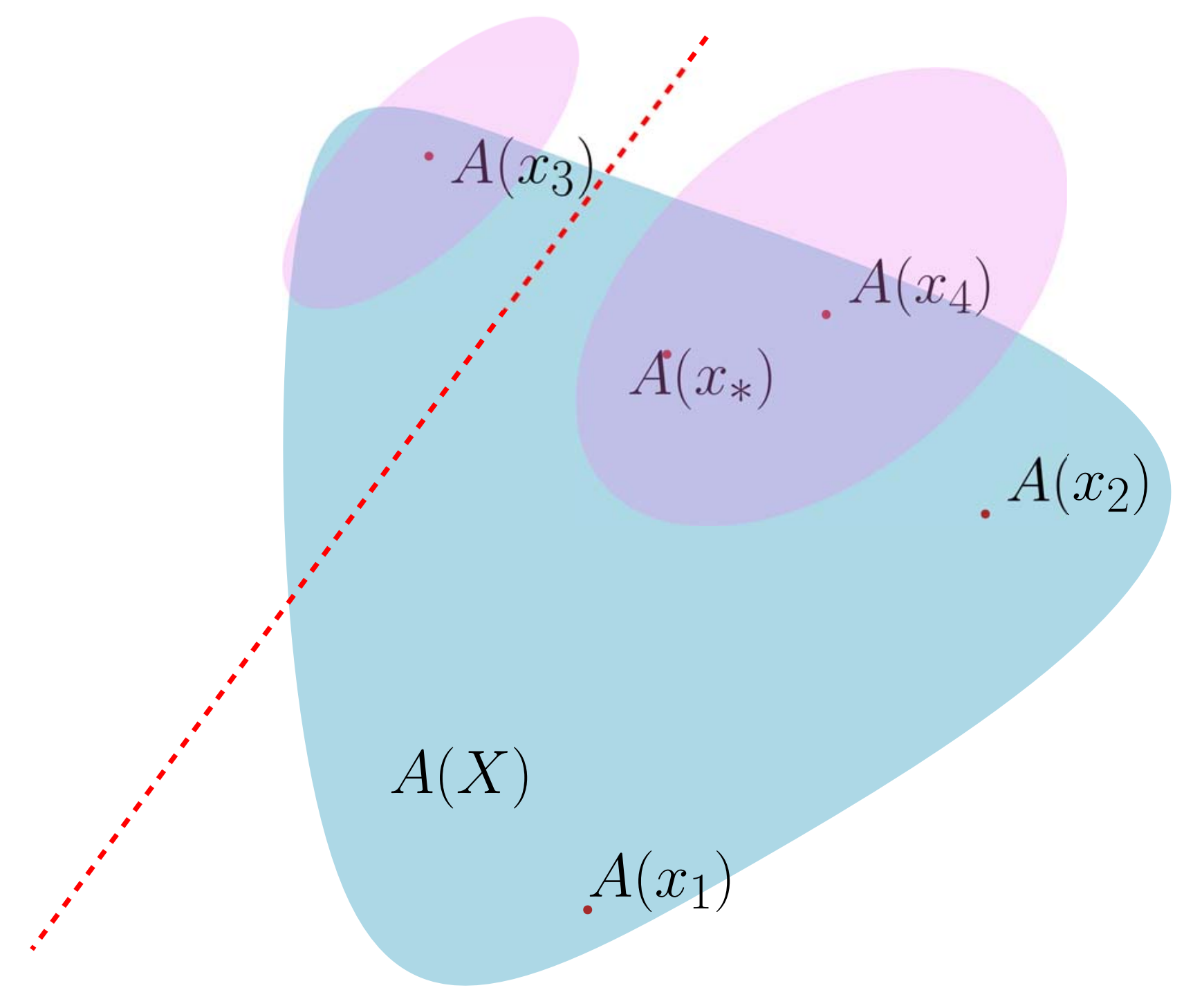

Observation parameter space 


\section{Solution:}

- Consider $M(N+1)$ hypotheses

$$
H_{i j}: \omega_{k} \sim p_{A(x)}(\cdot) \text { for some } x \in X_{i j}:=\left\{x \in X:\left\|x-x_{i}\right\| \leq r_{j}\right\} .
$$

- Define closeness $\mathcal{C}=\mathcal{C}_{\alpha, \beta}$ :

$$
[i j] \text { is } \mathcal{C} \text {-close to }\left[i^{\prime} j^{\prime}\right] \quad \text { iff }\left\|x_{i}-x_{i^{\prime}}\right\| \leq r_{j}+r_{j^{\prime}}+\beta .
$$

- Build minimum risk pairwise detectors $\phi_{i j, i^{\prime} j^{\prime}}$, along with their risks $\epsilon_{i j, i^{\prime} j^{\prime}}$, for hypotheses $H_{i j}$.

- Choose $\beta$ such that $\epsilon_{i j, i^{\prime} j^{\prime}}<1$ for all $\left(i j, i^{\prime} j^{\prime}\right) \notin \mathcal{C}$.

- Find the smallest $K=K_{\epsilon}(\beta)$ such that the $K$-observation detector-based test $\mathcal{T}^{K}$ decides on $\left\{H_{i j}: i \leq M, 0 \leq j \leq N\right\}$ with $\mathcal{C}$-risk $\leq \epsilon$.

- To build $i\left(\omega^{K}\right)$,

- apply $\mathcal{T}^{K}$ to observation $\omega^{K}$,

- select $H_{i_{*}\left(\omega^{K}\right) j_{*}\left(\omega^{K}\right)}$ with the largest $j$ among accepted hypotheses $H_{i j}$. 
Proposition. In the situation in question, assume that for some $\epsilon \in(0,1 / 2)$, $b \geq 0$ and positive integer $\bar{K}$ in the nature there exists an inference $\omega^{\bar{K}} \rightarrow$ $i_{*}\left(\omega^{\bar{K}}\right)$ such that, for all $x^{*} \in X$,

$$
\operatorname{Prob}\left\{\left\|x_{*}-x_{i_{*}\left(\omega^{\bar{K}}\right)}\right\| \leq \rho\left(x_{*}\right)+b\right\} \geq 1-\epsilon .
$$

- Then one can choose $\beta=2 b$.

- The resulting inference $\omega^{K} \mapsto i\left(\omega^{K}\right)$ satisfies

$$
\operatorname{Prob}\left\{\left\|x_{*}-x_{i\left(\omega^{K}\right)}\right\| \leq 3 \rho\left(x_{*}\right)+2 b\right\} \geq 1-\epsilon .
$$

for $K \geq K_{\epsilon}(2 b)$, where

$$
K_{\epsilon}(2 b) \leq \text { Ceil }\left(\frac{2 \ln (M(N+1)) / \ln (1 / \epsilon)}{1-\ln (4(1-\epsilon)) / \ln (1 / \epsilon)} \bar{K}\right) .
$$


Numerical illustration. Given noisy observation

$$
\omega=A x+\sigma \xi, \xi \sim \mathcal{N}\left(0, I_{n}\right)
$$

of the "discretized primitive" $A x$ of a signal $x=\left[x^{1} ; \ldots ; x^{n}\right] \in \mathbb{R}^{n}$ :

$$
[A x]_{j}=\frac{1}{n} \sum_{s=1}^{j} x^{s}, 1 \leq j \leq n,
$$

for $i=1, \ldots, \kappa$ we have built Least Squares polynomial, of order $i-1$, approximations $x_{i}$ of $x$ :

$$
\begin{gathered}
x_{i}=\operatorname{argmin}_{x \in \mathcal{P}_{i}}\|A x-\omega\|_{2}^{2} \\
{\left[\mathcal{P}_{i}=\left\{x=\left[x^{1} ; \ldots ; x^{n}\right]: x^{s} \text { is polynomial, of degree } \leq i-1, \text { in } s\right\}\right]}
\end{gathered}
$$

and now want to use $K$ additional observations to identify the nearly closest to $x_{*}$, in the norm

$$
\|u\|=\frac{1}{n} \sum_{i=1}^{n}\left|u_{i}\right|
$$

on $\mathbb{R}^{n}$, among the points $x_{i}, 1 \leq i \leq \kappa$. 
Numerical experiment $[\epsilon=0.01, n=128, \sigma=0.01, \kappa=5, \beta=0.05]$
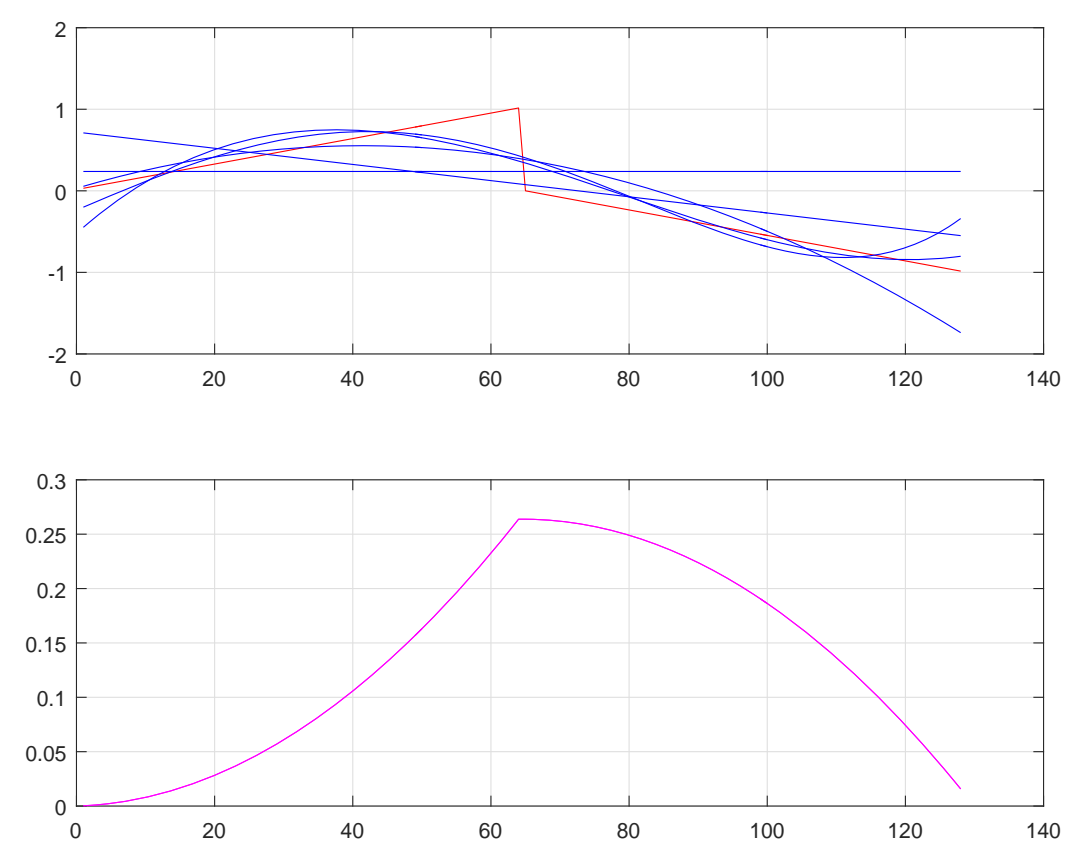

Top: $x_{*}$ and $x_{i}$. Bottom: the primitive of $x_{*}$

\begin{tabular}{||c||c|c|c|c|c||}
\hline \hline$i$ & 1 & 2 & 3 & 4 & 5 \\
\hline$\left\|x-x_{i}\right\|$ & 0.5348 & 0.33947 & 0.23342 & 0.16313 & 0.16885 \\
\hline \hline
\end{tabular}

distances from $x_{*}$ to $x_{i}$ 
- With $K=3$ (computed value), for a sample of size 1000 , the closest to $x_{*}$ point was correctly identified in all simulations

- the same was true when $K=3$ was replaced with $K=1$;

- replacing $K=3$ with $K=1$ and increasing $\sigma$ from 0.01 to 0.05 , the procedure started to make imperfect conclusions.

However, the exactly closest to $x_{*}$ point $x_{4}$ was identified correctly in as many as $96 \%$ of simulations, with estimated excess error

$$
\mathbf{E}\left\{\left\|x_{*}-x_{i\left(\omega^{1}\right)}\right\|-\rho\left(x_{*}\right)\right\} \leq 0.0024 .
$$




\section{Illustration: Recovering Linear-Fractional Function of a Signal}

Problem: An unknown signal $x$ known to belong to a given convex compact set $X \subset \mathbb{R}^{n}$ is observed according to

$$
\omega=A x+\sigma \xi, \xi \sim \mathcal{N}\left(0, I_{d}\right)
$$

Our goal is to recover the value at $x$ of a linear-fractional functional

$$
F(z)=\frac{f^{T} z}{e^{T} z}, \text { with } e^{T} z>0, z \in X .
$$

Illustration: We are given noisy measurements of voltages $V_{i}$ at some nodes $i$ and currents $I_{i j}$ in some arcs $(i, j)$ of an electric circuit, and want to recover the resistance of a particular arc $(\hat{i}, \hat{j})$ :

$$
r_{\widehat{i} \widehat{j}}=\frac{V_{\widehat{j}}-V_{\widehat{i}}}{I_{\widehat{i} \hat{j}}}
$$




\section{Circuit with 8 nodes and 11 arcs}

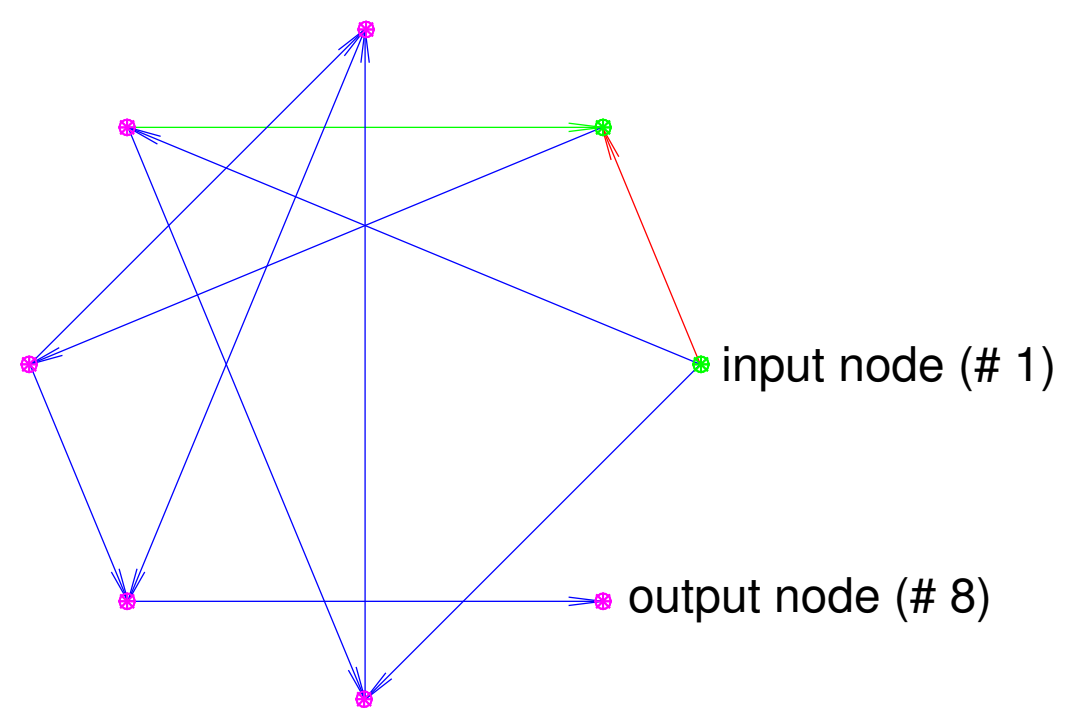

$$
\begin{gathered}
x=[\text { voltages at nodes; currents in arcs }] \\
A x=\text { observable voltages; observable currents }]
\end{gathered}
$$

- Currents are measured in blue arcs only

- Voltages are measured in magenta nodes only

- We want to recover resistance of red arc

- $X:\left\{\begin{array}{l}\text { conservation of current, except for nodes \#\#1,8 } \\ \text { zero voltage at node \#1, nonnegative currents } \\ \text { current in red arc at least 1, total of currents at most } 33 \\ \text { Ohm Law, resistances of arcs between } 1 \text { and } 10\end{array}\right.$ 


\section{Strategy: Given $L$,}

- split the range

$$
\Delta=\left[\min _{x \in X} F(x), \max _{x \in X} F(x)\right]
$$

into $L$ consecutive bins $\Delta_{\ell}$ of length $\delta_{L}=$ length $(\Delta) / L$,

- define convex compact sets

$$
X_{\ell}=\left\{x \in X: F(x) \in \Delta_{\ell}\right\}, \quad M_{\ell}=\left\{A x: x \in X_{\ell}\right\}, 1 \leq \ell \leq L
$$

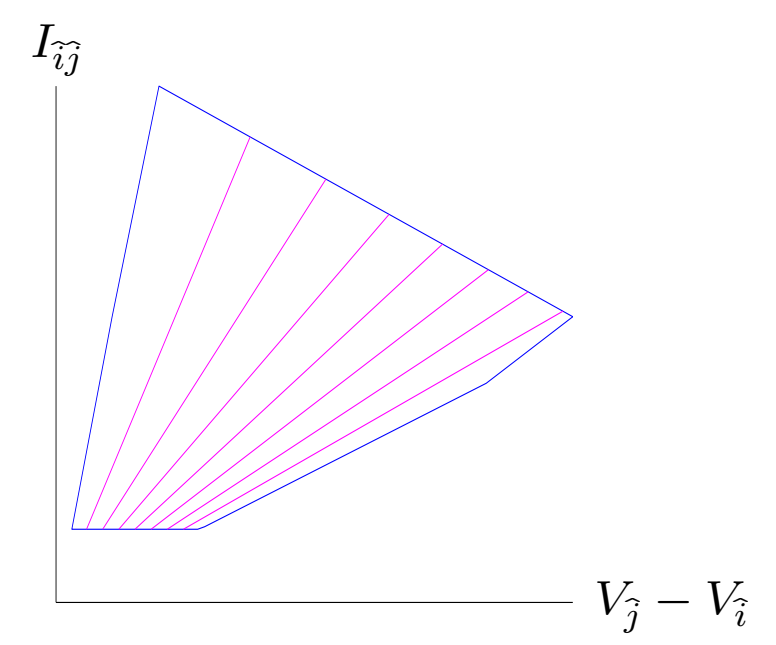

a 2D projection of $X$ and $X_{1}, \ldots, X_{8}$ 
- We are to decide on $L$ hypotheses

$$
H_{\ell}: P=\mathcal{N}\left(\mu, \sigma^{2} I\right), \quad \mu \in M_{\ell}
$$

about the distribution $P$ of observation

$$
\omega=A x+\sigma \xi
$$

up to closeness

$$
\mathcal{C}: \text { : } H_{\ell} \text { is close to } H_{\ell^{\prime}} \text { if and only if }\left|\ell-\ell^{\prime}\right| \leq 1 \text { " }
$$

- Estimate $F(x)$ by the barycenter of accepted bins.

Fact: For the resulting test $\mathcal{T}$, with probability

$$
\geq 1-\operatorname{Risk}^{\mathcal{C}}\left[\mathcal{T} \mid H_{1}, \ldots, H_{L}\right]
$$

the estimation error does not exceed $\delta_{L}$. 
Implementation and results: Given target risk $\epsilon$ and $L$, we select the largest $\sigma$ for which $\operatorname{Risk}^{\mathcal{C}}\left[\mathcal{T} \mid H_{1}, \ldots, H_{L}\right]$ is $\leq \epsilon$.

- This is what we get for $\epsilon=0.01: \Delta=[1,10]$

\begin{tabular}{||c||c|c|c||}
\hline \hline$L$ & 8 & 16 & 32 \\
\hline \hline$\delta_{L}$ & $9 / 8 \approx 1.13$ & $9 / 16 \approx 0.56$ & $9 / 32 \approx 0.28$ \\
\hline$\sigma$ & 0.024 & 0.010 & 0.005 \\
\hline$\sigma_{\text {opt }} / \sigma \leq$ & 1.31 & 1.31 & 1.33 \\
\hline \hline$\sigma \sigma$ & 0.031 & 0.013 & 0.006 \\
\hline$\sigma_{\text {opt }} / \sigma \leq$ & 1.01 & 1.06 & 1.08 \\
\hline \hline
\end{tabular}

- $\sigma_{\text {opt }}$ - the largest $\sigma$ for which "in the nature" there exists a test deciding on $H_{1}, \ldots, H_{L}$ with $\mathcal{C}$-risk $\leq 0.01$

- Red data: Risks $\epsilon_{\ell \ell^{\prime}}$ of pairwise tests are bounded via risks of optimal detectors, $\mathcal{C}$-risk of $\mathcal{T}$ is bounded by

$$
\left\|\left[\epsilon_{\ell \ell^{\prime}} \cdot \chi_{\left(\ell, \ell^{\prime}\right) \notin \mathcal{C}}\right]_{\ell, \ell^{\prime}=1}^{L}\right\|_{2,2} ;
$$

- Black data: Risks $\epsilon_{\ell \ell^{\prime}}$ of pairwise tests are bounded via error function, $\mathcal{C}$-risk of $\mathcal{T}$ is bounded by

$$
\max _{\ell} \sum_{\ell^{\prime}:\left(\ell, \ell^{\prime}\right) \notin \mathcal{C}} \epsilon_{\ell \ell^{\prime}}
$$




\section{Recovering $\mathrm{N}$-Convex Functionals on Unions of Convex Sets}

Fact: The construction used to recover linear-fractional function can be extended to recovering $\mathrm{N}$-convex functionals.

Definition: Let $X \subset \mathbb{R}^{n}$ be a convex compact set, $F: X \rightarrow \mathbb{R}$ be a continuous function, and $N$ be a positive integer. We say that $F$ is $N$-convex, if for every real $a$ the sets

$$
X^{a, \geq}=\{x \in X: F(x) \geq a\}, X^{a, \leq}=\{x \in X: F(x) \leq a\}
$$

can be represented as the unions of at most $N$ convex compact sets.

Example A: Fractional-linear function $F(x)=\frac{e(x)}{d(x)}$ with positive on $X$ denominator is 1-convex:

$$
\left\{x \in X: F(x){ }_{\leq}^{\geq} a\right\}=\left\{x \in X: e(x)-a d(x){ }_{\leq}^{\geq} 0\right\}
$$


Example B: If $F_{i}$ is $N_{i}$-convex on $X, i=1,2$, then $\max \left[F_{1}, F_{2}\right]$ and $\min \left[F_{1}, F_{2}\right]$ are $\max \left[N_{1}+N_{2}, N_{1} N_{2}\right]$-convex on $X$ :

$$
\begin{aligned}
& \left\{\begin{array}{l}
X_{i}^{a, \geq}:=\left\{x \in X: F_{i}(x) \geq a\right\}=\bigcup_{\nu=1}^{N_{i}} U_{\nu, i}^{a} \\
X_{i}^{a, \leq}:=\left\{x \in X: F_{i}(x) \leq a\right\}=\bigcup_{\nu=1}^{N_{i}} V_{\nu, i}^{a}
\end{array}, i=1,2[U, V: \text { convex] }\right. \\
& \Rightarrow\left\{\begin{array}{l}
\left\{x \in X: \max \left[F_{1}(x), F_{2}(x)\right] \geq a\right\}=\left[\bigcup_{\mu \leq N_{1}} U_{\mu, 1}^{a}\right] \cup\left[\underset{\nu \leq N_{2}}{\cup} U_{\nu, 2}^{a}\right] \\
\left\{x \in X: \max \left[F_{1}(x), F_{2}(x)\right] \leq a\right\}=\bigcup_{\mu \leq N_{1}, \nu \leq N_{2}}\left[V_{\mu, 1}^{a} \cap V_{\nu, 2}^{a}\right]
\end{array}\right.
\end{aligned}
$$

Extension: If $F_{i}$ is $N_{i}$-convex on $X, i=1,2, \ldots, M$, then $\max \left[F_{1}, F_{2}, \ldots, F_{M}\right]$ and $\min \left[F_{1}, F_{2}, \ldots, F_{M}\right]$ are $\max \left[\sum_{i} N_{i}, \prod_{i} N_{i}\right]$-convex on $X$. 


\section{Problem of interest: Given}

- convex compact set $X \subset \mathbb{R}^{n}$,

- $N$-convex functional $F: X \rightarrow \mathbb{R}$,

- a collection $X_{j}, j=1, \ldots, J$, of convex compact subsets of $X$,

- stationary $K$-repeated observations $\omega_{1}, \ldots, \omega_{K}$ stemming, via simple o.s., from unknown signal $x \in \bigcup_{j=1}^{J} X_{j}$,

we want to recover $F(x)$. 
Strategy: Given $L$, we

- split the range

$$
\Delta=\left[\min _{x \in X} F(x), \max _{x \in X} F(x)\right]
$$

into $L$ consecutive bins $\Delta_{\ell}$ of length $\delta_{L}=\operatorname{length}(\Delta) / L$,

- and observe that by $N$-convexity of $F$, each set

$$
\left\{x \in \bigcup_{j=1}^{J} X_{j}: F(x) \in \Delta_{\ell}\right\}
$$

is the union of at most $N^{2} J$ convex compact sets $Y_{s}^{\ell}$;

- Associate with nonempty sets $Y_{s}^{\ell}$ the hypotheses "observation stems from a signal from $Y_{s}^{\ell,}$

- Define closeness $\mathcal{C}$ on the resulting collection of hypotheses $H_{1}, \ldots, H_{\mathcal{L}}$, $\mathcal{L} \leq N^{2} J L$ :

$$
H_{\mu} \text { and } H_{\nu} \text { are } \mathcal{C} \text {-close }
$$

iff both hypotheses stem from the same or from two consecutive bins $\Delta_{\ell}$ 
- Use our gear for testing multiple convex hypotheses in simple o.s. to build a test $\mathcal{T}_{K}$ deciding on $H_{1}, \ldots, H_{\mathcal{L}}$ up to closeness $\mathcal{C}$ via $K$-repeated observation.

- We apply the test $\mathcal{T}_{K}$ to observations $\omega_{1}, \ldots, \omega_{K}$ and take as the estimate of $F(x)$ the center of masses of all bins associated with the hypotheses accepted by the test.

Same as in the above fractional-linear example, it is immediately seen that The probability for the recovery error to be $>\delta_{L}$ is at most the $\mathcal{C}$-risk of $\mathcal{T}_{K}$.

In addition, with our estimate, the number of observations $K$ required to ensure recovery error $\leq \delta_{L}$ with a given reliability $1-\epsilon, \epsilon \ll 1$, is within logarithmic in $N, J, L$ factor off the "ideal" number of observations needed to achieve, with reliability $1-\epsilon$, recovery error $\delta_{L} / 2$. 


\section{Recovering Linear Functionals on Unions of Convex Sets Revisited}

\section{Problem of interest: Given}

- simple o.s. $\mathcal{O}=\left((\Omega, \Pi),\left\{p_{\mu}(\cdot): \mu \in \mathcal{M}\right\}, \mathcal{F}\right)$

- collection $X_{j}, j=1, \ldots, J$, of nonempty convex compact sets in $\mathbb{R}^{n}$

- affine sensing mappings $A_{j}(\cdot)$ such that

$$
A_{j}(x) \in \mathcal{M} \forall x \in X_{j}
$$

- linear function $g^{T} x$ on $\mathbb{R}^{n}$,

- stationary $K$-repeated observation $\omega^{K}=\left(\omega_{1}, \ldots, \omega_{K}\right), \omega_{k} \sim p_{A_{j x}(x)}$, stemming from unknown signal $x \in X=\cup_{j} X_{j}$, such that $x \in X_{j_{x}}$ we want to estimate $g^{T} x$.

Note: When all sensing mappings $A_{j}(\cdot)$ are identical to each other, our problem becomes a special case of recovering 1-convex function on the union of convex sets. If, furthermore, $J=1$ (there just one set $X=X_{1}$ ), we come back to the classical problem of linear functional estimation. 
Risk: We quantify the performance of an estimate $\widehat{g}\left(\omega^{K}\right)$ by its $\epsilon$-risk:

$\operatorname{Risk}_{\epsilon}[\widehat{g} \mid X]=\min \left\{\rho: \operatorname{Prob}_{\omega^{K}} \sim p_{A_{j}(x)}^{K}\left\{\left|\widehat{g}\left(\omega^{K}\right)-g^{T} x\right|>\rho\right\} \leq \epsilon \forall\left(j \leq J, x \in X_{j}\right)\right\}$

- the smallest guaranteed size of $(1-\epsilon)$-confidence interval of the estimate.

- $\epsilon \in(0,1)$ : once for ever fixed tolerance.

We consider estimates which are aggregates of "pairwise" estimates of the form

$$
\phi_{i j}^{(K)}\left(\omega^{K}\right)=\sum_{k} \phi_{i j}\left(\omega_{k}\right)
$$

with $\phi_{i j} \in \mathcal{F}$. 
Question: Given $\phi \in \mathcal{F}, \delta \in(0,1), \rho>0$, and $i, j$, how to certify that

$$
\begin{aligned}
& \operatorname{Prob}_{\omega^{K} \sim p_{A_{i}(x)}^{K}}\left\{\sum_{k} \phi\left(\omega_{k}\right)>g^{T} x+\rho\right\} \leq \delta \forall x \in X_{i} \\
& \operatorname{Prob}_{\omega^{K} \sim p_{A_{j}(v)}^{K}}\left\{\sum_{k} \phi\left(\omega_{k}\right)<g^{T} y-\rho\right\} \leq \delta \forall y \in X_{j}
\end{aligned}
$$

Answer: Define the "aggregate"

$$
\Phi_{\mathcal{O}}(\phi, \mu)=\ln \left(\int \exp \{\phi(\omega)\} p_{\mu}(\omega) \Pi(d \omega)\right): \mathcal{F} \times \mathcal{M} \rightarrow \mathbb{R}
$$

By construction, $\Phi_{\mathcal{O}}(\cdot, \cdot)$ is convex in $\phi$ and concave in $\mu$.

A sufficient condition for $(a)$ and $(b)$ is existence of $\alpha>0$ such that

$$
\begin{array}{r}
\max _{x \in X_{i}}\left[K \alpha \Phi_{\mathcal{O}}\left(\phi / \alpha, A_{i}(x)\right)-g^{T} x+\alpha \ln (1 / \delta)\right] \leq \rho \\
\max _{y \in X_{j}}\left[K \alpha \Phi_{\mathcal{O}}\left(-\phi / \alpha, A_{i}(x)\right)+g^{T} y+\alpha \ln (1 / \delta)\right] \leq \rho
\end{array}
$$


Indeed when (c) takes place and $x \in X_{i}$, we have

$$
\begin{aligned}
& \pi:=\operatorname{Prob}_{\omega^{K} \sim p_{A_{i}(x)}^{K}}\left\{\sum_{k} \phi\left(\omega_{k}\right)>g^{T} x+\rho\right\} \\
& \leq \mathbf{E}_{\omega^{K} \sim p_{A_{i}(x)}^{K}}\left\{\exp \left(\frac{1}{\alpha}\left[\sum_{k} \phi\left(\omega_{k}\right)-g^{T} x-\rho\right]\right)\right\} \\
&= \exp \left(-\left[g^{T} x+\rho\right] / \alpha\right)\left[\mathbf{E}_{\omega \sim p_{A_{i}(x)}}\{\exp (\phi(\omega) / \alpha)\}\right]^{K} \\
&= \exp \left(\left[g^{T} x+\rho\right] / \alpha\right) \exp \left\{K \Phi_{\mathcal{O}}\left(\phi / \alpha, A_{i}(x)\right)\right\} \\
& \Rightarrow \quad \ln (\pi) \leq K \Phi_{\mathcal{O}}\left(\phi / \alpha, A_{i}(x)\right)-\alpha^{-1}\left[g^{T} x+\rho\right] \\
& \Rightarrow \quad \alpha \ln (\pi) \leq K \alpha \Phi_{\mathcal{O}}\left(\phi / \alpha, A_{i}(x)\right)-g^{T} x-\rho \\
& \quad \Rightarrow[\operatorname{by}(c)] \alpha \ln (\pi) \leq-\alpha \ln (1 / \delta) \Rightarrow \pi \leq \delta
\end{aligned}
$$

Thus, (c) implies (a); in the same way, $(d)$ implies $(b)$. 


$$
\begin{gathered}
\mathcal{O}=\left((\Omega, \Pi),\left\{p_{\mu}(\cdot): \mu \in \mathcal{M}\right\}, \mathcal{F}\right) \\
\Phi_{\mathcal{O}}(\phi, \mu)=\ln \left(\int \exp \{\phi(\omega)\} p_{\mu}(\omega) \Pi(d \omega)\right): \mathcal{F} \times \mathcal{M} \rightarrow \mathbb{R} \\
\left\{\begin{array}{c}
\max _{x \in X_{i}}\left[K \alpha \Phi_{\mathcal{O}}\left(\phi / \alpha, A_{i}(x)\right)-g^{T} x+\alpha \ln (1 / \delta)\right] \leq \rho \\
\max _{y \in X_{j}}\left[K \alpha \Phi_{\mathcal{O}}\left(-\phi / \alpha, A_{i}(x)\right)+g^{T} y+\alpha \ln (1 / \delta)\right] \leq \rho
\end{array}\right. \\
\Rightarrow\left\{\begin{array}{c}
\operatorname{Prob}_{\omega^{K} \sim p_{A_{i}(x)}^{K}}\left\{\sum_{k} \phi\left(\omega_{k}\right)>g^{T} x+\rho\right\} \leq \epsilon / 2 \forall x \in X_{i} \\
\operatorname{Prob}_{\omega^{K} \sim p_{A_{j}(y)}^{K}}\{(a)
\end{array}\right.
\end{gathered}
$$

Observation: Let $\rho>0, \psi \in \mathcal{F}$ and $\alpha>0$ be such that

$$
\max _{x \in X_{i}, y \in X_{j}} \frac{1}{2}\left[K \alpha\left[\Phi_{\mathcal{O}}\left(\psi / \alpha, A_{i}(x)\right)+\Phi_{\mathcal{O}}\left(-\psi / \alpha, A_{j}(y)\right)\right]+g^{T}[y-x]\right]+\alpha \ln (1 / \delta) \leq \rho
$$

\section{Then, setting}

$$
\begin{gathered}
\varkappa=\frac{1}{2 K}[\underbrace{\max _{y \in X_{j}}\left[K \alpha \Phi_{\mathcal{O}}\left(-\psi / \alpha, A_{j}(y)\right)+g^{T} y\right]}_{\gamma_{-}}-\underbrace{\max _{x \in X_{i}}\left[K \alpha \Phi_{\mathcal{O}}\left(\psi / \alpha, A_{i}(x)\right)-g^{T} x\right]}_{\gamma_{+}}], \\
\phi(\cdot)+\varkappa
\end{gathered}
$$

so that $\phi \in \mathcal{F}$, we ensure $(c)$ and $(d)$ and thus ensure $(a),(b)$. 
Indeed, $(*)$ reads $\frac{1}{2}\left[\gamma_{+}+\gamma_{-}\right]+\alpha \ln (1 / \delta) \leq \rho$, and clearly

$$
\Phi_{\mathcal{O}}(\phi / \alpha, \cdot)=\Phi_{\mathcal{O}}(\psi / \alpha, \cdot)+\varkappa / \alpha, \Phi_{\mathcal{O}}(-\phi / \alpha, \cdot)=\Phi_{\mathcal{O}}(-\psi / \alpha, \cdot)-\varkappa / \alpha,
$$

whence

$$
\begin{aligned}
& \max _{x \in X_{i}}\left[K \alpha \Phi_{\mathcal{O}}\left(\phi / \alpha, A_{i}(x)\right)-g^{T} x+\alpha \ln (1 / \delta)\right] \\
& \quad=\gamma_{+}+K \varkappa+\alpha \ln (1 / \delta)=\gamma_{+}+\frac{1}{2}\left[\gamma_{-}-\gamma_{+}\right]+\alpha \ln (1 / \delta) \\
& \quad=\frac{1}{2}\left[\gamma_{+}+\gamma_{-}\right]+\alpha \ln (1 / \delta) \leq \rho,
\end{aligned}
$$

and

$$
\begin{aligned}
& \max _{y \in X_{j}}\left[K \alpha \Phi_{\mathcal{O}}\left(-\phi / \alpha, A_{i}(x)\right)+g^{T} y+\alpha \ln (1 / \delta)\right] \\
& \quad=\gamma_{-}-K \varkappa+\alpha \ln (1 / \delta)=\gamma_{-}-\frac{1}{2}\left[\gamma_{-}-\gamma_{+}\right]+\alpha \ln (1 / \delta) \\
& \quad=\frac{1}{2}\left[\gamma_{+}+\gamma_{-}\right]+\alpha \ln (1 / \delta) \leq \rho
\end{aligned}
$$


Building the estimate: Consider convex optimization problems

$$
\begin{aligned}
\operatorname{Opt}_{i j}(K)=\min _{\alpha>0, \psi \in \mathcal{F}}\left\{\operatorname { m a x } _ { x \in X _ { i } , y \in X _ { j } } \frac { 1 } { 2 } \left[K \alpha\left[\Phi_{\mathcal{O}}\left(\psi / \alpha, A_{i}(x)\right)+\Phi_{\mathcal{O}}\left(-\psi / \alpha, A_{i}(y)\right)\right]\right.\right. \\
\left.\left.+g^{T}[y-x]\right]+\alpha \ln (2 J / \epsilon)\right\} .
\end{aligned}
$$

Let $\alpha_{i j}, \psi_{i j}$ be feasible solutions to the problems, and $\rho_{i j}$ be the corresponding values of the objectives (the less, the better).

- We set

$$
\begin{aligned}
\varkappa_{i j}= & \frac{1}{2 K}\left[\max _{y \in X_{j}}\left[K \alpha_{i j} \Phi_{\mathcal{O}}\left(-\psi_{i j} / \alpha_{i j}, A_{j}(y)\right)+g^{T} y\right]\right. \\
& \left.-\max _{x \in X_{i}}\left[K \alpha_{i j} \Phi_{\mathcal{O}}\left(\psi_{i j} / \alpha_{i j}, A_{i}(x)\right)-g^{T} x\right]\right], \\
\phi_{i j}(\cdot)= & \psi_{i j}(\cdot)+\varkappa_{i j}, \quad \phi_{i j}^{(K)}\left(\omega^{K}\right)=\sum_{k} \phi_{i j}\left(\omega_{k}\right),
\end{aligned}
$$

thus ensuring

$$
\begin{aligned}
& \operatorname{Prob}_{\omega^{K} \sim p_{A_{i}(x)}^{K}}\left\{\phi_{i j}^{(K)}\left(\omega^{K}\right)>g^{T} x+\rho_{i j}\right\} \leq \frac{\epsilon}{2 J}, \quad x \in X_{i} \\
& \operatorname{Prob}_{\omega^{K} \sim p_{A_{j}(y)}^{K}}\left\{\phi_{i j}^{(K)}\left(\omega^{K}\right)<g^{T} y-\rho_{i j}\right\} \leq \frac{\epsilon}{2 J}, y \in X_{j}
\end{aligned}
$$




$$
\begin{aligned}
& \operatorname{Prob}_{\omega^{K} \sim p_{A_{i}(x)}^{K}}\left\{\phi_{i j}^{(K)}\left(\omega^{K}\right)>g^{T} x+\rho_{i j}\right\} \leq \frac{\epsilon}{2 J}, x \in X_{i} \\
& \operatorname{Prob}_{\omega^{K} \sim p_{A_{j}(y)}^{K}(y)}\left\{\phi_{i j}^{(K)}\left(\omega^{K}\right)<g^{T} y-\rho_{i j}\right\} \leq \frac{\epsilon}{2 J}, y \in X_{j}
\end{aligned}
$$

- Given observation $\omega^{K}$, we build the matrix

$$
\left[E_{i j}:=\phi_{i j}^{(K)}\left(\omega^{K}\right)\right]_{i, j \leq J}
$$

set

$$
\begin{aligned}
& r_{i}=\max _{j} E_{i j}, \quad r=\min _{i} r_{i}=\min _{i} \max _{j} E_{i j} \\
& c_{j}=\min _{i} E_{i j}, \quad c=\max _{j} c_{j}=\max _{j} \min _{i} E_{i j}
\end{aligned}
$$

and specify the estimate $\widehat{g}\left(\omega^{K}\right)$ of $g^{T} x, x \in X=\cup_{j} X_{j}$, as

$$
\widehat{g}\left(\omega^{K}\right)=\frac{1}{2}[r+c] \text {. }
$$

Theorem $\epsilon$-Risk of the estimate $\hat{g}$ satisfies

$$
\operatorname{Risk}_{\epsilon}[\widehat{g} \mid X] \leq \rho:=\max _{i, j} \rho_{i j}
$$




$$
\begin{gathered}
\operatorname{Prob}_{\omega^{K} \sim p_{A_{i}(x)}^{K}}\left\{\phi_{i j}^{(K)}\left(\omega^{K}\right)>g^{T} x+\rho_{i j}\right\} \leq \frac{\epsilon}{2 J}, x \in X_{i} \\
\operatorname{Prob}_{\omega^{K} \sim p_{A_{j}(y)}^{K}}\left\{\phi_{i j}^{(K)}\left(\omega^{K}\right)<g^{T} y-\rho_{i j}\right\} \leq \frac{\epsilon}{2 J}, y \in X_{j} \\
E_{i j}=\phi_{i j}^{(K)}\left(\omega^{K}\right) \\
r_{i}=\max _{j} E_{i j}, \quad r=\min _{i} r_{i}=\min _{i} \max _{j} E_{i j}, \quad c_{j}=\min _{i} E_{i j}, \quad c=\max _{j} c_{j}=\max _{j} \min _{i} E_{i j} \\
\widehat{g}\left(\omega^{K}\right)=\frac{1}{2}[r+c]
\end{gathered}
$$

Proof. $1^{0}$. By $(b)$,

$$
r_{i} \geq E_{i j} \geq c_{j} \Rightarrow r_{i} \geq c_{j} \forall i, j
$$

$2^{0}$. Let observation $\omega^{K}$ stem from signal $x \in X_{\ell}$, let $P$ be the distribution of $\omega^{K}$, and let $\mathcal{E}$ be the event

$$
\phi_{\ell j}^{(K)}\left(\omega^{K}\right) \leq g^{T} x+\rho_{\ell j} \forall j \leq J \text { and } \phi_{i \ell}^{(K)}\left(\omega^{K}\right) \geq g^{T} x-\rho \forall i \leq J,
$$

so that by $(a)$ and union bound,

$$
P(\mathcal{E}) \geq 1-\epsilon .
$$

Let $\Delta=\left[g^{T} x-\rho, g^{T} x+\rho\right]$. All we need to verify is that when $\omega^{K} \in \mathcal{E}$, one has $r, c \in \Delta$. Indeed, when $\omega^{K} \in \mathcal{E}$, we have

$$
r_{\ell j} \leq g^{T} x+\rho_{\ell j} \forall j \Rightarrow r_{\ell} \leq g^{T} x+\rho \Rightarrow r \leq g^{T} x+\rho,
$$

same as

$$
c_{i \ell} \geq g^{T} x-\rho_{i \ell} \forall i \Rightarrow c_{\ell} \geq g^{T} x-\rho \Rightarrow c \geq g^{T} x-\rho .
$$

Since $r \geq c$ by $(e)$, we conclude that $r, c \in \Delta$ whenever $\omega^{K} \in \mathcal{E}$. 


\section{Near-Optimality}

$$
\begin{gathered}
\operatorname{Opt}_{i j}(K)=\min _{\alpha>0, \psi \in \mathcal{F}}\left\{\operatorname { m a x } _ { x \in X _ { i } , y \in X _ { j } } \frac { 1 } { 2 } \left[K \alpha\left[\Phi_{\mathcal{O}}\left(\psi / \alpha, A_{i}(x)\right)+\Phi_{\mathcal{O}}\left(-\psi / \alpha, A_{i}(y)\right)\right]\right.\right. \\
\left.\left.+g^{T}[y-x]\right]+\alpha \ln (2 J / \epsilon)\right\} .
\end{gathered}
$$

Note: Every $\operatorname{Opt}_{i j}(K)$ is either a real, or $-\infty$. It is immediately seen that $\operatorname{Opt}_{i i}(K)$ is a nonnegative real, so that the quantity

$$
\operatorname{Opt}(K)=\max _{i, j} \operatorname{Opt}_{i j}(K)
$$

is a nonnegative real. Properly selecting feasible solutions to the convex optimization problems $\left(P_{i j}\right)$, we can make the upper bound $\rho$ on $\epsilon$-risk of our estimate arbitrarily close to $\operatorname{Opt}(K)$.

We are about to demonstrate that $\operatorname{Opt}(K)$ is nearly the optimal $\epsilon$-risk in our estimation problem

$$
\operatorname{RiskOpt}_{\epsilon}(K)=\inf _{\widehat{g}} \operatorname{Risk}_{\epsilon}[\widehat{g} \mid X]
$$

the infimum being taken over all $K$-observation estimates. 
Theorem Let $\epsilon \in(0,1 / 2)$ and $M$ be a positive integer. Then

(i) for every integer $K$ satisfying

$$
\frac{K}{M}>\frac{2 \ln (2 J / \epsilon)}{\ln \left([4 \epsilon(1-\epsilon)]^{-1}\right)}
$$

$$
[\rightarrow 2 \text {, as } \epsilon \rightarrow+0]
$$

one has

$$
\operatorname{Opt}(K) \leq \operatorname{RiskOpt}_{\epsilon}(M) .
$$

(ii) In addition, in the special case where for every $i, j$ there exists $\bar{x}_{i j} \in$ $X_{i} \cap X_{j}$ such that $A_{i}\left(\bar{x}_{i j}\right)=A_{j}\left(\bar{x}_{i j}\right)$ one has

$$
K \geq M \Rightarrow \operatorname{Opt}(K) \leq \frac{2 \ln (2 J / \epsilon)}{\ln \left([4 \epsilon(1-\epsilon)]^{-1}\right)} \operatorname{RiskOpt}_{\epsilon}(M) .
$$


$\operatorname{Opt}_{i j}(K)=\inf _{\alpha>0, \psi \in \mathcal{F}}\left\{\max _{x \in X_{i}, y \in X_{j}} \frac{1}{2}\left[K \alpha\left[\Phi_{\mathcal{O}}\left(\psi / \alpha, A_{i}(x)\right)+\Phi_{\mathcal{O}}\left(-\psi / \alpha, A_{i}(y)\right)\right]+g^{T}[y-x]\right]+\alpha \ln (2 J / \epsilon)\right\}$

Proof of $(i)$ : Let us fix $i$ and $j . \operatorname{Opt}_{i j}(K)$ is the saddle point value in the saddle point problem

$\inf _{\alpha>0, \psi \in \mathcal{F}(x, y) \in X_{i} \times X_{j}} \max _{2}\left[\frac{1}{2} K \alpha\left[\Phi_{\mathcal{O}}\left(\psi / \alpha, A_{i}(x)\right)+\Phi_{\mathcal{O}}\left(-\psi / \alpha, A_{i}(y)\right)\right]+\frac{1}{2} g^{T}[y-x]+\alpha \ln (2 J / \epsilon)\right]$

which is convex-concave with compact maximization domain

$\Rightarrow$ [by Sion-Kakutani Theorem]

$$
\begin{gathered}
\operatorname{Opt}_{i j}(K)=\max _{(x, y) \in X_{i} \times X_{j}} Q_{i j K}(x, y), \\
Q_{i j K}(x, y)=\inf _{\alpha>0, \psi \in \mathcal{F}}\left[\frac{1}{2} K \alpha\left[\Phi_{\mathcal{O}}\left(\psi / \alpha, A_{i}(x)\right)+\Phi_{\mathcal{O}}\left(-\psi / \alpha, A_{i}(y)\right)\right]+\alpha \ln (2 J / \epsilon)\right]+\frac{1}{2} g^{T}[y-x]
\end{gathered}
$$

Setting $\phi=\psi / \alpha, \mu=A_{i}(x), \nu=A_{j}(y)$, and recalling what $\Phi_{\mathcal{O}}$ is, we get

$$
\begin{aligned}
Q_{i j K}(x, y)= & \inf _{\alpha>0} \alpha\left[\ln (2 J / \epsilon)+\inf _{\phi \in \mathcal{F}} \frac{K}{2}\left[\ln \left(\int \mathrm{e}^{\phi(\omega)} p_{\mu}(\omega) \Pi(d \omega)\right)+\ln \left(\int \mathrm{e}^{-\phi(\omega)} p_{\nu}(\omega) \Pi(d \omega)\right)\right]\right] \\
& +\frac{1}{2} g^{T}[y-x] .
\end{aligned}
$$


$Q_{i j K}(x, y)=\inf _{\alpha>0} \alpha\left[\ln (2 J / \epsilon)+\inf _{\phi \in \mathcal{F}} \frac{K}{2}\left[\ln \left(\int \mathrm{e}^{\phi(\omega)} p_{\mu}(\omega) \Pi(d \omega)\right)+\ln \left(\int \mathrm{e}^{-\phi(\omega)} p_{\nu}(\omega) \Pi(d \omega)\right)\right]\right]+\frac{1}{2} g^{T}[y-x]$

We claim that the inner inf $_{\phi}$ is attained at

$$
\phi(\omega)=\phi_{*}(\omega):=\frac{1}{2} \ln \left(p_{\nu}(\omega) / p_{\mu}(\omega)\right)
$$

(note that $\phi_{*} \in \mathcal{F}$ since $\mathcal{O}$ is simple).

Indeed, we have for $\phi(\omega)=\phi_{*}(\omega)+\delta(\omega)$ :

$$
\begin{aligned}
& \inf _{\phi \in \mathcal{F}} \frac{K}{2}\left[\ln \left(\int \exp \{\phi(\omega)\} p_{\mu}(\omega) \Pi(d \omega)\right)+\ln \left(\int \exp \{-\phi(\omega)\} p_{\nu}(\omega) \Pi(d \omega)\right)\right] \\
& =\inf _{\delta \in \mathcal{F}} \frac{K}{2}\left[\ln \left(\int \exp \left\{\phi_{*}(\omega)+\delta(\omega)\right\} p_{\mu}(\omega) \Pi(d \omega)\right)+\ln \left(\int \exp \left\{-\phi_{*}(\omega)-\delta(\omega)\right\} p_{\nu}(\omega) \Pi(d \omega)\right)\right] \\
& =\inf _{\delta \in \mathcal{F}}[\underbrace{\ln \left(\int \exp \{\delta(\omega)\} \sqrt{p_{\mu}(\omega) p_{\nu}(\omega)} \Pi(d \omega)\right)+\ln \left(\int \exp \{-\delta(\omega)\} \sqrt{p_{\mu}(\omega) p_{\nu}(\omega)} \Pi(d \omega)\right)}_{f(\delta)}]
\end{aligned}
$$

$f(\delta)$ is convex and even function of $\delta \in \mathcal{F}$ and as such achieves its minimum at $\delta=0$. 
- By the above computation,

$$
\begin{aligned}
& \operatorname{Opt}_{i j}(K)=\max _{x \in X_{i}, y \in X_{j}} Q_{i j K}(x, y) \\
& =\max _{x \in X_{i}, y \in X_{j}}\left[\frac{1}{2} g^{T}[y-x]+\inf _{\alpha>0} \alpha\left[K \ln \left(\int \sqrt{p_{A_{i}(x)}(\omega) p_{a_{j}(y)}(\omega)} \sqcap(d \omega)\right)+\ln (2 J / \epsilon)\right]\right] \\
& =\max _{x, y}\left\{\frac{1}{2} g^{T}[y-x]: K \ln \left(\int \sqrt{p_{A_{i}(x)}(\omega) p_{A_{j}(y)}(\omega)} \sqcap(d \omega)\right)+\ln (2 J / \epsilon) \geq 0, x \in X_{i}, y \in X_{j}\right\}
\end{aligned}
$$

- To complete the proof, it suffices to verify that

$$
\frac{K}{M}>\frac{2 \ln (2 J / \epsilon)}{\ln \left([4 \epsilon(1-\epsilon)]^{-1}\right)} \Rightarrow \operatorname{Opt}_{i j}(K) \leq \operatorname{RiskOpt}_{\epsilon}(M) \text { for all } i, j \text {. }
$$

Indeed, assuming the opposite, we can find $i, j$ and $\bar{x} \in X_{i}, \bar{y} \in X_{j}$ such that setting $p=p_{A_{i}(\bar{x})}, q=p_{A_{j}(\bar{y})}$, we have

$$
\begin{gathered}
\frac{1}{2} g^{T}[\bar{y}-\bar{x}]>\operatorname{RiskOpt}_{\epsilon}(M) \\
K \ln \left(\int \sqrt{p(\omega) q(\omega)} \Pi(d \omega)\right)+\ln (2 J / \epsilon) \geq 0
\end{gathered}
$$




$$
\begin{gathered}
\frac{1}{2} g^{T}[\bar{y}-\bar{x}]>\operatorname{RiskOpt}_{\epsilon}(M) \\
K \ln \left(\int \sqrt{p(\omega) q(\omega)} \Pi(d \omega)\right)+\ln (2 J / \epsilon) \geq 0 \text { for some } \bar{x} \in X_{i}, \bar{y} \in X_{j}
\end{gathered}
$$

- (a) implies that simple hypotheses

$$
H_{1}: \omega^{M} \sim p^{M}, \quad H_{2}: \omega^{M} \sim q^{M}
$$

about distribution of $\omega^{M}$ can be decided upon with risk $\leq \epsilon$, whence

$$
\begin{aligned}
& \int \min \left[p^{M}\left(\omega^{M}\right), q^{M}\left(\omega^{M}\right)\right] \Pi^{M}\left(d \omega^{M}\right) \leq 2 \epsilon \\
& \Rightarrow\left[\int \sqrt{p(\omega) q(\omega)} \Pi(d \omega)\right]^{M}=\int \sqrt{p^{M}\left(\omega^{M}\right), q^{M}\left(\omega^{M}\right)} \Pi^{M}\left(d \omega^{M}\right) \\
& =\int \sqrt{\min \left[p^{M}\left(\omega^{M}\right), q^{M}\left(\omega^{M}\right)\right]} \sqrt{\max \left[p^{M}\left(\omega^{M}\right), q^{M}\left(\omega^{M}\right)\right]} \Pi^{M}\left(d \omega^{M}\right) \\
& \quad \leq\left[\int \min \left[p^{M}\left(\omega^{M}\right), q^{M}\left(\omega^{M}\right)\right] \Pi^{M}\left(d \omega^{M}\right)\right]^{1 / 2}\left[\int \max \left[p^{M}\left(\omega^{M}\right), q^{M}\left(\omega^{M}\right)\right] \Pi^{M}\left(d \omega^{M}\right)\right]^{1 / 2} \\
& \quad=\left[\int \min \left[p^{M}\left(\omega^{M}\right), q^{M}\left(\omega^{M}\right)\right] \Pi^{M}\left(d \omega^{M}\right)\right]^{1 / 2}\left[\int \max \left[p^{M}\left(\omega^{M}\right), q^{M}\left(\omega^{M}\right)\right] \Pi^{M}\left(d \omega^{M}\right)\right]^{1 / 2} \\
& =\left[\int \min \left[p^{M}\left(\omega^{M}\right), q^{M}\left(\omega^{M}\right)\right] \Pi^{M}\left(d \omega^{M}\right)\right]^{1 / 2} \\
& \quad \times\left[\int\left[p^{M}\left(\omega^{M}\right)+q^{M}\left(\omega^{M}\right)-\min \left[p^{M}\left(\omega^{M}\right), q^{M}\left(\omega^{M}\right)\right]\right] \Pi^{M}\left(d \omega^{M}\right)\right]^{1 / 2} \\
& \leq \sqrt{2 \epsilon(2-2 \epsilon)} \\
& \Rightarrow\left[\int \sqrt{p(\omega) q(\omega)} \Pi(d \omega)\right]^{K} \leq[2 \sqrt{\epsilon(1-\epsilon)}]^{K / M}<\frac{\epsilon}{2 J}
\end{aligned}
$$

[due to $\frac{K}{M}>\frac{2 \ln (2 J / \epsilon)}{\ln \left([4 \epsilon(1-\epsilon)]^{-1}\right)}$ and $\left.\epsilon<1 / 2\right]$, what contradicts $(b)$. 


\section{Illustration: Estimating Cumulative Distribution from Nosiy Observations}

- Let $X$ being convex and closed subset of $\Delta_{n}^{o}$, and let $\zeta \sim x \in X$ be a discrete random variable on $\{1, \ldots, n\}$,

(i.e. $\left.P(\zeta=i)=x_{i}, i=1, \ldots, n\right)$.

- Let $\omega \in\{1, \ldots, d\}$ be a discrete random variable, $\omega \sim \mu=A x$, where $A \in \mathbb{R}_{++}^{d \times n}$ is a given stochastic matrix.

Typical examples are

- Convolution model $\omega=\zeta+\xi$ where $\xi$ (the noise) is a discrete random variable with known distribution

- Multiplicative noise model $\omega=\xi \zeta$

- Missing observations model

- ...

Our objective is, given a stationary $K$-repeated observation $\omega^{K}=\left[\omega_{1} \ldots \omega_{K}\right]$, estimate values $F_{i}(x)=e_{i}^{T} x:=\sum_{j=1}^{i} x_{j}$ of the c.d.f. of $x$.

Note: We are in the Discrete o.s. and can apply our apparatus to estimate the value of linear functionals $F_{i}(x)$ 
To compute the estimator $\widehat{F}_{i}(\cdot)$ of $F_{i}$ we proceed as follows.

- We find an optimal solution $[\bar{x}, \bar{y}]$ to the problem

$$
\max \left\{\frac{1}{2} e^{T}(y-x): x, y \in X, K \ln \left[\sum_{i=1}^{d} \sqrt{[A x]_{i}[A y]_{i}}\right]+\ln (2 / \epsilon) \geq 0\right\},
$$

same as solving an equivalent conic quadratic problem

$$
\max _{x, y \in X}\left\{\frac{1}{2} e^{T}(y-x): x, y \in X, \sum_{i=1}^{d} \sqrt{[A x]_{i}[A y]_{i}} \geq(2 / \epsilon)^{1 / K}\right\} .
$$

- Let $[\bar{x} ; \bar{y}]$ be an optimal solution to $(*)$. We set

$$
\widehat{F}_{i}\left(\omega^{K}\right)=\phi_{*}^{T} \widehat{p}^{K}+c_{*}, \quad\left[\phi_{*}\right]_{i}=\nu\left(\sum_{i=1}^{M} \sqrt{[A \bar{x}]_{i}[A \bar{y}]_{i}}\right) \ln \sqrt{\frac{[A \bar{y}]_{i}}{[A \bar{x}]_{i}}}, \quad c_{*}=\frac{1}{2} g^{T}(\bar{x}+\bar{y}),
$$

where $\widehat{p}^{K}$ is the empirical distribution of $\omega$ :

$$
\widehat{p}_{i}^{K}=\frac{1}{K} \sum_{k=1}^{K} 1_{\omega_{k}=i}
$$

and $\nu$ is the Lagrange multiplier of the conic constraint.

Theorem Let $\epsilon \in(0,1 / 2]$. The risk of the estimator $\widehat{g}_{*}$ satisfies:

$$
\operatorname{Risk}_{\epsilon}\left[\widehat{F}_{i} \mid X\right] \leq \frac{2 \ln (2 / \epsilon)}{\ln \left([4 \epsilon(1-\epsilon)]^{-1}\right)} \operatorname{RiskOpt}_{\epsilon}(K) . \quad[\rightarrow 2, \text { as } \epsilon \rightarrow+0]
$$




\section{Application: Convolution Model}

Goal: Suppose that r.v. $\eta \sim F_{\eta}$, supp $\eta \subseteq[-3,3], v \sim F_{v}$, with known $F_{v}$. Given $n=100$ i.i.d. observations $Y_{i}$ of a r.v. $\eta+v$, we want to recover $F_{\eta}(t)$ for a given $t \in[-3,3]$.

- We split $[-3,3]$ into $n=60$ equal segments $\delta_{i}$ of length $\delta$, with the "sampled signal" $x_{i}=P\left(\eta \in \triangle_{i}\right)$. On suppose

$$
\frac{x_{i}-2 x_{i-1}+x_{i-2}}{\Delta^{2}} \leq 1
$$

(a "discrete approximation" of the density $f_{\eta}$ of $\eta$ is twice differentiable with $\left.\left|f_{\eta}^{\prime \prime}(t)\right| \leq 1\right)$.

- We cut the domaine of $Y$ into $d=42$ chunks $\gamma_{i}$ (40 equal segments between ] $Y_{\min }, Y_{\max }$ [ and two "complements" in $\mathbb{R}$. The distribution of $\eta+v$ is approximated with $\mu=A x$, where

$$
A_{i j}=\delta_{i}^{-1} \int_{v \in \gamma_{i}} \int_{u \in \delta_{j}} f_{\xi}(v-u) d v d u .
$$


Numerical illustration: Estimation of $F_{\eta}(0)$, normal nuisance $v, n=100$ observations, $\mathrm{e}=0.05$.

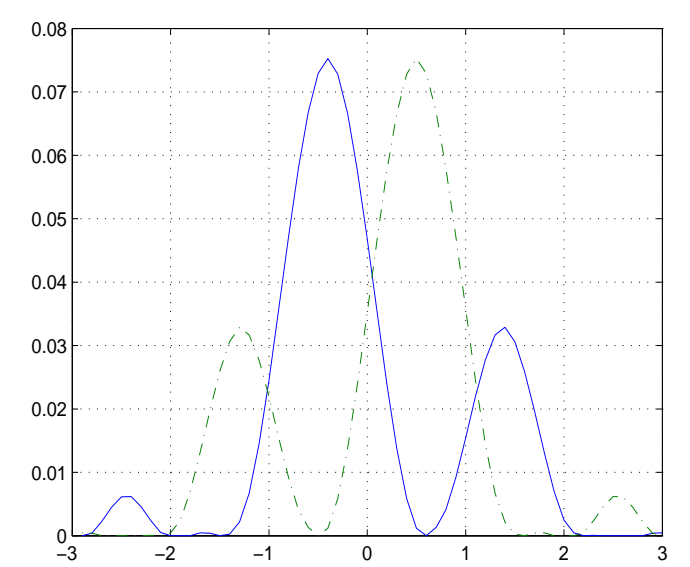

Optimal signals $\bar{x}$ et $\bar{y}$

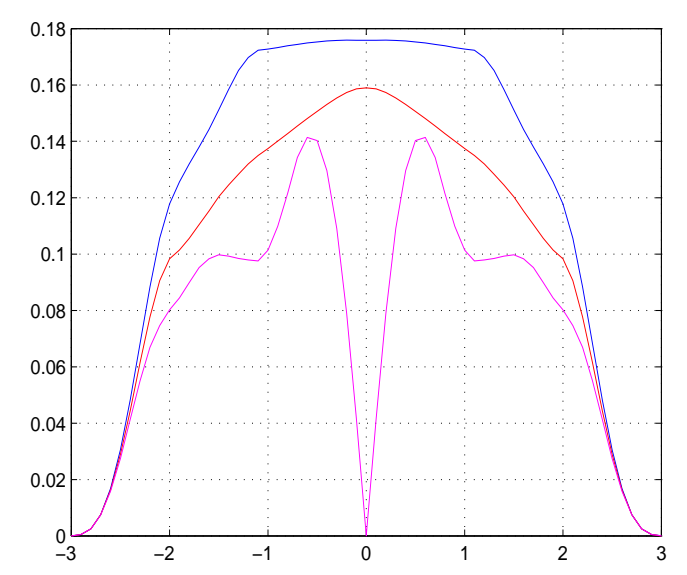

— risk of minmax affine estimator

- constrained to $\mathbf{E}\{\eta\}=0$ risk

- symmetry constrained risk

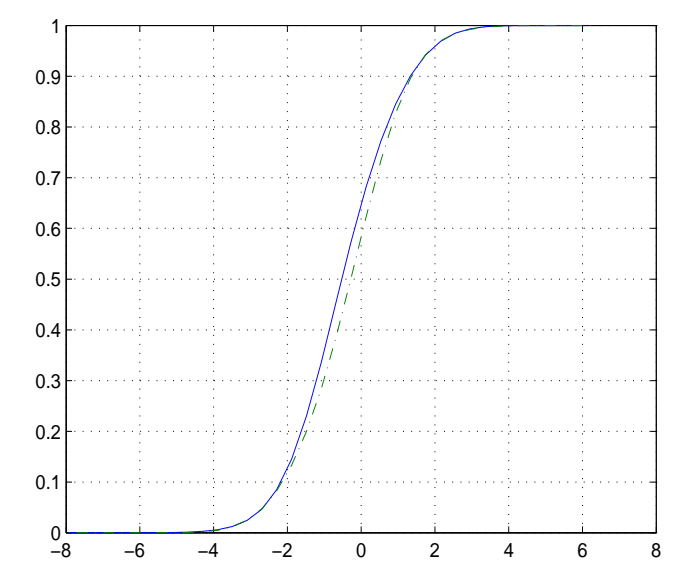

Corresponding c.d.f. of $A \bar{x}$ and of $A \bar{y}$

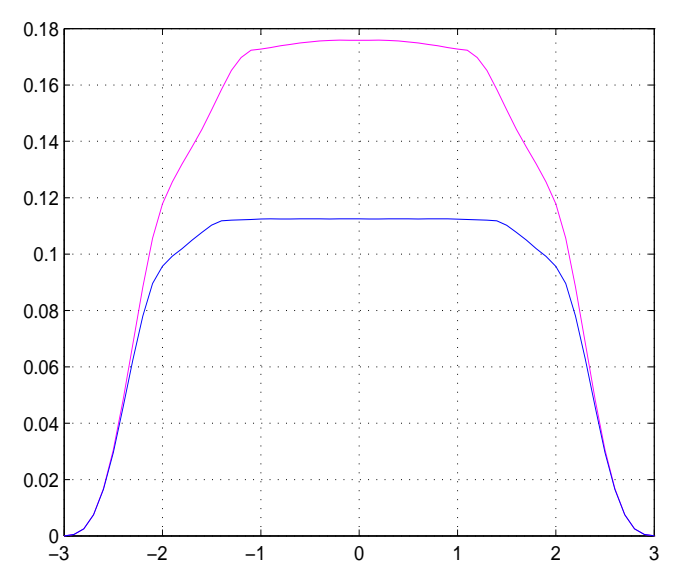

- Gaussian noise $v$

- Laplace noise $v$ 


\section{Sequential Hypothesis Testing}

Motivating example: Election Polls. Population-wide elections with $L$ candidates are to be held.

Preferences are represented by $L$-dimensional basic orth

$$
[0 ; \ldots ; 0 ; 1 ; 0 ; \ldots ; 0]
$$

with 1 in position $\ell$ meaning the vote is for candidate \# $\ell$.

Equivalently: Preference $\omega$ of a voter is a vertex in the probabilistic simplex

$$
\Delta_{L}=\left\{p \in \mathbb{R}^{L}: p \geq 0, \sum_{\ell} p_{\ell}=1\right\} .
$$

- The average $\mu=\left[\mu_{1} ; \ldots ; \mu_{L}\right]$ of preferences of all voters "encodes" election's outcome: $\mu_{\ell}$ is the fraction of voters supporting $\ell$-th candidate, and the winner corresponds to the largest entry in $\mu$ (assumed to be uniquely defined).

$\mu$ is a probabilistic vector: $\mu \in \Delta_{L}$. We think of $\mu$ as of a probability distribution on the $L$-element set $\Omega=\operatorname{Ext}\left(\Delta_{L}\right)$ of vertices of $\Delta_{L}$. 
Our goal is to design election poll - to select $K$ voters at random from the uniform over the population distribution and to observe their preferences, in order to predict, with reliability $1-\epsilon$, election's outcome.

The model is drawing a stationary $K$-repeated observation $\omega^{K}=\left(\omega_{1}, \ldots, \omega_{K}\right)$, $\omega_{k} \in \Omega$, from distribution $\mu$.

Assume once for ever that the elections never end with "near tie," that is, the fraction of votes for the winner is at least by a known margin $\delta$ larger than the fraction of votes for every no-winner.

We introduce $L$ hypotheses on the distribution $\mu$ of $\omega_{1}, \ldots, \omega_{K}$ :

$$
H_{\ell}: \mu \in \mathcal{P}_{\ell}=\left\{\mu \in \Delta_{L}: l \mu_{\ell} \geq \mu_{\ell^{\prime}}+\delta, \forall \ell^{\prime} \neq \ell\right\}, \quad \ell=1, \ldots, L
$$

- Our goal is to specify $K$ in a way which allows to decide on $H_{1}, \ldots, H_{L}$ via stationary $K$-repeated observations with risk $\leq \epsilon$.

- We are in the case of Discrete o.s., and can use our machinery to build a near-optimal $K$-observation test deciding on $H_{1}, \ldots, H_{L}$ up to trivial closeness

$\mathcal{C}$ : " $H_{\ell}$ is close to $H_{\ell^{\prime}}$ iff $\ell=\ell^{\prime \prime}$ "

and select the smallest $K$ for which the $\mathcal{C}$-risk of this test is $\leq \epsilon$. 
Illustration $L=2$ : In this case $\Omega$ is a two-point set of basic orths in $\mathbb{R}^{2}$, the minimum risk single-observation detector is

$$
\phi_{*}(\omega)=\frac{1}{2} \ln \left(\frac{1+\delta}{1-\delta}\right)\left[\omega^{1}-\omega^{2}\right]: \Omega \rightarrow \mathbb{R}
$$

and $\operatorname{Risk}\left[\phi_{*} \mid \mathcal{P}_{1}, \mathcal{P}_{2}\right]=1-\delta^{2}$

$$
\begin{aligned}
& \Rightarrow K=\text { Ceil }\left(\frac{\ln (1 / \epsilon)}{\ln \left(1 /\left(1-\delta^{2}\right)\right)}\right) \asymp \frac{1}{\delta^{2}} \ln (1 / \epsilon) . \\
& \begin{array}{c|c|c|c|c|}
\delta & 0.3162 & 0.1000 & 0.0316 & 0.0100 \\
\hline \underline{K}, K & 51,88 & 534,917 & 5379,9206 & 53820,92064
\end{array} \\
& \underline{K} \text { : lower bound on optimal poll size, } \epsilon=.01
\end{aligned}
$$

USA Presidential Elections-2016:

\begin{tabular}{||c||c||c|c||}
\hline \hline State & $\begin{array}{c}\text { Actual } \\
\text { margin }\end{array}$ & $\begin{array}{c}\text { Poll size, } \\
\text { lower bound }\end{array}$ & $\begin{array}{c}\text { Poll size, } \\
\text { upper bound }\end{array}$ \\
\hline \hline Wisconsin & 0.0041 & 24576 & 88663 \\
\hline Pennsylvania & 0.0038 & 28978 & 104545 \\
\hline Michigan & 0.0012 & 281958 & 1017227 \\
\hline \hline
\end{tabular}

Confidence level 95\% 


\begin{tabular}{c|c|c|c|c|}
$\delta$ & 0.3162 & 0.1000 & 0.0316 & 0.0100 \\
\hline$\underline{K}, K$ & 51,88 & 534,917 & 5379,9206 & 53820,92064 \\
\multicolumn{5}{c}{ : lower bound on optimal poll size, $\epsilon=.01$}
\end{tabular}

Required size of election poll grows rapidly as "winning margin" decreases.

Question: Can we do better?

Partial remedy: Let us pass to sequential tests, where we attempt to make conclusion before all $K$ respondents required by the worst-case-oriented analysis are interviewed.

If elections are about to be "landslide" (i.e., in unknown to us actual distribution $\mu_{*}$ of voters' preferences the winner beats all other candidates by margin $\delta_{*} \gg \delta$ ), the winner hopefully can be identified after a relatively small number of interviews. 
Strategy. We select a number $S$ of valuations and associate with valuation $s$ number $K(s)$ of observations, $K(1)<\ldots<K(S)$.

- the $s$-th valuation is complete when $K(s)$ observations are collected; then, we apply to the collected so far observation $\omega^{K(s)}=\left(\omega_{1}, \ldots, \omega_{K(s)}\right)$ a test $\mathcal{T}_{s}$ which, depending on $\omega^{K(s)}$,

- either accepts exactly one of the hypotheses $H_{1}, \ldots, H_{L}$, in which case we terminate,

- or claims that information collected so far is not sufficient to decide, in which case we either collect more observations (when $s<S$ ) or terminate (when $s=S$ ).

Specifications: We want the overall procedure to be

- conclusive: a decision should be made during one of the $S$ valuations (thus, inference should be guaranteed when valuation $S$ is reached);

- reliable: whenever the true distribution $\mu_{*}$ underlying observations obeys one of our $L$ hypotheses, the $\mu_{*}$-probability for this hypothesis to be accepted should be $\geq 1-\epsilon$, where $\epsilon \in(0,1)$ is a given in advance risk bound. 
Implementation: We select somehow the number of valuations $S$ and set $\delta_{s}=\delta^{s / S}$ so that $\delta_{1}>\delta_{2}>\ldots>\delta_{S}=\delta$; we split risk bound $\epsilon$ into $S$ parts $\epsilon_{s}: \epsilon_{s}>0, s \leq S \& \sum_{s=1}^{S} \epsilon_{s}=\epsilon$;

- For $s<S$, we define $2 L$ hypotheses

$$
\begin{aligned}
& H_{2 \ell-1}^{s}= \begin{array}{l}
H_{\ell}: \mu \in \mathcal{P}_{2 \ell-1}^{s}=\mathcal{P}_{\ell}:=\left\{\mu \in \Delta_{L}: \mu_{\ell} \geq \delta_{S}+\max _{\ell^{\prime} \neq \ell} \mu_{\ell^{\prime}}\right\} \\
\text { "weak hypothesis" }
\end{array} \\
& H_{2 \ell}^{s}=\begin{array}{l}
\left\{\mu \in \mathcal{P}_{2 \ell}^{s}:=\left\{\mu \in \Delta_{L}: \mu_{\ell} \geq \delta_{s}+\max _{\ell^{\prime} \neq \ell} \mu_{\ell^{\prime}}\right\} \subset \mathcal{P}_{\ell}\right\} \\
\text { "strong hypothesis" }
\end{array}
\end{aligned}
$$

$1 \leq \ell \leq L$, and assign $H_{2 \ell-1}^{s}$ and $H_{2 \ell}^{s}$ with color $\ell, 1 \leq \ell \leq L$.

- For $s=S$ we consider $L$ hypotheses $H_{\ell}^{S}=H_{\ell}, \ell=1, \ldots, L$, with $H_{\ell}^{S}$ assigned color $\ell$.

- For $s<S$, we introduce closeness relation $\mathcal{C}_{s}$ on the collection of hypotheses $H_{1}^{s}, \ldots, H_{2 L}^{s}$ as follows:

- the only hypotheses close to a strong hypothesis $H_{2 \ell}^{s}$ are the hypotheses $H_{2 \ell}^{s}$ and $H_{2 \ell-1}^{s}$ of the same color;

- the only hypotheses close to a weak hypothesis $H_{2 \ell-1}^{s}$ are all weak hypotheses and the strong hypothesis $H_{2 \ell}$ of the same color as $H_{2 \ell-1}$.

- For $s=S$, the $\mathcal{C}_{s^{-}}$-closeness is trivial: $H_{\ell}^{S} \equiv H_{\ell}$ is $\mathcal{C}_{S^{-}}$-close to $H_{\ell^{\prime}}^{S} \equiv H_{\ell^{\prime}}$ if and only if $\ell=\ell^{\prime}$. 


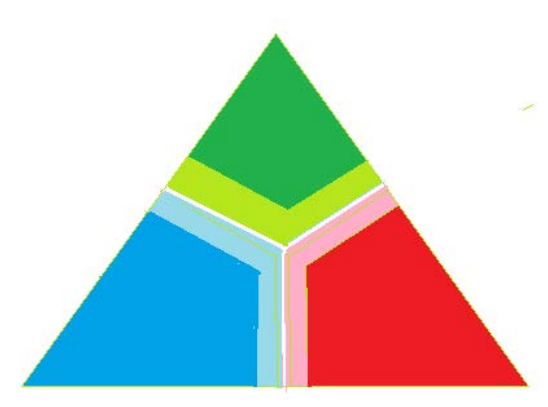

\section{3-candidate hypotheses in the probabilistic simplex $\Delta_{3}$}

[weak green] $\quad M_{1}$ dark green + light green: candidate A wins with margin $\geq \delta_{S}$

[strong green] $\quad M_{1}^{s}$ dark green: candidate A wins with margin $\geq \delta_{s}>\delta_{S}$

[weak red]

[strong red]

$M_{2} \quad$ dark red + pink: candidate B wins with margin $\geq \delta_{S}$

[weak blue]

$M_{2}^{s}$

dark red: candidate $\mathrm{B}$ wins with margin $\geq \delta_{s}>\delta_{S}$

[strong blue]

$M_{3}$

dark blue + light blue: candidate $\mathrm{C}$ wins with margin $\geq \delta_{S}$

$M_{3}^{s} \quad$ dark blue: candidate $\mathrm{C}$ wins with margin $\geq \delta_{s}>\delta_{S}$

- $H_{2 \ell-1}^{s}: \mu \in M_{\ell}$ [weak hypothesis]

weak hypothesis $H_{2 \ell-1}^{s}$ is $\mathcal{C}_{s}$-close to itself, to all other weak hypotheses and to strong hypothesis $H_{2 \ell}^{s}$ of the same color as $H_{2 \ell-1}^{s}$

- $H_{2 \ell}^{s}: \mu \in M_{\ell}^{s}$ [strong hypothesis]

strong hypothesis $H_{2 \ell}^{s}$ is $\mathcal{S}$-close only to itself and to weak hypothesis $H_{2 \ell-1}^{s}$ of the same color as $H_{2 \ell-1}^{s}$ 
Note: We are in the case of stationary repeated observations in Discrete o.s., and the hypotheses $H_{j}^{s}$ are of the form

"i.i.d. observations $\omega_{1}, \omega_{2}, \ldots$ are drawn from distribution $\mu \in M_{j}^{s}$ with nonempty closed convex sets $M_{j}^{s} \subset \Delta_{L}$ ", and sets $M_{j}^{s}, M_{j^{\prime}}^{s}$ with $\left(j, j^{\prime}\right) \notin \mathcal{C}_{s}$ do not intersect

$\Rightarrow$ risks of the optimal pairwise detectors for $\mathcal{P}_{j}^{s}, \mathcal{P}_{j^{\prime}}^{s},\left(j, j^{\prime}\right) \notin \mathcal{C}_{s}$, are $<1$

$\Rightarrow$ we can efficiently find the smallest $K=K(s)$ for which a test $\mathcal{T}=\mathcal{T}_{s}$ deciding, via stationary $K(s)$-repeated observations, on the hypotheses $\left\{H_{j}^{s}\right\}_{j}$ has $\mathcal{C}_{s}$-risk $\leq \epsilon_{s}$. 
Our inference routine works as follows:

- we observe $\omega_{k}, k=1,2, \ldots, K(S)$ (i.e., carry interviews with one by one randomly selected voters), and perform $s$-th valuation to make conclusion when $K(s)$ observations are acquired $(K(s)$ interviews are completed).

- At $s$-th valuation, we apply the test $\mathcal{T}_{s}$ to observation $\omega^{K(s)}$. If the test does accept some of the hypotheses $H_{j}^{s}$ and all accepted hypotheses have the same color $\ell$, we accept $\ell$-th of our original hypotheses $H_{1}, \ldots, H_{L}$ (i.e., predict that $\ell$-th candidate will be the winner) and terminate,

- otherwise we proceed to next observation round (i.e., next interviews) (when $s<S$ ) or claim the winner to be, say, the first candidate and terminate (when $s=S)$. 
- The risk of the outlined sequential hypothesis testing procedure is $\leq \epsilon$ : whenever the distribution $\mu_{*}$ underlying observations obeys hypothesis $H_{\ell}$ for some $\ell \leq L$, the $\mu_{*}$-probability of the event " $H_{\ell}$ is the only accepted hypothesis" is at least $1-\epsilon$.

- The worst-case volume of observations $K(S)$ is within logarithmic factor from the minimal number of observations allowing to decide on the hypotheses $H_{1}, \ldots, H_{L}$ with risk $\leq \epsilon$.

- Whenever the distribution $\mu_{*}$ underlying observations obeys strong hypothesis $H_{2 \ell}^{s}$ for some $\ell$ and $s$ ('distribution $\mu_{*}$ of voters' preferences corresponds to winning margin at least $\delta_{s}$ ), the conclusion, with $\mu_{*}$ probability $\geq 1-\epsilon$, will be made in course of the first $s$ valuations (i.e., in course of the first $K(s)$ interviews).

Informally: In "landslide elections," the winner will be predicted reliably after a small number of interviews. 


\section{Numerical Illustration: 2-Candidate Elections}

\section{Setup:}

- \# of candidates $L=2$

- $\delta_{s}=10^{-s / 4}$

- range of \# of valuations $S: 1 \leq S \leq 8$

\section{Numerical Results:}

\begin{tabular}{||c||c|c|c|c|c|c||}
\hline \hline$S$ & 1 & 2 & 4 & 5 & 6 & 8 \\
\hline$\delta=\delta_{S}$ & 0.5623 & 0.3162 & 0.1000 & 0.0562 & 0.0316 & 0.0100 \\
\hline \hline$K$ & 25 & 88 & 287 & 917 & 9206 & 92098 \\
\hline$K(S)$ & 25 & 152 & 1594 & 5056 & 16005 & 160118 \\
\hline \hline
\end{tabular}

Volume $K$ of non-sequential test, number of valuations $S$ and worst-case volume $K(S)$ of sequential test as functions of winning margin $\delta=\delta_{S}$. Risk $\epsilon$ is set to 0.01 .

Note that worst-case volume of sequential test is essentially worse than the volume of nonsequential test.

But: When drawing the true distribution $\mu_{*}$ of voters' preferences at random from the uniform distribution on the set of $\mu$ 's with winning margin $\geq 0.01$, the typical size of observations used by Sequential test with $S=8$ prior to termination is $\ll K(S)$ :

Empirical Volume of Sequential test

\begin{tabular}{|c|c|c|c|c|c|c|c|c|c|}
\hline median & mean & $60 \%$ & $65 \%$ & $75 \%$ & $80 \%$ & $85 \%$ & $90 \%$ & $95 \%$ & $100 \%$ \\
\hline \hline 177 & 9182 & 177 & 397 & 617 & 1223 & 1829 & 8766 & 87911 & 160118 \\
\hline
\end{tabular}

Column "X\%": empirical X\%-quantile of test's volume. Data over 1,000 experiments.

Empirical risk: 0.01 


\section{Measurement Design}

Observation: In our Hypothesis Testing setup, observation scheme is our "environment" and is completely out of our control. However, there are situations where the observation scheme is under our partial control.

Example: Election Poll revisited. In our original problem, a particular voter was represented by basic orth $\omega=[0 ; \ldots ; 0 ; 1 ; 0 ; \ldots ; 0] \in \mathbb{R}^{L}$, with entry 1 in position $\ell$ meaning that the voter prefers candidate $\ell$ to all other candidates. Our goal was to predict the winner by observing preferences of respondents selected at random from uniform distribution on voters' population.

A modification: Imagine we can split voters in $I$ non-intersecting groups (say, according to age, education, gender, income, occupation,...) in such a way that we have certain a priori knowledge of the distributions of preferences within the group. 
In this situation, our poll can be reorganized:

- We assign groups with nonnegative weights $q_{i}$ summing up to 1

- To organize an interview, we first select at random one of the groups, with probability $q_{i}$ to select group $i$, and then select a respondent from $i$-th group at random, from uniform distribution on the group.

Note: When $q_{i}$ is equal to the fraction $\theta_{i}$ of group $i$ in the entire population, the above policy reduces to the initial one. It can make sense, however, to use $q_{i}$ different from $\theta_{i}$, with $q_{i} \ll \theta_{i}$ if a priori information about preferences of voters from $i$-th group is rich, and $q_{i} \gg \theta_{i}$ if this information is poor. Hopefully, this will allow us to make more reliable predictions with the same total number of interviews. 
The model of outlined situation is as follows:

- We characterize distribution of preferences within group $i$ by vector $\mu^{i} \in \Delta_{L}$. for $1 \leq j \leq L, j$-th entry in $\mu^{i}$ is the fraction of voters in group $i$ voting for candidate $j$;

The population-wide distribution of voters' preferences is $\mu=\sum_{i=1}^{I} \theta_{i} \mu^{i}$.

- A priori information on distribution of preferences of voters from group $i$ is modeled as the inclusion $\mu^{i} \in M^{i}$, for some known subset $M^{i} \subset \Delta_{L}$ which we assume to be nonempty convex compact set.

- Output of particular interview is pair $(i, j)$, where $i \in\{1, \ldots, I\}$ is selected at random according to probability distribution $q$, and $j$ is the candidate preferred by respondent selected from group $i$ at random, according to uniform distribution on the group. 
Observation (outcome of an interview) becomes

$$
\begin{gathered}
\omega:=(i, j) \in \Omega=\{1, \ldots, I\} \times\{1, \ldots, L\}, \\
\quad \operatorname{Prob}\{\omega=(i, j)\}=p(i, j):=q_{i} \mu_{j}^{i} .
\end{gathered}
$$

Hypotheses to decide upon are

$$
H_{\ell}[q]: p \in \mathcal{P}_{\ell}[q]:=\left\{\left\{p_{i j}=q_{i} \mu_{j}^{i}\right\}_{\substack{1 \leq i \leq I, 1 \leq j \leq L}}: \begin{array}{l}
\mu^{i} \in M^{i} \forall i, \\
{\left[\sum_{i} \theta_{i} \mu^{i}\right]_{\ell} \geq \delta+\left[\sum_{i} \theta_{i} \mu^{i}\right]_{\ell^{\prime}} \forall\left(\ell^{\prime} \neq \ell\right)}
\end{array}\right\}
$$

$H_{\ell}[q], \ell=1, \ldots, L$, states that the "signal" $\mu=\left[\mu^{1} ; \ldots ; \mu^{I}\right]$ underlying distribution $p$ of observations $\omega$ induces population-wide distribution $\sum_{i} \theta_{i} \mu^{i}$ of votes resulting in electing candidate $\ell$ with winning margin $\geq \delta$. 


$$
H_{\ell}[q]: p \in \mathcal{P}_{\ell}[q]:=\left\{\left\{p_{i j}=q_{i} i_{j}^{i}\right\}_{1 \leq 1 \leq 1}: \begin{array}{l}
\mu^{i} \in M^{i} \forall i t i \\
{\left[\sum_{i} \theta_{i} \mu^{i}\right]_{\ell} \geq \delta+\left[\sum_{i} \theta_{i} \mu^{i}\right]_{\ell^{\prime}} \forall\left(\ell^{\prime} \neq \ell\right)}
\end{array}\right\}
$$

Hypotheses $H_{\ell}[q]$ are of the form

$$
\begin{gathered}
H_{\ell}[q]=\left\{p=A[q] \mu: \mu:=\left[\mu^{1} ; \ldots ; \mu^{L}\right] \in \mathcal{M}^{\ell}\right\}, \\
{[A[q] \boldsymbol{\mu}]_{i j}=q_{i} \mu_{j}^{i},}
\end{gathered}
$$

where $\mathcal{M}^{\ell}, \ell=1, \ldots, L$, are nonempty nonintersecting convex compact subsets in $\underbrace{\Delta_{L} \times \ldots \times \Delta_{L}}_{I}$

Note: Election Poll with $K$ interviews corresponds to stationary $K$-repeated observation in Discrete o.s. with $I L$-element observation space $\Omega$

$\Rightarrow$ Given $K$, we can design a near-optimal detector-based test $\mathcal{T}_{K}$ deciding via stationary $K$-repeated observation (i.e., via the outcomes of $K$ interviews) on hypotheses $H_{\ell}[q], \ell=1, \ldots, L$ up to trivial closeness

$$
H_{\ell}[q] \text { is close to } H_{\ell^{\prime}}[q] \text { iff } \ell=\ell^{\prime} \text {. }
$$

This test will predict the winner with reliability $1-\operatorname{Risk}\left[\mathcal{T}_{K} \mid H_{1}[q], \ldots, H_{L}[q]\right]$. 


$$
\begin{gathered}
H_{\ell}[q]=\left\{p=A[q] \boldsymbol{\mu}: \boldsymbol{\mu}:=\left[\mu^{1} ; \ldots ; \mu^{L}\right] \in \mathcal{M}^{\ell}\right\}, \\
{[A[q] \boldsymbol{\mu}]_{i j}=q_{i} \mu_{j}^{i},}
\end{gathered}
$$

Setting $\chi_{\ell \ell^{\prime}}=\left\{\begin{array}{ll}0, & \ell=\ell^{\prime} \\ 1, & \ell \neq \ell^{\prime}\end{array}\right.$, we get

$$
\operatorname{Risk}\left[\mathcal{T}_{K} \mid H_{1}[q], \ldots, H_{L}[q]\right] \leq \epsilon_{K}[q]:=\left\|\left[\epsilon_{\ell \ell^{\prime}}^{K}[q] \chi_{\ell \ell^{\prime}}\right]_{\ell, \ell^{\prime}=1}^{L}\right\|_{2,2},
$$

where

$$
\begin{aligned}
\epsilon_{\ell \ell^{\prime}}[q] & =\max _{\boldsymbol{\mu} \in \mathcal{M}^{\ell}, \boldsymbol{\nu} \in \mathcal{M}^{\ell^{\prime}}} \sum_{i, j} \sqrt{[A[q] \boldsymbol{\mu}]_{i j}[A[q] \boldsymbol{\nu}]_{i j}} \\
& =\max _{\boldsymbol{\mu} \in \mathcal{M}^{\ell}, \boldsymbol{\nu} \in \mathcal{M}^{\ell^{\prime}}} \underbrace{\sum_{i=1}^{I} q_{i}\left[\sum_{j=1}^{L} \sqrt{\mu_{j}^{i} \nu_{j}^{i}}\right]}_{\Phi(q ; \boldsymbol{\mu}, \boldsymbol{\nu})}
\end{aligned}
$$

Observe that $\Phi(q ; \boldsymbol{\mu}, \boldsymbol{\nu})$ is linear in $q$. 
Let us carry out Measurement Design - optimize the value $\epsilon_{K}[q]$ in $q$.

Main observation: $\epsilon_{K}[q]=\Gamma(\Psi(q))$, where

- $\Gamma(Q)=\left\|\left[\left(Q_{\ell \ell^{\prime}}\right)^{K} \chi_{\ell \ell^{\prime}}\right]_{\ell, \ell^{\prime}=1}^{L}\right\|_{2,2}$ is efficiently computable convex and entrywise nondecreasing function on the space of nonnegative $L \times L$ matrices

- $\Psi(q)$ is matrix-valued function with efficiently computable convex in $q$ and nonnegative entries

$$
\Psi_{\ell \ell^{\prime}}(q)=\max _{\boldsymbol{\mu} \in \mathcal{M}^{\ell}, \boldsymbol{\nu} \in \mathcal{M}^{\ell^{\prime}}} \Phi(q ; \boldsymbol{\mu}, \boldsymbol{\nu})
$$

$\Rightarrow$ Optimal selection of $q_{i}$ 's reduces to solving explicit convex problem

$$
\min _{q}\left\{\Gamma(\Psi(q)): q=\left[q_{1} ; \ldots ; q_{I}\right] \geq 0, \sum_{i=1}^{I} q_{i}=1\right\}
$$




\section{How it Works: Measurement Design in Election Polls}

\section{Setup:}

- Election Poll problem with $L$ candidates and winning margin $\delta=0.05$

- Reliability $\epsilon=0.01$

- A priori information on voters' preferences in groups:

$$
M^{i}=\left\{\mu^{i} \in \Delta_{L}: p_{\ell}^{i}-u_{i} \leq \mu_{\ell}^{i} \leq p_{\ell}^{i}+u_{i}, \ell \leq L\right\}
$$

- $p^{i}$ : randomly selected probabilistic vector $\bullet u_{i}$ : uncertainty level

\section{Sample of results:}

\begin{tabular}{||c|c||c||c||c|c||}
\hline \hline$L$ & $I$ & $\begin{array}{c}\text { Group sizes } \theta \\
\text { Uncertainty levels } u\end{array}$ & $K_{\text {ini }}$ & $q_{\text {Opt }}$ & $K_{\text {Opt }}$ \\
\hline \hline 2 & 2 & $\begin{array}{c}\theta=[0.50 ; 0.50] \\
u=[0.03 ; 1.00]\end{array}$ & 1212 & {$[0.44 ; 0.56]$} & 1194 \\
\hline 2 & 2 & $\begin{array}{c}{[0.50 ; 0.50]} \\
{[0.02 ; 1.00]}\end{array}$ & 2699 & {$[0.00 ; 1.00]$} & 1948 \\
\hline 3 & 3 & $\begin{array}{c}{[0.33 ; 0.33 ; 0.33]} \\
{[0.02 ; 0.03 ; 1.00]}\end{array}$ & 3177 & {$[0.00 ; 0.46 ; 0.54]$} & 2726 \\
\hline 5 & 4 & $\begin{array}{c}{[0.25 ; 0.25 ; 0.25 ; 0.25]} \\
{[0.02 ; 0.02 ; 0.03 ; 1.00]}\end{array}$ & 2556 & {$[0.00 ; 0.13 ; 0.32 ; 0.55]$} & 2086 \\
\hline 5 & 4 & $\begin{array}{c}0.25 ; 0.25 ; 0.25 ; 0.25] \\
{[1.00 ; 1.00 ; 1.00 ; 1.00]}\end{array}$ & 4788 & {$[0.25 ; 0.25 ; 0.25 ; 0.25]$} & 4788 \\
\hline \hline
\end{tabular}

Effect of measurement design. $K_{\text {ini }}$ and $K_{\text {opt }}$ are the poll sizes required for 0.99 -reliable prediction of the winner when $q_{i}=\theta_{i}$ and $q=q_{\mathrm{Opt}}$, respectively. Note: Uncertainty $=1.00 \Leftrightarrow$ No a priori information 
- In numerous situations, we do have partial control of observation scheme and thus can look for optimal Measurement Design.

- However, situations where optimal Measurement Design can be found efficiently, like in design of Election Polls, are rare.

- Two other examples of such situation are Poisson o.s. and Gaussian o.s. with time control. 


\section{Poisson 0.s. with time control.}

Typical models where Poisson o.s. arises are as follows:

- there exists a "signal" $x$ known to belong to some convex compact set $\subset \mathbb{R}^{n}$

For example, in Positron Emission Tomography, $x$ is (discretized) density of radioactive tracer administered to patient

- We observe random vector $\omega \in \mathbb{R}^{m}$ with independent entries $\omega_{i} \sim$ Poisson $\left(a_{i}^{T} x\right)$, and want to make inferences on $x$.

In PET, patient is injected radioactive tracer which concentrates in areas we are interested in. Tracer disintegrates, and every disintegration act results in pair of gamma-quants flying in opposite directions along a randomly oriented line passing through disintegration point. This line is registered when two detector cells are (nearly) simultaneously hit:

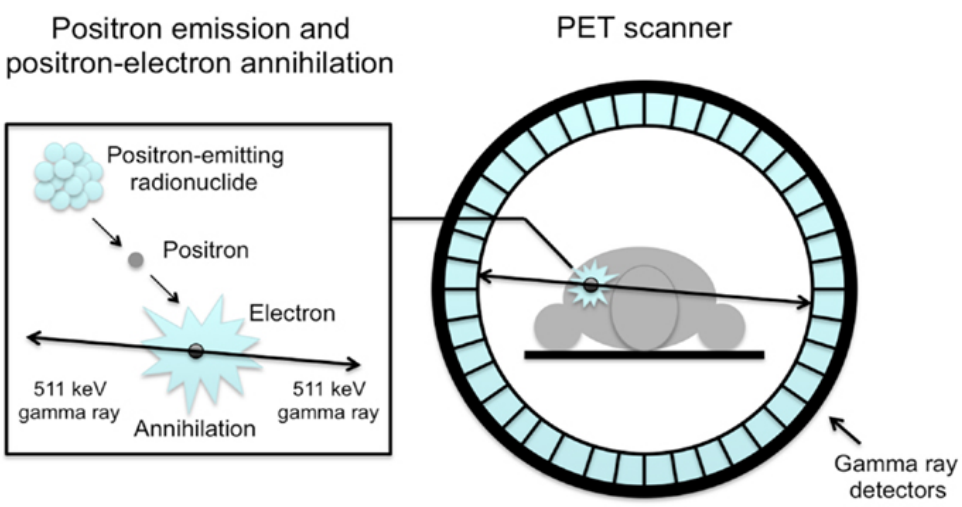


The data acquired in PET study are the numbers $\omega_{i}$ of lines registered in bins (pairs of detector cells) $i=1, \ldots, m$ over a time horizon $T$. We have

$$
\omega_{i} \sim \operatorname{Poisson}\left(T \sum_{j=1}^{n} p_{i j} x_{j}\right)
$$

$p_{i j}$ : probability for line emanating from voxel $j=1, \ldots, n$
to cross pair $i=1, \ldots, m$ of detector cells

$$
\Rightarrow A=T\left[p_{i j}\right]_{i \leq m, j \leq n}
$$

In some situations, the sensing matrix $A$ can be partially controlled:

$$
A=A[q]:=\operatorname{Diag}\{q\} A_{*}
$$

- $A_{*}$ : given $m \times n$ matrix; $\bullet q \in \mathcal{Q}$ : vector of control parameters. 
For instance, in a whole body PET scan the position of the patient w.r.t. the scanner is updated several times to cover the entire body.

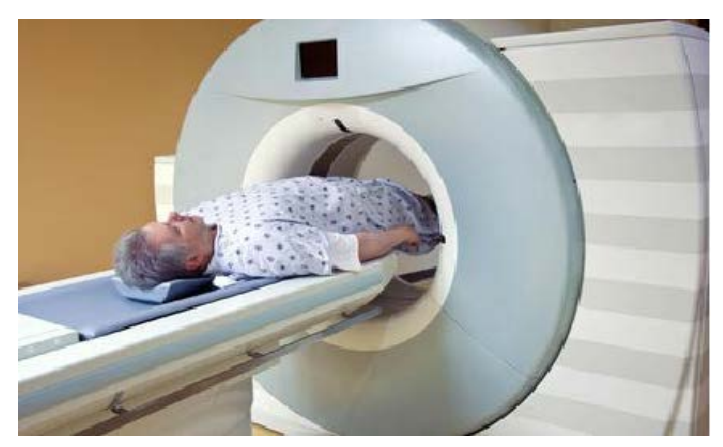

The data acquired in position $\iota$ form subvector $\omega^{\iota}$ in the entire observation $\omega=\left[\omega^{1} ; \ldots ; \omega^{I}\right]$ :

$$
\begin{aligned}
& \omega_{i}^{\iota} \sim \text { Poisson }\left(\left[t_{\iota} A^{\iota} x\right]_{i}\right), 1 \leq i \leq \bar{m}=m / I \\
& {\left[A^{\iota}: \text { given matrices; } t_{\iota}: \text { duration of study in position } \iota\right]}
\end{aligned}
$$

implying that $A=\operatorname{Diag}\{q\} A_{*}$ with properly selected $A_{*}$ and $q$ of the form

$$
q=[\underbrace{t_{1} ; \ldots ; t_{1}}_{\bar{m}} ; \ldots ; \underbrace{t_{I} ; \ldots ; t_{I}}_{\bar{m}}]
$$




$$
\begin{gathered}
H_{\ell}^{q}: \omega_{i} \sim \operatorname{Poisson}\left([A[q] x]_{i}\right) \text { are independent across } i \leq m \text { and } x \in X_{\ell} \\
A=A[q]:=\operatorname{Diag}\{q\} A_{*} \\
\text { • } A_{*} \text { : given } m \times n \text { matrix; } q \in \mathcal{Q}: \text { control parameters. }
\end{gathered}
$$

Our goal is to decide, up to a given closeness $\mathcal{C}$, on $L$ hypotheses on the distribution of Poisson observation $\omega$ :

$$
\begin{aligned}
& H_{\ell}^{q}: \omega \sim \text { Poisson }\left([A[q] x]_{1}\right) \times \ldots \times \text { Poisson }\left([A[q] x]_{m}\right) \\
& \quad x \in X_{\ell}, \quad X_{\ell}: \text { given convex compact sets, } 1 \leq \ell \leq L .
\end{aligned}
$$

- By our theory, the (upper bound on the) $\mathcal{C}$-risk of near-optimal test deciding on $H_{\ell}^{q}, \ell=$ $1, \ldots, L$, is

$$
\begin{gathered}
\epsilon(q)=\left\|\left[\exp \left\{\operatorname{Opt}_{\ell \ell^{\prime}}(q)\right\} \chi_{\ell \ell^{\prime}}\right]_{\ell, \ell^{\prime}=1}^{L}\right\|_{2,2} \\
{\left[\chi_{\ell \ell^{\prime}}=\left\{\begin{array}{ll}
0, & \left(\ell, \ell^{\prime}\right) \in \mathcal{C} \\
1, & \left(\ell, \ell^{\prime}\right) \notin \mathcal{C}
\end{array}, \operatorname{Opt}_{\ell \ell^{\prime}}(q)=\max _{u \in X_{\ell}, v \in X_{\ell^{\prime}}}-\frac{1}{2} \sum_{i=1}^{m}\left(\sqrt{[A[q] u]_{i}}-\sqrt{[A[q] v]_{i}}\right)^{2}\right]\right.}
\end{gathered}
$$

Similarly to the Election Polls, $\epsilon(q)=\Gamma(\Psi(q))$, where

- $\Gamma(Q)=\left\|\left[\exp \left\{Q_{\ell \ell^{\prime}}\right\} \chi_{\ell \ell^{\prime}}\right]_{\ell, \ell^{\prime}=1}^{L}\right\|_{2,2}$ is a convex entrywise nondecreasing function of $L \times L$ matrix $Q$

- $[\Psi(q)]_{\ell \ell^{\prime}}=\exp \left\{\max _{u \in X_{\ell}, v \in X_{\ell^{\prime}}} \sum_{i=1}^{m} q_{i}\left(\sqrt{\left[A_{*} u\right]_{i}\left[A_{*} v\right]_{i}}-\frac{1}{2}\left[A_{*} u\right]_{i}-\frac{1}{2}\left[A_{*} v\right]_{i}\right)\right\}$

Note: same as in the Election Polls case, $[\Psi(q)]_{\ell \ell^{\prime}}$ is efficiently computable and convex in $q$

$\Rightarrow$ Assuming the set $\mathcal{Q} \subset \mathbb{R}_{+}^{m}$ of allowed controls $q$ is convex, optimizing $\epsilon(q)$ over $q \in \mathcal{Q}$ is an explicit convex optimization problem. 


\section{Measurement Design problem in Gaussian o.s.}

$$
\omega=A[q] x+\xi, \xi \sim \mathcal{N}\left(0, I_{m}\right)
$$

[• $A[q]$ partially controlled sensing matrix; $\bullet q \in \mathcal{Q}$ : control parameters.]

is efficiently solvable if

$$
\begin{gathered}
A[q]=\operatorname{Diag}\left\{\sqrt{q_{1}}, \ldots, \sqrt{q_{m}}\right\} A_{*}, \\
\mathcal{Q} \subset \mathbb{R}_{+}^{m} \text { is a convex compact set }
\end{gathered}
$$

In this case, minimizing $\mathcal{Q}$-risk of test deciding up to closeness $\mathcal{C}$ on $L$ hypotheses

$$
H_{\ell}^{q}: \omega \sim \mathcal{N}\left(A[q] x, I_{m}\right), \quad x \in X_{\ell}, \quad 1 \leq \ell \leq L
$$

associated with nonempty convex compact sets $X_{\ell}$ reduces to solving convex problem

$$
\min _{q \in \mathcal{Q}} \Gamma(\Psi(q))
$$

where

$$
\Gamma(Q)=\left\|\left[\exp \left\{Q_{\ell \ell^{\prime}} / 8\right\} \chi_{\ell \ell^{\prime}}\right]_{\ell, \ell^{\prime} \leq L}\right\|_{2,2}
$$

is convex entrywise nondecreasing function of $L \times L$ matrix $Q$, and

$$
[\Psi(q)]_{\ell \ell^{\prime}}=\max _{u \in X_{\ell}, v \in X_{\ell^{\prime}}}\left[-\|A[q](u-v)\|_{2}^{2}\right]=-\min _{u \in X_{\ell}, v \in X_{\ell^{\prime}}}(u-v)^{T} A_{*}^{T} \operatorname{Diag}\{q\} A_{*}(u-v)
$$

is efficiently computable convex function of $q \in \mathcal{Q}$. 
Illustration: In some applications, "the physics" beyond Gaussian o.s. $\omega=$ $A x+\xi$ is as follows.

There are $m$ sensors measuring analogous vector-valued continuous time signal $x$ (nearly constant on the observation horizon) in the presence of noise. The output of sensor $\# i$ is

$$
\omega_{i}=\frac{1}{\left|\Delta_{i}\right|} \int_{\Delta_{i}}\left[a_{i, *}^{T} x+B_{i}(t)\right] d t
$$

- $\Delta_{i}$ : continuous time interval

- $B_{i}(t)$ : "Brownian motion:" $\frac{1}{|\Delta|} \int_{\Delta} B_{i}(t) d t \sim \mathcal{N}\left(0,|\Delta|^{-1}\right)$,

$\int_{\Delta} B_{i}(t) d t, \int_{\Delta^{\prime}} B_{i}(t) d t$ are independent when $\Delta \cap \Delta^{\prime}=\emptyset$

- Brownian motions $B_{i}(t)$ are independent across

- When all sensors work in parallel for unit time, we arrive at the standard Gaussian o.s. $\omega=A_{*} x+\xi, \xi \sim \mathcal{N}\left(0, I_{m}\right)$.

- When sensors work on consecutive segments $\Delta_{1}, \ldots, \Delta_{m}$ of durations $q_{i}=\left|\Delta_{i}\right|$, we arrive at

$$
\omega_{i}=a_{i, *}^{T} x+q_{i}^{-1 / 2} \xi_{i}, \quad \xi_{i} \sim \mathcal{N}(0,1) \text { are independent across } i
$$

Rescaling observations according to $\omega_{i} \mapsto \sqrt{q_{i}} \omega_{i}$, we arrive at the desired partially controlled observation scheme

$$
o \omega=\operatorname{Diag}\left\{\sqrt{q_{1}}, \ldots, \sqrt{q_{m}}\right\} A_{*} x+\xi, \xi \sim \mathcal{N}\left(0, I_{m}\right)
$$

A natural selection of $\mathcal{Q}$ is, e.g., $\mathcal{Q}=\left\{q \geq 0: \sum_{i} q_{i}=m\right\}$ (setting "time budget" to the same value as in the case of consecutive observations of duration 1 each). 


\section{HYPOTHESIS TESTING, III}

- Beyond simple observation schemes

- Simple families of distributions

- What is affine? Quadratic lifting 
Observation: A "common denominator" of minimum risk detectors for simple o.s.'s is their affinity in observations:

- the optimal detectors in Gaussian and Poisson o.s.'s are affine "as they are"

- encoding observation space $\Omega=\{1, \ldots, d\}$ of Discrete o.s. by vertices $e_{i}, i=1, \ldots, d$, of the standard simplex $\Delta_{d}=\left\{x \in \mathbb{R}^{d}: x \geq\right.$ $\left.0, \sum_{j} x_{j}=1\right\}$, every function on $\Omega$ becomes affine

$\Rightarrow$ we can treat optimal detector in Discrete o.s. as affine function on $\mathbb{R}^{d}$.

- operations with optimal detectors induced by taking direct products of basic simple o.s.'s or passing to repeated observations preserve affinity.

Claim: "Reasonable" (perhaps, sub-optimal) affine detectors can be found, in a computationally efficient way, in many important situations which are beyond simple o.s.'s. 


$$
\begin{gathered}
\mathcal{O}=\left((\Omega, \Pi),\left\{p_{\mu}(\cdot): \mu \in \mathcal{M}\right\}, \mathcal{F}\right) \\
\Phi_{\mathcal{O}}(\phi, \mu)=\operatorname{In}\left(\int \exp \{\phi(\omega)\} p_{\mu}(\omega) \Pi(d \omega)\right): \mathcal{F} \times \mathcal{M} \rightarrow \mathbb{R} \\
\text { convex in } \phi, \text { concave in } \mu
\end{gathered}
$$

In a simple o.s., our strategy to build a good detector for two hypotheses associated with convex compact sets $M_{1} \subset \mathcal{M}, M_{2} \subset \mathcal{M}$ was as follows:

In order to find minimum risk detector - to solve the optimization problem

$$
\min _{\phi \in \mathcal{F}} \max \left[\max _{\mu \in M_{1}} \int \exp \{-\phi(\omega)\} p_{\mu}(\omega) \Pi(d \omega), \max _{\nu \in M_{2}} \int \exp \{\phi(\omega)\} p_{\nu}(\omega) \Pi(d \omega)\right]
$$

reduced to

$$
\begin{gathered}
\min _{\phi \in \mathcal{F}} \frac{1}{2}\left[\max _{\mu \in M_{1}} \ln \left(\int \exp \{-\phi(\omega)\} p_{\mu}(\omega) \Pi(d \omega)\right)+\max _{\nu \in M_{2}} \ln \left(\int \exp \{\phi(\omega)\} p_{\nu}(\omega) \Pi(d \omega)\right)\right] \\
\Leftrightarrow \min _{\phi \in \mathcal{F}} \frac{1}{2}\left[\max _{\mu \in M_{1}} \Phi_{\mathcal{O}}(-\phi, \mu)+\max _{\nu \in M_{2}} \Phi_{\mathcal{O}}(\phi, \nu)\right]
\end{gathered}
$$

- We intend to use the same scheme, but replace " $\phi \in \mathcal{F}$ " with "affine $\phi$ ", and replace $\Phi_{\mathcal{O}}$ with convex in $\phi$ and concave in $\mu$ upper bound on

$$
\ln \left(\int \exp \{\phi(\omega)\} p_{\mu}(\omega) \Pi(d \omega)\right) \text {. }
$$




\section{Setup}

- Given an observation space $\Omega=\mathbb{R}^{d}$, consider a triple $\mathcal{H}, \mathcal{M}, \Phi$, where

- $\mathcal{H}$ is a nonempty closed convex set in $\Omega$ symmetric w.r.t. the origin,

- $\mathcal{M}$ is a compact convex set in some $\mathbb{R}^{n}$,

- $\Phi(h ; \mu): \mathcal{H} \times \mathcal{M} \rightarrow \mathbb{R}$ is a continuous function convex in $h \in \mathcal{H}$ and concave in $\mu \in \mathcal{M}$.

- $\mathcal{H}, \mathcal{M}$, and $\Phi$ specify a family $\mathcal{S}[\mathcal{H}, \mathcal{M}, \Phi]$ of probability distributions on $\Omega$. A probability distribution $P$ belongs to the family iff there exists $\mu \in \mathcal{M}$ such that

$$
\ln \left(\int_{\Omega} e^{h^{T} \omega} P(d \omega)\right) \leq \Phi(h ; \mu) \forall h \in \mathcal{H}
$$

We refer to $\mu$ ensuring $(*)$ as to parameter of distribution $P$.

Warning: A distribution $P$ may have many different parameters!

- We refer to triple $\mathcal{H}, \mathcal{M}, \Phi$ satisfying the above requirements as to regular data, and to $\mathcal{S}[\mathcal{H}, \mathcal{M}, \Phi]$ - as to the simple family of distributions induced by these data. 


\section{Example 1: Gaussian and sub-Gaussian distributions.}

When $\mathcal{M}=\{(u, \Theta)\} \subset \mathbb{R}^{d} \times$ int $\mathbf{S}_{+}^{d}$ is a convex compact set such that $\Theta \succ 0$ for all $(u, \Theta) \in \mathcal{M}, \mathcal{H}=\mathbb{R}^{d}$ and

$$
\Phi(h ; u, \Theta)=h^{T} y+\frac{1}{2} h^{T} \Theta h, \quad \mathcal{S}=\mathcal{S}[\mathcal{H}, \mathcal{M}, \Phi]
$$

contains all probability distributions $P$ which are sub-Gaussian with parameters $(u, \Theta)$, meaning that

$$
\ln \left(\int_{\Omega} e^{h^{T} \omega} P(d \omega)\right) \leq h^{T} u+\frac{1}{2} h^{T} \Theta h \forall h,
$$

and, in addition, the "parameter" $(u, \Theta)$ belongs to $\mathcal{M}$.

\section{Note:}

- $\mathcal{N}(u, \Theta) \in \mathcal{S}$ whenever $(u, \Theta) \in \mathcal{M}$; for $P=\mathcal{N}(u, \Theta),(1)$ is an identity

- Whenever $P$ is sub-Gaussian with parameters $(u, \Theta), u$ is the expectation of $P$ 


\section{Example 2: Poisson distributions.}

When $\mathcal{M} \subset \mathbb{R}_{+}^{d}$ is a convex compact set, $\mathcal{H}=\mathbb{R}^{d}$ and

$$
\Phi(h ; \mu)=\sum_{i=1}^{d} \mu_{i}\left(\mathrm{e}^{h_{i}}-1\right),
$$

$\mathcal{S}=\mathcal{S}[\mathcal{H}, \mathcal{M}, \Phi]$ contains distributions of all $d$-dimensional random vectors $\omega_{i}$ with independent across $i$ entries

$$
\omega_{i} \sim \operatorname{Poisson}\left(\mu_{i}\right)
$$

such that $\mu=\left[\mu_{1} ; \ldots ; \mu_{d}\right] \in \mathcal{M}$. 


\section{Example 3: Discrete distributions.}

When

$$
\mathcal{M}=\left\{\mu \in \mathbb{R}^{d}: \mu \geq 0, \sum_{j} \mu_{j}=1\right\}
$$

is the probabilistic simplex in $\mathbb{R}^{d}, \mathcal{H}=\mathbb{R}^{d}$ and

$$
\Phi(h ; \mu)=\ln \left(\sum_{i=1}^{d} \mu_{i} \mathrm{e}^{h_{i}}\right),
$$

$\mathcal{S}=\mathcal{S}[\mathcal{H}, \mathcal{M}, \Phi]$ contains all discrete distributions supported on the vertices of the probabilistic simplex. 


\section{Example 4: Distributions with bounded support.}

Let $X \subset \mathbb{R}^{d}$ be a nonempty convex compact set with support function $\phi_{X}(\cdot)$ :

$$
\phi_{x}(y)=\max _{x \in X} y^{T} x: \mathbb{R}^{d} \rightarrow \mathbb{R}^{d} .
$$

When $\mathcal{M}=X, \mathcal{H}=\mathbb{R}^{d}$ and

$$
\Phi(h ; \mu)=h^{T} \mu+\frac{1}{8}\left[\phi_{X}(h)+\phi_{X}(-h)\right]^{2},
$$

$\mathcal{S}=\mathcal{S}[\mathcal{H}, \mathcal{M}, \Phi]$ contains all probability distributions supported on $X$, and for such a distribution $P, \mu=\int_{X} \omega P(d \omega)$ is a parameter of $P$.

Note: When $G, 0 \in G$, is a convex compact set, the conclusion in Example 4 remains valid when function (2) is replaced with the smaller function

$$
\Phi(h ; \mu)=\min _{g \in G}\left[\mu^{T}(h-g)+\frac{1}{8}\left[\phi_{X}(h-g)+\phi_{X}(g-h)\right]^{2}+\phi_{X}(g)\right] .
$$




\section{"Calculus" of simple families of probability distributions}

- [summation] For $1 \leq \ell \leq L$, let $\lambda_{\ell}$ be reals, and let $\mathcal{H}_{\ell}, \mathcal{M}_{\ell}, \Phi_{\ell}$ be regular data with common observation space: $\mathcal{H}_{\ell} \subset \Omega=\mathbb{R}^{d}$. Setting

$$
\begin{aligned}
\mathcal{H} & =\left\{h \in \mathbb{R}^{d}: \lambda_{\ell} h \in \mathcal{H}_{\ell}, 1 \leq \ell \leq L\right\}, \\
\mathcal{M} & =\mathcal{M}_{1} \times \ldots \times \mathcal{M}_{L}, \\
\Phi\left(h ; \mu_{1}, \ldots, \mu_{L}\right) & =\sum_{\ell=1}^{L} \Phi_{\ell}\left(\lambda_{\ell} h ; \mu_{\ell}\right),
\end{aligned}
$$

we get regular data with the following property:

Whenever random vectors $\xi_{\ell} \sim P_{\ell} \in \mathcal{S}\left[\mathcal{H}_{\ell}, \mathcal{M}_{\ell}, \Phi_{\ell}\right], 1 \leq \ell \leq L$, are independent across $\ell$, the distribution $P$ of the random vector

$$
\xi=\sum_{\ell=1}^{L} \lambda_{\ell} \xi_{\ell}
$$

belongs to $\mathcal{S}[\mathcal{H}, \mathcal{M}, \Phi]$. Denoting by $\mu_{\ell}$ parameters of $P_{\ell}, \mu=$ $\left[\mu_{1} ; \ldots ; \mu_{L}\right]$ can be taken as parameter of $P$. 
- [direct product] For $1 \leq \ell \leq L$, let $\mathcal{H}_{\ell}, \mathcal{M}_{\ell}, \Phi_{\ell}$ be regular data with observation spaces $\Omega_{\ell}=\mathbb{R}^{d_{\ell}}$. Setting

$$
\begin{aligned}
\mathcal{H} & =\mathcal{H}_{1} \times \ldots \times \mathcal{H}_{L} \subset \Omega=\mathbb{R}^{d_{1}+\ldots+d_{L},} \\
\mathcal{M} & =\mathcal{M}_{1} \times \ldots \times \mathcal{M}_{L}, \\
\Phi\left(h_{1}, \ldots, h_{L} ; \mu_{1}, \ldots, \mu_{L}\right) & =\sum_{\ell=1}^{L} \Phi_{\ell}\left(h_{\ell} ; \mu_{\ell}\right),
\end{aligned}
$$

we get regular data with the following property:

Whenever $P_{\ell} \in \mathcal{S}\left[\mathcal{H}_{\ell}, \mathcal{M}_{\ell}, \Phi_{\ell}\right], 1 \leq \ell \leq L$, the direct product distribution $P=P_{1} \times \ldots \times P_{L}$ belongs to $\mathcal{S}[\mathcal{H}, \mathcal{M}, \Phi]$. Denoting by $\mu_{\ell}$ parameters of $P_{\ell}, \mu=\left[\mu_{1} ; \ldots ; \mu_{L}\right]$ can be taken as parameter of $P$. 
- [marginal] Let $\mathcal{H}, \mathcal{M}, \Phi$ be regular data with observation space $\mathbb{R}^{d}$, and let

$$
\omega \mapsto A \omega+a: \mathbb{R}^{d} \mapsto \Omega=\mathbb{R}^{\delta} .
$$

Setting

$$
\overline{\mathcal{H}}=\left\{h \in \mathbb{R}^{\delta}: A^{T} h \in \mathcal{H}\right\}, \bar{\Phi}(h ; \mu)=h^{T} a+\Phi\left(A^{T} h ; \mu\right),
$$

we get regular data $\overline{\mathcal{H}}, \mathcal{M}, \bar{\Phi}$ with the following property:

Whenever $\xi \sim P \in \mathcal{S}[\mathcal{H}, \mathcal{M}, \Phi]$, the distribution $\bar{P}$ of the random variable $\omega=A \xi+$ a belongs to the simple family $\mathcal{S}[\overline{\mathcal{H}}, \mathcal{M}, \bar{\Phi}]$, and parameter of $P$ is a parameter of $\bar{P}$ as well. 
Main observation: When deciding on simple families of distributions, affine tests and their risks can be efficiently computed via Convex Programming:

Theorem. Let $\mathcal{H}_{\chi}, \mathcal{M}_{\chi}, \Phi_{\chi}, \chi=1,2$, be two collections of regular data with compact $\mathcal{M}_{1}, \mathcal{M}_{2}$ and $\mathcal{H}_{1}=\mathcal{H}_{2}=: \mathcal{H}$, and let

$$
\Psi(h)=\max _{\mu_{1} \in \mathcal{M}_{1}, \mu_{2} \in \mathcal{M}_{2}} \underbrace{\frac{1}{2}\left[\Phi_{1}\left(-h ; \mu_{1}\right)+\Phi_{2}\left(h, \mu_{2}\right)\right]}_{\Phi\left(h ; \mu_{1}, \mu_{2}\right)}: \mathcal{H} \rightarrow \mathbb{R}
$$

Then $\Psi$ is efficiently computable continuous convex function, and for $h \in \mathcal{H}$, setting

$$
\phi_{h}(\omega)=h^{T} \omega+\underbrace{\frac{1}{2}\left[\max _{\mu_{1} \in \mathcal{M}_{1}} \Phi_{1}\left(-h ; \mu_{1}\right)-\max _{\mu_{2} \in \mathcal{M}_{2}} \Phi_{2}\left(h ; \mu_{2}\right)\right]}_{\varkappa},
$$

one has

$$
\operatorname{Risk}\left[\phi \mid \mathcal{P}_{1}, \mathcal{P}_{2}\right] \leq \exp \{\Psi(h)\}, \text { where } \mathcal{P}_{\chi}=\mathcal{S}\left[\mathcal{H}, \mathcal{M}_{\chi}, \Phi_{\chi}\right], \quad \chi=1,2 .
$$

In particular, if convex-concave function $\Phi\left(h ; \mu_{1}, \mu_{2}\right)$ possesses a saddle point $h_{*},\left(\mu_{1}^{*}, \mu_{2}^{*}\right)$ on $\mathcal{H} \times\left(\mathcal{M}_{1} \times \mathcal{M}_{2}\right)$, the affine detector

$$
\phi_{*}(\omega)=h_{*}^{T} \omega+\frac{1}{2}\left[\Phi_{1}\left(-h^{*} ; \mu_{1}^{*}\right)-\Phi_{2}\left(h^{*} ; \mu_{2}^{*}\right)\right]
$$

admits risk bound

$$
\operatorname{Risk}\left[\phi \mid \mathcal{P}_{1}, \mathcal{P}_{2}\right] \leq \exp \left\{\Phi\left(h^{*} ; \mu_{1}^{*}, \mu_{2}\right)\right\}
$$


Proof. Indeed, let $h \in \mathcal{H}$, and let

$$
\mu_{1}^{*} \in \underset{\mu_{1} \in \mathcal{M}_{1}}{\operatorname{Argmax}} \Phi_{1}\left(-h ; \mu_{1}\right), \mu_{2}^{*} \in \underset{\mu_{2} \in \mathcal{M}_{2}}{\operatorname{Argmax}} \Phi_{2}\left(h ; \mu_{2}\right) .
$$

When $P \in \mathcal{P}_{1}:=\mathcal{S}\left[\mathcal{H}, \mathcal{M}_{1}, \Phi_{1}\right]$, there is $\mu_{1} \in \mathcal{M}_{1}$ such that

$$
\mathbf{E}_{\omega \sim P}\left\{\mathrm{e}^{-h^{T} \omega} P(d \omega)\right\} \leq \mathrm{e}^{\Phi_{1}\left(-h ; \mu_{1}\right)},
$$

so that

$$
\mathbf{E}_{\omega \sim P}\left\{\mathrm{e}^{-\phi(\omega)} P(d \omega)\right\} \leq \mathrm{e}^{\Phi_{1}\left(-h ; \mu_{1}^{*}\right)-\kappa}=\mathrm{e}^{\psi(h)},
$$

implying that

$$
\operatorname{Risk}_{1}\left[\phi \mid \mathcal{P}_{1}, \mathcal{P}_{2}\right] \leq \mathrm{e}^{\psi(h)} .
$$

Similarly, when $\mu_{1} \in \mathcal{M}_{1}$, for some $\mu_{2} \in \mathcal{M}_{2}$ one has

$$
\begin{gathered}
\mathbf{E}_{\omega \sim P}\left\{\mathrm{e}^{h^{T} \omega} P(d \omega)\right\} \leq \mathrm{e}^{\Phi_{2}\left(h ; \mu_{2}\right)}, \\
\mathbf{E}_{\omega \sim P}\left\{\mathrm{e}^{\phi(\omega)} P(d \omega)\right\} \leq \mathrm{e}^{\Phi_{2}\left(h ; \mu_{2}^{*}\right)+\kappa}=\mathrm{e}^{\psi(h)},
\end{gathered}
$$

and

$$
\operatorname{Risk}_{2}\left[\phi \mid \mathcal{P}_{1}, \mathcal{P}_{2}\right] \leq \mathrm{e}^{\psi(h)} .
$$


Numerical illustration. Given convex compact subsets $X_{1}$ and $X_{2}$ of $X$, and observation

$$
\omega=A x+\sigma A \operatorname{Diag}\left\{\sqrt{x_{1}}, \ldots, \sqrt{x_{n}}\right\} \xi \quad\left[\xi \sim \mathcal{N}\left(0, I_{n}\right)\right]
$$

of an unknown signal $x$ known to belong to a given convex compact set $X \subset$ $\mathbb{R}_{++}^{n}$, we want to decide on the hypotheses

$$
H_{\chi}: x \in X_{\chi}, \quad \chi=1,2
$$

with risk $\leq 0.01$.

Novelty: Noise intensity depends on the signal!

- Introducing regular data $\mathcal{H}_{\chi}=\mathbb{R}^{n}, \mathcal{M}_{\chi}=X_{\chi}$,

$$
\Phi_{\chi}(h, \mu)=h^{T} A \mu+\frac{1}{2} \sigma^{2} h^{T}\left[A \operatorname{Diag}\{\mu\} A^{T}\right] h \quad[\chi=1,2]
$$

distribution of observations under $H_{\chi}$ belongs to $\mathcal{S}\left[\mathcal{H}, \mathcal{M}_{\chi}, \Phi_{\chi}\right]$.

- An affine detector for families $\mathcal{P}_{\chi}$ of distributions obeying $H_{\chi}, \chi=1,2$, is given by the saddle point of the function

$$
\Phi\left(h ; \mu_{1}, \mu_{2}\right):=\frac{1}{2}\left[h^{T}\left[\mu_{2}-\mu_{1}\right]+\frac{1}{2} \sigma^{2} h^{T} A \operatorname{Diag}\left\{\mu_{1}+\mu_{2}\right\} A^{T} h\right]
$$


Data: $n=16, \sigma=0.1$, target risk 0.01 ,

- $A=U$ Diag $\left\{0.01^{(i-1) / 15}, i \leq 16\right\} V^{T}$ with random orthogonal $U, V$,

- $X_{1}=\left\{x \in \mathbb{R}^{16:} \begin{array}{l}0.001 \leq x_{1} \leq \delta \\ 0.001 \leq x_{i} \leq 1, i \geq 2\end{array}\right\}$

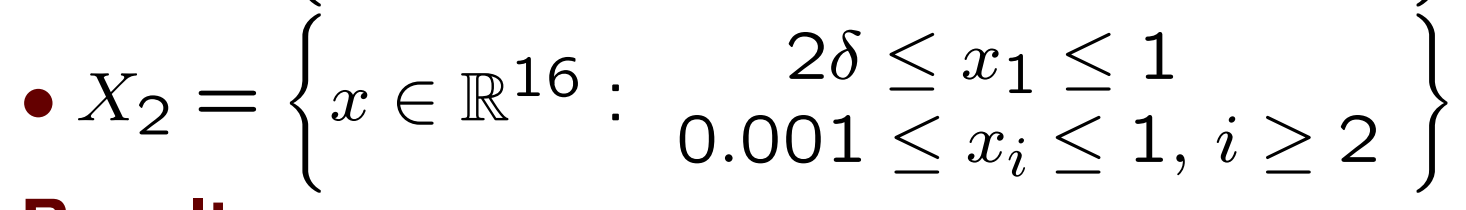

\section{Results:}

$$
\begin{aligned}
& \delta=0.1 \Rightarrow \operatorname{Risk}\left[\phi_{*} \mid \mathcal{P}_{1}, \mathcal{P}_{2}\right]=0.4346 \Rightarrow \text { 6-repeated observation } \\
& \delta=0.01 \Rightarrow \operatorname{Risk}\left[\phi_{*} \mid \mathcal{P}_{1}, \mathcal{P}_{2}\right]=0.9201 \Rightarrow 56 \text {-repeated observation }
\end{aligned}
$$

- Safe "Gaussian o.s. approximation" of the above observation scheme requires 37-repeated observations to handle $\delta=0.1$ and 3685 -repeated observation to handle $\delta=0.01$. 
Sub-Gaussian case. For $\chi=1,2$, let $U_{\chi} \subset \Omega=\mathbb{R}^{d}$ and $\mathcal{V}_{\chi} \subset$ int $\mathbf{S}_{+}^{d}$ be convex compact sets. Setting

$$
\mathcal{M}_{\chi}=U_{\chi} \times \mathcal{V}_{\chi}, \quad \Phi(h ; u, \Theta)=h^{T} u+\frac{1}{2} h^{T} \Theta h: \mathcal{H} \times \mathcal{M}_{\chi} \rightarrow \mathbb{R},
$$

the regular data $\mathcal{H}=\mathbb{R}^{d}, \mathcal{M}_{\chi}, \Phi$ specify the families $\mathcal{P}_{\chi}=\mathcal{S}\left[\mathbb{R}^{d}, U_{\chi} \times \mathcal{V}_{\chi}, \Phi\right]$ of sub-Gaussian distributions with parameters from $U_{\chi} \times \mathcal{V}_{\chi}$.

Saddle point problem responsible for design of affine detector for $\mathcal{P}_{1}, \mathcal{P}_{2}$ reads

$$
\text { SadVal }=\min _{h \in \mathbb{R}^{\mathbb{R}}} \max _{\substack{u_{1} \in v_{1}, u_{1} \in U_{2} \\ u_{1} \in v_{1}, \theta_{2} \in V_{2}}} \frac{1}{2}\left[h^{T}\left(u_{2}-u_{1}\right)+\frac{1}{2} h^{T}\left[\Theta_{1}+\Theta_{2}\right] h\right]
$$

- Saddle point $\left(h_{*} ;\left(u_{1}^{*}, u_{2}^{*}, \Theta_{1}^{*}, \Theta_{2}^{*}\right)\right)$ does exist and satisfies

$$
\begin{gathered}
h_{*}=\left[\Theta_{1}^{*}+\Theta_{2}^{*}\right]^{-1}\left[u_{1}^{*}-u_{2}^{*}\right], \\
\text { SadVal }=-\frac{1}{4}\left[u_{1}^{*}-u_{2}^{*}\right]\left[\Theta_{1}^{*}+\Theta_{2}^{*}\right]^{-1}\left[u_{1}^{*}-u_{2}^{*}\right]=-\frac{1}{4} h_{*}^{T}\left[u_{1}^{*}-u_{2}^{*}\right]
\end{gathered}
$$

- The associated affine detector and its risk are given by

$$
\begin{gathered}
\phi_{*}(\omega)=h_{*}^{T}\left[\omega-\frac{1}{2}\left[u_{1}^{*}+u_{2}^{*}\right]\right]=\left[u_{1}^{*}-u_{2}^{*}\right]^{T}\left[\Theta_{1}^{*}+\Theta_{2}^{*}\right]^{-1}\left[\omega-\frac{1}{2}\left[u_{1}^{*}+u_{2}^{*}\right]\right] \\
\operatorname{Risk}\left[\phi_{*} \mid \mathcal{P}_{1}, \mathcal{P}_{2}\right] \leq \exp \{\text { SadVal }\}=\exp \left\{-\frac{1}{4}\left[u_{1}^{*}-u_{2}^{*}\right]\left[\Theta_{1}^{*}+\Theta_{2}^{*}\right]^{-1}\left[u_{1}^{*}-u_{2}^{*}\right]\right\}
\end{gathered}
$$

Note: In the symmetric case $\mathcal{V}_{1}=\mathcal{V}_{2}\left(h_{*} ;\left(u_{1}^{*}, u_{2}^{*}, \Theta_{1}^{*}, \Theta_{2}^{*}\right)\right)$ can be selected to have $\Theta_{1}^{*}=\Theta_{2}^{*}=: \Theta_{*}$. In this case, the affine detector we end up with is the minimum risk detector for $\mathcal{P}_{1}, \mathcal{P}_{2}$. 


\section{What is "affine?" Quadratic Lifting}

We have developed a technique for building reasonable affine detectors for simple families of distributions.

Given observation $\zeta \sim P$, we can subject it to nonlinear transformation $\zeta \mapsto \omega=\psi(\zeta)$, e.g., to quadratic lifting

$$
\zeta \mapsto \omega=\left(\zeta, \zeta \zeta^{T}\right)
$$

and treat as our observation $\omega$ rather than $\zeta$. Affine in $\omega$ detectors are nonlinear in $\zeta$.

Example: Detectors affine in the quadratic lifting $\omega=\left(\zeta, \zeta \zeta^{T}\right)$ of $\zeta$ are exactly the quadratic functions of $\zeta$.

- We can try to apply our machinery for building affine detectors to nonlinear transformations of true observations, thus arriving at nonlinear detectors.

Bottleneck: To apply the outlined strategy to a pair $\mathcal{P}_{1}, \mathcal{P}_{2}$ of families of distributions of interest, we need to cover the families $\mathcal{P}_{1}^{+}, \mathcal{P}_{2}^{+}$of distributions of $\omega=\psi(\zeta)$ induced by distributions $P \in \mathcal{P}_{\chi}$ of $\zeta, \chi=1,2$, by simple families of distributions.

What is ahead: Simple "coverings" of quadratic lifts of (sub)Gaussian distributions. 
Situation: Given are:

- a compact nonempty set $U \subset \mathbb{R}^{n}$

- an affine mapping $u \mapsto \mathcal{A}(u)=A[u ; 1]: \mathbb{R}^{n} \rightarrow \mathbb{R}^{d}$

- a convex compact set $\mathcal{V} \subset \operatorname{int} \mathbf{S}_{+}^{d}$.

- The above data specify families of probability distributions of random observations

$$
\omega=\left(\zeta, \zeta \zeta^{T}\right), \zeta=\mathcal{A}(u)+\xi \in \mathbb{R}^{d},
$$

specifically,

- the family $\mathcal{G}[U, \mathcal{V}]$ of all distributions of $\omega$ induced by deterministic $u \in U$ and Gaussian noise $\xi \sim \mathcal{N}(0, \Theta \in \mathcal{V})$

— the family $\mathcal{S G}[U, \mathcal{V}]$ of all distributions of $\omega$ induced by deterministic $u \in U$ and sub-Gaussian, with parameters $(0, \Theta \in \mathcal{V})$ noise $\xi$

Goal: To cover $\mathcal{G}$ (or $\mathcal{S G}$ ) by a simple family of distributions. 


\section{Gaussian case}

Proposition. Given the above data $U, \mathcal{A}(u)=A[u ; 1], \mathcal{V}$, let us select

- $\gamma \in(0,1)$

- a computationally tractable convex compact set

$$
\mathcal{Z} \subset \mathcal{Z}^{+}=\left\{Z \in \mathbf{S}^{n+1}: Z \succeq 0, Z_{n+1, n+1}=1\right\}
$$

such that $[u ; 1][u ; 1]^{T} \in \mathcal{Z} \quad \forall u \in U$

- A matrix $\Theta_{*} \in \mathbf{S}^{d}$ and $\delta \in[0,2]$ such that

$$
\forall(\Theta \in \mathcal{V}): \Theta \preceq \Theta_{*} \text { and }\left\|\Theta^{1 / 2} \Theta_{*}^{-1 / 2}-I_{d}\right\|_{2,2} \leq \delta
$$

Let us set

$$
\begin{aligned}
& B=\left[\begin{array}{c}
A \\
0, \ldots, 0,1
\end{array}\right] \in \mathbb{R}^{(d+1) \times(n+1)}, \mathcal{M}=\mathcal{V} \times \mathcal{Z} \\
& \mathcal{H}=\left\{(h, H) \in \mathbb{R}^{d} \times \mathbf{S}^{d}:-\gamma \Theta_{*}^{-1} \preceq H \preceq \gamma \Theta_{*}^{-1}\right\} \\
& \Phi_{\mathcal{A}, \mathcal{Z}}(h, H ; \Theta, Z)=-\frac{1}{2} \ln \operatorname{Det}\left(I-\Theta_{*}^{1 / 2} H \Theta_{*}^{1 / 2}\right)+\frac{1}{2} \operatorname{Tr}\left(\left[\Theta-\Theta_{*}\right] H\right)+\frac{\delta(2+\delta)\left\|\Theta_{*}^{1 / 2} H \Theta_{*}^{1 / 2}\right\|_{F}^{2}}{2\left(1-\left\|\Theta_{*}^{1 / 2} H \Theta_{*}^{1 / 2}\right\|\right)} \\
& +\frac{1}{2} \operatorname{Tr}\left(Z B^{T}\left[\left[\begin{array}{c|c}
H & h \\
\hline h^{T} &
\end{array}\right]+[H, h]^{T}\left[\Theta_{*}^{-1}-H\right]^{-1}[H, h]\right] B\right): \mathcal{H} \times \mathcal{M} \rightarrow \mathbb{R}
\end{aligned}
$$

Then $\mathcal{H}, \mathcal{M}, \Phi_{\mathcal{A}, \mathcal{Z}}$ is efficiently computable regular data, and

$$
\mathcal{G}[U, \mathcal{V}] \subset \mathcal{S}\left[\mathcal{H}, \mathcal{M}, \Phi_{\mathcal{A}, \mathcal{Z}}\right]
$$




\section{Sub-Gaussian case}

Proposition. Given the above data $U, \mathcal{A}(u)=A[u ; 1], \mathcal{V}$, let us select

- $\gamma, \gamma^{+} \in(0,1)$ with $\gamma<\gamma^{+}$

- a computationally tractable convex compact set

$$
\mathcal{Z} \subset \mathcal{Z}^{+}=\left\{Z \in \mathbf{S}^{n+1}: Z \succeq 0, Z_{n+1, n+1}=1\right\}
$$

such that $[u ; 1][u ; 1]^{T} \in \mathcal{Z} \quad \forall u \in U$

- A matrix $\Theta_{*} \in \mathbf{S}^{d}$ and $\delta \in[0,2]$ such that

$$
\forall(\Theta \in \mathcal{V}): \Theta \preceq \Theta_{*} \text { and }\left\|\Theta^{1 / 2} \Theta_{*}^{-1 / 2}-I_{d}\right\|_{2,2} \leq \delta
$$

Let us set

$$
\begin{aligned}
& B=\left[\begin{array}{c}
A \\
0, \ldots, 0,1
\end{array}\right] \in \mathbb{R}^{(d+1) \times(n+1)}, \\
& \mathcal{H}=\left\{(h, H) \in \mathbb{R}^{d} \times \mathbf{S}^{d}:-\gamma \Theta_{*}^{-1} \preceq H \preceq \gamma \Theta_{*}^{-1}\right\}, \mathcal{M}=\mathcal{Z} \\
& \mathcal{H}^{+}=\left\{(h, H, G) \in \mathbb{R}^{d} \times \mathbf{S}^{d} \times \mathbf{S}^{d}:-\gamma^{+} \Theta_{*}^{-1} \preceq H \preceq G \preceq \gamma^{+} \Theta_{*}^{-1}, 0 \preceq G\right\} \\
& \Phi_{\mathcal{A}, \mathcal{Z}}(h, H ; Z)=\min _{G:(h, H, G) \in \mathcal{H}^{+}}\left\{-\frac{1}{2} \ln \operatorname{Det}\left(I-\Theta_{*}^{1 / 2} G \Theta_{*}^{1 / 2}\right)\right. \\
& \left.+\frac{1}{2} \operatorname{Tr}\left(Z B^{T}\left[\left[\begin{array}{l|l}
H & h \\
\hline h^{T} &
\end{array}\right]+[H, h]^{T}\left[\Theta_{*}^{-1}-G\right]^{-1}[H, h]\right] B\right)\right\}: \mathcal{H} \times \mathcal{M} \rightarrow \mathbb{R}
\end{aligned}
$$

Then $\mathcal{H}, \mathcal{M}, \Phi_{\mathcal{A}, \mathcal{Z}}$ is efficiently computable regular data, and

$$
\mathcal{S G}[U, \mathcal{V}] \subset \mathcal{S}\left[\mathcal{H}, \mathcal{M}, \Phi_{\mathcal{A}, \mathcal{Z}}\right]
$$


How to specify $\mathcal{Z}$ ? To apply the above construction, one should specify a computationally tractable convex compact set

$$
\mathcal{Z} \subset \mathcal{Z}^{+}=\left\{Z \in \mathrm{S}^{n+1}: Z \succeq 0, Z_{n+1, n+1}=1\right\}
$$

the smaller the better, such that $u \in U \rightarrow[u ; 1][u ; 1]^{T} \in \mathcal{Z}$

- The ideal selection is

$$
\mathcal{Z}=\mathcal{Z}[U]=\operatorname{Conv}\left\{[u ; 1][u ; 1]^{T}: u \in U\right\}
$$

However: $\mathcal{Z}[U]$ usually is computationally intractable.

Important exception:

$$
Q \succ 0, U=\left\{u: u^{T} Q u \leq 1\right\} \Rightarrow \mathcal{Z}[U]=\left\{Z \in \mathcal{Z}^{+}: \operatorname{Tr}(Z Q) \leq 1\right\}
$$


"Simple" case: When $U$ is given by $S$ quadratic inequalities:

$$
U=\left\{u \in \mathbb{R}^{n}:[u ; 1]^{T} Q_{s}[u ; 1] \leq q_{s}, 1 \leq s \leq S\right\}
$$

we can set

$$
\mathcal{Z}=\left\{Z \in \mathbf{S}^{n+1}: Z \succeq 0, Z_{n+1, n+1}=1, \operatorname{Tr}\left(Q_{s} Z\right) \leq q_{s}, 1 \leq s \leq S\right\} .
$$

Warning: $(*)$ can yield very conservative outer approximation of $\mathcal{Z}[U]$. This conservatism with luck can be reduced by passing from the original description of $U$ to an equivalent one, with emphasis on eliminating/updating linear constraints. For example,

- a constraint of the form $\left|a^{T} x-c\right| \leq r$ should be replaced with

$$
\left(a^{T} x-c\right)^{2} \leq r^{2}
$$

Note: every linear constraint in the description of $U$ can be written as $\alpha-a^{T} x \geq 0$ and augmented by redundant constraint $a^{T} x \geq \beta$, with appropriately selected $\beta$. The resulting pair of constraints is equivalent to $\left|a^{T} x-c\right| \leq r$ with $c=\frac{1}{2}[\alpha+\beta]$ and $r=\frac{1}{2}[\alpha-\beta]$.

- It could make sense to write the linear constraints in the description of $U$ in the form $\alpha-a^{T} x \geq 0$ and add to these constraints their pairwise products. 


\section{Quadratic Lifting - Does it Pay?}

Situation: Assume that we are given

- convex compact sets $U_{\chi} \subset \mathbb{R}^{n} \chi, \chi=1,2$

- affine mappings $u_{\chi} \mapsto \mathcal{A}_{\chi}\left(u_{\chi}\right): \mathbb{R}^{n_{\chi}} \rightarrow \mathbb{R}^{d}, \chi=1,2$

- convex compact sets $\mathcal{V}_{\chi} \subset \operatorname{int} \mathbf{S}_{+}^{d}, \chi=1,2$.

These data define families $\mathcal{G}_{\chi}$ of Gaussian distributions:

$$
\mathcal{G}_{\chi}=\left\{\mathcal{N}\left(\mathcal{A}_{\chi}\left(u_{\chi}\right), \Theta_{\chi}\right): u_{\chi} \in U_{\chi}, \Theta_{\chi} \in \mathcal{V}_{\chi}\right\}
$$


We can build two types of detectors for $\mathcal{G}_{1}, \mathcal{G}_{2}$ :

- Affine detector $\phi_{\text {aff }}$ yielded by the solution to the saddle point problem

$$
\begin{aligned}
& \text { SadVal aff }=\min _{h \in \mathbb{R}^{d}} \max _{\substack{u_{1} \in U_{1}, u_{2} \in U_{2} \\
\Theta_{1} \in \mathcal{V}_{1}, \Theta_{2} \in \mathcal{V}_{2}}} \frac{1}{2}\left[h^{T}\left[\mathcal{A}_{2}\left(u_{2}\right)-\mathcal{A}_{1}\left(u_{1}\right)\right]+\frac{1}{2} h^{T}\left[\Theta_{1}+\Theta_{2}\right] h\right], \\
& \operatorname{Risk}\left[\phi_{\text {aff }} \mid \mathcal{G}_{1}, \mathcal{G}_{2}\right] \leq \exp \left\{\left.\operatorname{SadVal}\right|_{\text {aff }}\right\}
\end{aligned}
$$

- Quadratic detector $\phi_{\text {lift }}$ yielded by the solution to the saddle point problem

$$
\begin{aligned}
& \text { SadVal lift }=\min _{(h, H) \in \mathcal{H}} \max _{\substack{\Theta_{1} \in \mathcal{V}_{1} \\
\Theta_{2} \in \mathcal{V}_{2}}} \frac{1}{2}\left[\Phi_{\mathcal{A}_{1}, \mathcal{Z}_{1}}\left(-h,-H ; \Theta_{1}\right)+\Phi_{\mathcal{A}_{2}, \mathcal{Z}_{2}}\left(h, H ; \Theta_{2}\right)\right] \text {, } \\
& \operatorname{Risk}\left[\phi_{\text {lift }} \mid \mathcal{G}_{1}, \mathcal{G}_{2}\right] \leq \exp \left\{\left.\operatorname{SadVa|}\right|_{\text {lift }}\right\}
\end{aligned}
$$

Observation: Assume that sets $\mathcal{V}_{\chi}$ contain $\succeq$-largest elements. Then with proper selection of the "design parameters" $\mathcal{Z}_{\chi}, \Theta_{*}^{(\chi)}$ participating in the construction of $\Phi_{\mathcal{A}_{\chi}, \mathcal{Z}_{\chi}}, \chi=1,2$, passing from affine to quadratic detectors helps:

$$
\text { SadVal }_{\text {lift }} \leq \text { SadVal }_{\text {aff }}
$$




\section{Numerical illustration:}

- $U_{1}=U_{1}^{\rho}=\left\{u \in \mathbb{R}^{12}: u_{i} \geq \rho, 1 \leq i \leq 12\right\}$,

- $U_{2}=U_{2}^{\rho}=-U_{1}^{\rho}$

- $A_{1}=A_{2} \in \mathbb{R}^{8 \times 13}$

- $\mathcal{V}_{\chi}=\left\{\Theta_{*}^{(\chi)}=\sigma_{\chi}^{2} I_{8}\right\}$

\begin{tabular}{||c||c|c||c|c|c||}
\hline$\rho$ & $\sigma_{1}$ & $\sigma_{2}$ & $\begin{array}{c}\text { unrestricted } \\
H \text { and } h\end{array}$ & $H=0$ & $h=0$ \\
\hline 0.5 & 2 & 2 & 0.31 & 0.31 & 1.00 \\
\hline 0.5 & 1 & 4 & 0.24 & 0.39 & 0.62 \\
\hline 0.01 & 1 & 4 & 0.41 & 1.00 & 0.41 \\
\hline
\end{tabular}

Risk of quadratic detector $\phi(\zeta)=h^{T} \zeta+\frac{1}{2} \zeta^{T} H \zeta+\varkappa$

- when deciding on families of Gaussian distributions with common covariance matrix and expectations varying in associated with the families convex sets, passing from affine to quadratic detectors does not help

- in general, both affine and purely quadratic components in a quadratic detector are useful.

- when deciding on families of Gaussian distributions in the case where distributions from different families can have close expectations, quadratic detectors are useful, while affine ones are not. 


\section{Illustration: Simple Change Point Detection}

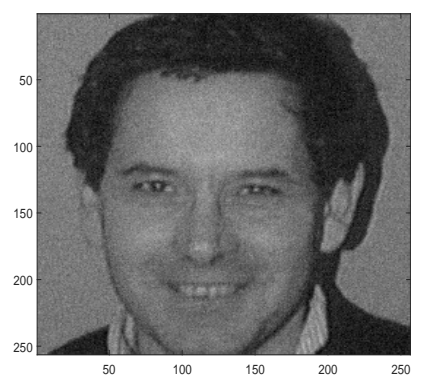

\# 1

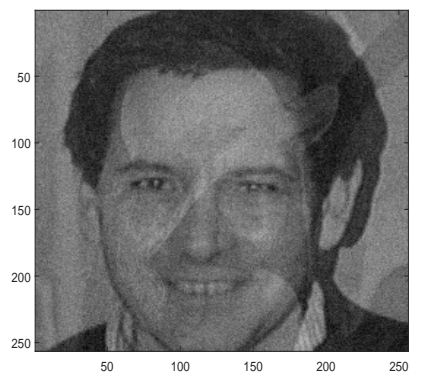

\# 7

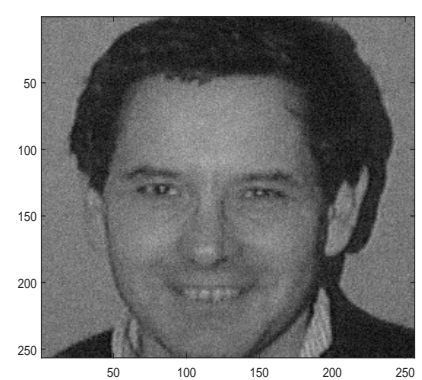

\# 2

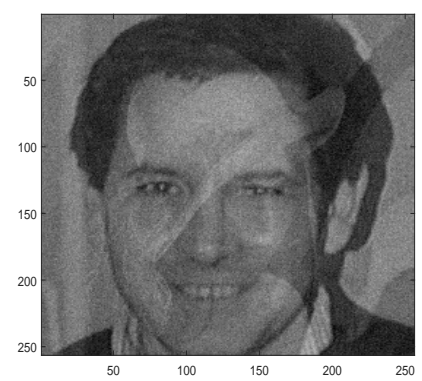

\# 8

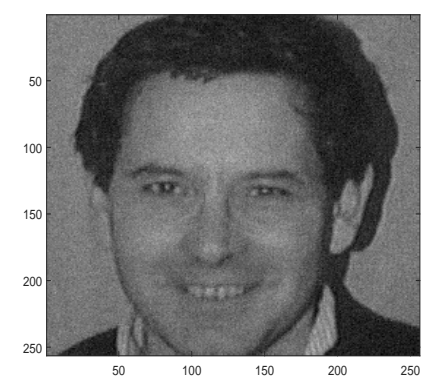

\# 3

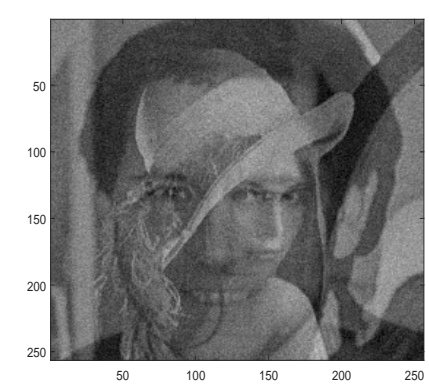

\# 15

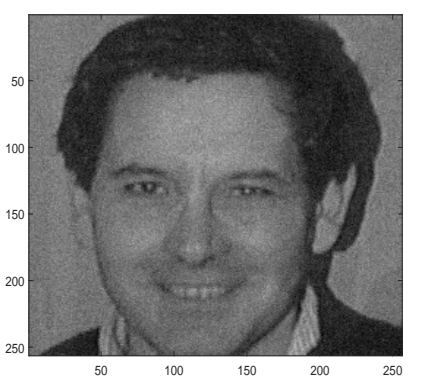

\# 4

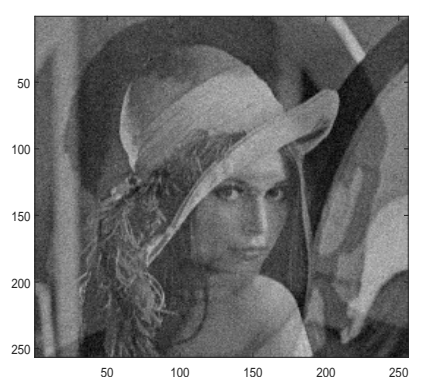

\# 20

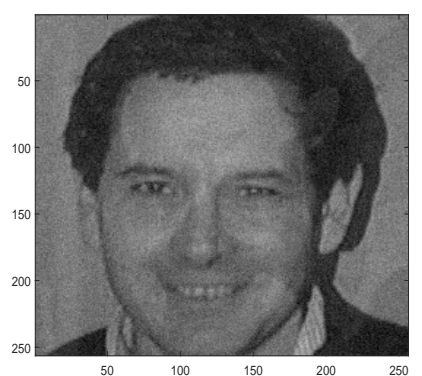

\# 5

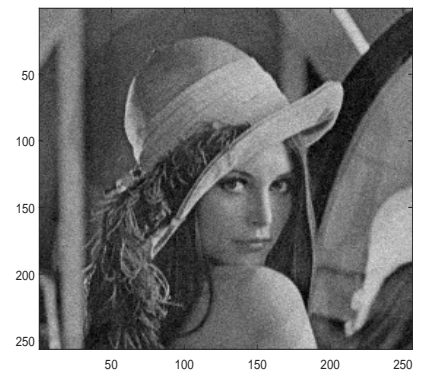

\# 28

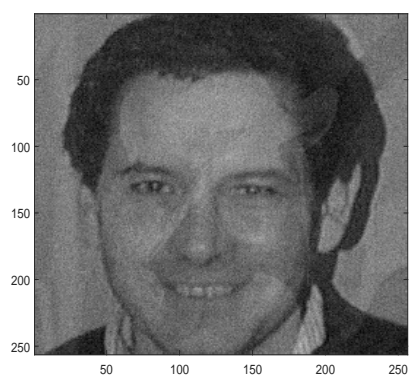

\# 6

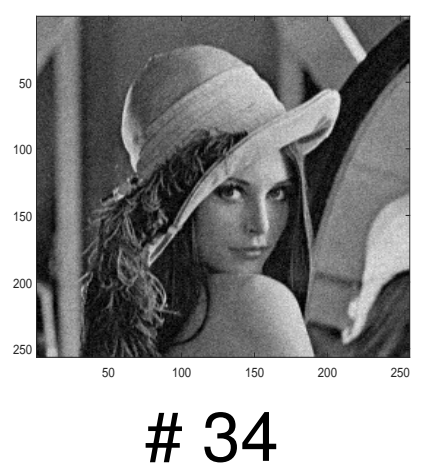

Frames from a noisy "movie"

When the picture starts to change? 
Model: We observe one by one "vectors" (2D images)

$$
\omega_{t}=x_{t}+\xi_{t}
$$

- $x_{t}$ : deterministic image

- $\xi_{t} \sim \mathcal{N}\left(0, \sigma^{2} I_{d}\right)$ : independent across observation noises.

Note: We know the range $[\underline{\sigma}, \bar{\sigma}]$ of $\sigma$, but perhaps do not know $\sigma$ exactly.

- We know that $x_{1}=x_{2}$ and want to check whether $x_{1}=\ldots=x_{K}$ ("no change") or there is a change.

Goal: Given an upper bound $\epsilon>0$ on the probability of false alarm, we want to design a sequential change detection routine capable to detect change, if any. 


\section{Approach:}

- Pass from observations $\omega_{t}, 1 \leq t \leq K$, to observations

$$
\zeta_{t}=\omega_{t}-\omega_{1}=\underbrace{x_{t}-x_{1}}_{y_{t}}+\underbrace{\xi_{t}-\xi_{1}}_{\eta_{t}}, \quad 2 \leq t \leq K
$$

- Test hypothesis $H_{0}: y_{2}=\ldots=y_{K}=0$ vs. alternative

$$
\bigcup_{k=2}^{K} H_{k}^{\rho}, H_{k}^{\rho}: y_{2}=\ldots=y_{k-1}=0,\left\|y_{k}\right\|_{2} \geq \rho
$$

via our machinery for testing

$$
\text { red hypothesis } H_{0}
$$

VS.

$$
\text { brown hypotheses } H_{2}^{\rho}, \ldots, H_{K}^{\rho}
$$

via quadratic liftings $\zeta_{t} \zeta_{t}^{T}$ of observations $\zeta_{t}$ up to closeness

$\mathcal{C}$ : all brown hypotheses are close to each other and are not close to the red hypothesis

- We intend to find the smallest $\rho$ for which the $\mathcal{C}$-risk of the resulting inference is $\leq \epsilon$, and utilize this inference in change point detection. 


\section{How It Works}

Setup: $\operatorname{dim} y=256^{2}=65536, \bar{\sigma}=10, \bar{\sigma}^{2} / \underline{\sigma}^{2}=2, K=9, \epsilon=0.01$

Detector: At time $t=2, \ldots, K$, compute

$$
\phi_{*}\left(\zeta_{t}\right)=-2.7138 \frac{\left\|\zeta_{t}\right\|_{2}^{2}}{10^{5}}+366.9548 .
$$

$\phi_{*}\left(\zeta_{t}\right)<0 \Rightarrow$ conclude that the change took place and terminate

$\phi_{*}\left(\zeta_{t}\right) \geq 0 \Rightarrow$ conclude that there was no change so far and proceed to the next image, if any

- When red hypothesis $H_{0}$ holds true, the probability not to claim change on time horizon $2, \ldots, K$ is at least 0.99 .

- When a brown hypothesis $H_{k}^{\rho}$ holds true, the change at time $\leq K$ is detected with probability at least 0.99 , provided $\rho \geq \rho_{*}=2716.6$ (average per pixel energy in $y_{k}$ at least by $12 \%$ larger than $\bar{\sigma}^{2}$ )

- No test can 0.99-reliably decide via $\zeta_{1}, \ldots, \zeta_{k}$ on $H_{k}^{\rho}$ vs. $H_{0}$, provided $\rho / \rho_{*}<$ 0.965.

- In the movie, the change which takes place at $t=3$ is detected at $t=4$. 


\section{ESTIMATING SIGNALS IN GAUSSIAN O.S. AND BEYOND}

- Problem of interest

- Developing tools, Optimization

- Conic Programming

- Conic Duality

- Developing tools, Statistics

- Gauss-Markov Theorem

- Optimizing linear estimates

- Near-optimality of linear estimates

- Byproduct on Semidefinite Relaxation 
Situation: "In the nature" there exists a signal $x$ known to belong to a given convex compact set $\mathcal{X} \subset \mathbb{R}^{n}$. We observe corrupted by noise affine image of the signal:

$$
\omega=A x+\sigma \xi \in \Omega=\mathbb{R}^{m}
$$

- $A$ : given $m \times n$ sensing matrix

- $\xi$ : random observation noise

Goal: To recover the image $B x$ of $x$ under a given linear mapping

- $B$ : given $\nu \times n$ matrix.

Risk of a candidate estimate $\widehat{x}(\cdot): \Omega \rightarrow \mathbb{R}^{\nu}$ is defined as

$$
\operatorname{Risk}[\widehat{x} \mid \mathcal{X}]=\sup _{x \in \mathcal{X}} \sqrt{\mathbf{E}_{\xi}\left\{\|B x-\widehat{x}(A x+\sigma \xi)\|_{2}^{2}\right\}}
$$

Risk $^{2}$ is the worst-case, over $x \in \mathcal{X}$, expected $\|\cdot\|_{2}^{2}$ recovery error.

Ideal goal: To build the minmax optimal estimate $\widehat{x}_{*}$ achieving the minmax risk

$$
\operatorname{RiskOpt}[\mathcal{X}]=\inf _{\widehat{x}(\cdot)} \operatorname{Risk}[\widehat{x} \mid \mathcal{X}]
$$


Agenda: Under appropriate assumptions on $\mathcal{X}$, we show that

- One can build, in a computationally efficient fashion, (nearly) the best, in terms of risk, estimate in the family of linear estimates

$$
\widehat{x}(\omega)=\widehat{x}_{H}(\omega)=H^{T} \omega \quad\left[H \in \mathbb{R}^{m \times \nu}\right]
$$

- The resulting linear estimate is nearly optimal among all estimates, linear and nonlinear alike. 


\section{Why linear estimates?}

As it was announced, a "nearly optimal" linear estimate can be built in a computationally efficient fashion.

In contrast, Exactly minimax optimal estimate is unknown even in the simplest case when the observation is

$$
\omega=x+\eta
$$

with $\eta \sim \mathcal{N}\left(0, \sigma^{2}\right)$ and $x \in \mathcal{X}=[-1,1]$

Note: The statistical "magic wand" - Maximum Likelihood Estimate - can be heavily nonoptimal already in the Simple case. 


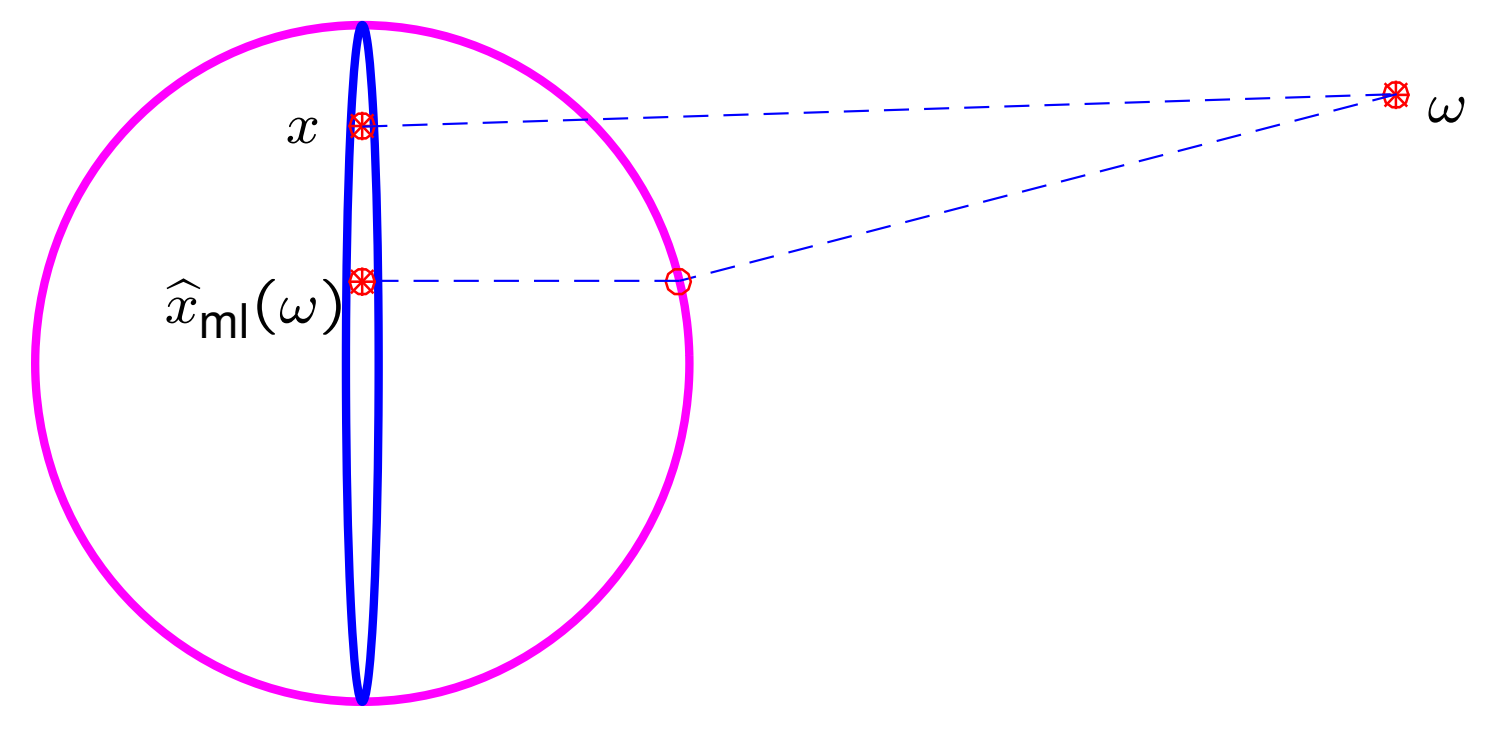

blue: $\mathcal{X}$ magenta: $A \mathcal{X}$

- $\mathcal{X}=\left\{x \in \mathbb{R}^{n}: x_{n}^{2}+\epsilon^{-2} \sum_{i=1}^{n-1} x_{i}^{2} \leq 1\right\}$

- $A=\operatorname{Diag}\{1 / \epsilon, \ldots, 1 / \epsilon, 1\}, \quad \eta \sim \mathcal{N}\left(0, \sigma^{2} I_{n}\right), \quad B=I_{n}$

$\Rightarrow$ MLE: $\widehat{x}_{\mathrm{ml}}(\omega)=A^{-1} \cdot \operatorname{argmin}_{\|u\|_{2} \leq 1}\|\omega-u\|_{2}$

When $\sigma \ll 1, \sigma^{2} n \geq O(1)$, and $\epsilon \leq O(\sigma)$, the risk of MLE is $O(1)$, while the risk of the linear estimate $\widehat{x}(\omega)=\omega_{n}$ is $O(\sigma) \ll O(1)$. 


\section{$\omega=A x+\sigma \xi \& x \in \mathcal{X}^{\prime} \Rightarrow \widehat{x}_{H}(\omega):=H^{T} \omega \approx B x$}

\section{Design of linear estimates: challenges}

Fact: Unless $A$ and $B$ are simple - just diagonal, - analytical risk analysis/design of linear estimates is problematic even in the Simple case where $\xi \sim \mathcal{N}(0, I)$ and $\mathcal{X}=\left\{x \in \mathbb{R}^{n}: \sum_{i} a_{i}^{2} x_{i}^{2} \leq 1\right\}$ is an ellipsoid.

Reason: Optimal minimax risk is determined by difficult to represent and analyze interplay between "geometries" of $A, B, \mathcal{X}$ :

- on one hand, poor conditioning of $A$ can make impossible good recovery of some components of $x$ even in a low noise

- on the other hand, the geometries of $\mathcal{X}$ and/or $B$ can make "difficult to recover" components of $x$ irrelevant - these components can be a priori small due to the geometry of the signal set $\mathcal{X}$, or can be suppressed by $B$ due to the geometry of $B$.

Surprisingly, difficulties basically disappear when passing from descriptive analytical to computationally efficient operational design and risk analysis of estimates. Specifically, in Simple case with no assumptions on $A, B$ (and even far beyond) one can build in a computationally efficient fashion provably minimax optimal, up to logarithmic factors, linear estimates. 
Developing tools, Optimization: "structure-revealing" representation of Convex Problems: Conic Programming

- When passing from a Linear Programming program

$$
\min _{x}\left\{c^{T} x: A x-b \geq 0\right\}
$$

to a nonlinear convex one, the traditional wisdom is to replace linear inequality constraints

with nonlinear ones:

$$
a_{i}^{T} x-b_{i} \geq 0
$$

$$
g_{i}(x) \geq 0 \quad\left[g_{i} \text { are concave }\right]
$$

- There exists, however, another way to introduce nonlinearity, namely, to replace the coordinate-wise vector inequality

$$
y \geq z \Leftrightarrow y-z \in \mathbb{R}_{+}^{m}=\left\{u \in \mathbb{R}^{m}: u_{i} \geq 0 \forall i\right\} \quad\left[y, z \in \mathbb{R}^{m}\right]
$$

with another vector inequality

$$
y \geq_{\mathbf{K}} z \Leftrightarrow y-z \in \mathbf{K} \quad\left[y, z \in \mathbb{R}^{m}\right]
$$

where $\mathbf{K}$ is a regular cone (i.e., closed, pointed and convex cone with a nonempty interior) in $\mathbb{R}^{m}$. 


$$
y \geq_{\mathbf{K}} z \Leftrightarrow y-z \in \mathbf{K} \quad\left[y, z \in \mathbb{R}^{m}\right]
$$

$\mathbf{K}$ : closed, pointed and convex cone in $\mathbb{R}^{m}$ with a nonempty interior.

Requirements on $\mathbf{K}$ ensure that $\geq_{\mathbf{K}}$ obeys the usual rules for inequalities:

- $\geq_{\mathbf{K}}$ is a partial order:

$$
\begin{aligned}
& x \geq_{\mathbf{K}} x \quad \forall x \\
& \left(x \geq_{\mathbf{K}} y \quad \& \quad y \geq_{\mathbf{K}} x\right) \Rightarrow x=y \\
& \left(x \geq_{\mathbf{K}} y, \quad y \geq_{\mathbf{K}} z\right) \Rightarrow x \geq_{\mathbf{K}} z
\end{aligned}
$$

[reflexivity] [antisymmetry]

[transitivity]

- $\geq_{K}$ is compatible with linear operations: the validity of $\geq_{K}$ inequality is preserved when we multiply both sides by the same nonnegative real and add to it another valid $\geq_{\mathrm{K}}$-inequality;

- in a sequence of $\geq_{K^{-}}$-inequalities, one can pass to limits:

$$
\left\{a_{i} \geq_{\mathbf{K}} b_{i}, i=1,2, \ldots \& a_{i} \rightarrow a \& b_{i} \rightarrow b\right\} \Rightarrow a \geq_{\mathbf{K}} b
$$




$$
y \geq_{\mathbf{K}} z \Leftrightarrow y-z \in \mathbf{K} \quad\left[y, z \in \mathbb{R}^{m}\right]
$$

$\mathrm{K}$ : closed, pointed and convex cone in $\mathbb{R}^{m}$ with a nonempty interior.

- one can define the strict version $>_{\mathrm{K}}$ of $\geq_{\mathrm{K}}$ :

$$
a>_{\mathbf{K}} b \Leftrightarrow a-b \in \operatorname{int} \mathbf{K} .
$$

Arithmetics of $>_{\mathrm{K}}$ and $\geq_{\mathrm{K}}$ inequalities is completely similar to the arithmetics of the usual coordinate-wise $>$ and $\geq$. 


\section{LP problem:}

$$
\min _{x}\left\{c^{T} x: A x-b \geq 0\right\} \Leftrightarrow \min _{x}\left\{c^{T} x: A x-b \in \mathbb{R}_{+}^{m}\right\}
$$

\section{General Conic problem:}

$$
\min _{x}\left\{c^{T} x: A x-b \geq_{\mathbf{K}} 0\right\} \Leftrightarrow \min _{x}\left\{c^{T} x: A x-b \in \mathbf{K}\right\}
$$

- $(A, b)$ - data of conic problem

- $\mathbf{K}$ - structure of conic problem

\section{Remarks:}

- Every convex problem admits equivalent conic reformulation

- With conic formulation, convexity is "built in"; with the standard MP formulation convexity should be kept in mind as an additional property.

(??) A general convex cone has no more structure than a general convex function. Why conic reformulation is "structure-revealing"?

(!!) Just 3 types of cones allow to represent an extremely wide spectrum ("essentially all") of convex problems! 


$$
\min _{x}\left\{c^{T} x: A x-b \geq_{\mathbf{K}} 0\right\} \Leftrightarrow \min _{x}\left\{c^{T} x: A x-b \in \mathbf{K}\right\}
$$

Three Magic Families of cones:

- $\mathcal{L P}$ : Nonnegative orthants $\mathbb{R}_{+}^{m}$ - direct products of $m$ nonnegative rays

$$
\mathbb{R}_{+}=\{s \in \mathbb{R}: s \geq 0\}
$$

giving rise to Linear Programming programs

$$
\min _{s}\left\{c^{T} x: a_{\ell}^{T} x-b_{\ell} \geq 0,1 \leq \ell \leq q\right\} .
$$

- $\mathcal{C Q P}$ : Direct products of Lorentz cones

$$
\mathbf{L}_{+}^{p}=\left\{u \in \mathbb{R}^{p}: u_{p} \geq\left(\sum_{i=1}^{p-1} u_{i}^{2}\right)^{1 / 2}\right\}
$$

giving rise to Conic Quadratic programs

$$
\min _{x}\left\{c^{T} x:\left\|A_{\ell} x-b_{\ell}\right\|_{2} \leq c_{\ell}^{T} x-d_{\ell}, 1 \leq \ell \leq q\right\} .
$$


- $S \mathcal{S P}$ : Direct products of Semidefinite cones

$$
\mathbf{S}_{+}^{p}=\left\{M \in \mathbf{S}^{p}: M \succeq 0\right\}
$$

giving rise to Semidefinite programs

$$
\min _{x}\{c^{T} x: \underbrace{\lambda_{\min }\left(\mathcal{A}^{\ell}(x)\right) \geq 0}_{\Leftrightarrow \mathcal{A}^{\ell}(x) \succeq 0}, 1 \leq \ell \leq q\} .
$$

where $\mathbf{S}^{p}$ is the space of $p \times p$ real symmetric matrices, $\mathcal{A}_{\ell}(x) \in \mathbf{S}^{p}$ are affine in $x$ and $\lambda_{\min }(S)$ is the minimal eigenvalue of $S \in \mathrm{S}^{p}$.

Note: Constraint $\mathcal{A}(x) \succeq 0$ stating that a symmetric matrix affinely depending on decision variables is $\succeq 0$ is called LMI - Linear Matrix Inequality.

For details, see https://www2.isye.gatech.edu/ nnemirovs/lmco_run.pdf 


\section{What can be reduced to $\mathcal{L P} / \mathcal{C} \mathcal{Q P} / \mathcal{S D P}$ ? Calculus of Conic Programs}

Let $\mathcal{K}$ be a family of regular cones closed w.r.t. taking direct products.

Definition: $A \mathcal{K}$-representation of a set $X \subset \mathbb{R}^{n}$ is a representation

$$
X=\left\{x \in \mathbb{R}^{n}: \exists u \in \mathbb{R}^{m}: A x+B u-b \in \mathbf{K}\right\}
$$

where $\mathrm{K} \in \mathcal{K} . X$ is called $\mathcal{K}$-representable, if $X$ admits a $\mathcal{K}-r$.

Note: Minimizing a linear objective $c^{T} x$ over a $\mathcal{K}$-representable set $X$ reduces to a conic program on a cone from $\mathcal{K}$. Indeed, given $(*)$, problem $\min _{x \in X} c^{T} x$ is equivalent to

$$
\text { Opt }=\min _{x, u}\left\{c^{T} x: A x+B u-b \in \mathbf{K}\right\}
$$

Definition: $\mathcal{K}$-representation of a function $f: \mathbb{R}^{n} \rightarrow \mathbb{R} \cup\{+\infty\}$ is a $\mathcal{K}$ representation of the epigraph of $f$ :

$$
\text { Epi }\{f\}:=\{(x, t): t \geq f(x)\}=\{x, t: \exists v: P x+p t+Q v-q \in \mathbf{K}\}, \quad \mathbf{K} \in \mathcal{K}
$$

$f$ is called $\mathcal{K}$-representable, if $f$ admits a $\mathcal{K}$-r. 
- A level set of a $\mathcal{K}-r$. function is $\mathcal{K}-r$.:

$$
\begin{gathered}
\text { Epi }\{f\}:=\{(x, t): t \geq f(x)\}=\{x, t: \exists v: P x+p t+Q u-q \in \mathbf{K}\} \\
\Rightarrow\{x: f(x) \leq c\}=\{x: \exists v: P x+Q u-[q-c p] \in \mathbf{K}\}
\end{gathered}
$$

- Minimization of a $\mathcal{K}-r$. function $f$ over a $\mathcal{K}-r$. set $X$ reduces to a conic program on a cone from $\mathcal{K}$ :

$$
\begin{gathered}
\left\{\begin{aligned}
x \in X \Leftrightarrow & \exists u: A x+B u-b \in \mathbf{K}_{X} \\
t \geq f(x) \Leftrightarrow & \exists v: P x+p t+Q v-q \in \mathbf{K}_{f}
\end{aligned}\right\} \\
\Downarrow \\
\quad \min _{x \in X} f(x) \\
\mathbb{1} \\
\min _{t, x, u, v}\{t:[A x+B u-b ; P x+p t+Q v-q] \in \underbrace{\mathbf{K}_{X} \times \mathbf{K}_{f}}_{\in \mathcal{K}}\}
\end{gathered}
$$


- Investigating "expressive abilities" of generic Magic conic problems reduces to answering the question

\section{What are $\mathcal{L P} / \mathcal{C} \mathcal{Q P} / \mathcal{S D} \mathcal{P}$-r. functions/sets?}

- "Built-in" restriction is convexity: $A \mathcal{K}$-representable set/function must be convex.

Good news: Convexity is, essentially, the only restriction: for all practical purposes, all convex sets/functions arising in applications are $\mathcal{S D P}$-r. Quite rich families of convex functions/sets are $\mathcal{L P} / \mathcal{C} \mathcal{Q P}$-r.

Note: Nonnegative orthants are direct products of (1-dimensional) Lorentz cones, and Lorentz cones are intersections of semidefinite cones and properly selected linear subspaces $\Rightarrow \mathcal{L P} \subset \mathcal{C Q P} \subset \mathcal{S D P}$. 
Let $\mathcal{K}$ be a family of regular cones closed w.r.t. taking direct products and passing from a cone $\mathrm{K}$ to its dual cone:

$$
\mathbf{K}_{*}=\{\lambda:\langle\lambda, \xi\rangle \geq 0 \forall \xi \in \mathbf{K}\}
$$

Note: $\mathbf{K}_{*}$ is regular cone provided $\mathrm{K}$ is so, and

$$
\left(\mathrm{K}_{*}\right)_{*}=\mathbf{K}
$$

Fact: $\mathcal{K}$-representable sets/functions admit fully algorithmic calculus:

- all basic convexity-preserving operations with functions/sets, as applied to $\mathcal{K}-r$. operands, produce $\mathcal{K}-r$. results, and the resulting $\mathcal{K}-$ r.'s are readily given by $\mathcal{K}$-r.'s of the operands.

- "Calculus rules" are independent of what $\mathcal{K}$ is.

$\Rightarrow$ Starting with "raw materials" (characteristic for $\mathcal{K}$ elementary $\mathcal{K}-r$. sets/functions) and applying calculus rules, we can recognize $\mathcal{K}$-representability and get explicit $\mathcal{K}-$ r.'s of sets/functions of interest. 


\section{“Calculus of $\mathcal{K}$-representability" basics:}

Sets: If $X_{1}, \ldots, X_{k}$ are $\mathcal{K}-$ r. sets, so are their

- intersections,

- images under affine mappings,
- direct products,

- inverse images under affine mappings.

Functions: If $f_{1}, \ldots, f_{k}$ are $\mathcal{K}$-r. functions, so are their

- linear combinations with nonnegative coefficients,

- superpositions with affine mappings.

Moreover, if $F, f_{1}, \ldots, f_{k}$ are $\mathcal{K}-r$. functions, so is the superposition $F\left(f_{1}(x), \ldots, f_{k}(x)\right)$

provided that $F$ is monotonically nondecreasing in its arguments.

More advanced convexity-preserving operations preserve $\mathcal{K}$-representability under (pretty mild!) regularity conditions. This includes

For sets: taking conic hulls and convex hulls of (finite) unions and passing from a set to its recessive cone, or polar, or support function

For functions: partial minimization, projective transformation, and taking Fenchel dual.

Note: Calculus rules are simple and algorithmic $\Rightarrow$ Calculus can be run in a compiler [used in $\mathrm{CVX}$ ]. 


\section{Illustration}

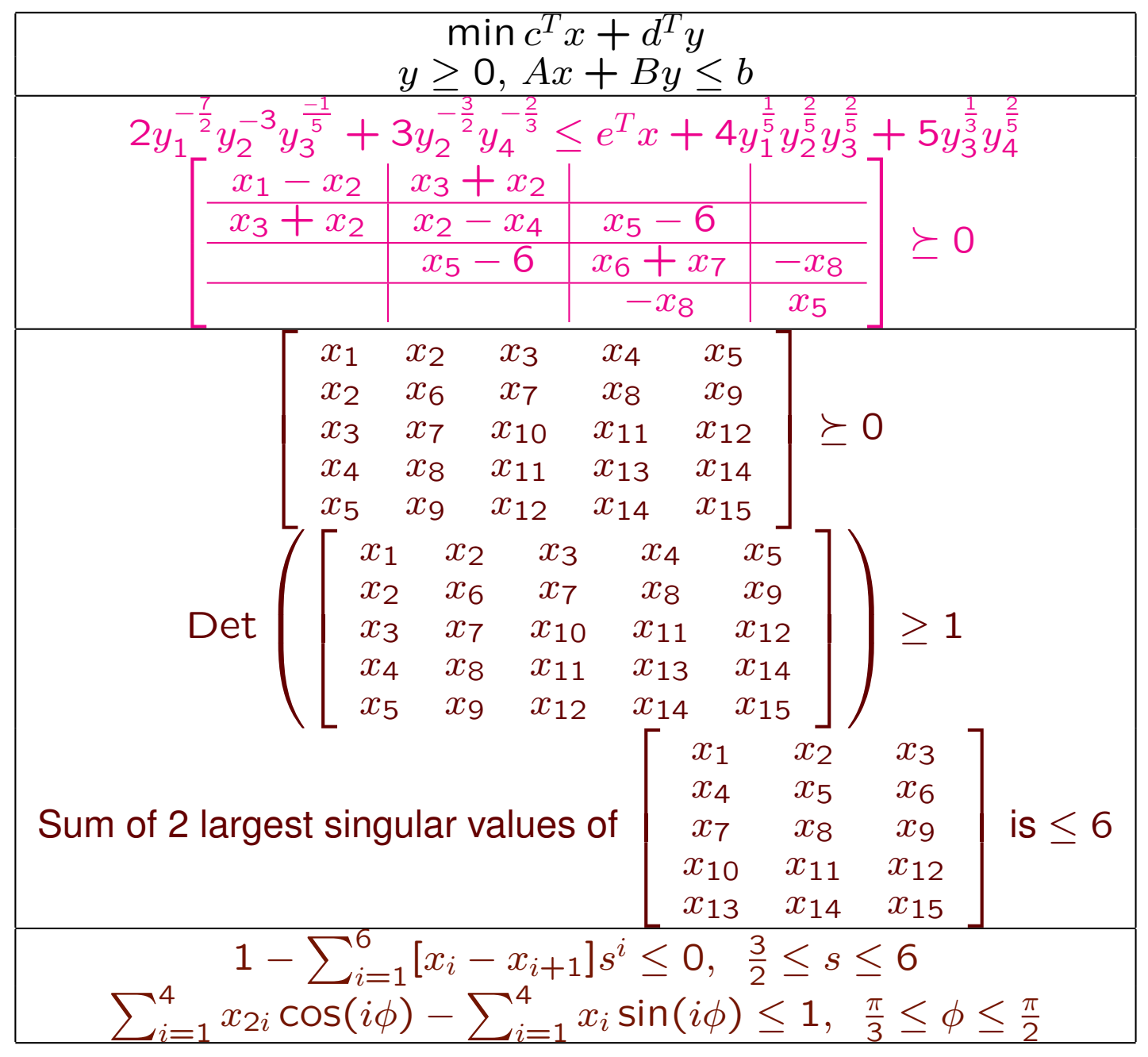

- blue part is in $\mathcal{L P}$

- blue-magenta part of the problem is in $\mathcal{C} \mathcal{Q P}$ (can be approximated, in a polynomial time, by $\mathcal{L P}$ ) - $\quad$ entire problem is in $\mathcal{S D} \mathcal{P}$

- reductions to $\mathcal{L P} / \mathcal{C} \mathcal{Q P} / \mathcal{S D P}$ are "fully algorithmic." 


\section{Conic Duality}

Conic Program admits nice Duality Theory completely similar to LP Duality. Primal problem:

$$
\begin{gathered}
\min _{x}\left\{c^{T} x: A x-b \geq_{\mathbf{K}} 0, R x=r\right\} \\
\mathbb{1} \text { [passing to primal slack } \xi=A x-b] \\
\min _{\xi}\left\{e^{T} \xi: \xi \in[\mathcal{L}-b] \cap \mathbf{K}\right\} \\
{\left[e: A^{T} e+R^{T} f=c \text { for some } f, \quad \mathcal{L}=\{A u: R u=0\}\right]}
\end{gathered}
$$

\section{Dual problem:}

$$
\begin{gathered}
\max _{y, z}\left\{b^{T} y: A^{T} y+R^{T} z=c, y \geq \mathbf{K}_{*} 0\right\} \\
\max _{y}\left\{b^{T} y: y \in\left[\mathcal{L}^{\perp}+e\right] \cap \mathbf{K}_{*}\right]
\end{gathered}
$$

$\left[\mathrm{K}_{*}:\right.$ cone dual to $\left.\mathbf{K}\right]$ 


\section{Primal problem:}

$$
\begin{gathered}
\min _{\xi}\left\{e^{T} \xi: \xi \in[\mathcal{L}-b] \cap \mathbf{K}\right\} \\
{\left[e: A^{T} e+R^{T} f=c \text { for some } f, \quad \mathcal{L}=\{A u: R u=0\}\right]}
\end{gathered}
$$

Dual problem:

$$
\begin{gathered}
\max _{y}\left\{b^{T} y: y \in\left[\mathcal{L}^{\perp}+e\right] \cap \mathbf{K}_{*}\right\} \\
{\left[\mathbf{K}_{*}: \text { cone dual to } \mathbf{K}\right]}
\end{gathered}
$$

\section{Note:}

- the dual problem is conic along with primal

- the duality is completely symmetric

- Cones from Magic Families are self-dual, so that the dual of a Linear/Conic Quadratic/Semidefinite program is of exactly the same type. 


\section{Deriving the dual problem}

Primal problem:

$$
\operatorname{Opt}(P)=\min _{x}\left\{\begin{array}{ll}
c^{T} x: & \begin{array}{l}
A_{i} x-b_{i} \in \mathbf{K}^{i}, i \leq m \\
R x=r
\end{array}
\end{array}\right\}
$$

Goal: find a systematic way to bound $\operatorname{Opt}(P)$ from below.

Observation: When $y_{i} \in \mathbf{K}_{*}^{i}$, the scalar inequality $y_{i}^{T} A_{i} x \geq y_{i}^{T} b_{i}$ is a consequence of the constraint $A_{i} x-b_{i} \in \mathbf{K}^{i}$.

If $z$ is a vector of the same dimension as $r$, the scalar inequality $z^{T} R x \geq z^{T} r$ is a consequence of the constraint $R x=r$. 
$\Rightarrow$ Whenever $y_{i} \in \mathbf{K}_{*}^{i}$ for all $i$ and $z$ is a vector of the same dimension as $r$, the scalar linear inequality

$$
\left[\sum_{i} A_{i}^{T} y_{i}+R^{T} z\right]^{T} x \geq \sum_{i} b_{i}^{T} y_{i}+r^{T} z
$$

is a consequence of the constraints in $(P)$

$\Rightarrow$ Whenever $y_{i} \in \mathbf{K}_{*}^{i}$ for all $i$ and $z$ is a vector of the same dimension as $r$ such that

$$
\sum_{i} A_{i}^{T} y_{i}+R^{T} z=c
$$

the quantity $\sum_{i} b_{i}^{T} y_{i}+r^{T} z$ is a lower bound on $\operatorname{Opt}(P)$.

Dual problem:

$$
\operatorname{Opt}(D)=\max _{y_{i}, z}\left\{\sum_{i} b_{i}^{T} y_{i}+r^{T} z: \begin{array}{l}
y_{i} \in \mathbf{K}_{*}^{i}, i \leq m \\
\sum_{i} A_{i}^{T} y_{i}+R^{T} z=c
\end{array}\right\}
$$

is exactly the problem of maximizing this lower bound on $\operatorname{Opt}(P)$. 
Definition A conic problem

$$
\min _{x}\left\{\begin{array}{ll}
c^{T} x: \begin{array}{l}
A_{i} x-b_{i} \in \mathbf{K}^{i}, i \leq m \\
A x \geq b \\
R x=r
\end{array}
\end{array}\right\}
$$

is called strictly feasible, if there exists a feasible solution $\bar{x}$ where all conic and $\leq$ constraints are strictly satisfied:

$$
A_{i} \bar{x}-b_{i} \in \operatorname{int} \mathbf{K}^{i} \forall i \& A \bar{x}>b .
$$

It is called essentially strictly feasible, if there exists a feasible solution $\bar{x}$ where all non-polyhedral constraints are strictly satisfied:

$$
A_{i} \bar{x}-b_{i} \in \operatorname{int} \mathbf{K}^{i} \forall i \text {. }
$$


Conic Duality Theorem Consider a conic problem

$$
\operatorname{Opt}(P)=\min _{x}\left\{\begin{array}{ll}
c^{T} x: \begin{array}{l}
A_{i} x-b_{i} \in \mathbf{K}^{i}, i \leq m \\
R x=r
\end{array}
\end{array}\right\}
$$

along with its dual

Then

$$
\operatorname{Opt}(D)=\max _{y_{i}, z}\left\{\sum_{i} b_{i}^{T} y_{i}+r^{T} z: \begin{array}{l}
y_{i} \in \mathbf{K}_{*}^{i}, i \leq m \\
\sum_{i} A_{i}^{T} y_{i}+R^{T} z=c
\end{array}\right\}
$$

- [Symmetry] Duality is symmetric: the dual problem is conic, and its dual is (equivalent to) the primal problem;

- [Weak duality] One has Opt $(D) \leq \operatorname{Opt}(P)$;

- [Strong duality] Let one of the problems be essentially strictly feasible and bounded. Then the other problem is solvable, and

$$
\operatorname{Opt}(D)=\operatorname{Opt}(P) \text {. }
$$

In particular, if both problems are essentially strictly feasible, both are solvable with equal optimal values. 


$$
\begin{gathered}
\min _{x}\left\{\begin{array}{c}
c^{T} x: \begin{array}{l}
A_{i} x-b_{i} \in \mathbf{K}^{i}, i \leq m \\
R x=r
\end{array} \\
\uparrow \downarrow
\end{array}\right\} \\
\max _{y_{i}, z}\left\{\sum_{i} b_{i}^{T} y_{i}+r^{T} z: \begin{array}{l}
y_{i} \in \mathbf{K}_{*}^{i}, i \leq m \\
\sum_{i} A_{i}^{T} y_{i}+R^{T} z=c
\end{array}\right\}
\end{gathered}
$$

\section{Conic Programming Optimality Conditions}

Let both $(P)$ and $(D)$ be essentially strictly feasible. Then a pair $\left(x,\left[\left\{y_{i}\right\}, z\right]\right)$ of primal and dual feasible solutions is comprised of optimal solutions to the respective problems if and only if

$$
\text { [Zero Duality Gap] DualityGap }\left(x,\left[\left\{y_{i}\right\}, z\right]\right):=c^{T} x-\left[\sum_{i} b_{i}^{T} y_{i}+r^{T} z\right]=0
$$

Indeed,

$$
\operatorname{DualityGap}\left(x,\left[\left\{y_{i}\right\}, z\right]\right)=\underbrace{\left[c^{T} x-\operatorname{Opt}(P)\right]}_{\geq 0}+\underbrace{\left[\operatorname{Opt}(D)-\left[\sum_{i} b_{i}^{T} y_{i}+r^{T} z\right]\right]}_{\geq 0}
$$

\section{[Complementary Slackness] $\quad\left[A_{i} x-b_{i}\right]^{T} y_{i}=0, i \leq m$}

Indeed,

$$
\begin{aligned}
\sum_{i} \underbrace{\left[A_{i} x-b_{i}\right]^{T} y_{i}}_{\geq 0} & =\left[\sum_{i} A_{i}^{T} y_{i}\right] x-\sum_{i} b_{i}^{T} y_{i}=\underbrace{\left[c-R^{T} z\right]^{T} x}_{=c^{T} x-r^{T} z}-\sum_{i} b_{i}^{T} y_{i}=c^{T} x-\left[\sum_{i} b_{i}^{T} y_{i}+r^{T} z\right] \\
& =\text { DualityGap }\left(x,\left[\left\{y_{i}\right\}, z\right]\right)
\end{aligned}
$$


- Conic Duality, same as the LP one, is

- fully algorithmic: to write down the dual, given the primal, is a purely mechanical process

- fully symmetric: the dual problem "remembers" the primal one

- Cf. Lagrange Duality:

$$
\begin{gathered}
\min _{x}\left\{f(x): g_{i}(x) \leq 0, i=1, \ldots, m\right\} \\
\Downarrow \\
\max _{y \geq 0} \underline{L}(y) \\
{\left[\underline{L}(y)=\min _{x}\left\{f(x)+\sum_{i} y_{i} g_{i}(x)\right\}\right]}
\end{gathered}
$$

- Dual "exists in the nature", but is given implicitly; its objective, typically, is not available in a closed form

- Duality is asymmetric: given $\underline{L}(\cdot)$, we, typically, cannot recover $f$ and $g_{i} \ldots$ 


\section{Developing Tools, Optimization: Schur Complement Lemma}

Lemma Symmetric block matrix $\left[\begin{array}{c|c}P & S^{T} \\ \hline S & R\end{array}\right]$ with $R \succ 0$ is positive semidefinite if and only if the matrix $P-S^{T} R^{-1} S$ is so.

Proof: $\left[\begin{array}{c|c}P & S^{T} \\ \hline S & R\end{array}\right] \succeq 0$ iff

$$
\begin{aligned}
0 & \leq \min _{u, v}\left[u^{T} P u+2 u^{T} S^{T} v+v^{T} R v\right] \\
& =\min _{u}[\underbrace{\min _{v}\left[u^{T} P u+2 u^{T} S^{T} v+v^{T} R v\right]}_{\text {achieved when } v=-R^{-1} S u}] \\
& =\min _{u} u^{T}\left[P-S^{T} R^{-1} S\right] u .
\end{aligned}
$$




\section{Developing Tools, Statistics: \\ Bayesian Risk and Gauss-Markov Theorem}

An alternative to the worst-case risk (the worst over the signals of interest, performance of a statistical procedure) is average performance, with the average taken over some prior probability distribution on the signals.

In the problem of $\|\cdot\|_{2}$-recovering $B x$ via noisy observation

$$
\omega=A x+\sigma \xi, \xi \sim P
$$

this alternative reads as follows:

(!) Given a probability distribution $\Pi$ of signal $x \in \mathbb{R}^{n}$, find an estimate $\widehat{x}(\cdot)$ which minimizes

$$
\operatorname{Risk}^{2}[\widehat{x} \mid \Pi]:=\int_{\Pi}\left\{\int_{\mathbb{R}^{m}}\|B x-\widehat{x}(A x+\sigma \xi)\|_{2}^{2} P(d \xi)\right\} \Pi(d x)
$$

- the average, over the distribution $\Pi$ of signals $x$, of expected $\|\cdot\|_{2}^{2}$ recovery error of $B x$ via observation $A x+\sigma \xi$. 
(!) Given a probability distribution $\Pi$ of signal $x \in \mathbb{R}^{n}$, find an estimate $\widehat{x}(\cdot)$ which minimizes

$$
\operatorname{Risk}^{2}[\widehat{x} \mid \Pi]:=\int_{\Pi}\left\{\int_{\mathbb{R}^{m}}\|B x-\widehat{x}(A x+\sigma \xi)\|_{2}^{2} P(d \xi)\right\} \Pi(d x)
$$

- the average, over the distribution $\Pi$ of signals $x$, of expected $\|\cdot\|_{2}^{2}$ recovery error of $B x$ via observation $A x+\sigma \xi$.

- $Q$ : joint distribution of $(x, \omega=A x+\sigma \xi)$ induced by distributions $\Pi$ and $P$ of independent r.v.'s $x, \xi$

- $R_{\omega}$ : conditional distribution of $x$, given $\omega$ of $x$,

- $W$ : marginal distribution of $\omega$.

We have

$$
\begin{aligned}
\operatorname{Risk}^{2}[\widehat{x} \mid \Pi] & =\int_{\mathbb{R}_{x}^{n} \times \mathbb{R}_{\omega}^{m}}\|B x-\widehat{x}(\omega)\|_{2}^{2} Q(d x, d \omega) \\
& =\int_{\mathbb{R}^{m}}\left\{\int_{\mathbb{R}^{n}}\|B x-\widehat{x}(\omega)\|_{2}^{2} R_{\omega}(d x)\right\} W(d \omega)
\end{aligned}
$$


Evident Fact: Assuming that a probability distribution $S$ on $\mathbb{R}^{n}$ possesses finite second moments, one has

$$
\begin{gathered}
\min _{c \in \mathbb{R}^{\nu}} \int_{\mathbb{R}^{n}}\|B x-c\|_{2}^{2} S(d x)=\int_{\mathbb{R}^{n}}\left\|B x-c_{*}\right\|_{2} S(d x), \\
c_{*}=\int_{\mathbb{R}^{n}} B x S(d x) .
\end{gathered}
$$

$\Rightarrow$ An optimal, in terms of $\operatorname{Risk}^{2}[\widehat{x} \mid \Pi]$, estimate $\widehat{x}(\cdot)$ of $B x$ via $\omega=A x+\sigma \xi$ is

$$
\widehat{x}_{*}(\omega)=\int_{\mathbb{R}^{n}} B x R_{\omega}(d x)
$$

Gauss-Markov Theorem [Normal Correlation Theorem] Let $x \in \mathbb{R}^{n}$ and $\xi \in$ $\mathbb{R}^{m}$ be independent zero mean Gaussian random vectors. Assuming $\sigma>0$ and the covariance matrix of $\xi$ to be positive definite, an optimal solution $\widehat{x}(\cdot)$ to the risk minimization problem

$$
\min _{\widehat{x}(\cdot)} \mathbf{E}_{x, \xi}\left\{\|B x-\widehat{x}(A x+\sigma \xi)\|_{2}^{2}\right\}
$$

exists and can be selected as a linear function of $\omega=A x+\sigma \xi$. 
Under the premise of Gauss-Markov Theorem, with $x \sim \mathcal{N}(0, Q)$ and $\xi \sim$ $\mathcal{N}\left(0, I_{m}\right)$, direct computation shows that

$$
\begin{aligned}
\widehat{x}_{*}(\omega) & =\left[\left[\sigma^{2} I_{m}+A Q A^{T}\right]^{-1} A Q B^{T}\right] \omega \\
\operatorname{Risk}^{2}\left[\widehat{x}_{*} \mid \mathcal{N}(0, Q)\right] & =\operatorname{Tr}\left(B Q B^{T}-B Q A^{T}\left[\sigma^{2} I_{m}+A Q A^{T}\right]^{-1} A Q B^{T}\right)
\end{aligned}
$$




\section{Optimizing linear estimates}

Situation: "In the nature" there exists a signal $x$ known to belong to a given convex compact set $\mathcal{X} \subset \mathbb{R}^{n}$. We observe corrupted by noise affine image of the signal:

$$
\omega=A x+\sigma \xi \in \Omega=\mathbb{R}^{m}
$$

where

- $A$ is a given $m \times n$ sensing matrix

- $\xi$ is a random noise

Goal: To recover the image $B x$ of $x$ where

- $B$ : given $\nu \times n$ matrix.

Risk of a candidate estimate $\widehat{x}(\cdot): \Omega \rightarrow \mathbb{R}^{\nu}$ is

$$
\operatorname{Risk}[\widehat{x} \mid \mathcal{X}]=\sup _{x \in \mathcal{X}} \sqrt{\mathbf{E}_{\xi}\left\{\|B x-\widehat{x}(A x+\sigma \xi)\|_{2}^{2}\right\}}
$$


Assumption on noise: $\xi$ is zero mean with unit covariance matrix.

$\Rightarrow$ The risk of a linear estimate $\widehat{x}_{H}(\omega)=H^{T} \omega$ is given by

$$
\begin{aligned}
\operatorname{Risk}^{2}\left[\widehat{x}_{H} \mid \mathcal{X}\right] & =\max _{x \in \mathcal{X}} \mathbf{E}_{\xi}\left\{\left\|\left[B-H^{T} A\right] x-\sigma H^{T} \xi\right\|_{2}^{2}\right\} \\
& =\max _{x \in \mathcal{X}}\left\{\left\|\left[B-H^{T} A\right] x\right\|_{2}^{2}+\sigma^{2} \mathbf{E}_{\xi}\left\{\operatorname{Tr}\left(H^{T} \xi \xi^{T} H\right)\right\}\right\} \\
& =\sigma^{2} \operatorname{Tr}\left(H^{T} H\right)+\underbrace{\max _{x \in \mathcal{X}} x^{T}\left[B-H^{T} A\right]^{T}\left[B-H^{T} A\right] x}_{\Psi(H)} .
\end{aligned}
$$

Note: $\Psi$ is convex $\Rightarrow$ building the minimum risk linear estimate reduces to solving convex minimization problem

$$
\text { Opt }=\min _{H}\left[\Psi(H)+\sigma^{2} \operatorname{Tr}\left(H^{T} H\right)\right] .
$$

However: Convex function $\psi$ is given implicitly and can be difficult to compute, making $(*)$ difficult as well. 


$$
\begin{aligned}
\text { Opt } & =\min _{H}\left[\sigma^{2} \operatorname{Tr}\left(H^{T} H\right)+\Psi(H)\right] \\
\Psi(H) & =\max _{x \in \mathcal{X}} x^{T}\left[B-H^{T}\right]^{T}\left[B-H^{T} A\right] x
\end{aligned}
$$

Fact: Basically, the only cases when $(*)$ is known to be easy are those when

- $\mathcal{X}$ is given as a convex hull of finite set of moderate cardinality

- $\mathcal{X}$ is an ellipsoid.

When $\mathcal{X}$ is a box, computing $\Psi$ is NP-hard...

- When $\psi$ is difficult to compute, we can to replace $\psi$ in the design problem (*) with an efficiently computable convex upper bound $\Psi^{+}(H)$.

We are about to consider a family of sets $\mathcal{X}$ - ellitopes - for which reasonably tight bounds $\psi^{+}$of desired type are available. 
A basic ellitope is a set $\mathcal{Y} \subset \mathbb{R}^{N}$ given as

$$
\mathcal{Y}=\left\{y \in \mathbb{R}^{N}: \exists t \in \mathcal{T}: y^{T} S_{k} y \leq t_{k}, k \leq K\right\}
$$

where

- $S_{k} \succeq 0$ are positive semidefinite matrices with $\sum_{k} S_{k} \succ 0$

- $\mathcal{T}$ is a convex compact subset of $K$-dimensional nonnegative orthant $\mathbb{R}_{+}^{K}$ such that

- $\mathcal{T}$ contains some positive vectors

- $\mathcal{T}$ is monotone: if $0 \leq t^{\prime} \leq t$ and $t \in \mathcal{T}$, then $t^{\prime} \in \mathcal{T}$ as well.

An ellitope $\mathcal{X}$ is linear image of a basic ellitope:

$$
\mathcal{X}=\left\{x \in \mathbb{R}^{n}: \exists y \in \mathbb{R}^{N}, t \in \mathcal{T}: x=F y, y^{T} S_{k} y \leq t_{k}, k \leq K\right\}
$$

- $F$ is a given $n \times N$ matrix,

Note: Every ellitope is a symmetric w.r.t. the origin convex compact set. 


\section{Examples of basic ellitopes}

A. Ellipsoid centered at the origin

$$
(K=1, \mathcal{T}=[0 ; 1])
$$

B. (Bounded) intersection of $K$ ellipsoids/elliptic cylinders centered at the origin

$$
\left(\mathcal{T}=\left\{t \in \mathbb{R}^{K}: 0 \leq t_{k} \leq 1, k \leq K\right\}\right)
$$

C. Box $\left\{x \in \mathbb{R}^{n}:-1 \leq x_{i} \leq 1\right\}$

$$
\left(\mathcal{T}=\left\{t \in \mathbb{R}^{n}: 0 \leq t_{k} \leq 1, k \leq K=n\right\}, x^{T} S_{k} x=x_{k}^{2}\right)
$$

$\mathbf{C}^{\prime}$. Dual ball of Vasershtein norm $\mathcal{B}_{*}=\left\{u \in \mathbb{R}^{n}:\left|u_{i}-u_{j}\right| \leq d_{i j}, i, j \leq n\right\}$

$\mathcal{B}_{*}=\left\{u \in \mathbb{R}^{n}: u^{T} S_{i j} u \leq 1,1 \leq i \leq j \leq n\right\}, S_{i j}= \begin{cases}d_{i j}^{-2}\left[e_{i}-e_{j}\right]\left[e_{i}-e_{j}\right]^{T}, & i<j \leq n, \\ 4\left[\max _{i j} d_{i j}\right]^{-2} e_{i} e_{i}^{T}, & i=j \leq n\end{cases}$ where $e_{i}, e_{j}$ are canonic orths of $\mathbb{R}^{n}$.

D. $\ell_{p}$-ball $\mathcal{X}=\left\{x \in \mathbb{R}^{n}:\|x\|_{p} \leq 1\right\}$ with $p \geq 2$

$$
\left(\mathcal{T}=\left\{t \in \mathbb{R}_{+}^{n}:\|t\|_{p / 2} \leq 1\right\}, x^{T} S_{k} x=x_{k}^{2}, k \leq K=n\right)
$$


Ellitopes admit fully algorithmic calculus:

if $\mathcal{X}_{i}, 1 \leq i \leq I$, are ellitopes, so are their

- intersection $\bigcap_{i} \mathcal{X}_{i}$

- direct product $\mathcal{X}_{1} \times \ldots \times \mathcal{X}_{I}$

- arithmetic sum $\mathcal{X}_{1}+\ldots+\mathcal{X}_{I}$

- linear images $\left\{A x: x \in \mathcal{X}_{i}\right\}$

- inverse linear images $\left\{y: A y \in \mathcal{X}_{i}\right\}$ under linear embeddings $A$ 


\section{Observation: Let}

$$
\mathcal{X}=\left\{x: \exists(t \in \mathcal{T}, y): x=F y, y^{T} S_{k} y \leq t_{k}, k \leq K\right\}
$$

be an ellitope. Given a quadratic form $x^{T} W x, W \in \mathbf{S}^{n}$, we have

$$
\max _{x \in \mathcal{X}} x^{T} W x \leq \min _{\lambda}\left\{\phi_{\mathcal{T}}(\lambda): \lambda \geq 0, \sum_{k=1}^{K} \lambda_{k} S_{k} \succeq F^{T} W F\right\}
$$

where

$$
\phi_{\mathcal{T}}(\lambda)=\max _{t \in \mathcal{T}} t^{T} \lambda
$$

is the support function of $\mathcal{T}$.

Indeed, assume that $\lambda \geq 0$ and $F^{T} W F \preceq \sum_{k} \lambda_{k} S_{k}$. By definition of the ellitope, if $x \in \mathcal{X}$,

$$
\exists(t \in \mathcal{T}, y): y^{T} S_{k} y \leq t_{k} \quad \forall k \leq K, x=F y
$$

We have $x^{T} W x=y^{T} F^{T} W F y$, but

$$
y^{T} F^{T} W F y \leq \sum_{k} \lambda_{k} y^{T} S_{k} y \leq \sum_{k} \lambda_{k} t_{k} \leq \max _{t \in \mathcal{T}} t^{T} \lambda=\phi_{\mathcal{T}}(\lambda),
$$

and we conclude that

$$
x^{T} W x \leq \phi_{\mathcal{T}}(\lambda)
$$




\section{Where does it come from: Semidefinite Relaxation}

$$
\Psi=\max _{x \in \mathcal{X}} x^{T} W x, \quad \mathcal{X}=\left\{x: \exists(t \in \mathcal{T}, y): x=F y, y^{T} S_{k} y \leq t_{k}, k \leq K\right\}
$$

- Let us denote $X=x x^{T}$, then $(*)$ can be rewritten equivalently:

$\max \left\{\operatorname{Tr}(W X): \begin{array}{c}\exists\left(t \in \mathcal{T}, Q \in \mathbf{S}_{n}\right): X=F Q F^{T}, \operatorname{Tr}\left(S_{k} Q\right) \leq t_{k}, k \leq K, \\ Q \succeq 0, \operatorname{Rank}(Q)=1\end{array}\right\}$

Then $\Psi \leq \Psi$ where

$\bar{\Psi}=\max _{Q \in \mathcal{Q}} \operatorname{Tr}(\underbrace{F^{T} W F}_{=: V} Q), \mathcal{Q}=\left\{Q \succeq 0: \exists t \in \mathcal{T}: \operatorname{Tr}\left(S_{k} Q\right) \leq t_{k}, k \leq K\right\}$

Note that $\left(*_{1}\right)$ is a convex program, it is instructive to write it as Conic Programming. 
- Conic representation of constraint $t \in \mathcal{T}$.

- Let

$$
\mathcal{T}^{+}=\left\{[t ; 1] \in \mathbb{R}^{K+1}: t \in \mathcal{T}\right\}, \quad \mathbf{T}=\left\{\lambda s \in \mathbb{R}^{K+1}, \quad \lambda \geq 0, s \in \mathcal{T}^{+}\right\} .
$$

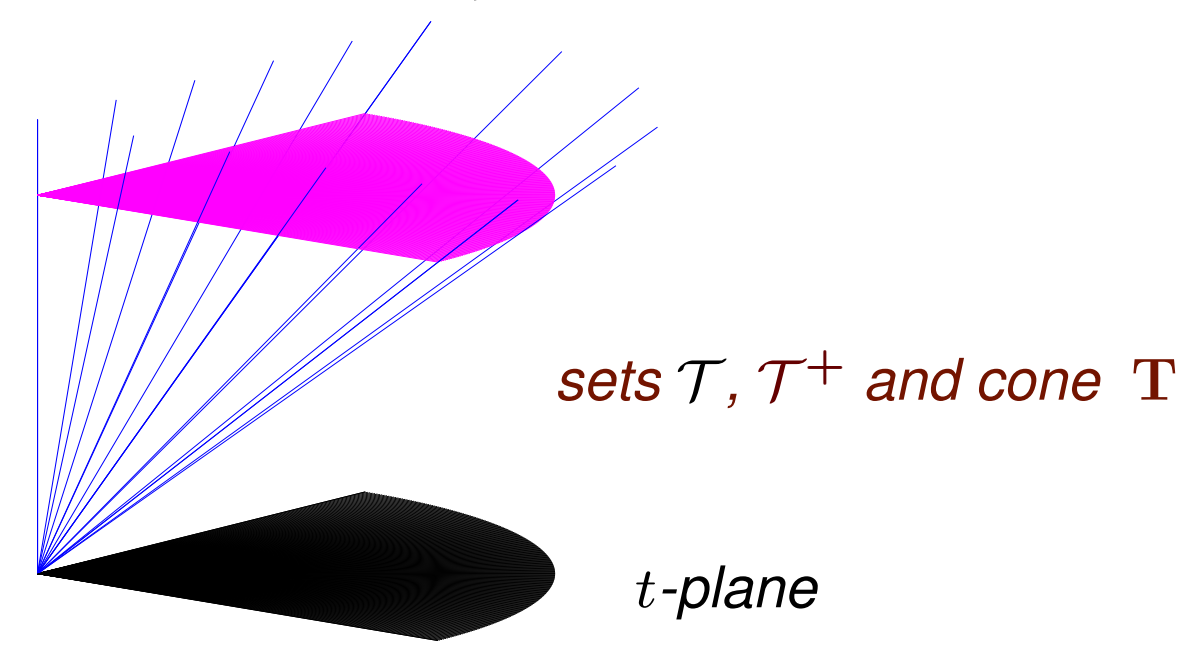

- $\mathrm{T}$ is a regular cone (since $\mathcal{T}$ is a convex compact set with a nonempty interior)

- $\mathcal{T}=\{t:[t ; 1] \in \mathbf{T}\}$ 
The dual cone to $\mathbf{T}=\mathbb{R}_{+} \times\{[t ; 1]: t \in \mathcal{T}\}$ is

$$
\left\{[y ; s] \in \mathbb{R}^{k+1}: s \geq \phi_{\mathcal{T}}(-g)\right\}
$$

Indeed, if $\mathbf{T}_{*}$ is the cone dual to $\mathbf{T}$,

$$
\begin{aligned}
\left\{[y ; s] \in \mathbf{T}_{*}\right\} & \Leftrightarrow\left\{y^{T} t+s \tau \geq 0 \forall[t ; \tau] \in \mathbf{T}\right\} \\
& \Leftrightarrow\left\{y^{T} t+s \geq 0 \forall t:[t ; 1] \in \mathbf{T}\right\} \Leftrightarrow s \geq-y^{T} t \forall t \in \mathcal{T} \Leftrightarrow s \geq \max _{t \in \mathcal{T}}[-y]^{T} t \\
& \Leftrightarrow\left\{s \geq \phi_{\mathcal{T}}(-y)\right\}
\end{aligned}
$$

Bottom line: We have

$$
\bar{\Psi}=\max _{Q, t}\left\{\operatorname{Tr}(V Q):[t ; 1] \in \mathbf{T}, Q \succeq 0, \operatorname{Tr}\left(S_{k} Q\right) \leq t_{k} \forall k\right\}
$$

Question: What is the conic dual to $\left(*_{1}\right)$ ? 
- To pass from $\left(*_{1}\right)$ to the dual problem we associate Lagrange multiplyers to the constraints:

$$
\underbrace{[t ; 1] \in \mathbf{T}}_{[g ; s] \in \mathbf{T}_{*}}, \underbrace{Q \succeq 0}_{P \succeq 0}, \underbrace{t_{k}-\operatorname{Tr}\left(Q S_{k}\right) \geq 0}_{\lambda_{k} \geq 0} \forall k \leq K
$$

- Taking inner products of the constraints with Lagrange multipliers and summing up the results, we get the consequence of the constraints of $\left(*_{1}\right)$ :

$$
\begin{gathered}
g^{T} t+s \cdot 1+\operatorname{Tr}(P Q)+\sum_{k} \lambda_{k}\left[t_{k}-\operatorname{Tr}\left(Q S_{k}\right)\right] \geq 0 \\
\downarrow \uparrow \\
\operatorname{Tr}\left(\left[\sum_{k} \lambda_{k} S_{k}-P\right] Q\right)-[g+\lambda]^{T} t \leq s, \quad\left[s \geq \phi_{\mathcal{T}}(-g), P \succeq 0, \lambda \geq 0\right]
\end{gathered}
$$


- To get the dual problem, we impose on Lagrange multiplies the requirement that the body of the aggregated constraint, as a function of $Q, t$, is identically equal to the objective $\operatorname{Tr}(V Q)$ of $\left(*_{1}\right)$ :

$$
\begin{aligned}
& \bar{\Psi}=\min _{g, s, \lambda, P}\left\{s: \begin{array}{l}
g+\lambda=0, \sum_{k} \lambda_{k} S_{k}-P=V \\
s \geq \phi_{\mathcal{T}}(-g), P \succeq 0, \lambda \geq 0
\end{array}\right\} \\
& \text { I } \\
& \bar{\Psi}=\min _{g, s, \lambda, P}\left\{s: \begin{array}{l}
g=-\lambda, \sum_{k} \lambda_{k} S_{k}-P=V \\
s \geq \phi_{\mathcal{T}}(-g), P \succeq 0, \lambda \geq 0
\end{array}\right\} \\
& =\min _{s, \lambda}\left\{\begin{array}{ll}
s: \begin{array}{l}
\sum_{k} \lambda_{k} S_{k} \succeq V \\
s \geq \phi_{\mathcal{T}}(\lambda), \lambda \geq 0
\end{array}
\end{array}\right\} \\
& =\min _{\lambda}\{\phi_{\mathcal{T}}(\lambda): \underbrace{\sum_{k} \lambda_{k} S_{k} \succeq V}_{\sum_{k} \lambda_{k} S_{k} \succeq F^{T} W F}, \lambda \geq 0\}
\end{aligned}
$$




$$
\mathcal{X}=\left\{x: \exists(t \in \mathcal{T}, y): x=F y, y^{T} S_{k} y \leq t_{k}, k \leq K\right\}
$$

Corollary Let $\mathcal{X}$ be the ellitope $(*)$. Then the function

$$
\Psi(H)=\max _{x \in \mathcal{X}} x^{T}\left[\left(B^{T}-A^{T} H\right)\left(B-H^{T} A\right)\right] x
$$

can be upper-bounded: $\Psi(H) \leq \bar{\Psi}(H)$ where

$$
\begin{aligned}
& \bar{\Psi}(H):=\min _{\lambda}\left\{\phi_{\mathcal{T}}(\lambda): \lambda \geq 0, F^{T}\left[B^{T}-A^{T} H\right]\left[B-H^{T} A\right] F \preceq \sum_{k} \lambda_{k} S_{k}\right\} \\
& \text { [Schur Complement Lemma] } \\
& =\min _{\lambda}\left\{\phi_{\mathcal{T}}(\lambda): \lambda \geq 0,\left[\begin{array}{c|c}
\sum_{k} \lambda_{k} S_{k} & F^{T}\left[B^{T}-A^{T} H\right] \\
\hline\left[B-H^{T} A\right] F & I_{\nu}
\end{array}\right] 0\right\}
\end{aligned}
$$

The function $\bar{\Psi}(H)$ is real-valued and convex, and is efficiently computable whenever $\phi_{\mathcal{T}}$ is so, that is, whenever $\mathcal{T}$ is computationally tractable. 
Bottom line: Given matrices $A \in \mathbb{R}^{m \times n}, B \in \mathbb{R}^{\nu \times n}$ and an ellitope

$$
\mathcal{X}=\left\{x: \exists(t \in \mathcal{T}, y): x=F y, y^{T} S_{k} y \leq t_{k}, k \leq K\right\}
$$

contained in $\mathbb{R}^{n}$, consider the convex optimization problem

$$
\begin{aligned}
& \text { Opt }=\min _{H, \lambda}\left\{\phi_{\mathcal{T}}(\lambda)+\sigma^{2} \operatorname{Tr}\left(H^{T} H\right): \lambda \geq 0,\left[\begin{array}{c|c}
\sum_{k} \lambda_{k} S_{k} & F^{T}\left[B^{T}-A^{T} H\right] \\
{\left[B-H^{T} A\right] F} & I_{\nu}
\end{array}\right] 0\right\} \\
& {\left[\phi_{\mathcal{T}}(\lambda)=\max _{t \in \mathcal{T}} t^{T} \lambda: \text { support function of } \mathcal{T}\right]}
\end{aligned}
$$

Assuming the noise $\xi$ in observation $\omega=A x+\sigma \xi$ zero mean with unit covariance matrix, the risk of the linear estimate $\widehat{x}_{H_{*}}(\cdot)$ induced by the optimal solution $H_{*}$ to the problem (this solution clearly exists provided $\sigma>0$ ) satisfies the risk bound

$$
\operatorname{Risk}\left[\widehat{x}_{H_{*}} \mid \mathcal{X}\right] \leq \sqrt{\mathrm{Opt}} .
$$

What is ahead: We are about to prove that in the case of $\xi \sim \mathcal{N}\left(0, I_{m}\right)$, $\sqrt{\mathrm{Opt}}$ is "nearly" the same as the ideal minimax risk

$$
\text { RiskOpt }=\inf _{\widehat{x}(\cdot)} \operatorname{Risk}[\widehat{x} \mid \mathcal{X}],
$$

where inf is taken w.r.t. all (not necessarily linear) estimates $\widehat{x}(\cdot)$. 


\section{How it works: Inverse Heat Equation}

Square plate is heated at time 0 and is rest to cool; the temperature at the plate's boundary is kept 0 all the time.

Given given noisy measurements, taken along $m$ points, of plate's temperature at time $t_{1}$, we want to recover distribution of temperature at time a given time $t_{0}, 0<t_{0}<t_{1}$.

The model: The temperature field $u(t ; p, q)$ evolves according to Heat Equation

$$
\begin{gathered}
\frac{\partial}{\partial t} u(t ; p, q)=\left[\frac{\partial^{2}}{\partial p^{2}}+\frac{\partial^{2}}{\partial q^{2}}\right] u(t ; p, q), t \geq 0,(p, q) \in S \\
\bullet t: \text { time } \bullet S=\{(p, q),-1 \leq p, q \leq 1\}: \text { the plate }
\end{gathered}
$$

with boundary conditions $\left.u(t ; p, q)\right|_{(p, q) \in \partial S} \equiv 0$.

- It is convenient to represent $u(t ; p, q)$ by its expansion

$$
\begin{aligned}
& u(t ; p, q)=\sum_{k, \ell} x_{k \ell}(t) \phi_{k}(p) \phi_{\ell}(q) \text {, }
\end{aligned}
$$

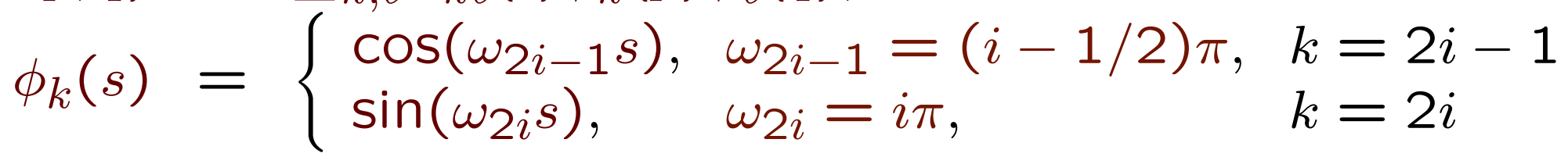

Note: $\phi_{k}(s)$ are harmonic oscillations vanishing at $s= \pm 1$. 


$$
\begin{aligned}
& u(t ; p, q)=\sum_{k, \ell} x_{k \ell}(t) \phi_{k}(p) \phi_{\ell}(q),
\end{aligned}
$$

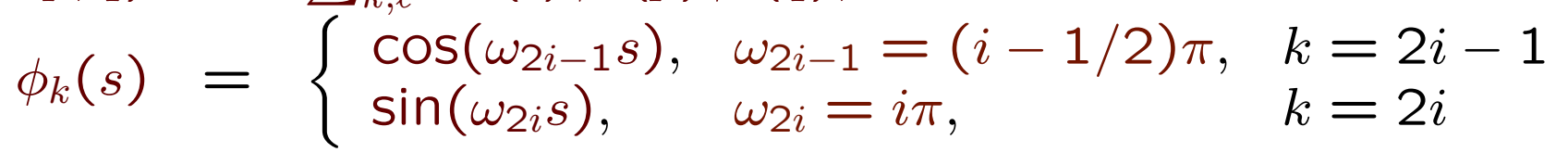

- $\left\{\phi_{k \ell}(p, q)=\phi_{k}(p) \phi_{\ell}(q)\right\}_{k, \ell}$ form an orthonormal system in $L_{2}(S)$

- $\phi_{k \ell}(\cdot)$ meet the boundary conditions

$$
\left.\phi_{k \ell}(p, q)\right|_{(p, q) \in \partial S}=0
$$

- in terms of the coefficients $x_{k \ell}(t)$, the Heat Equation becomes

$$
\frac{d}{d t} x_{k \ell}(t)=-\left[\omega_{k}^{2}+\omega_{\ell}^{2}\right] x_{k \ell}(t) \Rightarrow x_{k \ell}(t)=e^{-\left[\omega_{k}^{2}+\omega_{\ell}^{2}\right] t} x_{k \ell}(0) .
$$

We select integer discretization parameter $N$ and

- restrict $(*)$ to terms with $1 \leq k, \ell \leq 2 N-1$

- discretize the spatial variable $(p, q)$ to reside in the grid

$$
G_{N}=\left\{P_{i j}=\left(p_{i}, p_{j}\right)=\left(\frac{i}{N}-1, \frac{j}{N}-1\right), 1 \leq i, j \leq 2 N-1\right\}
$$

Note: Restricting functions $\phi_{k \ell}(\cdot, \cdot), 1 \leq k, \ell \leq 2 N-1$ on grid $G_{N}$, we get orthogonal basis in $\mathbb{R}^{(2 N-1) \times(2 N-1)}$. 
We arrive at the model as follows:

- The signal $x$ underlying observation is

$$
x=\left\{x_{k \ell}:=x_{k \ell}\left(t_{0}\right), 1 \leq k, \ell \leq 2 N-1\right\} \in \mathbb{R}^{(2 N-1) \times(2 N-1)}
$$

- The observation is

$$
\begin{aligned}
\omega & =A x+\sigma \xi \in \mathbb{R}^{m}, \quad \xi \sim \mathcal{N}\left(0, I_{m}\right) \\
{[A x]_{\nu} } & =\sum_{k, \ell=1}^{2 N-1} x_{k \ell} \mathrm{e}^{-\left[\omega_{k}^{2}+\omega_{\ell}^{2}\right]\left[t_{1}-t_{0}\right]} \phi_{k}\left(p_{i(\nu)}\right) \phi_{\ell}\left(p_{j(\nu)}\right) \\
& \bullet\left(p_{i(\nu)}, p_{j(\nu)}\right) \in S, \quad 1 \leq \nu \leq m: \text { measurement points }
\end{aligned}
$$

- We want to recover the restriction $B x$ of $u\left(t_{0} ; p, q\right)$ to some grid, say, square grid

$$
G_{K}=\left\{\left(r_{i}=\frac{i}{K}-1, r_{j}=\frac{j}{K}-1\right), 1 \leq i, j \leq 2 K-1\right\} \subset S,
$$

resulting in

$$
[B x]_{i j}=\sum_{k, \ell=1}^{2 N-1} \phi_{k}\left(r_{i}\right) \phi_{\ell}\left(r_{j}\right) x_{k \ell}
$$

- We assume that the initial distribution of temperatures $\left[u\left(0 ; p_{i}, p_{j}\right)\right]_{i, j=1}^{2 N-1}$ satisfies $\|u\|_{2} \leq R$, for some given $R$, implying that $x$ resides in the ellitope, namely, the ellipsoid

$$
\mathcal{X}=\left\{\left\{x_{k \ell}\right\} \in \mathbb{R}^{(2 N-1) \times(2 N-1)}: \sum_{k, \ell}\left[\mathrm{e}^{\left[\omega_{k}^{2}+\omega_{\ell}^{2}\right] t_{0}} x_{k \ell}\right]^{2} \leq R^{2}\right\}
$$




$$
\begin{gathered}
u\left(t ; p_{i}, p_{j}\right)=\sum_{k, \ell} \mathrm{e}^{-\left[\omega_{k}^{2}+\omega_{\ell}^{2}\right]\left[t-t_{0}\right]} \phi_{k}\left(p_{i}\right) \phi_{\ell}\left(p_{j}\right) x_{k \ell} \\
{[A(x)]_{\nu}=\sum_{k, \ell=1}^{2 N-1} x_{k \ell} \mathrm{e}^{-\left[\omega_{k}^{2}+\omega_{\ell}^{2}\right]\left[t_{1}-t_{0}\right]} \phi_{k}\left(p_{i(\nu)}\right) \phi_{\ell}\left(p_{j(\nu)}\right) x_{k \ell}}
\end{gathered}
$$

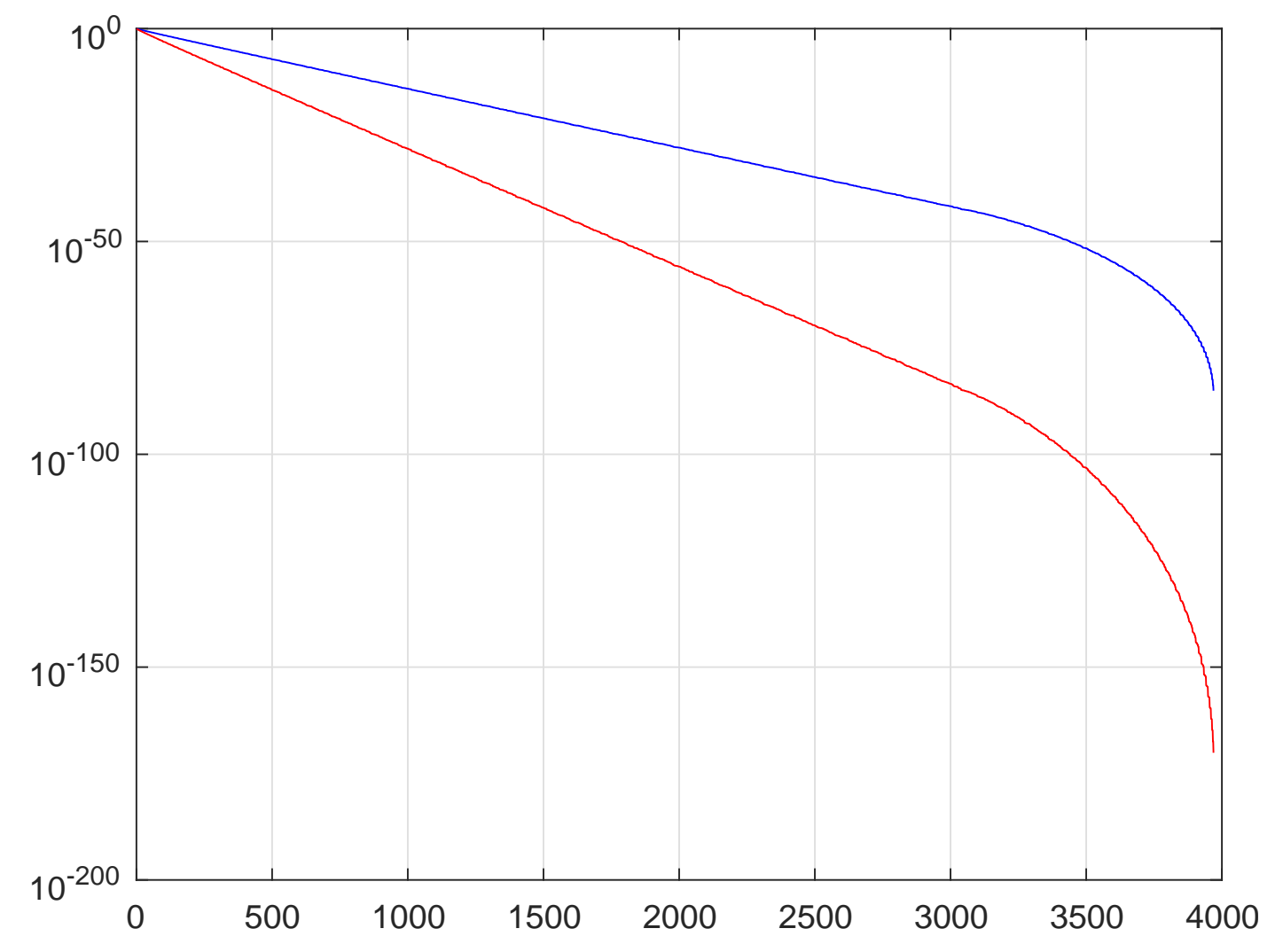

Plots of $\mathrm{e}^{-\left(\omega_{k}^{2}+\omega_{\ell}^{2}\right) t}$ for $t=0.02$ (red) and $t=0.01$ (blue) 
Bad news: contributions of high frequency (with large $\omega_{k}^{2}+\omega_{\ell}^{2}$ ) components $x_{k \ell}(0)$ to $A(x)$ decrease exponentially fast with high decay rate as $t_{!}$grows

$\Rightarrow$ High frequency components $x_{k \ell}(0)$ are impossible to recover from observations at time $t_{1}$, unless $t_{1}$ is very small (red curve, $t=0.02$ )

Good news: Contributions of high frequency components $x_{k \ell}(0)$ to $B(x)$ are very small, provided $t_{0}$ is not too small

$\Rightarrow$ There is no need to recover well high frequency components, provided they are not huge (blue curve, $t=0.01$ )

Numerical results $N=32, m=125, K=6, t_{0}=0.01, t_{1}=0.02$, $\sigma=0.001$,

$$
\mathcal{X}=\left\{x \in \mathbb{R}^{63 \times 63}:\left\|\left\{u\left(0 ; p_{i}, p_{j}\right)\right\}_{i, j=1}^{63}\right\|_{2} \leq 15\right\} .
$$

- Minimax risk of optimal linear estimate: 0.1707 


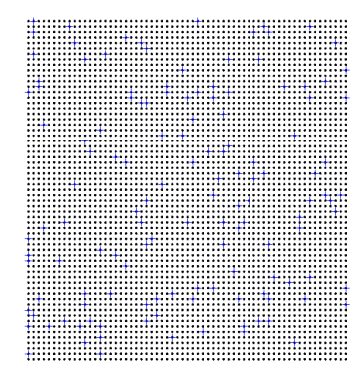

$63 \times 63$ grid $G_{63}$ and $m=125$ measurement points
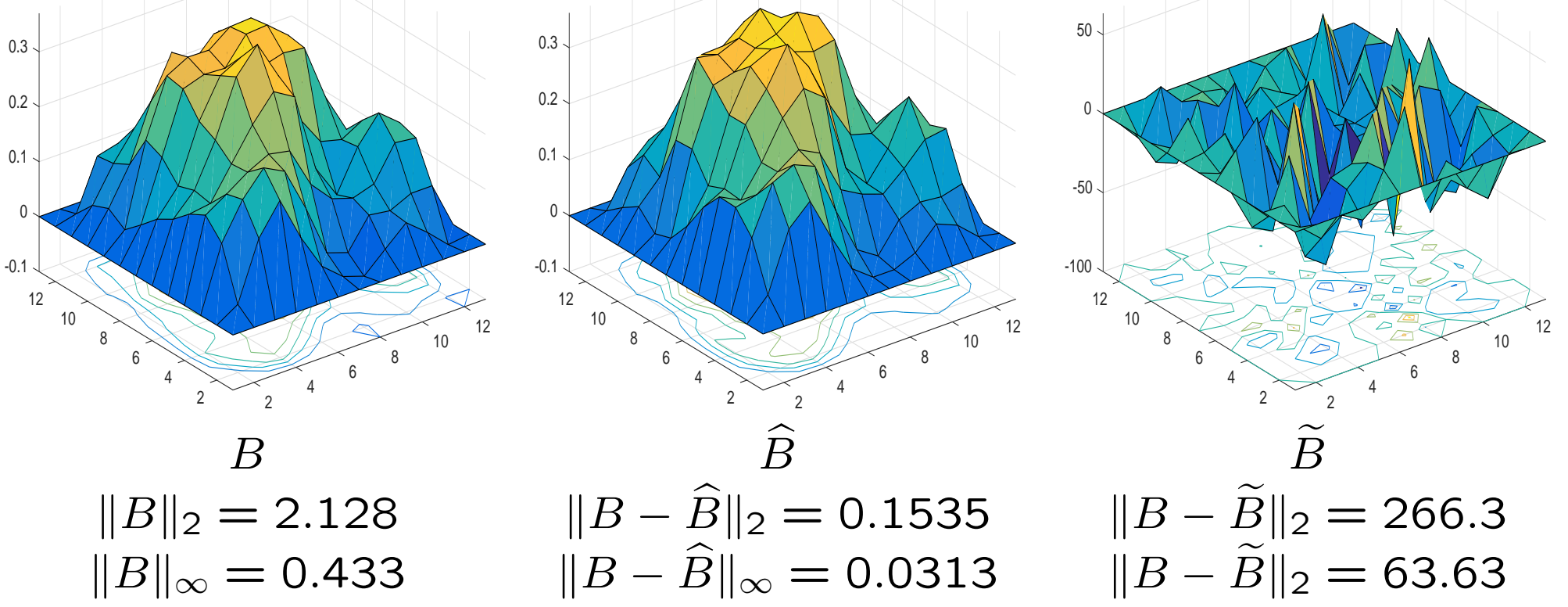

Left: true signal, center: linear recovery, right: naive LS recovery 


\section{Near-optimality of linear estimates}

$$
\mathcal{X}=\left\{x \in \mathbb{R}^{n}: \exists y \in \mathbb{R}^{N}, t \in \mathcal{T}: x=F y, y^{T} S_{k} y \leq t_{k} \forall k \leq K\right\}
$$

Simple observation: When recovering $B x, x \in \mathcal{X}$, from observation $\omega=$ $A x+\sigma \xi$, we lose nothing when assuming that the signal is

$$
y \in \mathcal{Y}=\left\{y: \exists t \in \mathcal{T}: y^{T} S_{k} y \leq t_{k} \forall k \leq K\right\}
$$

the observation is $\omega=[A F] y+\sigma \xi$, and the entity to be recovered is $[B F] y$. With this transformation, families of all estimates, all linear estimates and their risks remain intact

$\Rightarrow$ We lose nothing when assuming that $F$ is the identity, and

$$
\mathcal{X}=\left\{x \in \mathbb{R}^{n}: \exists t \in \mathcal{T}: x^{T} S_{k} x \leq t_{k} \forall k \leq K\right\}
$$




$$
\begin{aligned}
& \mathcal{X}=\left\{x \in \mathbb{R}^{n}: \exists t \in \mathcal{T}: x^{T} S_{k} x \leq t_{k} \forall k \leq K\right\} \Rightarrow \text { We can build linear estimate satisfying } \\
& \operatorname{Risk}^{2}[\widehat{x} \mid \mathcal{X}] \leq \mathrm{Opt}=\min _{H, \lambda}\left\{\phi_{\mathcal{T}}(\lambda)+\sigma^{2} \operatorname{Tr}\left(H^{T} H\right): \lambda \geq 0,\left[\begin{array}{c|c}
\sum_{k} \lambda_{k} S_{k} & B^{T}-A^{T} H \\
\hline B-H^{T} A & I_{\nu}
\end{array}\right] 0\right\}
\end{aligned}
$$

Our course of actions is as follows.

A. Suppose that we can specify Gaussian prior $\mathcal{N}(0, Q)$ for the signal $x$ to be supported on $\mathcal{X}$. Then by Gauss-Markov Theorem the quantity

$$
\psi(Q)=\operatorname{Tr}\left(B Q B^{T}-B Q A^{T}\left[\sigma^{2} I_{m}+A Q A^{T}\right]^{-1} A Q B^{T}\right)
$$

would be a lower bound on RiskOpt ${ }^{2}$.

B. While $\mathcal{N}(0, Q)$ cannot be supported on the bounded set $\mathcal{X}$ for a nonzero $Q$, we can select $Q$ to enforce $\eta \sim \mathcal{N}(0, Q)$ to "sit in $\mathcal{X}$ with overwhelming probability," so that a "slightly reduced" $\psi(Q)$ will become a lower bound on RiskOpt ${ }^{2}$. 
C. We have $\mathbf{E}_{\eta \sim \mathcal{N}(0, Q)}\left\{\eta^{T} S \eta\right\}=\operatorname{Tr}(S Q)$

$\Rightarrow$ Selecting $Q \succeq 0$ according to

$$
\exists t \in \mathcal{T}: \operatorname{Tr}\left(Q S_{k}\right) \leq t_{k}, k \leq K
$$

we ensure that $\eta \sim \mathcal{N}(0, Q)$ "sits in $\mathcal{X}$ on average"

$\Rightarrow$ We may expect that imposing on $Q \succeq 0$ the restriction

$$
\exists t \in \mathcal{T}: \operatorname{Tr}\left(Q S_{k}\right) \leq \rho t_{k}, k \leq K,
$$

we enforce $\eta \sim \mathcal{N}(0, Q)$ to take values in $\mathcal{X}$ with probability controlled by $\rho$ and approaching 1 as $\rho \rightarrow+0$. 


$$
\begin{aligned}
& \mathcal{X}=\left\{x \in \mathbb{R}^{n}: \exists t \in \mathcal{T}: x^{T} S_{k} x \leq t_{k} \forall k \leq K\right\} \Rightarrow \text { We can build linear estimate satisfying } \\
& \operatorname{Risk}^{2}[\widehat{x} \mid \mathcal{X}] \leq \mathrm{Opt}=\min _{H, \lambda}\left\{\phi_{\mathcal{T}}(\lambda)+\sigma^{2} \operatorname{Tr}\left(H^{T} H\right): \lambda \geq 0,\left[\begin{array}{c|c}
\sum_{k} \lambda_{k} S_{k} & B^{T}-A^{T} H \\
\hline B-H^{T} A & I_{\nu}
\end{array} \succeq 0\right\}\right.
\end{aligned}
$$

D. The above considerations give rise to parametric optimization problem

$$
\operatorname{Opt}_{*}(\rho)=\max _{Q \succeq 0}\left\{\psi(Q): \exists t \in \mathcal{T}: \operatorname{Tr}\left(Q S_{k}\right) \leq \rho t_{k} \forall k \leq K\right\}
$$

We can expect that for small $\rho$ "slightly corrected" $\operatorname{Opt}_{*}(\rho)$ is a lower bound on RiskOpt ${ }^{2}$.

Fact: By Conic Duality, $\operatorname{Opt}_{*}(\rho) \geq \rho \mathrm{Opt}$

$\Rightarrow$ Lower bounds on RiskOpt ${ }^{2}$ can be expressed via Opt, resulting in

$$
\sqrt{\mathrm{Opt}} \leq O(\sqrt{\ln (1 / \mathrm{RiskOpt})} \text { RiskOpt })
$$

provided RiskOpt is small. 


\section{From $\left(*_{1}\right)$ to its dual}

$$
\begin{array}{r}
\operatorname{Opt}_{*}(1)=\max _{Q, t}\left\{\operatorname{Tr}\left(B Q B^{T}-B Q A^{T}\left[\sigma^{2} I_{m}+A Q A^{T}\right]^{-1} A Q B^{T}\right):\right. \\
\left.t \in \mathcal{T}, Q \succeq 0, \operatorname{Tr}\left(Q S_{k}\right) \leq t_{k} \forall k\right\}
\end{array}
$$

Step 1: Passing to linear objective

$$
\begin{aligned}
\overbrace{\max _{Q, t}\left\{\operatorname{Tr}\left(B Q B^{T}-B Q A^{T}\left[\sigma^{2} I_{m}+A Q A^{T}\right]^{-1} A Q B^{T}\right): \begin{array}{l}
t \in \mathcal{T}, Q \succeq 0, \\
\operatorname{Tr}\left(Q S_{k}\right) \leq t_{k} \forall k
\end{array}\right\}}^{\operatorname{Opt}_{(1)}} \\
=\max _{Q, t, G}\left\{\operatorname{Tr}\left(B Q B^{T}\right)-\operatorname{Tr}(G): \begin{array}{l}
G-B Q A^{T}\left[\sigma^{2} I_{m}+A Q A^{T}\right]^{-1} A Q B^{T} \succeq 0 \\
t \in \mathcal{T}, Q \succeq 0, \operatorname{Tr}\left(Q S_{k}\right) \leq t_{k} \forall k
\end{array}\right\}
\end{aligned}
$$

[Schur Complement Lemma]

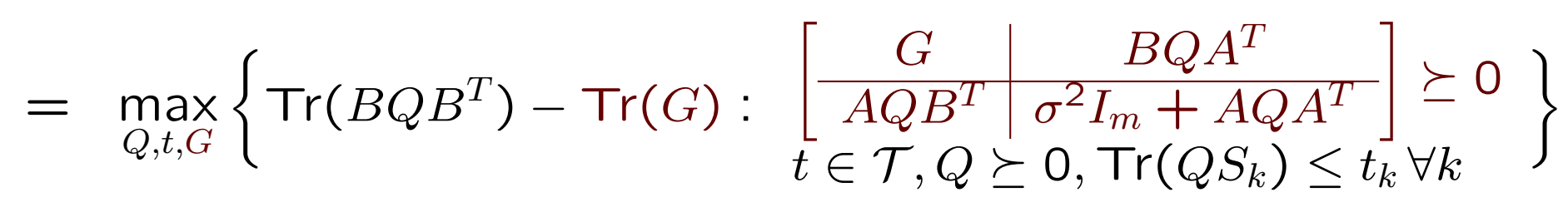

Step 2: Using the conic representation of $\mathcal{T}$ we get

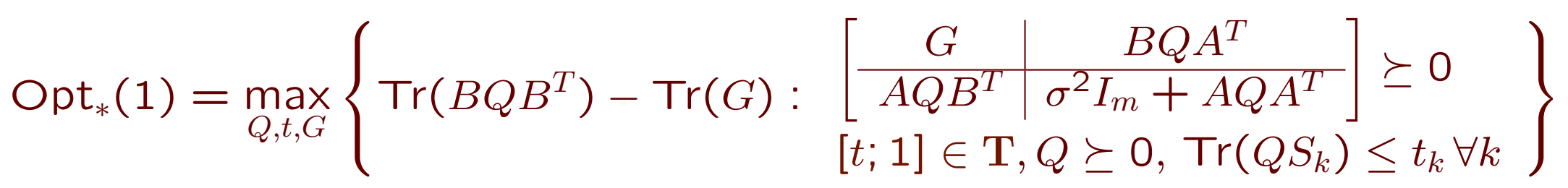

Note: $(*)$ is clearly strictly feasible and solvable. 


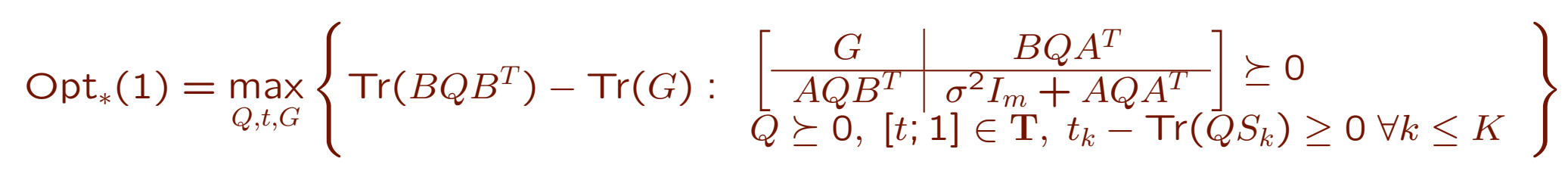

Step 3: passing from $(*)$ to the dual problem. We start with associating Lagrange multiplyers to the constraints (recall what $\mathbf{T}_{*}$ is):

$$
\underbrace{\left[\begin{array}{c|c}
G & B Q A^{T} \\
\hline A Q B^{T} & \sigma^{2} I_{m}+A Q A^{T}
\end{array}\right]}_{\left[\begin{array}{c|c}
U & V^{T} \\
\hline V & W
\end{array}\right] \succeq 0} \succeq 0, \underbrace{[t ; 1] \in \mathbf{T}}_{[g ; s] \in \mathbf{T}_{*}}, \underbrace{Q \succeq 0}_{P \succeq 0}, \underbrace{t_{k}-\operatorname{Tr}\left(Q S_{k}\right) \geq 0}_{\lambda_{k} \geq 0} \forall k \leq K
$$

- Taking inner products of the constraints of $(*)$ with Lagrange multipliers and summing up the results, we get the consequence of the constraints of $(*)$ :

$$
\begin{gathered}
\operatorname{Tr}(U G)+2 \operatorname{Tr}\left(V^{T} A Q B^{T}\right)+\operatorname{Tr}\left(W\left[\sigma^{2} I+A Q A^{T}\right]\right) \\
+g^{T} t+s+\operatorname{Tr}(P Q)+\sum_{k} \lambda_{k}\left[t_{k}-\operatorname{Tr}\left(Q S_{k}\right)\right] \geq 0 \\
\mathbb{1} \\
-\operatorname{Tr}(U G)+\operatorname{Tr}\left(\left[\sum_{k} \lambda_{k} S_{k}-P-A^{T} W A-B^{T} V^{T} A-A^{T} V B\right] Q\right)-[g+\lambda]^{T} t \leq s+\sigma^{2} \operatorname{Tr}(W) \\
{\left[\left[\begin{array}{l|l}
U & V^{T} \\
\hline V & W
\end{array}\right] \succeq 0, s \geq \phi_{\mathcal{T}}(-g), P \succeq 0, \lambda \geq 0\right]} \\
-4.55-
\end{gathered}
$$




$$
\begin{gathered}
-\operatorname{Tr}(U G)+\operatorname{Tr}\left(\left[\sum_{k} \lambda_{k} S_{k}-P-A^{T} W A-B^{T} V^{T} A-A^{T} V B\right] Q\right)-[g+\lambda]^{T} t \leq s+\sigma^{2} \operatorname{Tr}(W) \\
{\left[\left[\begin{array}{l|l}
U & V^{T} \\
\hline V & W
\end{array}\right] \succeq 0, s \geq \phi_{\mathcal{T}}(-g), P \succeq 0, \lambda \geq 0\right]}
\end{gathered}
$$

- To get the dual problem, we impose on Lagrange multiplies the requirement that the body of the aggregated - the red-constraint, as a function of $G, Q, t$, is identically equal to the objective

$$
\operatorname{Tr}\left(B Q B^{T}\right)-\operatorname{Tr}(G)
$$

of $(*)$, and minimize under this restriction (and the red constraints on the multipliers) the right hand side of the aggregated constraint.

By Conic Duality Theorem, we get

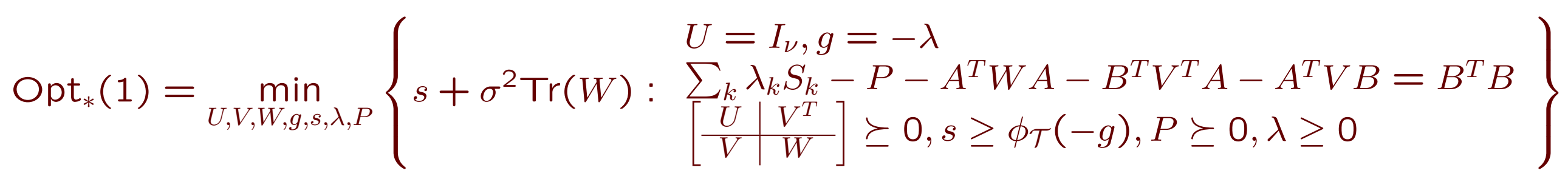




$$
\begin{aligned}
& \operatorname{Opt}_{*}(1)=\min _{U, V, W, g, s, \lambda, P}\left\{s+\sigma^{2} \operatorname{Tr}(W): \begin{array}{l}
U=I_{\nu}, g=-\lambda \\
\sum_{k} \lambda_{k} S_{k}-P-A^{T} W A-B^{T} V^{T} A-A^{T} V B=B^{T} B \\
{\left[\begin{array}{l|l}
U & V^{T} \\
\hline V & W
\end{array}\right] 0, s \geq \phi_{\mathcal{T}}(-g), P \succeq 0, \lambda \geq 0}
\end{array}\right\}
\end{aligned}
$$

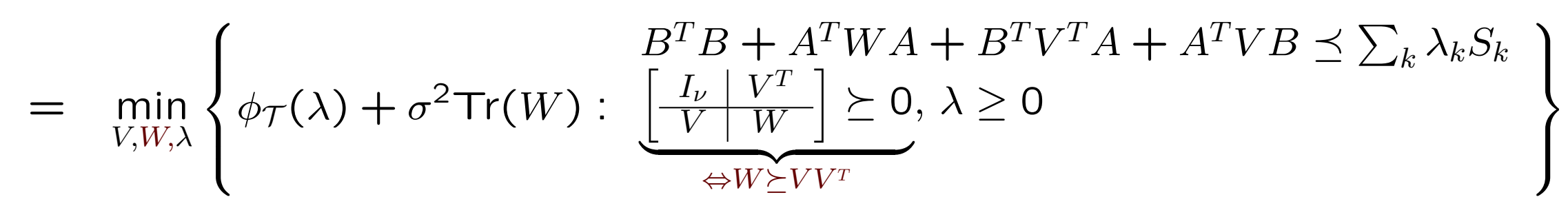

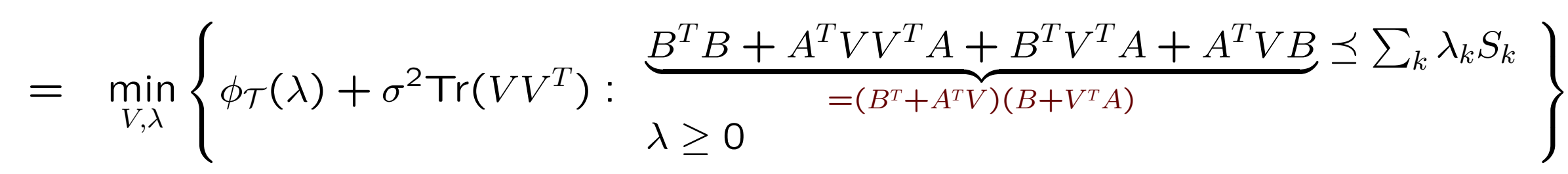

$$
\begin{aligned}
& \text { by Schur complement lemma } \\
& =\min _{V, \lambda}\left\{\phi_{\mathcal{T}}(\lambda)+\sigma^{2} \operatorname{Tr}\left(V V^{T}\right):\left[\begin{array}{c|c}
\sum_{k} \lambda_{k} S_{k} & B^{T}+A^{T} V \\
\hline B+V^{T} A & I_{\nu}
\end{array}\right] 0, \lambda \geq 0\right\} \\
& =\min _{H[:=-V], \lambda}\left\{\phi_{\mathcal{T}}(\lambda)+\sigma^{2} \operatorname{Tr}\left(H^{T} H\right):\left[\begin{array}{c|c}
\sum_{k} \lambda_{k} S_{k} & B^{T}-A^{T} H \\
\hline B-H^{T} A & I_{\nu}
\end{array}\right] \succeq 0, \lambda \geq 0\right\} \\
& =\text { : Opt }
\end{aligned}
$$


$\mathcal{X}=\left\{x \in \mathbb{R}^{n}: \exists t \in \mathcal{T}: x^{T} S_{k} x \leq t_{k} \forall k \leq K\right\} \Rightarrow$ We can build linear estimate satisfying $\operatorname{Risk}^{2}[\widehat{x} \mid \mathcal{X}] \leq \mathrm{Opt}=\min _{H, \lambda}\left\{\phi_{\mathcal{T}}(\lambda)+\sigma^{2} \operatorname{Tr}\left(H^{T} H\right): \lambda \geq 0,\left[\begin{array}{c|c}\sum_{k} \lambda_{k} S_{k} & B^{T}-A^{T} H \\ \hline B-H^{T} A & I_{\nu}\end{array}\right] 0\right\}=\mathrm{Opt}_{*}$

$$
\begin{gathered}
\mathrm{Opt}_{*}(\rho)=\max _{Q, t}\left\{\psi(Q): Q \succeq 0, t \in \mathcal{T}, \operatorname{Tr}\left(Q S_{k}\right) \leq \rho t_{k} \forall k\right\} \quad\left(*_{\rho}\right) \\
{[\sqrt{\psi(Q)}: \text { optimal Bayesian risk of recovering } B x \text { when } x \sim \mathcal{N}(0, Q)]}
\end{gathered}
$$

Easy fact: When $0<\rho \leq 1$, one has

$$
\operatorname{Opt}_{*}(\rho) \geq \rho \mathrm{Opt}_{*}(1)=\rho \mathrm{Opt} .
$$

Indeed, recall that

$$
\psi(Q)=\operatorname{Tr}\left(B Q B^{T}-B Q A^{T}\left[\sigma^{2} I_{m}+A Q A^{T}\right]^{-1} A Q B^{T}\right) .
$$

It follows immediately that $\psi(\rho Q) \geq \rho \psi(Q), 0 \leq \rho \leq 1$. 
Fact: If $Q_{\rho}$ stems from optimal solution to $(* \rho)$, then the probability for $\xi \sim \mathcal{N}\left(0, Q_{\rho}\right)$ to be outside of $\mathcal{X}$ approaches zero fast as $\rho$ decreases:

$$
\begin{gathered}
\pi(\rho):=\operatorname{Prob}_{\xi \sim \mathcal{N}\left(0, Q_{\rho}\right)}\{\xi \notin \mathcal{X}\} \leq K \exp \left\{-\frac{1-\rho+\rho \ln (\rho)}{2 \rho}\right\} \\
\begin{array}{||c||c|c|c|c|c|c||}
\hline \rho & 0.10 & 0.05 & 0.04 & 0.03 & 0.02 & 0.01 \\
\hline \pi(\rho) \leq & 1.0 \mathrm{e} 0 & 3.4 \mathrm{e}-2 & 3.1 \mathrm{e}-3 & 5.5 \mathrm{e}-5 & 1.6 \mathrm{e}-8 & 3.2 e-18 \\
K & K=100
\end{array}
\end{gathered}
$$

$\Rightarrow$ For reasonably small $\rho$, everything is as if $\mathcal{N}\left(0, Q_{\rho}\right)$ were supported on $\mathcal{X}$, so that, say, $0.99 \mathrm{Opt}_{*}(\rho) \geq 0.99 \rho \mathrm{Opt}$ is a lower bound on RiskOpt ${ }^{2}$. 
Theorem Let us associate with ellitope

$$
\mathcal{X}=\left\{x \in \mathbb{R}^{n}: \exists t \in \mathcal{T}: x^{T} S_{k} x \leq t_{k} \forall k \leq K\right\}
$$

the convex compact set

$$
\mathcal{Q}=\left\{Q \in \mathbf{S}^{n}: Q \succeq 0, \exists t \in \mathcal{T}: \operatorname{Tr}\left(Q S_{k}\right) \leq t_{k}, k \leq K\right\},
$$

and the quantity

$$
M_{*}=\max _{Q \in \mathcal{Q}} \sqrt{\operatorname{Tr}\left(B Q B^{T}\right)} .
$$

Then the linear estimate $\widehat{x}_{H_{*}}(\omega)=H_{*}^{T} \omega$ of $B x, x \in \mathcal{X}$, via observation $\omega=A x+\sigma \xi$, $\xi \sim \mathcal{N}\left(0, I_{m}\right)$, given by the optimal solution to the convex optimization problem

$$
\text { Opt }=\min _{H, \lambda}\left\{\phi_{\mathcal{T}}(\lambda)+\sigma^{2} \operatorname{Tr}\left(H^{T} H\right):\left[\begin{array}{c|c}
\sum_{k} \lambda_{k} S_{k} & B^{T}-A^{T} H \\
\hline B-H^{T} A & I_{\nu}
\end{array}\right] \succeq 0, \lambda \geq 0\right\}
$$

satisfies the risk bound

$$
\operatorname{Risk}\left[\widehat{x}_{H_{*}} \mid \mathcal{X}\right] \leq \sqrt{\mathrm{Opt}} \leq 4 \sqrt{\ln \left(\frac{6 M_{*}^{2} \sqrt{K}}{\operatorname{Risk}_{\text {Opt }}^{2}[\mathcal{X}]}\right)} \operatorname{Risk}_{\text {Opt }}[\mathcal{X}],
$$

where

$$
\text { Risk }_{\text {Opt }}[\mathcal{X}]=\inf _{\widehat{x}(\cdot)} \sup _{x \in \mathcal{X}} \sqrt{\mathbf{E}_{\xi \sim \mathcal{N}\left(0, I_{m}\right)}\left\{\|B x-\widehat{x}(A x+\sigma \xi)\|_{2}^{2}\right\}}
$$

inf being taken with respect to all, linear and nonlinear alike, estimates $\widehat{x}(\cdot)$, is the optimal minimax risk. 


\section{How it works: numerical illustration}

In these experiments

- $B$ is $n \times n$ identity matrix,

- $n \times n$ sensing matrix $A$ is a randomly rotated matrix with singular values $\lambda_{j}$, $1 \leq j \leq n$, forming a geometric progression, with $\lambda_{1}=1$ and $\lambda_{n}=0.01$.

- In the first experiment the signal set $\mathcal{X}_{1}$ is an ellipsoid:

$$
\mathcal{X}_{1}=\left\{x \in \mathbb{R}^{n}: \sum_{j=1}^{n} j^{2} x_{j}^{2} \leq 1\right\},
$$

that is, $K=1, S_{1}=\sum_{j=1}^{n} j^{2} e_{j} e_{j}^{T}$ ( $e_{j}$ are basic orths), and $\mathcal{T}=[0,1]$. Theoretical "suboptimality factor" in the interval $[31.6,73.7]$ in this experiment.

- In the second experiment, the signal set $\mathcal{X}$ is the box:

$$
\begin{aligned}
\mathcal{X}= & \left\{x \in \mathbb{R}^{n}: j\left|x_{j}\right| \leq 1,1 \leq j \leq n\right\} \\
& {\left[K=n, S_{k}=k^{2} e_{k} e_{k}^{T}, k=1, \ldots, K, \mathcal{T}=[0,1]^{K}\right] . }
\end{aligned}
$$

Theoretical "suboptimality factor" in the interval [73.2, 115.4]. 

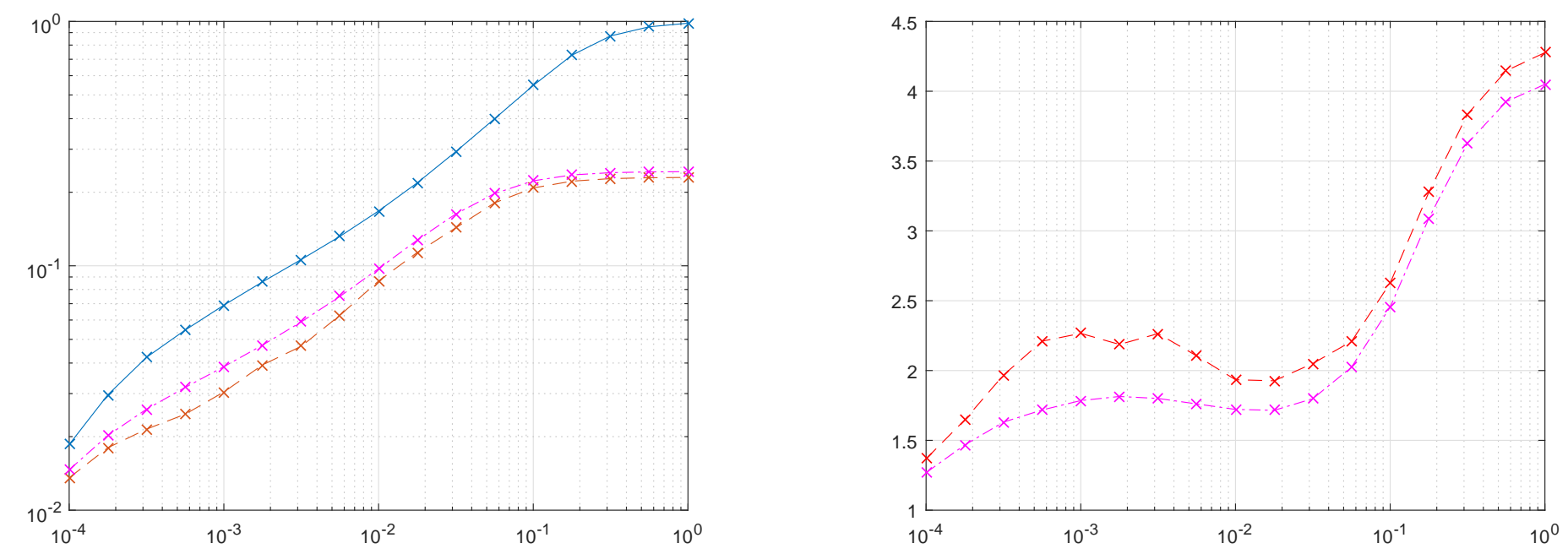

Recovery on ellipsoids: risk bounds as functions of the noise level $\sigma$, dimension $n=32$.

Left plot: upper and lower bounds of the risk; right plot: suboptimality ratios.
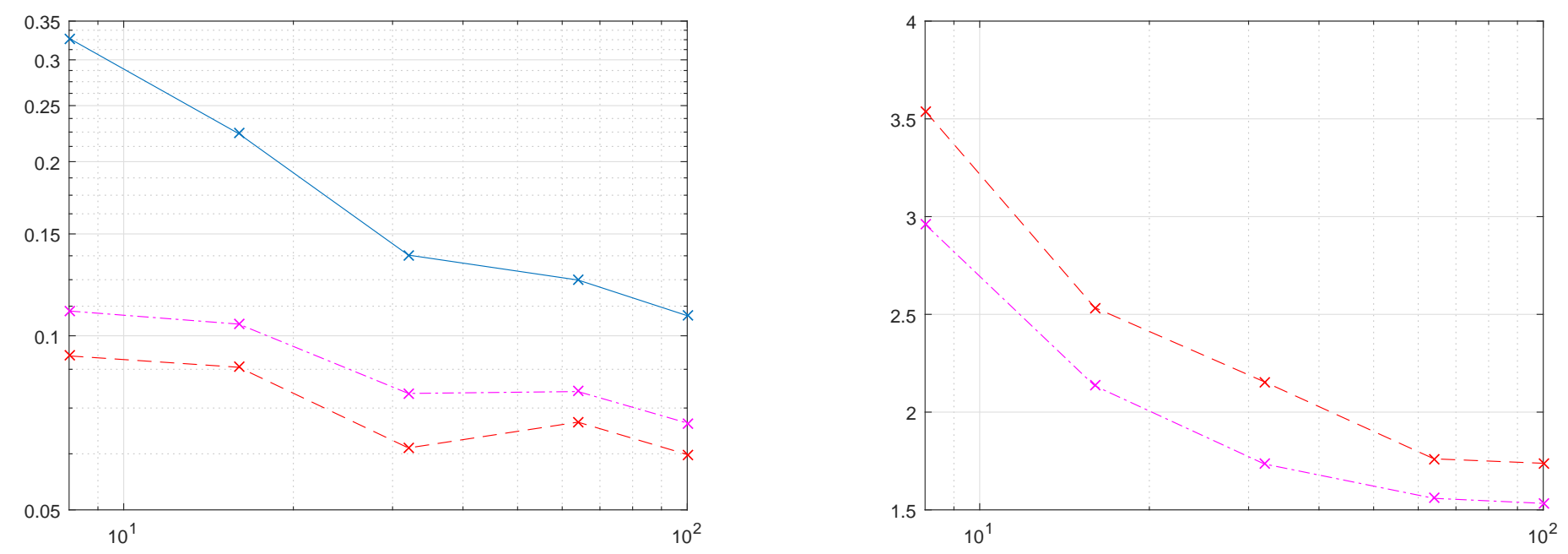

Recovery on ellipsoids: risk bounds as functions of problem dimension $n$, noise level $\sigma=0.01$. Left plot: upper and lower risk bounds; right plot: suboptimality ratios. 

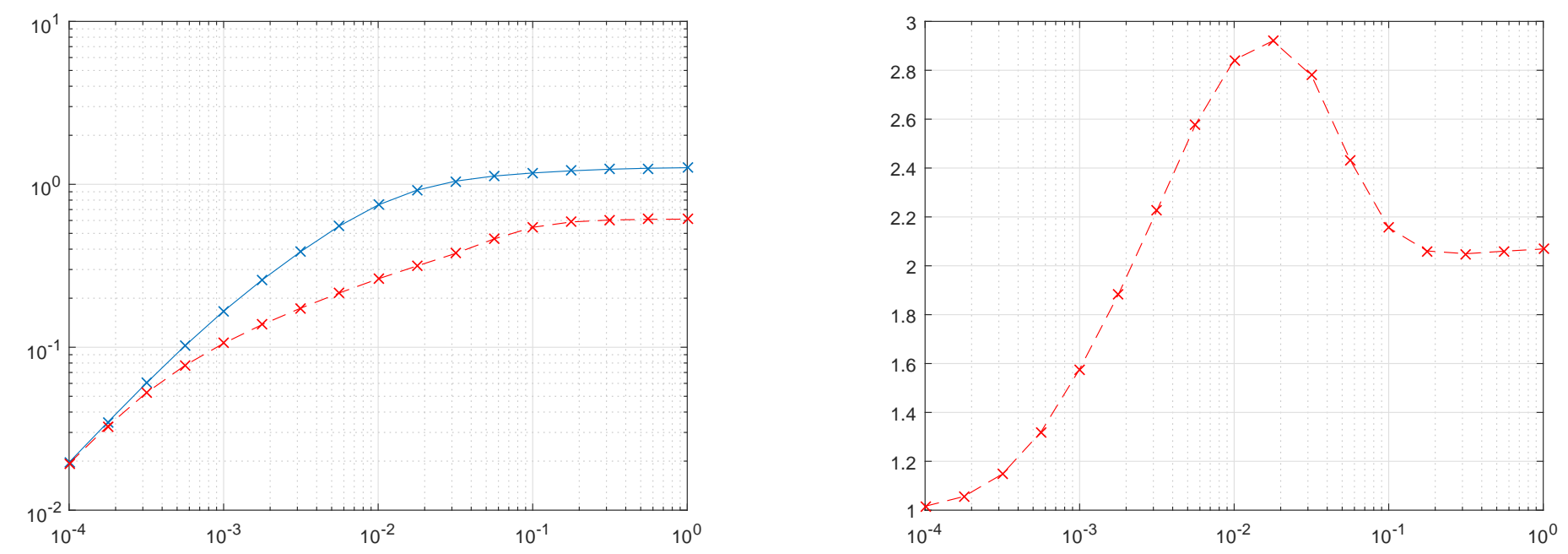

Recovery on a box: risk bounds as functions of the noise level $\sigma$, dimension $n=32$.

Left plot: upper and lower bounds of the risk; right plot: suboptimality ratios.
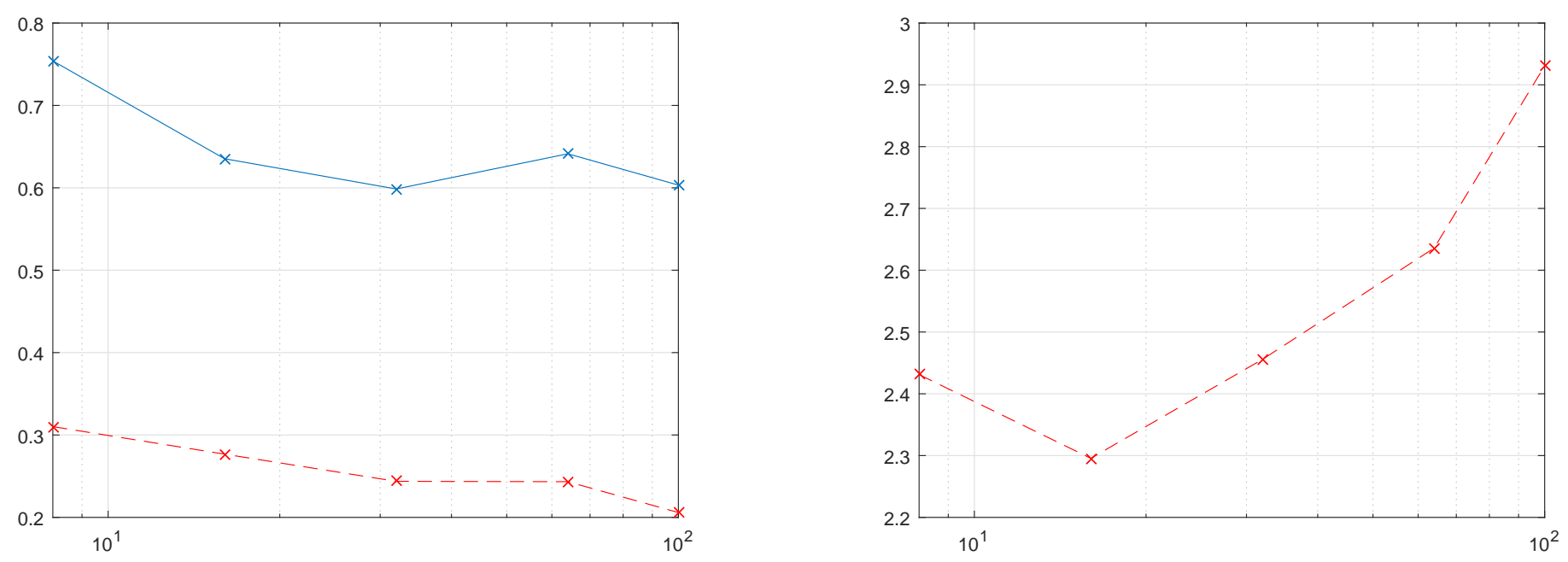

Recovery on a box: risk bounds as functions of problem dimension $n$, noise level $\sigma=0.01$. Left plot: upper and lower risk bounds; right plot: suboptimality ratios. 


\section{Extension: Relative Risks}

When "very large" signals are allowed, it might make sense to switch from the usual risk to its relative version - " $S$-risk" defined as follows:

$$
\operatorname{RiskS}[\widehat{x} \mid \mathcal{X}]=\min \left\{\sqrt{\tau}: \mathbf{E}_{\xi}\left\{\|B x-\widehat{x}(A x+\sigma \xi)\|_{2}^{2}\right\} \leq \tau\left[1+x^{T} S x\right] \forall x \in \mathcal{X}\right\}
$$

Note: Setting $S=0$ recovers the usual "plain" risk.

Results on design of near-optimal, in terms of plain risk, linear estimates extend to the case of $S$-risk. 
Design of near optimal linear estimate $\widehat{x}_{H_{*}}(\omega)=H_{*}^{T} \omega$ is given by an optimal solution $\left(H_{*}, \tau_{*}, \lambda_{*}\right)$ to the convex optimization problem

$$
\text { Opt }=\min _{H, \tau, \lambda}\left\{\begin{array}{c|c}
\tau: & {\left[\begin{array}{c|c}
\sum_{k} \lambda_{k} S_{k}+\tau S & B^{T}-A^{T} H \\
B-H^{T} A & I_{\nu}
\end{array}\right] \succeq 0,} \\
\sigma^{2} \operatorname{Tr}\left(H^{T} H\right)+\phi_{\mathcal{T}}(\lambda) \leq \tau, \lambda \geq 0
\end{array}\right\}
$$

For the resulting estimate, it holds $\operatorname{RiskS}\left[\widehat{x}_{H_{*}} \mid \mathcal{X}\right] \leq \sqrt{\mathrm{Opt}}$.

Near-optimality properties of the estimate $\widehat{x}_{H_{*}}$ remain the same as in the case of plain risk:

When $\xi \sim \mathcal{N}\left(0, I_{m}\right)$, one has

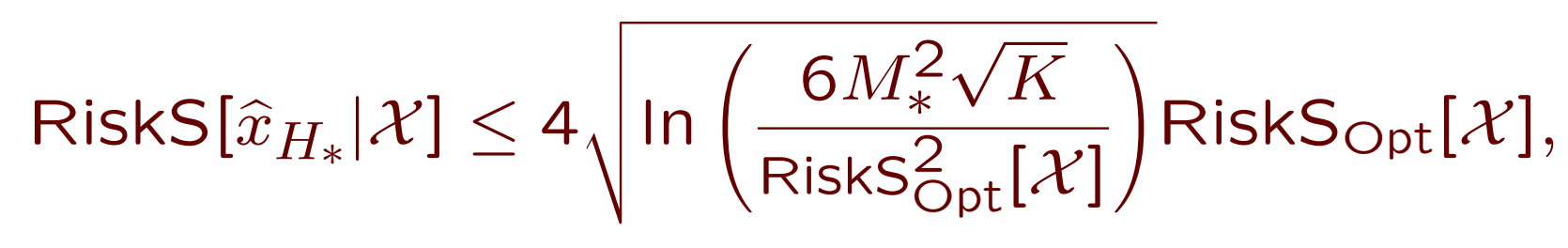

where

$$
M_{*}=\max _{Q}\left\{\sqrt{\operatorname{Tr}\left(B Q B^{T}\right)}: Q \succeq 0, \exists t \in \mathcal{T}: \operatorname{Tr}\left(Q S_{k}\right) \leq t_{k} \forall k \leq K\right\}
$$

and

$$
\operatorname{RiskS} \text { Opt }[\mathcal{X}]=\inf _{\widehat{x}(\cdot)} \operatorname{RiskS}[\widehat{x} \mid \mathcal{X}]
$$


In the special case $\mathcal{X}=\mathbb{R}^{n}$, the best linear estimate is yielded by optimal solution to the convex problem

$$
\text { Opt }=\min _{H, \tau}\left\{\tau:\left[\begin{array}{c|c}
\tau S & B^{T}-A^{T} H \\
\hline B-H^{T} A & I_{\nu}
\end{array}\right] \succeq 0, \sigma^{2} \operatorname{Tr}\left(H^{T} H\right) \leq \tau\right\}
$$

A feasible solution $\tau, H$ to $(*)$ gives rise to linear estimate $\widehat{x}_{H}(\omega)=H^{T} \omega$ such that

$$
\operatorname{RiskS}\left[\widehat{x}_{H} \mid \mathbb{R}^{n}\right] \leq \sqrt{\tau}
$$

provided $\xi$ is zero mean and with unit covariance matrix.

Proposition Assume that $B \neq 0$ and $(*)$ is feasible. Then the problem is solvable, and its optimal solution Opt, $H_{*}$ gives rise to linear estimate $\widehat{x}_{H_{*}}(\omega)=H_{*}^{T} \omega$ with $S$-risk $\sqrt{\mathrm{Opt}}$. When $\xi \sim \mathcal{N}\left(0, I_{m}\right)$, this estimate is minimax optimal:

$$
\operatorname{RiskS}\left[\widehat{x}_{H_{*}} \mid \mathbb{R}^{n}\right]=\sqrt{\mathrm{Opt}}=\operatorname{RiskS_{\text {Opt}}}\left[\mathbb{R}^{n}\right] .
$$




\section{A Byproduct: accuracy of Semidefinite Relaxation}

Theorem Let $C$ be a symmetric $n \times n$ matrix and $\mathcal{X}$ be an ellitope:

$$
\mathcal{X}=\left\{x \in \mathbb{R}^{n}: \exists(t \in \mathcal{T}, y): x=F y, y^{T} S_{k} y \leq t_{k} \forall k \leq K\right\} .
$$

Then the efficiently computable quantity

$$
\begin{gathered}
\text { Opt }=\min _{\lambda}\left\{\phi_{\mathcal{T}}(\lambda): \lambda \geq 0, F^{T} C F \preceq \sum_{k} \lambda_{k} S_{k}\right\} \\
{\left[\phi_{\mathcal{T}}(\lambda)=\max _{t \in \mathcal{T}} \lambda^{T} t\right]}
\end{gathered}
$$

is an upper bound on

$$
\mathrm{Opt}_{*}=\max _{x \in \mathcal{X}} x^{T} C x
$$

The above bound is tight, namely,

$$
\mathrm{Opt}_{*} \leq \mathrm{Opt} \leq 4 \ln (5 K) \mathrm{Opt}_{*} .
$$

Note: $\mathrm{Opt}_{*}$ is difficult to compute within $4 \%$ accuracy when $\mathcal{X}$ is as simple as the unit box in $\mathbb{R}^{n}$. 
Let $\mathcal{X}$ be given by quadratic inequalities:

$$
\begin{gathered}
\mathcal{X}=\left\{x \in \mathbb{R}^{n}: \exists t \in \mathcal{T}: x^{T} S_{k} x \leq t_{k}, k \leq K\right\} \neq \emptyset \\
{[\mathcal{T}: \text { nonempty convex compact set }]}
\end{gathered}
$$

We have

$$
\mathrm{Opt}_{*}:=\max _{x \in \mathcal{X}} x^{T} C x \leq \underbrace{\min _{\lambda}\left\{\phi_{\mathcal{T}}(\lambda): \lambda \geq 0, C \preceq \sum_{k} \lambda_{k} S_{k}\right\}}_{=: \text {Opt }} \leq \Theta \mathrm{Opt}_{*}
$$

Question: What can be said about the tightness factor $\Theta$ ?

A. When $K=1$, assuming Slater condition: $\bar{x}^{T} S_{1} \bar{x}<t$ for some $\bar{x}$ and some $t \in \mathcal{T}$, one can set $\Theta=1$. [the celebrated $\mathcal{S}$-Lemma]

B. Assuming that $x^{T} S_{k} x=x_{k}^{2}, k \leq K=\operatorname{dim} x, \mathcal{T}=[0 ; 1]^{K}$, and $C$ is Laplacian of a graph (i.e., off-diagonal entries in $C$ are nonpositive and all row sums are zero), one can set $\Theta=1.1382 \ldots$ [MAXCUT Theorem of Goemans and Williamson, 1995]

Note: Laplacian of a graph always is $\succeq 0$ 
C. When $C \succeq 0$ and all matrices $S_{k}$ are diagonal, one can set

$$
\Theta=\frac{\pi}{2}=1.5708 \ldots
$$

[ $\frac{\pi}{2}$ Theorem, Nesterov, 1998]

D. Assuming $\mathcal{X}$ is an ellitope (i.e., $S_{k} \succeq 0, \sum_{k} S_{k} \succ 0$ and $\mathcal{T}$ contains a positive vector), one can set $\Theta=4 \ln (5 K)$

Note: In the case of $\mathbf{D}, \Theta$ indeed can be as large as $O(\ln (K))$ 
A byproduct of the theorem is the following useful fact:

Theorem [upper-bounding of operator norms] Let $\|\cdot\|_{x}$ be a norm on $\mathbb{R}^{N}$ such that the unit ball $\mathcal{X}$ of the norm is an ellitope:

$$
\mathcal{X}:=\left\{x:\|x\|_{x} \leq 1\right\}=\left\{x: \exists(t \in \mathcal{T}, y): x=P y, y^{T} S_{k} y \leq t_{k}, k \leq K\right\}
$$

Let, further, $\|\cdot\|$ be a norm on $\mathbb{R}^{M}$ such that the unit ball $\mathcal{B}^{*}$ of the norm $\|\cdot\|_{*}$ conjugate to $\|\cdot\|$ is an ellitope:

$$
\begin{aligned}
\mathcal{B}_{*} & :=\left\{u \in \mathbb{R}^{m}: u^{T} v \leq 1 \forall(v,\|v\| \leq 1)\right\} \\
& =\left\{u: \exists(r \in \mathcal{R}, z): u=Q z, z^{T} R_{\ell} z \leq r_{\ell}, \ell \leq L\right\}
\end{aligned}
$$

Then the efficiently computable quantity

$$
\begin{gathered}
\operatorname{Opt}(C)=\min _{\lambda, \mu}\left\{\phi_{\mathcal{T}}(\lambda)+\phi_{\mathcal{R}}(\mu): \lambda \geq 0, \mu \geq 0,\left[\begin{array}{c|c}
\sum_{\ell} \mu_{\ell} R_{\ell} & \frac{1}{2} Q^{T} C P \\
\hline \frac{1}{2} P^{T} C^{T} Q & \sum_{k} \lambda_{k} S_{k}
\end{array}\right] \succeq 0\right\} \\
{\left[C \in \mathbb{R}^{M \times N}\right]}
\end{gathered}
$$

is a convex in $C$ upper bound on the operator norm

$$
\|C\|_{\|\cdot\| x \rightarrow\|\cdot\|}=\max _{x}\left\{\|C x\|:\|x\|_{x} \leq 1\right\}
$$

of the mapping $x \mapsto C x$, and this bound is reasonably tight:

$$
\|C\|_{\|\cdot\|_{x} \rightarrow\|\cdot\|} \leq \operatorname{Opt}(C) \leq 4 \ln (5(K+L))\|C\|_{\|\cdot\|_{x} \rightarrow\|\cdot\|} .
$$


Indeed, the operator norm in question is the maximum of a quadratic form over an ellitope:

$$
\begin{aligned}
\|C\|_{\|\cdot\| x \rightarrow\|\cdot\|} & =\max _{x \in \mathcal{X}, u \in \mathcal{B}_{*}}[x ; u]^{T}\left[\left.\frac{1}{\frac{1}{2} C^{T}}\right|^{\frac{1}{2} C}\right][x ; u] \\
& =\max _{[y ; z] \in \mathcal{W}}[y ; z]^{T}\left[\left.\frac{1}{\frac{1}{2} P^{T} C^{T} Q}\right|^{\frac{1}{2} Q^{T} C P}\right][y ; z]
\end{aligned}
$$

where $\mathcal{W}$ is the basic ellitope given by

$$
\mathcal{W}=\left\{[y ; z]: \exists[t ; r] \in \mathcal{T} \times \mathcal{R}: y^{T} S_{k} y \leq t_{k}, k \leq K, z^{T} R_{\ell} z \leq r_{\ell}, \ell \leq L\right\} .
$$




\section{Inside Semidefinite Relaxation}

$$
\mathrm{Opt}_{*}=\max _{x, t}\left\{x^{T} C x: x^{T} S_{k} x \leq t_{k}, k \leq K, t \in \mathcal{T}\right\}
$$

Fact: Semidefinite relaxation admits an alternative description as follows:

Let us associate with $(*)$ another optimization problem where instead of deterministic candidate solutions $(x, t)$ we are looking for random solutions $(\xi, \tau)$ satisfying the constraints on average:

$$
\mathrm{Opt}^{+}=\max _{\xi, \tau}\left\{\mathbf{E}\left\{\xi^{T} C \xi\right\}: \begin{array}{l}
\mathbf{E}\left\{\xi^{T} S_{k} \xi\right\} \leq \mathbf{E}\left\{\tau_{k}\right\} \\
\mathbf{E}\{\tau\} \in \mathcal{T}
\end{array}\right\}
$$

Immediate observation: Property of a random solution $(\xi, \tau)$ to be feasible for (!) depends solely on the matrix $Q=\mathbf{E}\left\{\xi \xi^{T}\right\}$ and the vector $t=\mathbf{E}\{\tau\}$, so that

$$
\mathrm{Opt}^{+}=\max _{Q, t}\left\{\operatorname{Tr}(C Q): \begin{array}{l}
\operatorname{Tr}\left(S_{k} Q\right) \leq t_{k} \\
Q \succeq 0, t \in \mathcal{T}
\end{array}\right\}=\max _{Q, t}\left\{\operatorname{Tr}(C Q): \begin{array}{l}
\operatorname{Tr}\left(S_{k} Q\right) \leq t_{k} \\
Q \succeq 0,[t ; 1] \in \mathbf{T}
\end{array}\right\}
$$




$$
\begin{aligned}
\text { Opt }_{*} & =\max _{x, t}\left\{x^{T} C x: \exists(t \in \mathcal{T}): x^{T} S_{k} x \leq t_{k}\right\} \\
\text { Opt }^{+} & =\max _{Q, t}\left\{\operatorname{Tr}(C Q): \operatorname{Tr}\left(S_{k} Q\right) \leq t_{k}, Q \succeq 0,[t ; 1] \in \mathbf{T}\right\}
\end{aligned}
$$

Note: (\#) is strictly feasible and bounded, and the problem

$$
\text { Opt }=\min _{\lambda}\left\{\phi_{\mathcal{T}}(\lambda): \lambda \geq 0, C \preceq \sum_{k} \lambda_{k} S_{k}\right\}
$$

specifying Semidefinite Relaxation upper bound on Opt is nothing but the conic dual to (\#)

$$
\Rightarrow \mathrm{Opt}^{+}=\mathrm{Opt}
$$


(\#) suggests the following recipe for quantifying the conservatism of the upper bound Opt on Opt : $^{2}$

- Find an optimal solution $Q_{*}, t_{*}$ to (\#) and treat $Q_{*} \succeq 0$ as the matrix of second moments of random vector $\xi$ (many options!)

- Random solutions $\left(\xi, t_{*}\right)$ satisfy $(*)$ "at average." Try to "correct" them to get feasible solutions to $(*)$ and look how "costly" this correction is in terms of the objective.

For example, in Goemans-Williamson MAXCUT and in Nesterov's $\pi / 2$ theorems, where $x^{T} C x$ is maximized over the unit box $\left\{\|x\|_{\infty} \leq 1\right\}$, one selects $\xi \sim \mathcal{N}\left(0, Q_{*}\right)$ and "corrects" $\xi$ according to $\xi \mapsto \operatorname{sign}[\xi]$. 


$$
\begin{aligned}
\text { Opt }_{*} & =\max _{x, t}\left\{x^{T} C x: \exists(t \in \mathcal{T}): x^{T} S_{k} x \leq t_{k}\right\} \\
\text { Opt }^{+} & =\max _{Q, t}\left\{\operatorname{Tr}(C Q): \operatorname{Tr}\left(S_{k} Q\right) \leq t_{k}, Q \succeq 0,[t ; 1] \in \mathbf{T}\right\}
\end{aligned}
$$

This is how the above recipe works in the general ellitopic case:

A. Let $\left(Q_{*}, t^{*}\right)$ be an optimal solution to $(\#)$. Set

$$
\bar{C}:=Q_{*}^{1 / 2} C Q_{*}^{1 / 2}=U D U^{T}
$$

( $U$ is orthogonal, $D$ is diagonal).

B. Let $\xi=Q_{*}^{1 / 2} U \zeta$ with Rademacher r.v. $\zeta\left(\zeta_{i}\right.$ take values \pm 1 with probability $1 / 2$ and are independent across $i$ ), so that $\mathbf{E}\left\{\xi \xi^{T}\right\}=Q_{*}$. Note that

$$
\begin{aligned}
\xi^{T} C \xi & =\zeta^{T} U^{T}\left[Q_{*}^{1 / 2} C Q_{*}^{1 / 2}\right] U \zeta=\zeta^{T} D \zeta \equiv \operatorname{Tr}(D) \\
& =\operatorname{Tr}\left(Q_{*}^{1 / 2} C Q_{*}^{1 / 2}\right) \equiv \operatorname{Tr}\left(C Q_{*}\right)=\text { Opt } \\
\mathbf{E}\left\{\xi^{T} S_{k} \xi\right\} & =\mathbf{E}\left\{\zeta^{T} U^{T} Q_{*}^{1 / 2} S_{k} Q_{*}^{1 / 2} U \zeta\right\}=\operatorname{Tr}\left(U^{T} Q_{*}^{1 / 2} S_{k} Q_{*}^{1 / 2} U\right) \\
& =\operatorname{Tr}\left(Q_{*}^{1 / 2} S_{k} Q_{*}^{1 / 2}\right)=\operatorname{Tr}\left(S_{k} Q_{*}\right) \leq t_{k}^{*}, \quad k \leq K
\end{aligned}
$$




$$
\xi^{T} C \xi \equiv \text { Opt } \quad(a) \quad \mathrm{E}\left\{\xi^{T} S_{k} \xi\right\} \leq t_{k}^{*}, k \leq K
$$

C. Since $S_{k} \succeq 0$ and $\xi$ is "light-tail" (it comes from Rademacher random vector), simple bounds on probabilities of large deviations combine with (b) to imply that

$$
\forall(\gamma \geq 0, k \leq K): \operatorname{Prob}\left\{\xi: \xi^{T} S_{k} \xi>\gamma t_{k}^{*}\right\} \leq O(1) \exp \{-O(1) \gamma\}
$$

$\Rightarrow$ with $\gamma_{*}=O(1) \ln (K+1)$, there exists a realization $\widehat{\xi}$ of $\xi$ such that

$$
\widehat{\xi}^{T} S_{k} \widehat{\xi} \leq \gamma_{*} t_{k}^{*}, k \leq K
$$

$\Rightarrow\left(\xi^{*}=\frac{\widehat{\xi}}{\sqrt{\gamma_{*}}}, t^{*}\right)$ is feasible for

$$
\mathrm{Opt}_{*}=\max _{x, t}\left\{x^{T} C x: \exists(t \in \mathcal{T}): x^{T} S_{k} x \leq t_{k}\right\}
$$

$\Rightarrow \mathrm{Opt}_{*} \geq \gamma_{*}^{-1} \widehat{\xi}^{T} C \widehat{\xi}=\gamma_{*}^{-1}$ Opt (look at $\left.(a) !\right)$ 
A simple bounds on deviation probabilities stems from the following

Mini-Lemma Let $P$ be positive semidefinite $N \times N$ matrix with trace $\leq 1$ and $\zeta$ be $N$-dimensional Rademacher random vector. Then

$$
\mathrm{E}\left\{\exp \left\{\zeta^{T} P \zeta / 3\right\}\right\} \leq \sqrt{3} \Rightarrow \operatorname{Prob}\left\{\zeta^{T} P \zeta>\gamma\right\} \leq \sqrt{3} e^{-\gamma / 3}
$$

Mini-Lemma $\Rightarrow$ bounds: We have

$$
\xi^{T} S_{k} \xi=\zeta^{T} \underbrace{U^{T} Q_{*}^{1 / 2} S_{k} Q_{*}^{1 / 2} U}_{=: t_{k}^{*} P_{k}} \zeta
$$

with $\operatorname{Tr}\left(P_{k}\right)=\operatorname{Tr}\left(Q_{*}^{1 / 2} S_{k} Q_{*}^{1 / 2}\right) / t_{k}^{*}=\operatorname{Tr}\left(S_{k} Q_{*}\right) / t_{k}^{*} \leq 1$

$\Rightarrow[$ Mini-Lemma $] \operatorname{Prob}\left\{\zeta^{T} P_{k} \zeta>3 \rho\right\} \leq \sqrt{3} e^{-\rho}$

$\Rightarrow \operatorname{Prob}\left\{\xi^{T} S_{k} \xi>\gamma t_{k}^{*}\right\}=\operatorname{Prob}\left\{\zeta^{T} P_{k} \zeta>\gamma\right\} \leq \sqrt{3} e^{-\gamma / 3}$. 
Proof of the lemma: Let $P=\sum_{i} \sigma_{i} f_{i} f_{i}^{T}$ be the eigenvalue decomposition of $P$, so that $f_{i}^{T} f_{i}=1, \sigma_{i} \geq 0$, and $\sum_{i} \sigma_{i} \leq 1$. The function

$$
F\left(\sigma_{1}, \ldots, \sigma_{N}\right)=\mathbf{E}\left\{e^{\frac{1}{3} \sum_{i} \sigma_{i} \zeta^{T} f_{i} f_{i}^{T} \zeta}\right\}
$$

is convex on the simplex $\left\{\sigma \geq 0, \sum_{i} \sigma_{i} \leq 1\right\}$ and thus attains it maximum over the simplex at a vertex, implying that for some $h=f_{i}, h^{T} h=1$, it holds

$$
\mathbf{E}\left\{e^{\frac{1}{3} T^{T} P \zeta}\right\} \leq \mathbf{E}\left\{e^{\frac{1}{3}\left(h^{T} \zeta\right)^{2}}\right\}
$$

Let $\xi \sim \mathcal{N}(0,1)$ be independent of $\zeta$. We have

$$
\begin{aligned}
\mathbf{E}_{\zeta}\left\{e^{\frac{1}{3}\left(h^{T} \zeta\right)^{2}}\right\} & =\mathbf{E}_{\zeta}\left\{\mathbf{E}_{\xi}\left\{\exp \left\{\left[\sqrt{2 / 3} h^{T} \zeta\right] \xi\right\}\right\}\right\}=\mathbf{E}_{\xi}\left\{\mathbf{E}_{\zeta}\left\{\exp \left\{\left[\sqrt{2 / 3} h^{T} \zeta\right] \xi\right\}\right\}\right\} \\
& =\mathbf{E}_{\xi}\left\{\prod_{s=1}^{N} \mathbf{E}_{\zeta}\left\{\exp \left\{\sqrt{2 / 3} \xi h_{s} \zeta_{s}\right\}\right\}\right\} \\
& =\mathbf{E}_{\xi}\left\{\prod_{s=1}^{N} \cosh \left(\sqrt{2 / 3} \xi h_{s}\right)\right\} \leq \mathbf{E}_{\xi}\left\{\prod_{s=1}^{N} \exp \left\{\xi^{2} h_{s}^{2} / 3\right\}\right\} \\
& =\mathbf{E}_{\xi}\left\{\exp \left\{\xi^{2} / 3\right\}\right\}=\sqrt{3}
\end{aligned}
$$




\section{Extensions}

So far, we have considered a problem of recovering $B x$ from observation

$$
\omega=A x+\eta
$$

where

- $x$ is unknown signal known to belong to a given basic ellitope

$$
\mathcal{X}=\left\{x \in \mathbb{R}^{n}: \exists t \in \mathcal{T}: x^{T} S_{k} x \leq t_{k}, k \leq K\right\}
$$

- $A \in \mathbb{R}^{m \times n}$ and $B \in \mathbb{R}^{\nu \times n}$ are given matrices

- $\eta \sim \mathcal{N}\left(0, \sigma^{2} I_{m}\right)$ is observation noise item (squared) risk of a candidate estimate is the worst-case, over $x \in \mathcal{X}$, expected squared $\|\cdot\|_{2}$-norm of recovery error:

$$
\operatorname{Risk}^{2}[\widehat{x} \mid \mathcal{X}]=\sup _{x \in \mathcal{X}} \mathbf{E}\left\{\|B x-\widehat{x}(A x+\eta)\|_{2}^{2}\right\} .
$$


We are about to extend our results to the situation where

- Noise $\eta$ not necessary is zero mean Gaussian; we allow the distribution $P$ of noise to be unknown in advance and to depend on signal $x$. Assumption: The matrix of second moments of $P$ admits a known upper bound:

$$
\vee[P]:=\mathbf{E}_{\eta \sim P}\left\{\eta \eta^{T}\right\} \preceq Q
$$

for a given $Q \succ 0$

- We measure recovering error in a given norm $\|\cdot\|$, not necessarily the Euclidean one, and define risk of a candidate estimate $\widehat{x}(\cdot)$ as

$$
\text { Risk }_{Q,\|\cdot\|}[\widehat{x} \mid \mathcal{X}]=\sup _{x \in \mathcal{X} P: \operatorname{Var}[P] \preceq Q} \mathbf{E}_{\eta \sim P}\{\|B x-\widehat{x}(A x+\eta)\|\}
$$

Assumption: The unit ball $\mathcal{B}_{*}$ of the norm conjugate to $\|\cdot\|$ is an ellitope:

$$
\begin{gathered}
\|u\|=\max _{h \in \mathcal{B}^{*}} h^{T} u, \\
\mathcal{B}_{*}=\left\{h: \exists\left(y \in \mathbb{R}^{M}, r \in \mathcal{R}\right): h=F y, y^{T} R_{\ell} y \preceq r_{\ell} \forall \ell \leq L\right\}
\end{gathered}
$$




\section{Building linear estimate}

We have

$$
\begin{aligned}
& \operatorname{Risk}_{Q,\|\cdot\|}\left[\widehat{x}_{H} \mid \mathcal{X}\right]=\sup _{x \in \mathcal{X}} \sup _{P: \operatorname{Var}[P] \preceq Q} \mathbf{E}_{\eta \sim Q}\left\{\left\|B x-H^{T}[A x+\eta]\right\|\right\} \\
& \left.\leq \sup _{x \in \mathcal{X}} \sup _{P: \operatorname{Var}[P] \preceq Q} \mathbf{E}_{\eta \sim Q}\left\{\left\|\left[B-H^{T} A x\right]\right\|+\| H^{T} \eta\right] \|\right\} \\
& \leq \Phi(H)+\Psi_{Q}[H], \\
& {\left[\Phi(H)=\max _{x \in \mathcal{X}}\left\|\left[B-H^{T} A\right] x\right\|, \quad \Psi_{Q}(H)=\sup _{P: \operatorname{Var}[P] \preceq Q} \mathbf{E}_{\eta \sim P}\left\{\left\|H^{T} \eta\right\|\right\}\right]}
\end{aligned}
$$


Next,

$$
\mathcal{B}_{*}=\{u=M y: y \in \mathcal{Y}\}, \mathcal{Y}=\left\{y: \exists r \in \mathcal{R}: y^{T} R_{\ell} y \leq r_{\ell} \forall \ell \leq L\right\}
$$

whence

$$
\begin{aligned}
& \Phi(H):=\max _{x \in \mathcal{X}}\left\|\left[B-H^{T} A\right] x\right\|
\end{aligned}
$$

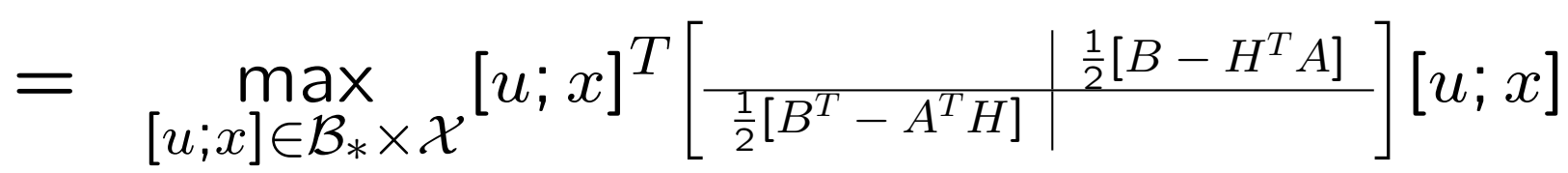

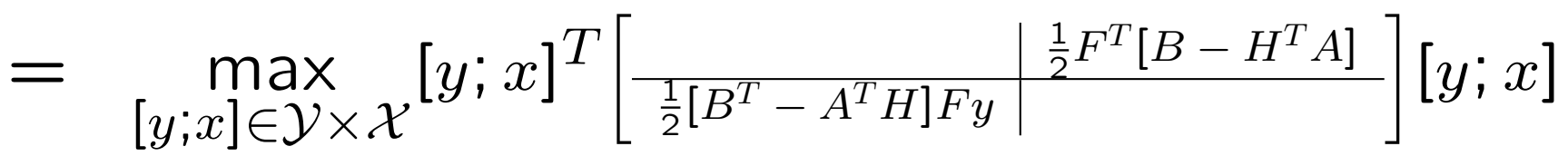

[semidefinite relaxation; note that $\mathcal{Y} \times \mathcal{X}$ is an ellitope]

Thus,

$$
\begin{aligned}
& \Phi(H) \leq \Phi(H):=\min _{\lambda, \mu}\left\{\begin{array}{l|l}
\left.\left.\phi_{\mathcal{T}}(\lambda)+\phi_{\mathcal{R}}(\mu): \begin{array}{c|c}
\sum_{\ell} \mu_{\ell} R_{\ell} & \frac{1}{2} F^{T}\left[H^{T} A-B\right] \\
\hdashline \frac{1}{2}\left[A^{T} H-B^{T}\right] F & \sum_{k} \lambda_{k} S_{k}
\end{array}\right] \succeq 0\right\} \\
\lambda \geq 0, \mu \geq 0
\end{array}\right\} \\
& {\left[\phi_{\mathcal{T}}(\lambda)=\max _{t \in \mathcal{T}} \lambda^{T} t, \phi_{\mathcal{R}}(\mu)=\max _{r \in \mathcal{R}} \mu^{T} r\right]}
\end{aligned}
$$




\section{Lemma One has}

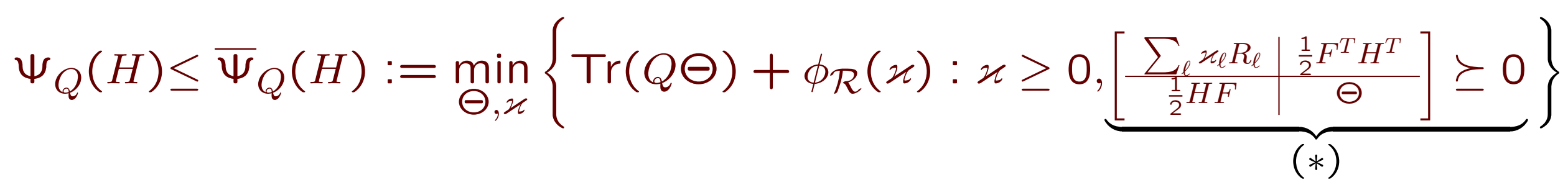

Indeed, let $(\varkappa, \Theta)$ be feasible for the right hand side problem, and let $\mathrm{V}[P] \preceq Q$

$$
\begin{aligned}
\left\|H^{T} \xi\right\| & =\max _{u \in \mathcal{B}_{*}}\left[-u^{T} H^{T} \xi\right]=\max _{y \in \mathcal{Y}}\left[-y^{T} F^{T} H^{T} \xi\right] \\
& \leq \max _{y \in \mathcal{Y}}\left[y^{T}\left[\sum_{\ell} \varkappa_{\ell} R_{\ell}\right] y+\xi^{T} \Theta \xi\right][\text { by }(*)] \\
& =\max _{r \in \mathcal{R}, y}\left\{y^{T}\left[\sum_{\ell} \varkappa_{\ell} R_{\ell}\right] y+\xi^{T} \Theta \xi: y^{T} R_{\ell} y \leq r_{\ell}, \ell \leq L\right\} \\
& \leq \max _{r \in \mathcal{R}}\left\{\sum_{\ell} \varkappa_{\ell} r_{\ell}+\xi^{T} \Theta \xi\right\} \\
& \leq \phi_{\mathcal{R}}(\varkappa)+\xi^{T} \Theta \xi=\phi_{\mathcal{R}}(\varkappa)+\operatorname{Tr}\left(\Theta\left[\xi \xi^{T}\right]\right) .
\end{aligned}
$$

Taking expectation in $\xi$, the conclusion of Lemma follows.

Illustration: When $\|\cdot\|=\|\cdot\|_{p}, p \in[1,2]$, Lemma states that whenever $\vee[P] \preceq Q$, one has

$$
\mathbf{E}_{\eta \sim P}\left\{\left\|H^{T} \eta\right\|_{p}\right\} \leq\left\|\left[\left\|\mathrm{Col}_{1}\left[Q^{1 / 2} H\right]\right\|_{2} ; \ldots ;\left\|\mathrm{Col}_{\nu}\left[Q^{1 / 2} H\right]\right\|_{2}\right]\right\|_{p}
$$


Summary: Consider convex optimization problem

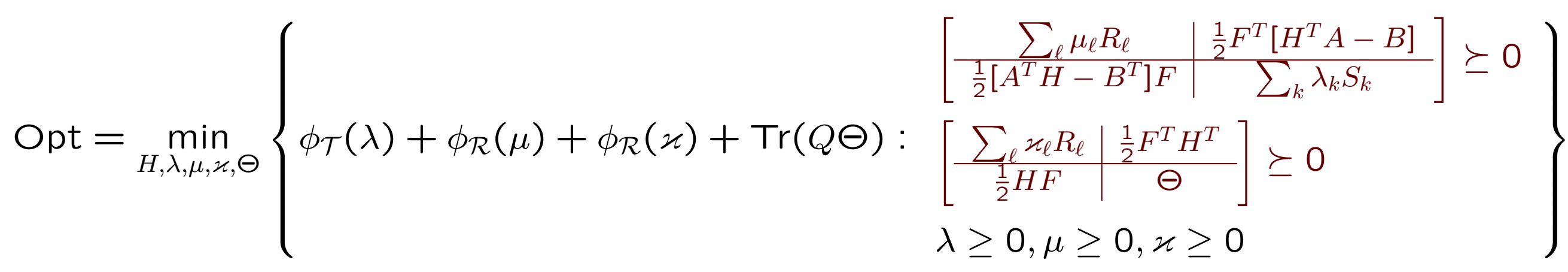

The problem is solvable, and the $H$-component $H_{*}$ of its optimal solution yields linear estimate

$$
\widehat{x}_{H_{*}}(\omega)=H_{*}^{T} \omega
$$

such that

$$
\text { Risk }_{Q,\|\cdot\|}\left[\widehat{x}_{H_{*}} \mid \mathcal{X}\right] \leq \text { Opt. }
$$

In the case of zero mean Gaussian observation noise, the estimate $\widehat{x}_{H_{*}}$ is near-optimal. 
Theorem We have

$$
\operatorname{Risk}_{Q,\|\cdot\|}\left[\widehat{x}_{H_{*}} \mid \mathcal{X}\right] \leq \text { Opt } \leq C \sqrt{\ln (2 L) \ln \left(\frac{2 M_{*}^{2} K}{\operatorname{RiskOpt}_{Q,\|\cdot\|}^{2}}\right)} \operatorname{RiskOpt}_{Q,\|\cdot\| \cdot} .
$$

Here

- $C$ is a positive absolute constant,

- RiskOpt $_{Q,\|\cdot\|}=\inf _{\widehat{x}(\cdot)}\left[\sup _{x \in \mathcal{X}} \mathbf{E}_{\eta \sim \mathcal{N}(0, Q)}\{\|B x-\widehat{x}(A x+\eta)\|\}\right]$, the infimum being taken over all estimates, is the minimax optimal $\|\cdot\|$-risk corresponding to $\mathcal{N}(0, Q)$ observation noise,

- $K, L$ are the "sizes" of ellitopes $\mathcal{X}$ and $\mathcal{B}_{*}:=F \mathcal{Y}$ :

$$
\begin{gathered}
\mathcal{X}=\left\{x: \exists t \in \mathcal{T}: x^{T} S_{k} x \leq t_{k}, k \leq K\right\}, \mathcal{Y}=\left\{y: \exists r \in \mathcal{R}: y^{T} R_{\ell} y \leq r_{\ell}, \ell \leq L\right\}, \\
-M_{*}^{2}=\max _{W}\left\{\mathbf{E}_{\eta \sim \mathcal{N}\left(0, I_{n}\right)}\left\|B W^{1 / 2} \eta\right\|^{2}: W \in \mathcal{Q}\right\}, \text { where } \\
\mathcal{Q}:=\left\{W: W \succeq 0, \exists t \in \mathcal{T}: \operatorname{Tr}\left(S_{k} W\right) \leq t_{k}, k \leq K\right\}
\end{gathered}
$$


A key fact underlying near-optimality is important by its own right:

Proposition When the unit ball $\mathcal{B}_{*}$ of the norm $\|\cdot\|_{*}$ conjugate to $\|\cdot\|$ is an ellitope:

$$
\mathcal{B}_{*}=\left\{u: \exists r \in \mathcal{R}, y: u=F y, y^{T} R_{\ell} y \leq r_{\ell}, \ell \leq L\right\}
$$

and $\eta \sim \mathcal{N}(0, Q)$, the upper bound

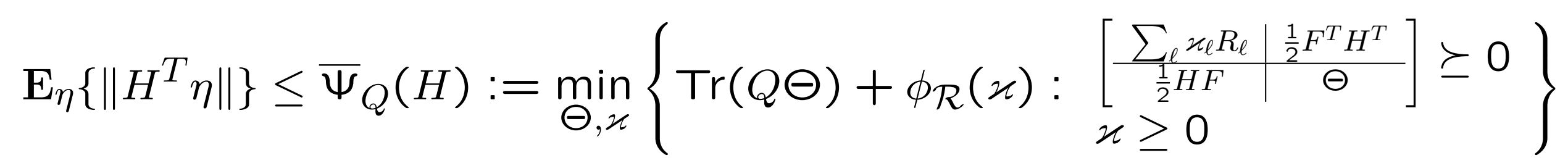

is tight:

$$
\mathbf{E}_{\eta}\left\{\left\|H^{T} \eta\right\|\right\} \leq \bar{\Psi}_{Q}(H) \leq O(1) \sqrt{\ln (2 L)} \mathbf{E}_{\eta}\left\{\left\|H^{T} \eta\right\|\right\} .
$$




\section{Variation: Recovery of partially random signals}

So far, we have considered the problem of recovering the image $B x$ of unknown deterministic signal $x$ known to belong to a given signal set $\mathcal{X}$ from noisy observations

$$
\omega=A x+\xi
$$

of linear image of the signal.

In some applications, it makes sense to consider similar problem when the signal has a random component. 
Example: Kalman Filter. Consider linear dynamical system

$$
\begin{gathered}
y_{0}=\zeta_{0} \\
y_{t+1}=P_{t} y_{t}+u_{t}+\zeta_{t},, t=1, \ldots, T \\
\omega_{t}=C_{t} y_{t}+\xi_{t} \\
\text { - } y_{t} \in \mathbb{R}^{n}: \text { states } \\
\text { - } \omega_{t} \in \mathbb{R}^{m}: \text { observations } \quad: u_{t}: \text { controls } \\
\xi_{t}: \text { random observation noise }
\end{gathered}
$$

We want is to recover from observations $\omega_{1}, \ldots, \omega_{T}$ linear image

$$
z:=R\left[y_{0} ; \ldots ; y_{T+1}\right]
$$

of the state trajectory, e.g., $y_{T}$ ("filtering") or $y_{T+1}$ ("forecast"), etc

Note: In the classical Kalman Filter,

- $\zeta_{0}, \ldots, \zeta_{T}$ are independent of each other zero mean Gaussian

- $\xi_{1}, \ldots, \xi_{T}$ are independent of each other and of $\zeta_{t}$ 's zero mean Gaussian

- $u_{1}, \ldots, u_{T}$ are deterministic and known (reduces to the case when $u_{t} \equiv 0$ ) 
- When modeling the situation as an estimation problem, we can use state equation to express the states $y_{t}$ as known linear functions of controls $u_{t}$ and process noises $\zeta_{t}$, thus arriving at the model

$$
\begin{gathered}
\omega=A[u ; \zeta]+\xi, \quad z=B[u ; \zeta] \\
{\left[u=\left[u_{1} ; \ldots ; u_{T}\right], \zeta=\left[\zeta_{0} ; \ldots ; \zeta_{T}\right], \xi=\left[\xi_{1} ; \ldots ; \xi_{T}\right]\right]}
\end{gathered}
$$

- When quantifying the performance of a candidate estimate $\widehat{x}(\omega)$, one can consider risk of the form

$$
\operatorname{Risk}[\widehat{x}]=\sup _{u} \mathbf{E}_{\xi, \zeta}\{\|B[u ; \zeta]-\widehat{x}(A[u ; \zeta]+\xi)\|\} .
$$


Situation: We observe noisy linear image

$$
\omega=A[u ; \zeta]+\xi=A_{d} u+A_{s} \zeta+\xi
$$

of "signal" $x=[u ; \zeta]$ with deterministic component $u$ and stochastic component $\zeta$. We assume that

- $u$ is "uncertain-but-bounded" - is known to belong to a given set $\mathcal{U}$

- $\zeta$ and $\xi$ have partially known distributions. Specifically, for given $Q_{\zeta} \succ 0, Q_{\xi} \succ 0$ it holds

$$
\mathrm{V}[\xi]=\mathbf{E}\left\{\xi \xi^{T}\right\} \preceq Q_{\xi}, \quad \mathrm{V}[\zeta]=\mathbf{E}\left\{\zeta \zeta^{T}\right\} \preceq Q_{\zeta}
$$

Given matrix $B=\left[B_{d}, B_{s}\right]$ and a norm $\|\cdot\|$ on the image space of $B$, we want to recover $B[u ; \zeta]=B_{d} u+B_{s} \zeta$, quantifying the recovery error in $\|\cdot\|$. The performance of a candidate estimate $\widehat{x}(\cdot)$ is quantified by

$$
\operatorname{Risk}[\widehat{x}]=\sup _{u \in \mathcal{U}} \sup _{P \in \mathcal{P}} \mathbf{E}_{[\xi ; \zeta] \sim P}\{\|B[u ; \zeta]-\widehat{x}(A[u ; \zeta]+\xi)\|\}
$$

$\left[\mathcal{P}\right.$ : probability distributions such that $\left.\mathbf{E}_{[\xi ; \zeta] \sim P}\left\{\xi \xi^{T}\right\} \preceq Q_{\xi}, \mathbf{E}_{[\xi ; \zeta] \sim P}\left\{\zeta \zeta^{T}\right\} \preceq Q_{\zeta}\right]$

Goal: Build "presumably good" linear estimate $\widehat{x}_{H}(\omega)=H^{T} \omega$. 
Assumption: $\mathcal{U}$ is a basic ellitope, and the unit ball of the norm $\|\cdot\|_{*}$ dual to $\|\cdot\|$ is an ellitope:

$$
\begin{aligned}
\mathcal{U} & =\left\{u: \exists t \in \mathcal{T}: u^{T} S_{k} u \leq t_{k}, k \leq K\right\} \\
\left\{v:\|v\|_{*} \leq 1\right\} & =\left\{v: \exists r \in \mathcal{R}, w: v=M w, w^{T} R_{\ell} w \leq r_{\ell}, \ell \leq L\right\}
\end{aligned}
$$

- For a candidate linear estimate $\widehat{x}_{H}(\omega)=H^{T} \omega, u \in \mathcal{U}$, and a distribution $P$ of $[\xi ; \zeta]$ satisfying the bounds on the matrices of second moments of $\xi$ and $\zeta$ we have

$$
\begin{aligned}
\mathbf{E}_{[\xi, \zeta] \sim P} & \left\{\left\|B_{d} u+B_{s} \zeta-H^{T}\left[A_{d} u+A_{s} \zeta+\xi\right]\right\|\right\} \\
& \left.\leq \| B_{d}-H^{T} A_{d}\right] u \|+\mathbf{E}_{[\xi ; \zeta] \sim P}\left\{\left\|H^{T} \xi\right\|\right\}+\mathbf{E}_{[\xi ; \zeta] \sim P}\left\{\left\|\left[B_{s}-H^{T} A_{s}\right] \zeta\right\|\right\}
\end{aligned}
$$

As we know,

$$
\begin{aligned}
& u \in \mathcal{U} \Rightarrow\left\|\left[B_{d}-H^{T} A_{d}\right] u\right\| \leq \min _{\lambda \geq 0, \nu \geq 0}\left\{\phi_{\mathcal{T}}(\lambda)+\phi_{\mathcal{R}}(\nu):\left[\begin{array}{c|c}
\sum_{\ell} \nu_{\ell} R_{\ell} & \frac{1}{2} M^{T}\left[B_{d}-H^{T} A_{d}^{T}\right] \\
\hline \frac{1}{2}\left[B_{d}^{T}-A_{d}^{T} H\right] & \sum_{k} \lambda_{k} S_{k}
\end{array}\right] \succeq 0\right\} \\
& \vee[\xi] \preceq Q_{\xi} \Rightarrow \mathbf{E}_{\xi}\left\{\left\|H^{T} \xi\right\|\right\} \leq \min _{\mu \geq 0, G}\left\{\operatorname{Tr}\left(G Q_{\xi}\right)+\phi_{\mathcal{R}}(\mu):\left[\begin{array}{c|c}
G & \frac{1}{2} H M \\
\hline \frac{1}{2} M^{T} H^{T} & \sum_{\ell} \mu_{\ell} R_{\ell}
\end{array}\right] \succeq 0\right\} \\
& \vee[\zeta] \preceq Q_{\zeta} \Rightarrow \mathbf{E}_{\zeta}\left\{\left\|\left[B_{s}-H^{T} A_{s}\right] \zeta\right\|\right\} \leq \min _{\mu \geq 0, G}\left\{\begin{array}{c}
\operatorname{Tr}\left(G Q_{\xi}\right)+\phi_{\mathcal{R}}(\mu): \\
{\left[\begin{array}{c|c}
G & \frac{1}{2}\left[B_{s}^{T}-A_{s}^{T} H\right] M \\
\hline \frac{1}{2} M^{T}\left[B_{s}-H^{T} A_{s}\right] & \sum_{\ell} \mu_{\ell} R_{\ell}
\end{array}\right] \succeq 0}
\end{array}\right\}
\end{aligned}
$$


Bottom line: In the situation, consider the convex optimization problem

$$
\begin{array}{r}
\text { Opt }=\min _{\substack{H, \lambda, \nu, \mu, \mu^{\prime}, G, G^{\prime}}}\left\{\phi_{\mathcal{T}}(\lambda)+\phi_{\mathcal{R}}(\nu)+\phi_{\mathcal{R}}(\mu)+\phi_{\mathcal{R}}\left(\mu^{\prime}\right)+\operatorname{Tr}\left(Q_{\xi} G\right)+\operatorname{Tr}\left(Q_{\zeta} G^{\prime}\right):\right. \\
\lambda \geq 0, \nu \geq 0, \mu \geq 0, \mu^{\prime} \geq 0,\left[\begin{array}{c|c}
\sum_{\ell} \nu_{\ell} R_{\ell} & \frac{1}{2} M^{T}\left[B_{d}-H^{T} A_{d}^{T}\right] \\
\frac{1}{2}\left[B_{d}^{T}-A_{d}^{T} H\right] & \sum_{k} \lambda_{k} S_{k} \\
\left.\begin{array}{c|c|c}
\frac{1}{2}\left[B_{s}^{T}-A_{s}^{T} H\right] M \\
\frac{1}{2} M^{T}\left[B_{s}-H^{T} A_{s}\right] & \sum_{\ell} \mu_{\ell}^{\prime} R_{\ell}
\end{array}\right] \succeq 0
\end{array}\right\} \\
\quad\left[\begin{array}{c|c}
\frac{1}{2} M^{T} H^{T} & \sum_{\ell} \mu_{\ell} R_{\ell}
\end{array}\right] \succeq 0
\end{array}
$$

The problem is efficiently solvable, and the $H$-component $H_{*}$ of its optimal solution gives rise to linear estimate $\widehat{x}_{H_{*}}(\Omega)=H_{*}^{T} \omega$ such that

$$
\operatorname{Risk}\left[\widehat{x}_{H_{*}}\right] \leq \text { Opt. }
$$




\section{How it works}

System: Discretized pendulum $\ddot{x}=-\dot{x}-\kappa x$ :

$$
\begin{aligned}
{\left[\begin{array}{l}
x_{t+1} \\
v_{t+1}
\end{array}\right]=} & {\left[\begin{array}{cc}
0.9990 & 0.0951 \\
-0.0190 & 0.9039
\end{array}\right]\left[\begin{array}{l}
x_{t} \\
v_{t}
\end{array}\right]+\left(u_{t}+\zeta_{t}\right)\left[\begin{array}{l}
0.0048 \\
0.0951
\end{array}\right], 1 \leq t \leq 128 } \\
& x_{t} \\
& {\left[\left[\begin{array}{l}
x_{1} \\
v_{1}
\end{array}\right] \sim \mathcal{N}(0, I), \zeta_{t} \sim \mathcal{N}\left(0,0.05^{2}\right), \xi \sim \mathcal{N}\left(0,0.05^{2}\right),\left|u_{t}\right| \leq 0.1\right] }
\end{aligned}
$$

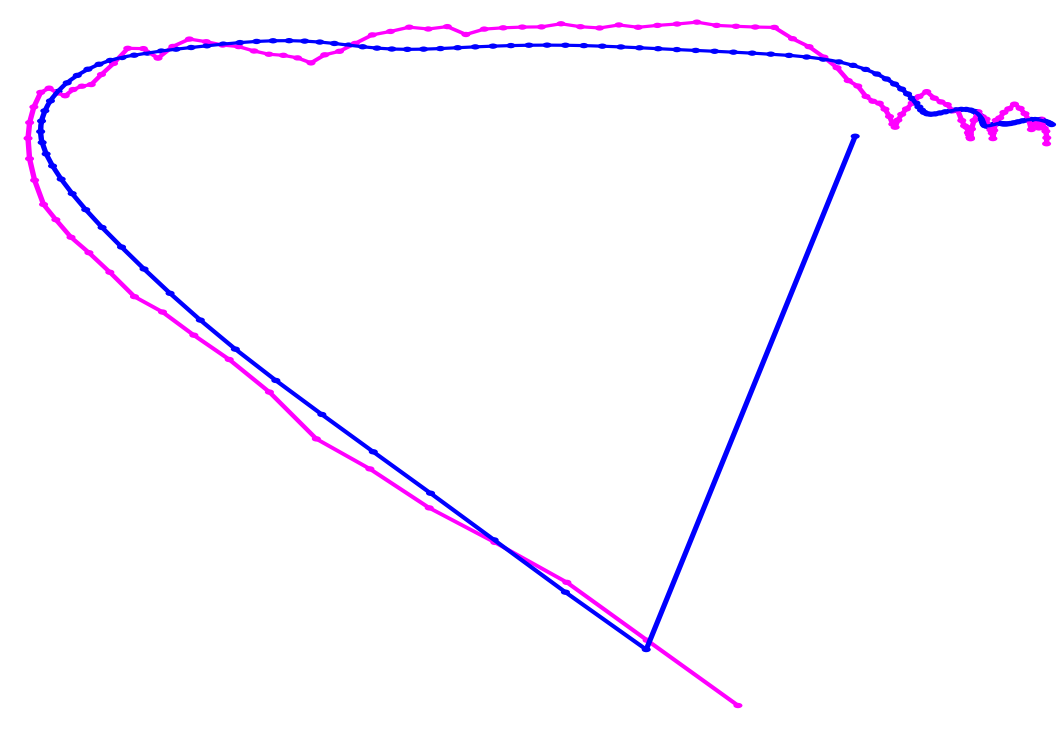

sample trajectory and forecasts

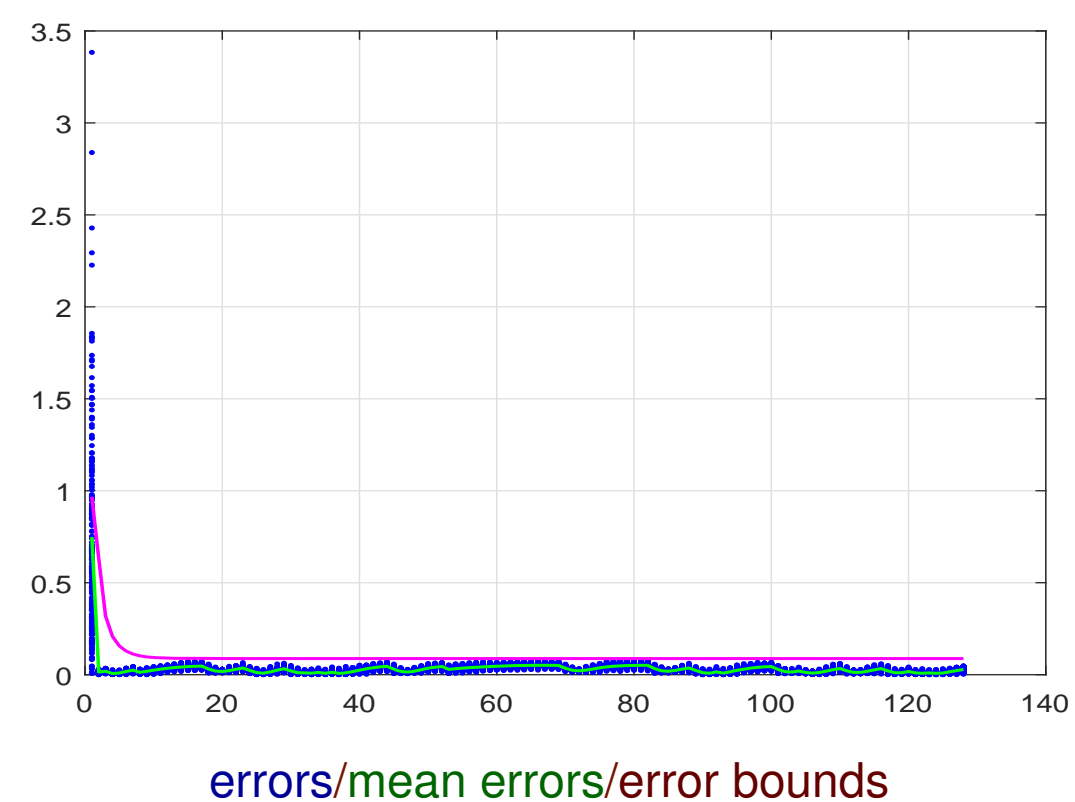




\section{Recovery under uncertain-but-bounded noise}

So far, we have considered recovering $B x, x \in \mathcal{X}$, from observation

$$
\omega=A x+\eta
$$

affected by random noise $\eta$. Let us now assume that $\eta$ is "uncertain-butbounded:" all we know is that

$$
\eta \in \mathcal{N}
$$

with a given convex and compact set $\mathcal{H}$.

Risk: In the case in question, natural definition of risk of a candidate estimate $\widehat{x}(\cdot)$ is

$$
\operatorname{Risk}_{\mathcal{H},\|\cdot\|}[\widehat{x}(\cdot) \mid \mathcal{X}]=\sup _{x \in \mathcal{X}, \eta \in \mathcal{H}}\|B x-\widehat{x}(A x+\eta)\| .
$$


Observation: Signal recovery under uncertain-but-bounded noise reduces to the situation where there is no observation noise at all.

Indeed, let us treat as the signal underlying observation the pair $z=[x ; \eta] \in \mathcal{Z}:=\mathcal{X} \times \mathcal{H}$ and replace $A$ with $\bar{A}=[A, I]$ and $B$ with $\bar{B}=[B, 0]$, so that

$$
\omega=\bar{A}[x ; \eta] \quad \& \quad B x=\bar{B}[x ; \eta],
$$

thus reducing signal recovery to recovering $\bar{B} z, z \in \mathcal{Z}$, from noiseless observation $\bar{A} z$.

From now on, let us focus on the problem of recovering the image $B x \in \mathbb{R}^{\nu}$ of unknown signal $x \in \mathbb{R}^{n}$ known to belong to signal set $\mathcal{X} \subset \mathbb{R}^{n}$ via observation

$$
\omega=A x \in \mathbb{R}^{m} \text {. }
$$

Given norm $\|\cdot\|$ on $\mathbb{R}^{\nu}$, we quantify the performance of an estimate $\widehat{x}(\cdot)$ : $\mathbb{R}^{m} \rightarrow \mathbb{R}^{\nu}$ by its $\|\cdot\|$-risk

$$
\operatorname{Risk}_{\|\cdot\|}[\widehat{x} \mid \mathcal{X}]=\sup _{x \in \mathcal{X}}\|B x-\widehat{x}(A x)\|
$$


Observation: Assuming that $\mathcal{X}$ is computationally tractable convex compact set and $\|\cdot\|$ is computationally tractable, it is easy to build an efficiently computable optimal within factor 2 nonlinear estimate:

Given $\omega$, let us solve the convex feasibility problem

$$
\text { Find } y \in \mathcal{Y}[\omega]:=\{y \in \mathcal{X}: A y=\omega\} \text {. }
$$

and take, as $\widehat{x}(\omega)$, the vector By, where $y$ is (any) solution to the feasibility problem.

Note: When $\omega$ stems from a signal $x \in \mathcal{X}$, the set $\mathcal{Y}[\omega]$ contains $x$

$\Rightarrow \widehat{x}(\cdot)$ is well defined 


$$
x \in \mathcal{X}, \omega=A x \Rightarrow \widehat{x}(\omega)=B y \quad[y \in \mathcal{Y}[\omega]=\{y \in \mathcal{X}: A y=\omega\}]
$$

Performance analysis: Denote

$$
\begin{aligned}
\mathfrak{R} & =\max _{y, z}\left\{\frac{1}{2}\|B[y-z]\|: y, z \in \mathcal{X}, A[y-z]=0\right\} \\
& =\frac{1}{2}\left\|B\left[y_{*}-z_{*}\right]\right\| \quad\left[y_{*}, z_{*} \in \mathcal{X}, A\left[y_{*}-z_{*}\right]=0\right]
\end{aligned}
$$

Claim A: For every estimate $\widetilde{x}(\cdot)$ it holds $\operatorname{Risk}_{\|\cdot\|}[\widetilde{x} \mid \mathcal{X}] \geq \Re$.

Indeed, the observation $\omega=A y_{*}=A z_{*}$ stems from both $y_{*}$ and $z_{*}$, whence the $\|\cdot\|$-risk of every estimate is at least $\frac{1}{2}\left\|B y_{*}-B z_{*}\right\|=\Re$.

Claim B: One has Risk $\|_{\|\cdot\|}[\widehat{x} \mid \mathcal{X}] \leq 2 \Re$.

Indeed, let $\omega=A x$ with $x \in \mathcal{X}$, and let $\widehat{x}(\omega)=B \widehat{y}$ with $\widehat{y} \in \mathcal{Y}[\omega]$. Then both $x, \widehat{y}$ belong to $\mathcal{Y}[\omega]$

$$
\Rightarrow \frac{1}{2}\|B[x-\widehat{y}]\| \leq \Re .
$$


Question: We have built optimal, within factor 2, estimate. How to upperbound its $\|\cdot\|$-risk?

Let $\mathcal{X}$ and the unit ball $\mathcal{B}_{*}$ of the norm $\|\cdot\|_{*}$ conjugate to $\|\cdot\|$ be ellitopes:

$$
\begin{aligned}
\mathcal{X} & =\left\{x=P y: y \in \mathcal{Y}:=\left\{y: \exists t \in \mathcal{T}: y^{T} S_{k} y \leq t_{k}, k \leq K\right\}\right\} \\
\mathcal{B}_{*} & =\left\{u=Q v: v \in \mathcal{V}:=\left\{v: \exists r \in \mathcal{R}: v^{T} R_{\ell} v \leq r_{\ell}, \ell \leq L\right\}\right\}
\end{aligned}
$$

Then the $\|\cdot\|$-risk of the optimal, within factor 2 , efficiently computable nonlinear estimate $\widehat{x}(\cdot)$ can be tightly lower- and upper-bounded as follows.

- Assume that $\operatorname{Ker} A \cap \mathcal{X} \neq\{0\}$ (otherwise the risk is zero), the set $\mathcal{X}_{A}=\{x \in \mathcal{X}: A x=0\}$ is an ellitope:

$$
\mathcal{X}_{A}=\left\{x=F w, w \in \mathcal{W}:=\left\{w: \exists t \in \mathcal{T}: w^{T} T_{k} w \leq t_{k}, k \leq K\right\}\right\}
$$

Indeed, setting $E=\{y: A P y=0\}$, the set

$$
\mathcal{Y}_{A}=\left\{y \in E: \exists t \in \mathcal{T}: y^{T} S_{k} y \leq t_{k}, k \leq K\right\}
$$

is a basic ellitope in some $\mathbb{R}^{N^{\prime}} \Rightarrow \mathcal{X}_{A}=\left\{P y: y \in \mathcal{Y}_{A}\right\}$ is an ellitope. 
- Consequently,

$$
\begin{aligned}
\mathfrak{R} & :=\max _{x^{\prime}, x^{\prime \prime} \in \mathcal{X}}\left\{\frac{1}{2}\left\|B\left[x^{\prime}-x^{\prime \prime}\right]\right\|: A\left[x^{\prime}-x^{\prime \prime}\right]=0\right\} \\
& =\max _{x \in \mathcal{X} A}\|B x\|=\max _{w \in \mathcal{W}}\|B F w\| \\
& =\|B F\|_{\|\cdot\|_{w} \rightarrow\|\cdot\|} \quad\left[\|\cdot\|_{w} \text { : norm with the unit ball } \mathcal{W}\right],
\end{aligned}
$$

and

$$
\mathfrak{R} \leq \text { Opt } \leq 4 \ln (5[K+L]) \Re
$$

where the efficiently computable quantity Opt is given by

$$
\text { Opt }=\min _{\lambda, \mu}\left\{\phi_{\mathcal{T}}(\lambda)+\phi_{\mathcal{R}}(\mu): \begin{array}{l}
\lambda \geq 0, \mu \geq 0 \\
{\left[\begin{array}{ll|l}
\lambda \mu_{\ell} R_{\ell} & \frac{1}{2} Q^{T} B F \\
\hline \frac{1}{2} F^{T} B^{T} Q & \sum_{k} \lambda_{k} T_{k}
\end{array}\right] \succeq 0}
\end{array}\right\} .
$$

$\Rightarrow$ The optimal $\|\cdot\|$-risk is $\geq \Re \geq \frac{\text { Opt }}{4 \ln (5[K+L])}$, and

$$
\operatorname{Risk}_{\|\cdot\|}[\widehat{x} \mid \mathcal{X}] \leq 2 \Re \leq 2 \text { Opt. }
$$


In fact, under mild assumptions a linear estimate is near-optimal:

Theorem Consider the problem of recovering $B x$ in $\|\cdot\|, x \in \mathcal{X}$, via observation $\omega=A x$. Let the signal set $\mathcal{X}$ be an ellitope, and the unit ball $\mathcal{B}_{*}$ of the norm conjugate to $\|\cdot\|$ be a basic ellitope:

$$
\begin{gathered}
\mathcal{X}=\left\{x=P y: y \in \mathcal{Y}:=\left\{y: \exists t \in \mathcal{T}: y^{T} S_{k} y \leq t_{k}, k \leq K\right\}\right\}, \\
\mathcal{B}_{*}=\left\{u: \exists r \in \mathcal{R}: u^{T} R_{\ell} u \leq r_{\ell}, \ell \leq L\right\}
\end{gathered}
$$

Then the linear estimate $\widehat{x}(\omega)=H_{*}^{T} \omega$ yielded by the $H$-component of optimal solution to the efficiently solvable optimization problem

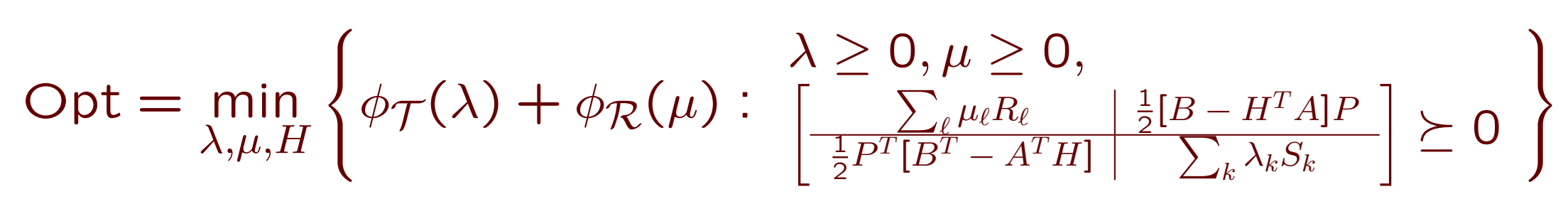

is near-optimal:

$$
\operatorname{Risk}_{\|\cdot\|}\left[\widehat{x}_{H_{*}} \mid \mathcal{X}\right] \leq \text { Opt } \leq 4 \ln (5[K+L]) \operatorname{RiskOpt}_{\|\cdot\|}[\mathcal{X}],
$$

where RiskOpt $\|_{\|\cdot\|}[\mathcal{X}]=\inf _{\widehat{x}(\cdot)} \operatorname{Risk}_{\|\cdot\|}[\widehat{x} \mid \mathcal{X}]$. is the minimax risk. 
Sketch of the proof:

A. The $\|\cdot\|$-risk of a linear estimate $\widehat{x}_{H}(\omega)=H^{T} \omega$ is

$$
\operatorname{Risk}_{\|\cdot\|}\left[\widehat{x}_{H} \mid \mathcal{X}\right]=\max _{x \in \mathcal{X}}\left\|\left[B-H^{T} A\right] x\right\|=\max _{y \in \mathcal{Y}}\left\|\left[B-H^{T} A\right] P y\right\|
$$

which is nothing but $\left\|\left[B-H^{T} A\right] P\right\|_{\|\cdot\|_{y} \rightarrow\|\cdot\|}$, where $\|\cdot\|_{y}$ is the norm with the unit ball $\mathcal{Y}$. By the theorem on upper-bounding operator norms, we have

$$
\begin{gathered}
\left.\operatorname{Risk}_{\|\cdot\|}\left[\widehat{x}_{H} \mid \mathcal{X}\right] \leq \min _{\lambda, \mu}\left\{\phi_{\mathcal{T}}(\lambda)+\phi_{\mathcal{R}}(\mu): \begin{array}{l|l}
\lambda \geq 0, \mu \geq 0 \\
{\left[\begin{array}{l}
\sum_{\ell} \mu_{\ell} R_{\ell} \\
{\left[\frac{1}{2} P^{T}\left[B^{T}-A^{T} H\right]\right.}
\end{array}\right.} & \frac{1}{2}\left[B-H^{T} A\right] P \\
\sum_{k} \lambda_{k} S_{k}
\end{array}\right] \succeq 0\right\} \\
\operatorname{Risk}_{\|\cdot\|}\left[\widehat{x}_{H_{*}} \mid \mathcal{X}\right] \leq \text { Opt. }
\end{gathered}
$$

B. As we have seen, the quantity

$$
\mathfrak{R}=\max _{x}\{\|B x\|: A x=0, x \in \mathcal{X}\}
$$

is a lower bound on the minimal optimal $\|\cdot\|$-risk $\operatorname{Risk}_{\|\cdot\|}^{*}[\mathcal{X}]$, and $\mathfrak{R}$ can be tightly (within factor $4 \ln (5[K+L]))$ upper-bounded by the optimal value in an explicit conic problem. On the closest inspection, utilizing conic duality, the optimal value in question turns out to be equal to Opt

$\Rightarrow$ The (upper bound on the) $\|\cdot\|$-risk of the linear estimate $\widehat{x}_{H_{*}}$ is within the factor $4 \ln (5[K+$ $L]$ ) of the lower bound $\mathfrak{R}$ on the minimax optimal $\|\cdot\|$-risk. 